\title{
Low Complexity Delay and Phase-Locked Loops
}

by

\author{
Gordon Allan
}

\author{
A Thesis Submitted to the \\ Faculty of Graduate Studies and Research \\ in Fulfillment of the Requirements for the Degree of
}

\section{Doctor of Philosophy}

Ottawa-Carleton Institute for Electrical Engineering

Department of Electronics

Carleton University

(c)Gordon Allan 2009 


$\begin{array}{ll}\begin{array}{l}\text { Library and Archives } \\ \text { Canada }\end{array} & \begin{array}{l}\text { Bibliothèque et } \\ \text { Archives Canada }\end{array} \\ \begin{array}{l}\text { Published Heritage } \\ \text { Branch }\end{array} & \begin{array}{l}\text { Direction du } \\ \text { Patrimoine de l'édition }\end{array} \\ \begin{array}{l}\text { 395 Wellington Street } \\ \text { Ottawa ON K1A 0N4 } \\ \text { Canada }\end{array} & \begin{array}{l}\text { 395, rue Wellington } \\ \text { Ottawa ON K1A ON4 } \\ \text { Canada }\end{array}\end{array}$

Your file Votre reference

ISBN: 978-0-494-60098-6

Our file Notro référence

ISBN: 978-0-494-60098-6

NOTICE:

AVIS:

The author has granted a nonexclusive license allowing Library and Archives Canada to reproduce, publish, archive, preserve, conserve, communicate to the public by telecommunication or on the Internet, loan, distribute and sell theses worldwide, for commercial or noncommercial purposes, in microform, paper, electronic and/or any other formats.

The author retains copyright ownership and moral rights in this thesis. Neither the thesis nor substantial extracts from it may be printed or otherwise reproduced without the author's permission.

L'auteur a accordé une licence non exclusive permettant à la Bibliothèque et Archives Canada de reproduire, publier, archiver, sauvegarder, conserver, transmettre au public par télécommunication ou par l'Internet, prêter, distribuer et vendre des thèses partout dans le monde, à des fins commerciales ou autres, sur support microforme, papier, électronique etlou autres formats.

L'auteur conserve la propriété du droit d'auteur et des droits moraux qui protège cette thèse. $\mathrm{Ni}$ la thèse ni des extraits substantiels de celle-ci ne doivent être imprimés ou autrement reproduits sans son autorisation.
In compliance with the Canadian Privacy Act some supporting forms may have been removed from this thesis.

While these forms may be included in the document page count, their removal does not represent any loss of content from the thesis.
Conformément à la loi canadienne sur la protection de la vie privée, quelques formulaires secondaires ont été enlevés de cette thèse.

Bien que ces formulaires aient inclus dans la pagination, il n'y aura aucun contenu manquant.

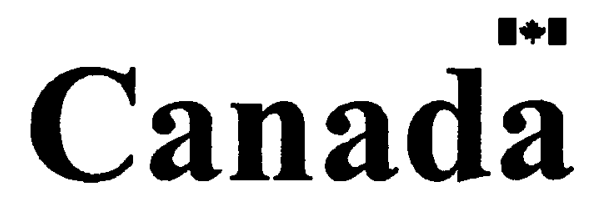




\section{Abstract}

Currently, delay-locked loops (DLLs) and phase-locked loops (PLLs) are too large and inefficient for extensive use as clock-alignment circuits in complex ICs. Their area tends to be dominated by the loop-filter, which requires large capacitors that scale proportionally with the loop-gain. With advances in technology, supply swings are reduced and the sensitivity of the loop must increase, requiring larger filters that often make fully integrated solutions impractical.

In this work a new cascaded charge-pump (CCP) and dynamically rotated filter structure are introduced to replace the conventional charge-pump. The cascaded charge-pump can be formed with digital tri-state buffers, but connected in such a way that they act as a network of small and simple analog charge-pumps. The structure generates a thermometer-coded vector of analog control voltages to modulate a voltage-controlled oscillator ( $\mathrm{VCO}$ ) or delay-line ( $\mathrm{VCDL})$. By implementing the VCO control with a vector, rather than a single voltage, the VCO gain/node $\left(K_{V}\right)$ can be arbitrarily reduced. The reduction in $K_{V}$ creates a corresponding reduction in capacitive requirements, making the circuits far more area efficient.

Using this structure a very compact low-power PLL was implemented in $0.18 \mu \mathrm{m}$ CMOS for clock management and distribution. Like digital PLLs, it is composed of standard-cells, can be mixed with regular logic, and is digitally placed \& routed, but unlike digital PLLs it does not suffer from quantization jitter. Its area is only $0.008 \mathrm{~mm}^{2}$ (650 'gates') when configured as a x32 clock multiplier with a $200 \mathrm{kHz}$ loop-BW. In this configuration, it consumes only $190 \mu W @ 128 M h z$. It can perform efficient clock distribution, cleansing a noisy low-frequency reference and synchronizing outputs with cycle-to-cycle jitter below $5.6 p s_{r m s}$. With a lock-range between $60-172 \mathrm{MHz}$, adjustable loop dynamics and last lock frequency memory, it is less than $1 / 5^{\text {th }}$ the size and $1 / 15^{\text {th }}$ the power of previous PLLs at similar frequencies and noise levels. 


\section{Acknowledgments}

I would like to thank my family for tolerating the many long hours required to support both full-time work and academics. Without their support and encouragement this work would not have been possible. No less important was the constant advice, insight and encouragement of my advisor, Dr. John Knight.

I appreciate the time of a number of others, including the examining committee, who have been available to provide technical support, bounce ideas back-and-forth, and critique not only this work but other ideas during the course of the research. While certainly not exhaustive, this list includes Calvin Plett, Reza Yousefi, Tom Riley, Norm Filiol, Bill Bereza, John Rogers and Nagui Mikhail.

For their support of my academic endeavors, and for the continuing education received in industry, I'd like to thank my employers over the course of the work, particularly Hittite Microwave Corporation. I'd like to thank my colleagues Mark Cloutier, Shawn Sellars, Tolga Pamir and Tudor Lipan for constantly broadening my scope of knowledge, and Kashif Sheikh for providing some of the software used to generate plots in the thesis.

Finally, I would like to thank those individuals and organizations which were instrumental in funding the early phases of the research. Without their support it would have been impossible to devote my time to the work. This includes Carleton's Department of Electronics, NSERC, Kaben Research, Micronet, and CITO-OCE. 


\section{Contents}

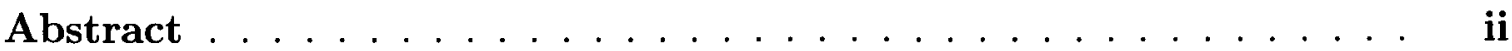

Acknowledgments $\ldots \ldots \ldots \ldots \ldots \ldots$ iii

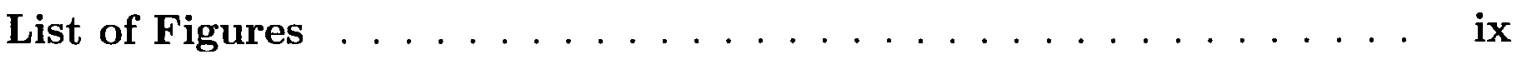

List of Tables $\ldots \ldots \ldots \ldots \ldots$ xii

Abbreviations, Definitions, Symbols and Acronyms . . . . . . . xiii

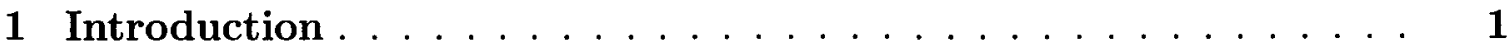

1.1 Applications of Phase and Delay Locked Loops . . . . . . . . . . . . . 1

1.1.1 Synthesizers for wireless communications - Low Noise . . . . . 1

1.1.2 Synthesizers for wired communications - High Density . . . . . 2

1.2 Goal: Small, Low Power Synthesizers . . . . . . . . . . . . . 2

1.2.1 The Figure of Merit . . . . . . . . . . . . . . . 3

1.3 Theme of Thesis: The Cascaded Charge-Pump (CCP) . . . . . . . . 3

1.3.1 Drastically Reduced Size . . . . . . . . . . . . . . . . . 3

1.3.2 Improved Noise Suppression . . . . . . . . . . . . . . . 4

1.3.3 Other improvements... . . . . . . . . . . . . . . 4

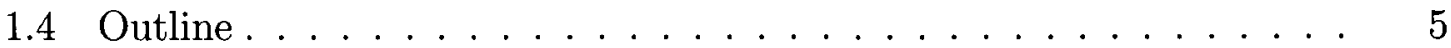

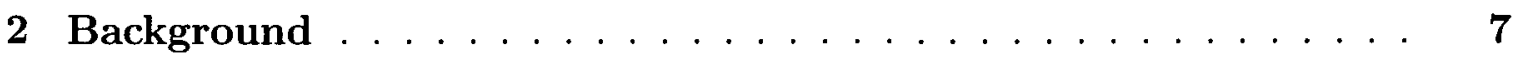

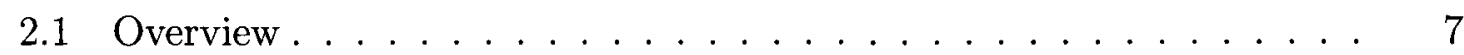

2.2 Basic PLL and DLL Operation $\ldots \ldots \ldots \ldots \ldots$

2.3 DLLs vs PLLs . . . . . . . . . . . . . . . . . 9

2.3 .1 Reference Noise . . . . . . . . . . . . . . . . . . . 9

2.3 .2 Delay-Line Noise . . . . . . . . . . . . . . . . . . . . . 10

2.3 .3 Clock Multiplication . . . . . . . . . . . . . . . 10 
2.3.4 Clock Alignment . . . . . . . . . . . . . . . . . 11

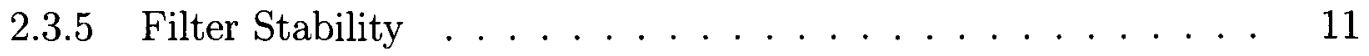

2.3.6 Comparison of Applications: DLL vs PLL . . . . . . . . . 12

2.4 Loop Theory . . . . . . . . . . . . . . . . . . . . . . . 12

2.4.1 PLL Open-loop Transfer Function . . . . . . . . . . . . . 13

2.4 .2 Closing the Loop . . . . . . . . . . . . . . . . . . 17

2.5 Effect of Loop gain on Filter size . . . . . . . . . . . . . . 19

2.6 Noise Sources and Transfer Characteristics . . . . . . . . . . . . . . 19

2.6.1 Optimal Loop Bandwidth . . . . . . . . . . . . . . . 21

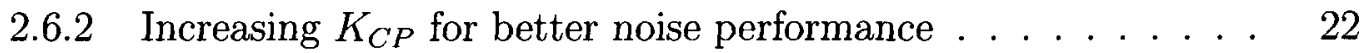

2.7 Architectural Overview . . . . . . . . . . . . . . . . . . 23

2.7.1 Analog, Digital or Mixed-Signal . . . . . . . . . . . 23

2.7.2 Analog Implementation Challenges . . . . . . . . . . . 24

2.7 .3 Digital Implementation Overview . . . . . . . . . . . . . 27

2.7.4 Digital Implementation Challenges . . . . . . . . . . . . . . 29

2.7 .5 Mixed-Signal PLLs/DLLs . . . . . . . . . . . . . . . . . 32

2.8 Literature Search . . . . . . . . . . . . . . . . . . . . . . . 33

2.8.1 Analog Implementations . . . . . . . . . . . . . . . . . 33

2.8 .2 Digital Architectures . . . . . . . . . . . . . . . . . . 35

2.8.3 Mixed-Signal Architectures . . . . . . . . . . . . . . . . . . 38

3 Cascaded Charge-Pump: A System Level Perspective . . . . . . . 41

3.1 Overview . . . . . . . . . . . . . . . . . . . . . . . . . 41

3.2 Cascaded Charge-Pump Simplified . . . . . . . . . . . . . . . 42

3.3 Current Steering for Vectored Control . . . . . . . . . . . . . . . . . 44

3.3.1 Current-Steering in the Cascaded Charge Pump . . . . . . . 44

3.3.2 Transition between control nodes . . . . . . . . . . . 47

3.3.3 Example of Locking a DLL with a Cascaded Charge-Pump . . 48

3.3.4 Use in PLLs vs DLLs . . . . . . . . . . . . . . . . . . . 50

3.4 Conventional vs a Cascaded Charge-Pump Controlled PLL . . . . . . 51

3.4.1 Effect of non-linear current on Acquisition . . . . . . . . . 54

3.5 Benefits of Reduced VCO Gain . . . . . . . . . . . . . . . . . 55

3.5.1 Improved Noise Suppression . . . . . . . . . . . . . . 55 
3.6 System Level PLL Simulator . . . . . . . . . . . . . . . . . . 56

3.7 Simulation of Noise sensitivity vs $K_{V} \ldots \ldots \ldots \ldots \ldots$

3.7.1 Capacitance Reduction Benefits . . . . . . . . . . . . 62

3.8 Using the Architecture in DLLs instead of PLLs . . . . . . . . . . 63

3.9 Summary . . . . . . . . . . . . . . . . . . 63

4 Circuit Implementation . . . . . . . . . . . . . . . . . . 68

4.1 Overview . . . . . . . . . . . . . . . . . . 68

4.2 Simplifying the Cascaded Charge-Pump Hardware . . . . . . . . . . . 69

4.2.1 Inverting Thermometer Codes . . . . . . . . . . . . 70

4.2.2 Removing redundant transistors and halving the circuit size . 72

4.3 VCO Modulation . . . . . . . . . . . . . . . . . . . . . 73

4.4 Gain, Source Impedance and Consistency . . . . . . . . . . . . . 74

4.4.1 Finite Current-Source Impedance . . . . . . . . . . . . . 74

4.4.2 Characterizing Charge-Pump Gain . . . . . . . . . . 78

4.5 Filter Stages . . . . . . . . . . . . . . . . . . . . . 83

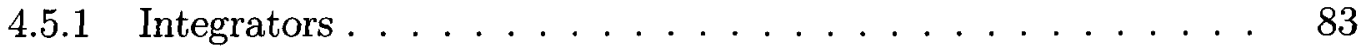

$4.5 .2 \quad$ Moving $\omega_{p 1}>0 \ldots \ldots \ldots \ldots \ldots$

4.5.3 Implementing a stabilizing zero $\omega_{z}$ - Type II PLLs . . . . . . . 84

4.6 Sharing Filter Sections _ . . . . . . . . . . . . . . . 85

4.6.1 Effective Capacitance Multiplication _. . . . . . . . 88

4.7 Stabilizing the Digital Values . . . . . . . . . . . . . . . . 89

4.8 Leakage Sensitivity . . . . . . . . . . . . . . . . . . . . 90

4.8.1 Charge-Pump Leakage . . . . . . . . . . . . . . . . . . 91

4.8.2 Reduced Effects of Dielectric Leakage . . . . . . . . . . . . 91

4.9 Supply Noise Sensitivity _ . . . . . . . . . . . . . . . . . . 92

4.9 .1 Varactor Sensitivity . . . . . . . . . . . . . . . . 92

4.9 .2 Switch Sensitivity . . . . . . . . . . . . . . . . . 92

4.9 .3 Supply Filtering . . . . . . . . . . . . . . . . . . . . 93

4.10 Phase Detector Conditioning . . . . . . . . . . . . . . . . . . 93

4.10 .1 Preconditioning Rationale . . . . . . . . . . . . . 94

4.10.2 Implementing the Preconditioning Circuitry . . . . . . . . 96

4.11 Saving/Recalling closest digital state . . . . . . . . . . . . . . . 98 
4.12 Lock Position Initialization . . . . . . . . . . . . . . . . . . . . 99

4.13 Summary . . . . . . . . . . . . . . . . . . . . . . . . 99

5 PLL Example: Simulation and Measurement . . . . . . . . . . 101

5.1 Introduction . . . . . . . . . . . . . . . . . . . . 101

5.1.1 Debug, Test Structures and Other Circuitry . . . . . . . . 102

5.260 -Stage Cascaded-Pump x8/x32 PLL . . . . . . . . . . . . . 104

$5.2 .1 \quad$ PFD and Prefiltering . . . . . . . . . . . . 105

5.2 .2 Controlled Oscillator . . . . . . . . . . . . . . . 106

5.2.3 Top Level Specifications and Die-Photo . . . . . . . . . . . . 107

5.2 .4 Measured Transient Response . . . . . . . . . . . . . . 107

5.2.5 Jitter, Phase-Noise and Power Consumption . . . . . . . . . . 112

5.3 Conclusion . . . . . . . . . . . . . . . . . . . . . . . 114

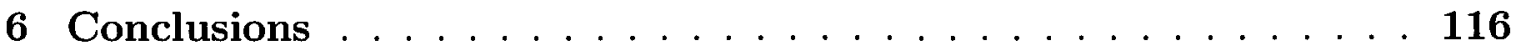

6.1 Summary . . . . . . . . . . . . . . . . 116

6.2 Contributions . . . . . . . . . . . . . . . . . . . . 119

6.2 .1 Associated research . . . . . . . . . . . . . . . 120

6.3 Publications . . . . . . . . . . . . . . . . . . . . 120

6.3 .1 Refereed . . . . . . . . . . . . . . . 120

6.3 .2 Patents/Patent Applications . . . . . . . . . . . . . . . . . 121

6.3 .3 Other . . . . . . . . . . . . . . . . . . 121

6.4 Future Work . . . . . . . . . . . . . . . . . . . . . . . . 122

Appendix A PLLs and DLLs in Clock Distribution . . . . . . . . . 124

A.1 Thesis Application: Digital Clocking _. . . . . . . . . . . . . 124

A.1.1 How Clock Delays lead to Circuit Failure . . . . . . . . . . . . 124

A.1.2 Conventional Clock Distribution . . . . . . . . . . . . . . . . . 124

A.1.3 Asynchronous Design . . . . . . . . . . . . . . . . . 128

A.1.4 Globally Asynchronous Locally Synchronous Systems . . . . . 128

A.1.5 Active Clock Synchronization with DLLs and PLLs . . . . . 129

Appendix B Further Simulation Results . . . . . . . . . . . . . 134

B.1 Overview . . . . . . . . . . . . . . . . . . . . . . 134 vii 
B.2 Charge Pump . . . . . . . . . . . . . . . . . . . . . . . . 134

B.2.1 Noise of the PFD, Prefilter and Charge-Pump . . . . . . . . 134

B.3 VCO Design, Range and Noise Characterization . . . . . . . . . 135

B.4 Filter Construction . . . . . . . . . . . . . . . . . . 136

Appendix C General PLL Design Procedure . . . . . . . . . . . 150

C.1 VCO Design . . . . . . . . . . . . . . . . . . . . . 150

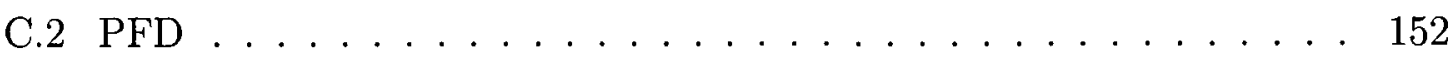

C.3 Charge-Pump . . . . . . . . . . . . . . . . . . . . 153

C.3.1 An Aside: UP/DN Mismatch and Compliance Range . . . . . 153

C.4 Charge Pump Current Sources . . . . . . . . . . . . . . . . 154

C.5 Charge Pump Switches . . . . . . . . . . . . . . . . . . 155

C.6 The Loop Filter . . . . . . . . . . . . . . . . . . . . 156

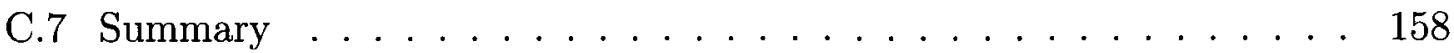

Appendix D Characterizing Jitter . . . . . . . . . . . . . 159

D.1 The Ambiguity of Jitter . . . . . . . . . . . . . . . . . . . 159

D.1.1 Period Jitter . . . . . . . . . . . . . . . . . . . . . . 160

D.1.2 Integrated Jitter . . . . . . . . . . . . . . . . 161

D.1.3 Linking Period Jitter and Phase Noise . . . . . . . . . 162

References ........................... 164 


\section{List of Figures}

2.1 PLL and DLL Models and Circuits . . . . . . . . . . . 8

2.2 DLL Edge combination Logic: An example . . . . . . . . . . . 9

2.3 Block diagram of general feedback system . . . . . . . . . . . 12

2.4 Open-Loop Bode plot analysis of PLL . . . . . . . . . . . . . 14

2.5 Describing Closed-Loop Transfer Function Graphically . . . . . . . . 18

2.6 Open/Closed loop transfer of VCO Referred noise . . . . . . . . . 20

2.7 Noise sources and transfer functions in a PLL . . . . . . . . . . . . . . . 21

2.8 Setting optimal loop bandwidth . . . . . . . . . . . . . . . . 22

2.9 PLL Alternative Control Structures . . . . . . . . . . . . . . . . 24

2.10 Example of an all-digital PLL (ADPLL) . . . . . . . . . . . . 28

2.11 Dual-Loop Architecture to reduce analog sensitivity . . . . . . . . 33

2.12 Digital Deskewing DLL as used in Intel Itanium, from Tam [1] . . . 36

2.13 Olsson's All-Digital PLL: Standard Implementation [2] . . . . . . . 37

2.14 Staszewski's All-Digital PLL: Very-low phase-noise, high complexity [3] 40

3.1 Cascaded Charge-Pump Architecture . . . . . . . . . . . . . . . 43

3.2 Cascaded Pump Formation . . . . . . . . . . . . . . . . . . . . . . . . . . . . . . 45

3.3 Cascaded Charge Pump . . . . . . . . . . . . . . . 46

3.4 Soft Control Handoff between control nodes . . . . . . . . . . . 48

3.5 Cascaded Charge-Pump DLL Example . . . . . . . . . . . . 49

3.6 Cascaded Charge-Pump in a PLL or DLL . . . . . . . . . . 51

3.7 System Level Testbed . . . . . . . . . . . . . . . . . . . . . 52

3.8 Equivalence of Low Gain Analog PLL and Cascaded Pump PLL . . . 53

3.9 Effect of Current Linearity on Acquisition . . . . . . . . . . . 54

3.10 VCO Gain and Noise . . . . . . . . . . . . . . . . . 55

3.11 System Simulator: Overview . . . . . . . . . . . . . . . . . . . . . 57

3.12 System Simulator: Parameters . . . . . . . . . . . . . . . . 59

3.13 System Simulator: Details . . . . . . . . . . . . . . . . . 60 
3.14 System Simulations: Main Result . . . . . . . . . . . . . . . 61

3.15 Simulation Speedup of System Level Simulator . . . . . . . . . . . 62

3.16 Simulation of Hi Gain VCO with Big Caps . . . . . . . . . . . . . 65

3.17 Noise Characterization of Lo Gain VCO . . . . . . . . . . . . . 66

3.18 Simulation of Hi Gain VCO with Big Caps . . . . . . . . . . . . 67

4.1 Tri-State buffer implementation of cascaded charge-pump . . . . . . . 69

4.2 Comparison of a regular and inverting thermometer code . . . . . . 70

4.3 DLL Configuration of an Inverting cascaded charge-pump . . . . . . 71

4.4 Removing redundant transistors in the cascaded charge-pump . . . . 73

4.5 Controlling VCOs and delay elements with a thermometer code . . . 74

4.6 Modeling Non-Ideal Charge-Pumps: $R_{C P}$ and Non-Linearity . . . . 75

4.7 Non-Ideal Charge-Pump Loop Effects . . . . . . . . . . . . . . 76

4.8 Cascaded Charge-Pump Transistor Arrangement . . . . . . . . . . . 78

4.9 Characterizing the Gain of the Cascaded Charge Pump . . . . . . . 79

4.10 Consistency of Cascaded Charge-Pump UP and DN Currents . . . . . 81

4.11 Loop Effects of partitioning the VCO control in Type II PLLs . . . . 85

4.12 Logic for filter rotation . . . . . . . . . . . . . . . . . 86

4.13 Digital Stabilization Logic . . . . . . . . . . . . . . . . 90

4.14 Cascaded charge-pump Leakage Suppression . . . . . . . . . . . . . 91

4.15 MOS Varactor Supply Sensitivity . . . . . . . . . . . . . . . . . 92

4.16 Conventional PFD . . . . . . . . . . . . . . . . . . . 94

4.17 Pulse Extension and Off-Level Rebiasing Circuits . . . . . . . . 96

4.18 Using pass-transistors to limit ON voltage levels . . . . . . . . . . . 97

4.19 Adjustable RC Prefiltering and Steering Logic . . . . . . . . . . . . 98

5.1 Die micro-graphs of $1^{\text {st }}$ and $2^{\text {nd }}$ prototypes. . . . . . . . . . . 101

5.2 Block Diagram of the 2nd Prototype . . . . . . . . . . . . . . 102

5.3 Software Control . . . . . . . . . . . . . . . . . . . . . . . . 103

5.4 Testbed Control . . . . . . . . . . . . . . . . . . . . . . . . . . . . . . 104

5.5 PLL Implementation . . . . . . . . . . . . . . . . . . . . 105

5.6 Simulated Charge-Pump Gain With/Without prefiltering . . . . . . . 106

5.7 Die Photo: Focus on region near PLL . . . . . . . . . . . . . . . . 108

5.8 Specifications: Simulated vs Measured Performance Summary . . . . 109 
5.9 Measured Transient Response of Shared Filter Sections . . . . . . . 110

5.10 Simulated Transient Response of Locking PLL . . . . . . . . . . . 111

5.11 Instability at low lock-voltages . . . . . . . . . . . . . . . . . 113

5.12 Measured Period Jitter and Wideband Phase-noise . . . . . . . . . 114

5.13 Phase-Noise Sim vs Measurement . . . . . . . . . . . . . . . . . . 115

A.1 Timing Violations due to Clock Skew . . . . . . . . . . . . . 125

A.2 H-Tree Distribution . . . . . . . . . . . . . . . . . . . . . . 126

A.3 A Globally Asynchronous Locally Synchronous Prototype . . . . . . . 130

A.4 Active DLL Clock Synchronization _. . . . . . . . . . . . . . 131

A.5 Using PLLs for Clock synchronization . . . . . . . . . . . . . . 132

B.1 Prefilter and Charge-Pump Response . . . . . . . . . . . . 137

B.2 Prefilter and Charge-Pump Noise Results . . . . . . . . . . . . . 138

B.3 Prefilter and Charge-Pump Noise Contributers . . . . . . . . . . . . 139

B.4 Prefilter and Charge-Pump Noise Contributers . . . . . . . . . . 140

B.5 VCO Construction and Range . . . . . . . . . . . . . . . . . . . 141

B.6 VCO Stage Details . . . . . . . . . . . . . . . . . . . . . . 142

B.7 VCO Power Consumption . . . . . . . . . . . . . . . . . . . 143

B.8 VCO Phase Noise . . . . . . . . . . . . . . . . . . . . . . . . 144

B.9 Filter's TX gate Resistance . . . . . . . . . . . . . . . . . . 145

B.10 Filter's resistance vs Switch resistance . . . . . . . . . . . . . 146

B.11 Filter Noise . . . . . . . . . . . . . . . . . . . . . 147

B.12 Filter Capacitance . . . . . . . . . . . . . . . . . . . . 147

B.13 Component Integration . . . . . . . . . . . . . . . . . . 148

B.14 Simulated locking over Process/Temperature . . . . . . . . . . . . 149

D.1 The inappropriate lexicon of Jitter $\ldots \ldots \ldots \ldots$

D.2 Period Jitter . . . . . . . . . . . . . . . . . . . . . . . . 160

D.3 Integrated Jitter . . . . . . . . . . . . . . . . . . . . . . . . . 161

D.4 Linking Period jitter to Phase Noise . . . . . . . . . . . . . . . 163 


\section{List of Tables}

2.1 Comparison of analog DLL/PLL implementations . . . . . . . . 35

2.2 Comparison of digital DLL/PLL implementations . . . . . . . . 38

2.3 Comparison of mixed-mode DLL/PLL implementations . . . . . . . 39

6.1 Comparison vs other low-complexity/power PLLs . . . . . . . . 118 


\section{Abbreviations, Definitions, Symbols and Acronyms}

ADC

ADPLL

ASIC

BW

BFOM

$C_{T}$

CAD

CCP

CP

CDR

$\mathrm{DAC}$

$\mathrm{dBc}$

$\mathrm{DCO}$

DL

DLL

DSP

ECC analog to digital converter

all-digital phase-locked loop

application specific integrated circuit

bandwidth, normally applies to $3 \mathrm{~dB}$, or half-power, frequency

Banerjee Figure of Merit: a common synthesizer figure of merit is the phase noise floor in-band, when referenced to a $1 \mathrm{~Hz}$ comparison frequency. To convert from a phase-noise (PN) figure in $\mathrm{dBc} / \mathrm{Hz}$ with $f_{\text {ref }}$ and $f_{v c o}, B F O M=P N_{f_{v c o}}-20 \log \left(f_{v c o} / f_{\text {ref }}\right)-10 \log \left(f_{\text {ref }}\right)$. The measured BFOM for this work is BFOM(10kHzoff set $)=-93-$ $20 \log (8)-10 \log (16 e 6)=-93-18-72=-183 d B c / H z$

total capacitance of the loop filter $\left(C_{1}+C_{2}+C_{3}+C_{4}\right)$

computer aided design

cascaded charge-pump - Refers to the integration circuit introduced in this thesis which generates a vector of thermometer-coded voltages rather than a single-voltage as in the conventional charge-pump

charge-pump

clock/data recovery

digital to analog converter

decibels relative to carrier

digitally controlled oscillator, equivalent to an NCO (A VCO with discrete digital settings)

delay-line

delay-locked loop

digital signal processing

error control coding 


\begin{tabular}{|c|c|}
\hline EDA & electronic design automation \\
\hline FIFO & first-in first-out \\
\hline FPGA & field-programmable gate-array \\
\hline FOM & $\begin{array}{l}\text { Figure of Merit: In this work it is normally the product of area }\left(m^{2}\right) \text {, } \\
\text { power }(m W) \text { and peak-to-peak Period Jitter }(p s) \text {. The FOM for this } \\
\text { work is } 0.07 \text {. }\end{array}$ \\
\hline G & forward loop gain \\
\hline GALS & $\begin{array}{l}\text { globally asynchronous, locally synchronous. A system integration } \\
\text { method where each subsystem is encapsulated in a wrapper that masks } \\
\text { the external asynchronous interface timing }\end{array}$ \\
\hline gate & $\begin{array}{l}\text { a logic-gate. Normally refers to the delay or area of a } 2 \text { input NAND } \\
\text { gate ( } 4 \text { transistors). It is useful to normalize delay/area across technol- } \\
\text { ogy nodes. In } 0.18 \mathrm{um} \text { TSMC CMOS, with the Virtual Silicon Tech- } \\
\text { nologies (VST) cell library, it consumes } 12.2 \mathrm{um}^{2} \text {. }\end{array}$ \\
\hline $\mathrm{H}$ & reverse loop gain \\
\hline HW & hardware \\
\hline jitter & $\begin{array}{l}\text { Time domain fluctuations of the clock's transition point away from its } \\
\text { ideal position. Jitter may be defined as either period jitter or integrated } \\
\text { jitter, and can be quoted as either an rms or peak number. Period jitter } \\
\text { looks only at the deviation of the clock edge relative to the preceding } \\
\text { cycle, and is important in digital clocking. Integrated jitter is the } \\
\text { deviation of the clock edge relative to an ideal signal of the same average } \\
\text { frequency beating in the background. Note that the Fourier transform } \\
\text { of the long-term jitter vs time is the phase noise spectrum. See also } \\
\text { Appendix } D \text {. }\end{array}$ \\
\hline$I_{C P}$ & charge-pump current \\
\hline $\mathrm{K}$ & gain (often applied with subscripts) \\
\hline$K_{C P}$ & Charge-pump gain $[\mathrm{Amps} / \mathrm{rad}]$ is proportional to charge-pump current \\
\hline$K_{V}$ & $\begin{array}{l}I_{C P} \\
\text { voltage-controlled oscillator/delay-line gain }([\mathrm{Hz} / \mathrm{V}] \text { for a } \mathrm{VCO},[\mathrm{sec} / \mathrm{V}] \\
\text { for a delay-line })\end{array}$ \\
\hline de & the end-point of a clock distribution tree - normally a flop-flop \\
\hline & loop filter \\
\hline
\end{tabular}


loop-BW Typically refers to the closed-loop bandwidth of a PLL/DLL (equivalent of $\left.\omega_{0 d B}\right)$.

M multiple of the reference clock in either a DLL or PLL. Is also the divisor in the feedback path of a PLL

MAP Maximum A-priori - refers to one of the algorithms used for errorcorrection in modern communication circuits

Marmoset nickname for the 1st prototype IC: a GALS DSP asic for software radio

MDLL Multiplying Delay-Locked Loop. A mix between a DLL and PLL where a ring-oscillator is occasionally re-seeded by a reference pulse

MiM Metal-Insulator-Metal: A special fabrication layer used to create lowleakage capacitances in analog and mixed-signal ICs.

$\mathrm{N}$

$\mathrm{NCO}$

$\mathrm{PD}$

PFD

PLL

PN number of stages in a cascaded charge-pump numerically controlled oscillator, equivalent to a DCO (A VCO with discrete digital settings)

phase detector

phase/frequency detector

phase locked loop

phase noise: normally quoted in $d B c / H z$ at a particular offset, or as an integrated number. Note that the integrated phase noise and rms integrated jitter are equivalent. For example, an RMS jitter of $2 \mathrm{ps}$ out of a 2 ns VCO period would result in an integrated phase noise of $20 \log (2 \pi * 2 p s / 2000 p s) \mathrm{dBc}$

PNoise Periodic Noise analysis - A simulation technique which simulates noise levels and transfer functions at various points in the cycle of a PSS solution (see below).

PVT process, voltage and temperature

PWM pulse-width modulated

PSS Periodic Steady State - An iterative transient simulation method which generates accurate voltage/current vs time results for large-signal periodic circuits.

$R_{C P} \quad$ the parallel output impedance of the current sources of the charge-pump (ideally $R_{C P}=\infty$ )

RMS root-mean-square of a sequence : $R M S=\sqrt{\text { average }\left(s(n)^{2}\right)}$ 
SERDES serial/deserialization

skew the difference in arrival time between related signals

slew The rise/fall time of a signal, normally measured between $10 \%$ and $90 \%$

SpectreRF Transistor-level circuit simulator developed/distributed by Cadence Design Systems

spurs Undesired signals which repeat in a deterministic fashion appear as distinct spikes in the frequency spectrum. This is in contrast to random noise (thermal, shot, flicker) which create a consistent noise floor. Common sources of spurs include reference feedthrough, and parasitic coupling through supplies, substrate and signal paths. The sources of these spurs in the frequency domain contribute (along with noise) to jitter in the time domain.

synthesizer industry jargon referring to a PLL/DLL system to generate signals of a certain frequency or phase. The term is often, but not universally, used to describe all of the PLL/DLL components with the exception of the VCO or delay-line.

Type-I PLL Phase locked loop with only a single pole at the origin (from the VCO)

Type-II PLL Phase locked loop with two poles at the origin (from the VCO, and CP integrator)

UI Unit-Interval: Used to normalize jitter results as a fraction of the symbol period. eg. For a 1000ps symbol period, 100ps of jitter is 0.1 UI.

$V_{c} \quad$ The effective control voltage on the tuning port of the VCO.

$V_{i} \quad$ A particular control voltage $i$, which is a component of $V_{c}$. Note that $\sum_{i=0}^{N-1} V_{i}=V_{c}$.

VCDL voltage controlled delay-line

VCO voltage controlled oscillator

Verilog an event-driven language suitable for digital designs and verification. Also known as Verilog-1995 or Vanilla verilog to differentiate it from Verilog-2001 and SystemVerilog which include more functionality.

Verilog-A an analog modeling language with syntactic similarity to Verilog-1995 (Vanilla verilog)

VLSI very large scale integration

$Z(s) \quad$ used to represent loop-filter impedance 


$\begin{array}{ll}\omega_{0 d B} & \text { unity-gain bandwidth is also the closed-loop bandwidth (or simply the } \\ & \text { loop-BW) of a PLL/DLL } \\ \omega_{n} & \text { undamped natural frequency of a second order system, is a measure of } \\ \text { bandwidth } & \text { used in this thesis to indicate the pole at } s=0 \text { inherent in the VCO } \\ \omega_{p 0} & \text { used in this thesis to indicate the pole near } s \approx 0 \text { due to the finite } \\ \omega_{p 1} & \text { impedance of the current sources of the charge-pump ( } \omega_{p 1}=1 /\left(R_{C P} *\right. \\ & \left.\left.C_{T}\right)\right) \\ & \text { used in this thesis to indicate the pole in the loop-filter caused by the } \\ & \text { stabilizing resistor }\left(R_{1}\right) \text { combined with the smoothing capacitor }\left(C_{2}\right) \\ \omega_{p 2} & \text { used in this thesis to indicate the stabilizing zero of the loop filter } \\ \omega_{z} & \left(\omega_{z}=1 /\left(R_{1} C_{T}\right)\right) \\ & \text { damping factor, a measure of stability in } 2 \text { nd order systems, should be } \\ \zeta & \approx 0.7 \text { for critical damping. }\end{array}$




\section{Chapter 1}

\section{Introduction}

Phase-locked loops (PLLs) and delay-locked loops (DLLs) are fundamental building blocks used in every area of electronics. They are used to synthesize clocks of various frequencies and/or phases. While RF communications is often the focus of research, several other applications also require clock generation and control circuitry, but have very different requirements. This thesis introduces a new synthesizer architecture focused on this secondary market, where the goals are very low area and power consumption.

\subsection{Applications of Phase and Delay Locked Loops}

\subsubsection{Synthesizers for wireless communications - Low Noise}

In RF communications, the purity of the synthesizer is defined in terms of phase-noise. The phase-noise can often dominate the various sources inside a radio, and therefore limit the achievable signal-to-noise ratio (SNR). In turn, the SNR determines the achievable modulation scheme and bit-rate. In the case of cellular communications, given the very low received signal strengths, the cost of radio spectrum and the need to support multiple simultaneous users with high data-rates, the RF synthesizer is typically designed to achieve very low phase-noise as a priority, at the cost of die-size, power consumption, and integration efficiency. Much of the research in phase-locked loop and delay-locked loops is aimed at these low-noise synthesizers. 


\subsubsection{Synthesizers for wired communications - High Density}

In other applications, such as wireline communications, the goals are quite different. Increasingly, vendors are relying on multi-channel high-speed serial links. For these, and similar applications, the purity of the synthesizer is often defined in terms of eyediagrams and jitter (rather than phase-noise) ${ }^{1}$. With larger signal strengths, more noise from the synthesizer can be tolerated. Also, unlike many RF radios, there may be multiple synthesizers or phase controllers inside an IC. Even then, they merely handle the IO, where the core function of the IC is something unrelated (eg. RAM, DSP, FPGA, etc.) The main goals of this type of synthesizer is to achieve very high density, consume little power, and require no external components - while maintaining an acceptable level of jitter (or phase-noise) for the application.

\section{Clock Distribution}

An extreme case of this second kind of synthesizer is in clock distribution. Ideally, the clock should arrive at all portions of an IC at the same time. Worsening process variations increase the error in clock arrival times, while higher clock speeds reduce the tolerance to this error. Phase-locked loops or delay-locked loops are ideally suited to remove this timing error by sensing the skew between clock arrival times and removing it.

Significant effort was spent investigating the issue of efficient clock distribution. This was intended as the primary application of this work and the reader is referred to Appendix A which describes the preliminary work in some detail.

\subsection{Goal: Small, Low Power Synthesizers}

The research started with an attempt to invent active clock alignment circuits only a few flip-flops big - making them effective for use in large scale clock-distribution systems. As the work developed, this ambitious goal was scaled back slightly (the PLL profiled in Chapter 5 is approximately 60 flip-flops in size, with DLL based deskewing elements about 20 flip-flops in size), but the application scope widened to

\footnotetext{
${ }^{1}$ Phase noise and jitter are essentially equivalent, but are specified in the frequency and timedomain respectively. See Appendix D for more information.
} 
include small and low-power synthesizers for use in clock-data recovery and similar applications.

\subsubsection{The Figure of Merit}

In keeping in line with the research intentions, it is useful to develop a quantitative measure for the success of the work. While there is a commonly used figure of merit (FOM) to measure the phase-noise performance of a synthesizer ${ }^{2}$, this does not take into account the efficiency of the design. For this purpose the author has introduced an alternate figure of merit, the areaøpower $\bullet i t t e r$ product $^{3}$. While area and power consumption are the focus of the work, gains in these areas should not come at an unacceptable cost in terms of jitter or phase-noise.

\subsection{Theme of Thesis: The Cascaded Charge-Pump (CCP)}

The new cascaded charge-pump (CCP) presented in the following chapters, replaces the charge-pump and filter structure in conventional DLLs and PLLs with a very compact multiple output charge-pump. As will be shown in Chapter 3 , it effectively reduces VCO gain $\left(K_{V}\right)$ without sacrificing range. The reduction in $K_{V}$ results in smaller, more practical filters, or it can be traded for increased charge-pump gain and better noise suppression ${ }^{4}$.

\subsubsection{Drastically Reduced Size}

DLLs and PLLs are normally too expensive to use extensively as one would a flip-flop or logic gate. For example, one of the most efficient DLL approaches targeting clock

\footnotetext{
${ }^{2}$ The Banerjee figure of merit (BFOM) [4] measures the phase-noise floor of the synthesizer (excluding the VCO), and normalizes it to a $1 \mathrm{~Hz}$ VCO and $1 \mathrm{~Hz}$ reference. See the glossary or references for more information.

${ }^{3}$ Peak-to-peak period jitter has been chosen for the figure of merit for two reasons. It is reported in the relevant literature more often than phase-noise or integrated long-term jitter, and it is arguably more relevent for SERDES and digital clocking applications. See Appendix D for more information regarding jitter variants.

${ }^{4}$ Improved noise suppression will also allow wider loop-BW and thus smaller filter size under most circumstances.
} 
distribution (depicted in Appendix A Figure A.4 from Kim [5]) consumes 6.4mW @ $2 \mathrm{Ghz}$, and 4600 'equivalent gates' of area for a single deskewing DLL, not including the capacitor of their loop-filter (which is typically dominant). It became the goal of this research, therefore, to architect a new type of deskewing DLL which was far more area and power efficient than the state-of-the art. With minor modifications, the invented structure was also found to be suitable for controlling PLL based synthesizers and alignment circuits.

As will be covered in Section 2.5, for a given loop bandwidth, the required capacitances in the loop-filter are proportional to the loop-gain, $K_{V} K_{C P}$ (VCO gain * charge-pump gain). As such, halving $K_{V} K_{C P}$ results in a halving of the capacitance requirements and thus filter size. It is not uncommon for the capacitor sizes to take over 10-20x the area of the PLL's active components (Maneatis [6] and Ahn [7] are examples). As always, in engineering it makes sense to tackle the greatest offender, and in this case it is the loop filter. By effectively reducing $K_{V}$, we reduce the circuit size.

\subsubsection{Improved Noise Suppression}

Normally, the dominant noise source inside the PLL loop bandwidth is contributed by the current sources in the charge-pump. If the charge-pump current $I_{C P}$ is increased, the noise contribution of the pump increases only by $\sqrt{I_{C P}}$. This results in a net improvement of signal-to-noise ratio, or in other terms, input referred noise, with an increase of charge-pump current and gain $K_{C P}$. If the noise from these current sources dominates, doubling $I_{C P}$ will reduce output noise by $3 \mathrm{~dB}$. Unfortunately, increases in $K_{C P}$ would require larger loop-filter components, which are to be avoided. By using the cascaded charge-pump, the gain reduction in $K_{V}$ can be traded for an increase in $K_{C P}$ without increasing the loop-filter size.

\subsubsection{Other improvements...}

In the conventional analog scenario, a single analog voltage controls the speed of the oscillator or delay-line. But, as is often cited [8] [9], lower supply voltages are reducing the available voltage swing of analog circuits. To maintain a suitable frequency range for the VCO or delay-line with a smaller control swing, its gain $K_{V}$ must be increased 
with the associated penalties. By implementing the control string with a vector of signals as is done in the cascaded charge-pump, $K_{V}$ can be chosen completely independently of the supply voltage, relieving designers and circuits of the burden of reduced supply swing.

It will be shown that the cascaded charge-pump shares many beneficial characteristics of all-digital PLLs (ADPLLs). Like ADPLLs, the CCP permits storage and recollection of the closest digital lock state, enabling quick reacquisition after idle periods or suspension of the input. Also, as technology scales, the CCP benefits from reduced transistor sizes nearly as well as fully digital versions. It can be implemented with either standard CMOS logic gates or custom transistor arrangements packaged as 'standard-cells' (both approaches have been used here), making it easy to integrate into digital VLSI circuits with automated implementation tools and no hand-layout (after construction of the initial standard-cell).

Unlike ADPLLs, however, the cascaded charge-pump is inherently an analog method and does not suffer from quantization induced jitter - caused when an oscillator or delay-line is forced to toggle between discrete settings above and below the ideal values. Furthermore, the CCP does not require time-to-digital converters, digital filters, explicit control storage, or decoding logic - making it significantly smaller and more power efficient than digital or dual-loop structures.

\subsection{Outline}

Chapter 2 provides background material regarding loop-theory and also contains a brief literature review - highlighting various analog, digital and mixed-signal DLL and PLL architectures. The targeted application is synchronization and high-speed serial communications within digital ICs. This necessitates very compact, low-power synchronizers and low integer- $\mathrm{N}$ frequency multipliers with moderate period jitter characteristics (eg. $<50$ ps peak-peak).

Chapter 3 discuses the cascaded charge-pump from a system-level perspective. Two system-level simulators have been written, and were used at various stages of the research to characterize aspects of the system. Though it has been intuitively discussed here, the simulation results of Chapter 3 will show the equivalence of an $\mathrm{N}$-stage cascaded charge-pump to a conventional single-stage analog loop with VCO 
gain $K_{V} / N$. It will then show via simulation how this facilitates a reduced filter size and/or better noise suppression via increased charge-pump gain.

Chapter 4 describes many of the circuit-level simplifications used to increase the efficiency of the architecture. Specifically, efforts have been made to reduce the area and power of the circuit, while improving flexibility. It goes on to discuss the effects of non-idealities on this architecture vs conventional single-voltage analog ones.

Chapter 5 presents measured results of the architecture used in a specific PLL circuit. It is compared to theory, measurements, and the state-of-the art.

Finally, Chapter 6 concludes with a brief summary, lessons learned, and a proposed list of future areas of exploration.

The reader is also encouraged to review the Appendices where there are two particular contributions of interest. Appendix $D$ has a unique treatment of jitter and its relationship to phase-noise, while Appendix C provides a step-by-step design method to produce efficient PLL circuits which meet a specified phase-noise mask. This set of guidelines can be used for both conventional analog loops, as well as with the cascaded charge-pump. 


\section{Chapter 2}

\section{Background}

\subsection{Overview}

This chapter introduces the PLL and DLL, highlighting their differences and the advantages and disadvantages of each in different applications. It provides a brief review of general loop-theory, and then more specifically applies the loop-theory to phaselocked loops. Unlike most mathematical treatments, there is a concerted attempt to apply a more intuitive and graphical explanation of the loop transfer functions. As in most analysis, the transfer function of the system with respect to the reference port and VCO output port are derived, and the implications of these transfer functions are explored with respect to chosing an optimal loop bandwidth. Ultimately, the loop bandwidth is normally chosen to optimize noise performance, and the size of conventional circuits is then dominated by the capacitance required to implement this bandwidth.

PLLs and DLLs are fundamentally mixed-signal in nature, but where the boundaries are may vary. A review of the three main architecture choices is presented along with a brief discussion of the implementation issues inherent in each type.

Finally, a literature survey tabluates a number of specific solutions, of each type, currently available in the literature.

\subsection{Basic PLL and DLL Operation}

In a PLL, Figures 2.1a and 2.1c, the negative feedback loop adjusts a voltagecontrolled oscillator (VCO) and forces the divided output phase $\left(\phi_{f d b k}\right)$ into alignment 


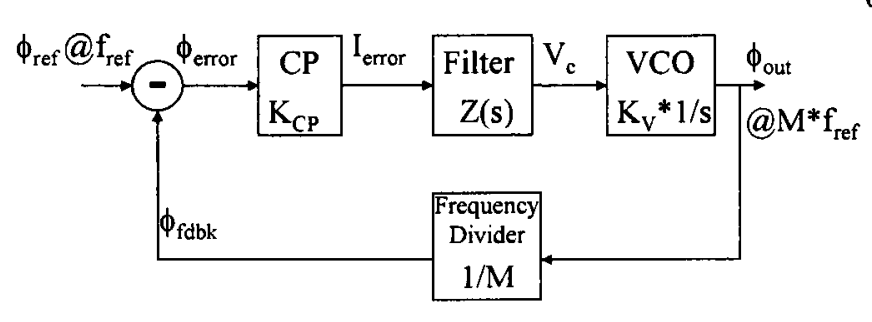

(a) PLL Model

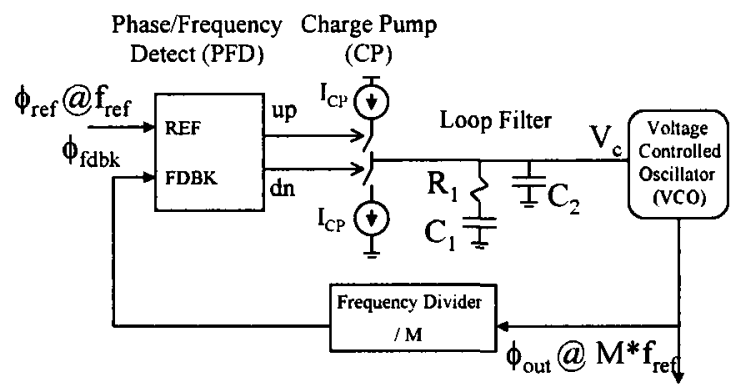

(c) A PLL Implementation

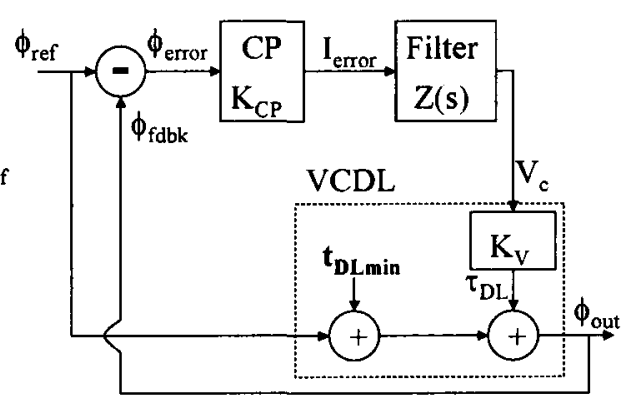

(b) DLL Model

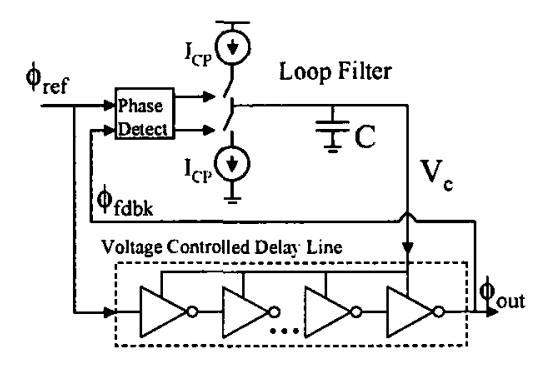

(d) A DLL Implementation

Figure 2.1: PLL and DLL Models and Circuits

with the phase of the reference signal $\left(\phi_{r e f}\right)$. If the phases are kept aligned, then the frequencies are identical, since even a slight frequency difference would immediately cause one signal to creep up on the other, disturbing the phase and forcing correction. Since the output of the frequency divider is at the same frequency as the reference, the input to the divider, which is also the output of the circuit, must be at a frequency $f_{\text {out }}=M \bullet f_{\text {ref }}$.

In a DLL, Figures $2.1 \mathrm{~b}$ and $2.1 \mathrm{~d}$, the negative feedback loop adjusts a voltage controlled delay-line (VCDL) to ensure that the phase of some output signal $\left(\phi_{f d b k}\right)$ is kept aligned with a reference $\left(\phi_{r e f}\right)$. Since the loop will adjust the phases to match regardless of extraneous conditions, the DLL can be very useful to synchronize clock trees without much regard to process, temperature, supply, and loading concerns. Often the reference signal itself is fed into the delay-line, as in the figure, and so the loop ensures a phase delay of $2 \pi$ through the circuit. ${ }^{1}$ Taking advantage of the

\footnotetext{
${ }^{1}$ Without special precautions a DLL will actually ensure an integer number of clock periods through the delay-line, for a phase delay of $k * 2 \pi$, where $k$ is any integer.
} 
controlled delay-line, phases of the clock signal can be tapped out of the line and used as a multi-phase clock source, or as shown in Figure 2.2, these phases can be combined to produce an output clock at some higher frequency.
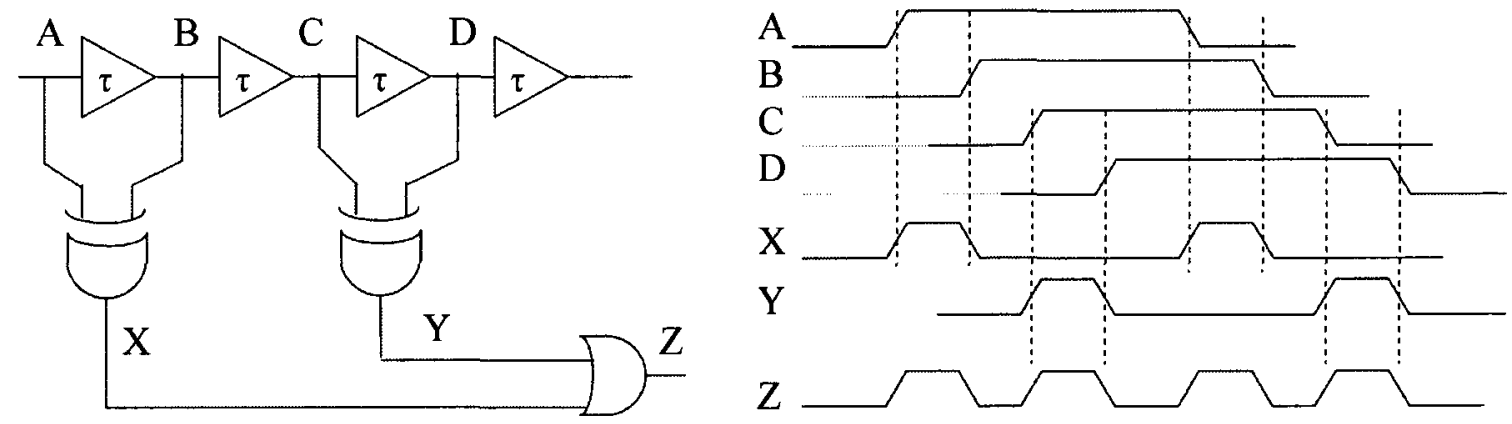

Figure 2.2: DLL Edge combination Logic: An example

\subsection{DLLs vs PLLs}

DLLs and PLLs have many things in common and can sometimes be used interchangeably. In almost all circumstances however, one is more suitable than the other. The fundamental difference is that a PLL contains an oscillator, whereas the DLL uses a controlled delay-line. The majority of this work focuses on PLLs due to their increased theoretical complexity, but various differences are highlighted here.

\subsubsection{Reference Noise}

In a DLL, the reference signal passes directly through the delay-line to the circuit output (Figure 2.1b), whereas in the PLL it is low-pass filtered and applied to a VCO, which isolates it from the output. In the DLL, all phase-noise on the reference passes through to the output, and further combines with any low-frequency contribution which, though phase shifted, makes it through the charge-pump/loop-filter. This means that a DLL has more phase-noise at the output port than at the input. This is in contrast to the PLL which can take in a noisy low-frequency reference, and because of the low-pass filtering, create a cleaner high-frequency output. In many cases where a DLL is used, the reference is considered to be relatively clean compared 
to other noise sources, and so this may not be an issue. In carefully designed clock distribution systems, the direct transfer of the reference noise through the DLL can be an advantage if the reference signal perturbations are kept synchronized across the system. That is, all clocks must arrive at the same time - even if they all happen to be a little late due to noise.

\subsubsection{Delay-Line Noise}

Noise sources and transfer functions will be further discussed in Section 2.6, but it will be shown that the feedback loop and filter work to suppress low-frequency thermal and flicker noise in either a VCO or delay-line. However, the noise in the delay-line tends to be lower than in a VCO, where the internal oscillator feedback accumulates noise each cycle. [10] It should also be noted here that the delay-line noise depends on its length. Noise in each stage accumulates to effect the final output phase. For uncorrelated noise sources, such as thermal and flicker, the addition of more stages has far less effect compared to correlated sources (such as supply noise). To reduce the effect of supply noise on DLLs, delay-lines should be kept as short, in terms of total delay, as possible. This means preference should be given to DLLs where high reference frequencies are available, such that $2 \pi$ of phase shift uses relatively few delay elements, or to 'deskewing' DLLs, where the delay-line does not need a full $2 \pi$ of phase-shift. ${ }^{2}$

\subsubsection{Clock Multiplication}

In a PLL, adjustment of the divisor can create any integer multiple of the reference frequency. For fractional multiples, it is possible to dither the divisor setting and let the loop-filter average the result. To create a higher frequency clock with a DLL, equally spaced phases of the reference must be created in the delay-line, and then these phases are logically combined to form higher multiples. If harmonic-free multiplication is required, or equivalently if the spacing between output clock pulses must be consistent, then the stages within the delay-line must be very carefully matched. It can quickly become area and power inefficient to implement DLL clock multipliers higher than $\mathrm{x} 3$ or $\mathrm{x} 4$.

\footnotetext{
${ }^{2}$ This is the approach used in Figure A.4b, as opposed to A.4a.
} 


\subsubsection{Clock Alignment}

Referring to Figure 2.1d, the loop forces the output phase of the DLL to match the reference. A clock distribution tree can be added to the output port, with the tree's output fed-back to the phase-detector instead, and the loop will work naturally to keep the tree end-point in phase with reference regardless of temperature, supply, and other fluctuations. This is the approach used in Figure A.4.

If, however, a DLL is used as a clock-multiplier, edge combination logic is necessary to manipulate the clock phases in the delay-line and produce the high frequency output. The output clock is thus offset from the reference by the delay of this logic (for example the delay of gates $\mathrm{X}, \mathrm{Y}$ and $\mathrm{Z}$ in Figure 2.2). Unfortunately, this delay is not controlled via feedback mechanisms and so the output clock phase is offset from the reference.

In the PLL of Figure 2.1c, the circuit output can be distributed via a clocktree, with an end-point of the tree feeding back and clocking the divider. The loop's feedback mechanism will ensure that the output of the divider is phase-matched to the reference. Fortunately, the divider delay can be well controlled (to match a standard flip-flop clk $\rightarrow Q$ delay) and can be compensated for to bring the divider's input $\approx$ in-phase with the reference port. This is in contrast to the edge-combination logic in a DLL where the delay is less predictable.

\subsubsection{Filter Stability}

Due to the VCO's $1 / s$ term in the Laplace model of the PLL (Figure 2.1a), there is a pole at $s=0$ in the open-loop transfer function, and an immediate phase shift of $-90^{\circ}$. This permits only $-90^{\circ}$ more phase shift in the system while the gain is above 1 , before the loop becomes unstable. ${ }^{3}$ This often requires special consideration in the design of the PLL loop filter whereas the DLL is stable with only a single-pole RC filter or integrator. There will be more discussion of stability in Section 2.4.1 when discussing loop-theory.

\footnotetext{
${ }^{3}$ This assumes that phase-margin guidelines are necessary and sufficient to ensure stability of the system, which is not always the case.
} 


\subsubsection{Comparison of Applications: DLL vs PLL}

At first glance, most of the DLL and PLL components appear identical. When considering the implementation details however, there are numerous differences. In DLLs there is a potential false lock problem where the delay-line might accidentally lock to a delay of $2 * T_{\text {ref }}$, or $3 * T_{\text {ref }}$, etc... rather than to $T_{r e f}$ as desired. Logic can be added to look for this condition and prevent it, but it adds to the gate-count and power consumption of the circuit. CMOS delay elements can experience wide delay variations across process and temperature conditions, and so, for clean wide range operation, delay-lines in DLLs must be made with great care and can consume significant resources. The high activity factor and loading through a DLL's delay-line contributes to relatively poor power efficiency compared to most PLL multipliers. To the DLL's benefit, because the filtering concerns are lower (and because the filter is often the dominant area burden in PLLs) the DLL can often be implemented in less area. If used in some deskewing circuits, such as Figure A.4b, a DLL's delay-line does not need wide range (or high gain), long depths, matched stages, or edge combination logic. Under these scenarios, the DLL can be made very efficiently in terms of both area and power consumption compared to a PLL.

\section{Summary}

DLLs are favored for deskewing applications, while PLLs are more suitable for high ratio (large M) clock multiplication.

\subsection{Loop Theory}

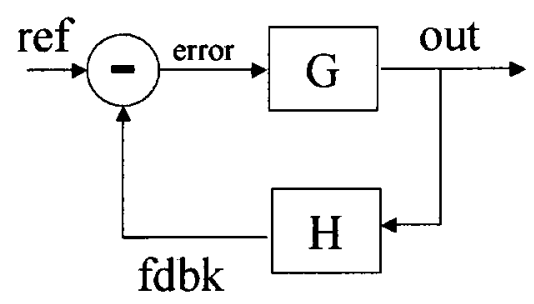

Figure 2.3: Block diagram of general feedback system 
Both phase and delay-locked loops are negative feedback systems that can be used for clock synthesis and alignment. To analyze these systems, a common approach is to break the loop into a forward path (designated $G$ ) and a reverse path (designated $H$ ). Where the loop is broken depends on the particular transfer function of interest. Given an open-loop transfer function $(G)$, and the feedback factor $(H)$, the closedloop transfer function of the system can be derived from the difference equation, and is:

$$
\frac{\text { out }}{\text { ref }}_{\text {closed-loop }}=\frac{G}{1+G H}
$$

In Equation 2.1, $\mathrm{G}$ and $\mathrm{H}$ can be complex or frequency dependent terms without loss of generality. This is the case in the typical PLL/DLL models of Figure 2.1 .

\subsubsection{PLL Open-loop Transfer Function}

In PLL design, arguably the frequency response of the system provides the best picture of overall operation. From the open-loop transfer function $\frac{\phi_{o u t}}{\phi_{r e f}}$, the unityfeedback bandwidth and stability of the PLL can be easily identified. Furthermore, an accurate representation of $\frac{\phi_{o u t}}{\phi_{r e f}}$ will show the higher order roll-off above the loop corner, providing some indication of the high-frequency noise suppression that can be expected. With the simplifying assumption that the divider $M=1$, an example Bode plot of an open loop $\frac{\phi_{o u t}}{\phi_{r e f}}$ characteristic is broken down in Figure 2.4. ${ }^{4}$

\section{Phase Frequency Detector and Charge-Pump}

A phase/frequency detector (PFD) measures the phase error (in radians) and a charge-pump (CP) converts the detected phase-error into a current, with gain $K_{C P}$

\footnotetext{
${ }^{4}$ In the Bode plots of Figure 2.4 and elsewhere, annotations will often show how the curves shift in proportion to $K$ or some other parameter. To be mathematically rigorous, because the curves are plotted in $\mathrm{dB}$, they should move in proportion to $20 \log (K)$. The $20 \log ()$ notation is dropped for simplicity and hopefully clarity. Also note that in these figures, and similar ones which follow in the thesis, the straight line approximations for both phase and frequency are strong simplifications intended for illustrative purposes. For example, in panel (b), the phase is shown to immediately flatten with a maximum of $-45^{\circ}$ between $\omega_{z}$ and $\omega_{p 2}$. In reality, since the slopes of the gain curves are not equal at $\omega_{z}$, a more accurate phase analysis would continue to show the phase approach a peak of $-20^{\circ}$ before retreating. For the sake of this thesis, however, these refinements are unimportant.
} 


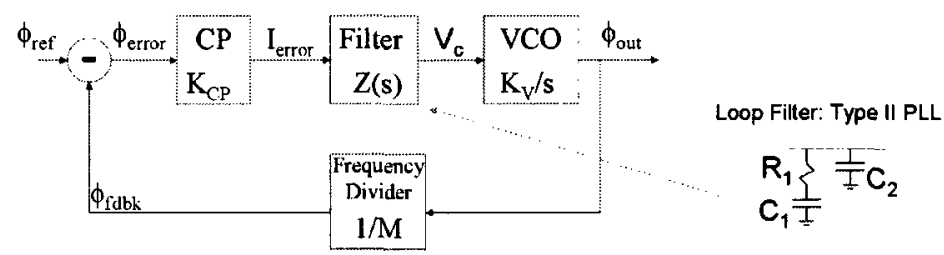

(a)

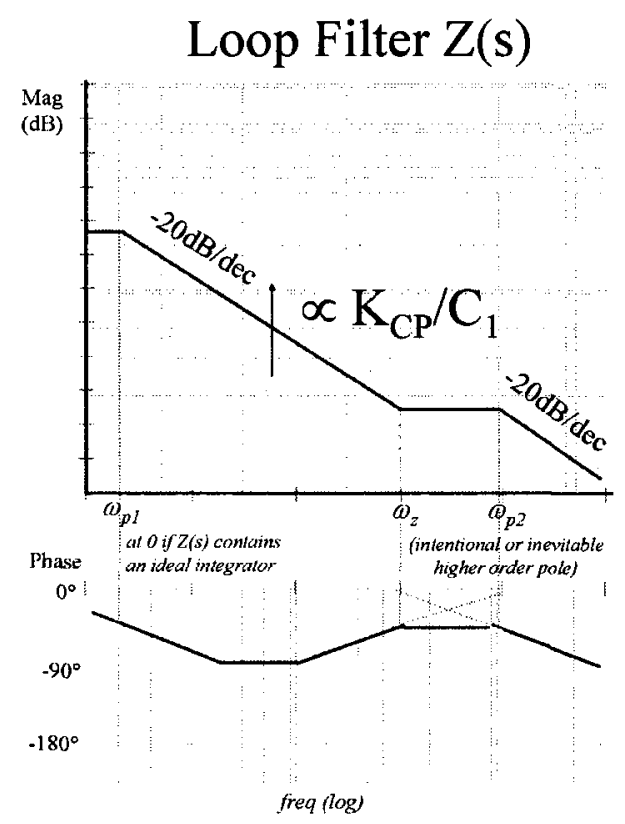

(b)

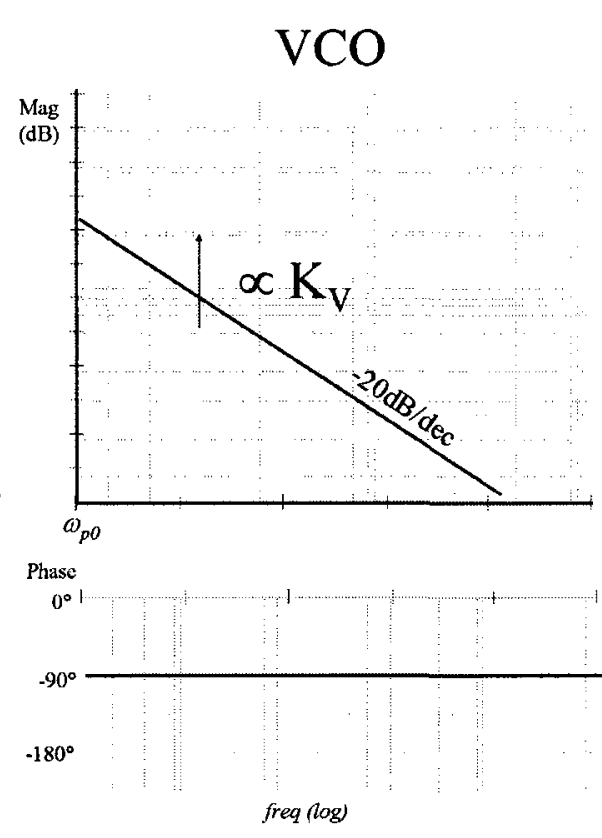

(c)

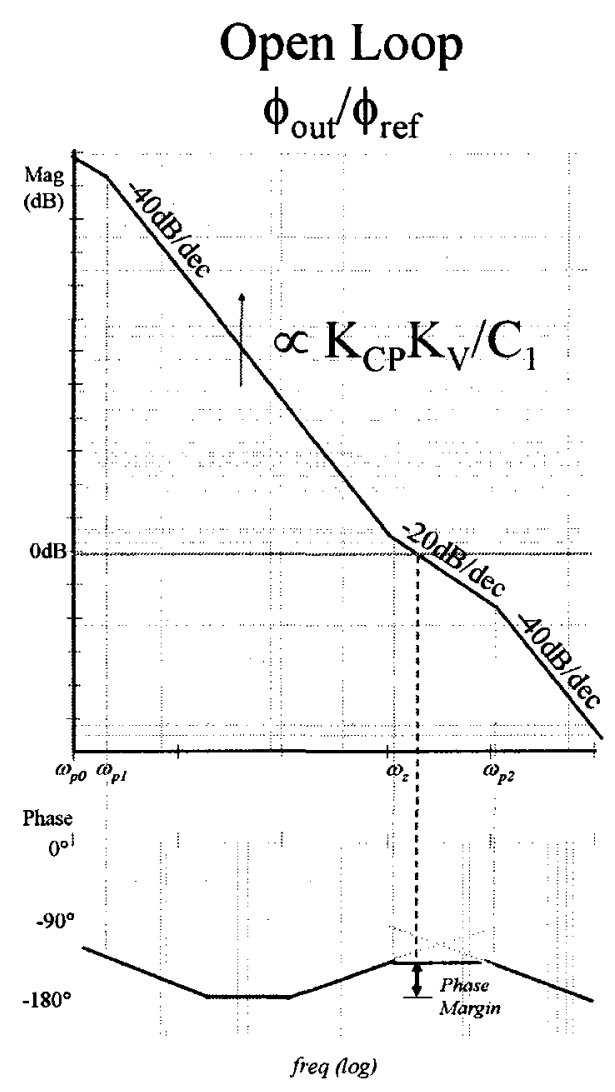

(d)

Figure 2.4: Open Loop Analysis of PLL using bode plots a) The PLL model b) The typical charge-pump and loop-filter combination have a pole at $\omega_{p 1}=1 /\left(R_{C P} C_{T}\right) \approx 0$, where $C_{T}=C_{1}+C_{2}$, a zero at $\omega_{z}=1 /\left(R_{1} C_{1}\right)$ and another pole at $\omega_{p 2}=1 /\left(R_{1} C_{T}\right)$. The absolute level of the curve scales with the ratio of $K_{C P} / C_{T}\left(\approx K_{C P} / C_{1}\right.$ since $C_{1}>>C_{2}$ ). c) The VCO has a pole at $\omega_{p 0}=0$ due to the conversion of frequency to phase. Its level scales with $K_{V}$. d) The combination of the CP, Loop-filter and VCO produce the open loop characteristic shown in $\mathrm{d}$. When the magnitude of the curve crosses 1 , or $0 \mathrm{~dB}$, the phase must be less than -180 degrees to ensure stability.

[A/rad]. The charge-pump is often modeled as two ideal current sources and two switches, as shown in Figure 2.1c. 


\section{$\mathrm{VCO}$}

The loop-filter integrates the charge-pump current and creates a voltage $\left(V_{c}\right)$ to control the VCO. The VCO has a gain of $K_{V}[\mathrm{MHz} / \mathrm{V}]$. Since $V_{c}$ adjusts frequency, but the loop works on phase information, $V_{c}$ must be integrated to convert to phase. The integration is modeled by a $1 / s$ term in the Laplace domain. In practice, this integration provides an additional low-pass filtering effect, along with an associated phase shift of $-90^{\circ}$ (Figure 2.4c).

\section{Loop Filter}

The loop-filter, Z(s), converts the charge-pump current to a voltage for the VCO. Typically, a filter such as that in Figure 2.1c is used, which consists of an integrator with a pole near the origin $\left(\omega_{p 1} \approx 0\right)$, a stabilizing zero at $\omega_{z} \approx 1 / R_{1} C_{1}$ and a higher order pole at $\omega_{p 2} \approx 1 / R_{1} C_{2}$. The loop-filter is driven by a current source, which has an ideal output impedance of $R_{C P}=\infty$. For practical sources, the finite output impedance of the charge-pump will combine with the capacitance of the loop-filter and move the pole $\omega_{p 1}$ from 0 to $1 /\left(R_{C P} * C_{T}\right) \approx 0$ as shown in Figure 2.4b [10]. ${ }^{5}$

\section{Open Loop Transfer Function}

Taken together, the open loop transfer function is pictured in Figure 2.4d and given in Equation 2.2.

$$
G={\frac{\phi_{\text {out }}}{\phi_{\text {ref } O L}}}=K_{C P} K_{V} Z(s) / s
$$

If using the typical loop-filter of Figure 2.4a:

$$
\begin{aligned}
\frac{\phi_{\text {out }}}{\phi_{\text {ref } O L}} & =\frac{K_{C P} K_{V}}{C_{T}} \frac{\left(1+s / \omega_{z}\right)}{\omega_{p 0} \omega_{p 1}\left(1+s / \omega_{p 2}\right)} \\
& \approx \frac{K_{C P} K_{V}}{C_{T}} \frac{\left(1+s R_{1} C_{1}\right)}{s^{2}\left(1+s R_{1} C_{2}\right)}
\end{aligned}
$$

\footnotetext{
${ }^{5}$ PLLs with a loop-filter pole at $\omega \approx 0$ are sometimes referred to as Type II, since they have 2 integrators - one in the loop filter and one in the VCO.
} 
A summary of the poles and zeros is as follows:

$$
\begin{aligned}
C_{T} & =C_{1}+C_{2} \\
\omega_{p 0} & =0,1 / s \text { from } \mathrm{VCO} \\
\omega_{p 1} & \approx 0,1 / R_{C P} C_{T} \text { from charge-pump } \\
\omega_{z} & \approx 1 / R_{1} C_{T} \approx 1 / R_{1} C_{1} \\
\omega_{p 2} & \approx 1 / R_{1} C_{2}
\end{aligned}
$$

An important point to remember from Equation 2.3 is that with this filter, the open-loop transfer function moves up and down with the ratio of gain to filter capacitance $\frac{K_{C P} K_{V}}{C_{T}}$. (See Figure 2.4d.)

\section{Stability}

In most feedback situations, when there is unity gain around the loop it is critical that the feedback signal is subtracted from the input to maintain 'negative feedback' and prevent instability. If $M=1$ (no frequency divisor), the $0 \mathrm{~dB}$ line of $\frac{\phi_{o u t}}{\phi_{r e f}}$ in Figure $2.4 \mathrm{~d}$ also corresponds to the unity gain point around the loop. The distance between $-180^{\circ}$, where the sign of the feedback signal changes, and the phase when the magnitude crosses the $0 \mathrm{~dB}$ line $\left(\omega_{0 d B}\right)$ is called phase margin and provides an indication of how stable the system is.

It is important to note that if the stabilizing zero at $\omega_{z}$ were not there, the phase would inevitably be at or below $-180^{\circ}$ at the unity gain frequency and the system would be unstable. $\omega_{z}$ 's purpose is to prevent this. For the most stable operation, either $\omega_{p 1}>\omega_{0 d B}$ (which will be shown to increase VCO noise contributions) or, more conventionally, $\omega_{z}<<\omega_{0 d B}$ and $\omega_{p 2}>>\omega_{0 d B}$. That is, the zero and higher-order pole should form a window around the $0 \mathrm{~dB}$ frequency. Spreading the window out provides a wider frequency range where the phase margin is close to $90^{\circ}$. In further sections it will be shown that opening this window is a trade-off - reducing the roll-off of VCO noise (if $\omega_{z}$ is too low) or reference noise and spurs (if $\omega_{p 2}$ is too high). It should also be mentioned that the gain $K_{C P} K_{V}$ has an effect on stability because its adjustment shifts the $\left|\frac{\phi_{o u t}}{\phi_{\text {ref }}}\right|$ curve up/down and changes the location of the $0 \mathrm{~dB}$ 
frequency. Normally $K_{V}$ is fixed by the application, and so a combination of $K_{C P}$ and $\mathrm{Z}(\mathrm{s})$ manipulation are used to shift $\omega_{0 d B}$ toward some optimal point.

\subsubsection{Closing the Loop}

Given the feedback Equation 2.1, repeated in Figure 2.5a for convenience, the loop can be broken into a forward path $(G)$ and reverse path $(H)$ as identified by the dashed lines. The immediate transfer function of interest is the closed-loop response of the output vs input, or $\frac{\phi_{o u t}}{\phi_{\text {ref }} \text { closed-loop }}$. For this transfer function, the forward path $G$ is chosen to correspond to the open-loop characteristic $\frac{\phi_{o u t}}{\phi_{r e f}}$, derived in Figure 2.4d, and the reverse path $H$ is chosen as the path through the divider $1 / M$.

Though the open-loop equations for $G$ and $H$ can be substituted into Equation 2.1 to provide a mathematical description of the closed-loop transfer function, such a function does not provide a very intuitive vision of the characteristic.

By examining the limiting cases of Equation 2.1, a natural picture of the closedloop characteristic emerges and is illustrated in Figures 2.5b for the unity feedback case $(H=1)$, and $2.5 \mathrm{c}$ where some divisor is used. First, if $G H>>1$, which is true at low-frequencies, then $\frac{\phi_{\text {out }}}{\phi_{\text {ref }} \text { closed-loop }}$ simplifies to the constant $1 / H$ which is the divider setting. For $G H<<1$ (at higher frequencies), then $\frac{\phi_{\text {out }}}{\phi_{\text {ref }} \text { closed-loop }}=G$, and the closed-loop characteristic follows the open-loop one. The frequency at which $|G H|=1$ is the unity loop-gain frequency $\left(\omega_{0 d B}\right)$ and is the point where the closedloop characteristic is crossing over from curve $1 / H$ to $G$. This point also corresponds to the closed-loop bandwidth of the PLL ( $\left.\omega_{\text {closed-loop }}\right)$.

The unity loop-gain frequency $\left(\omega_{0 d B}\right)$ is also critically important from a stability perspective. If phase shift around the loop has caused a sign change on $G H$ when $|G H|=1$, then the denominator of Equation 2.1 goes to 0 and the system becomes unstable. This is the intuitive justification for the use of phase-margin which measures how close the system gets to this limit. As evident in Figure 2.5c, increasing the divisor pulls $\omega_{0 d B}$ lower when compared to $2.5 \mathrm{~b}$ and will effect phase-margin - either increasing it or decreasing it depending on its position between $\omega_{z}$ and any higher order poles. 


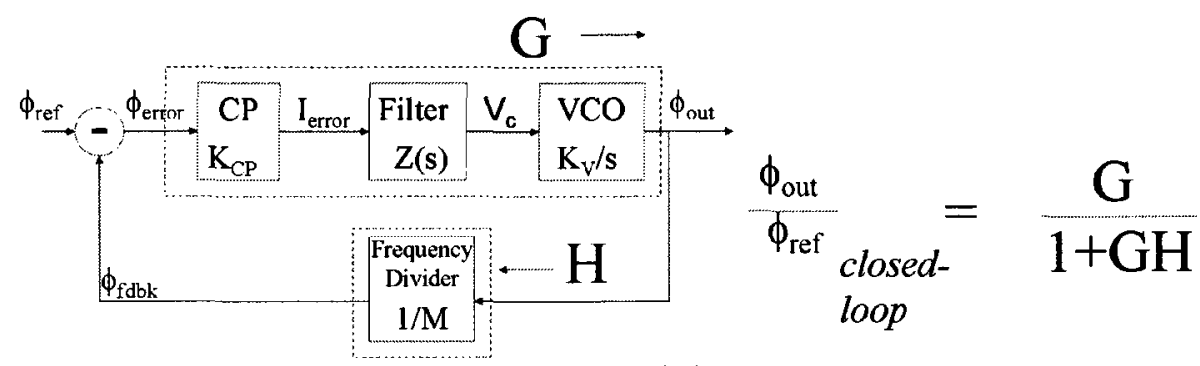

(a)

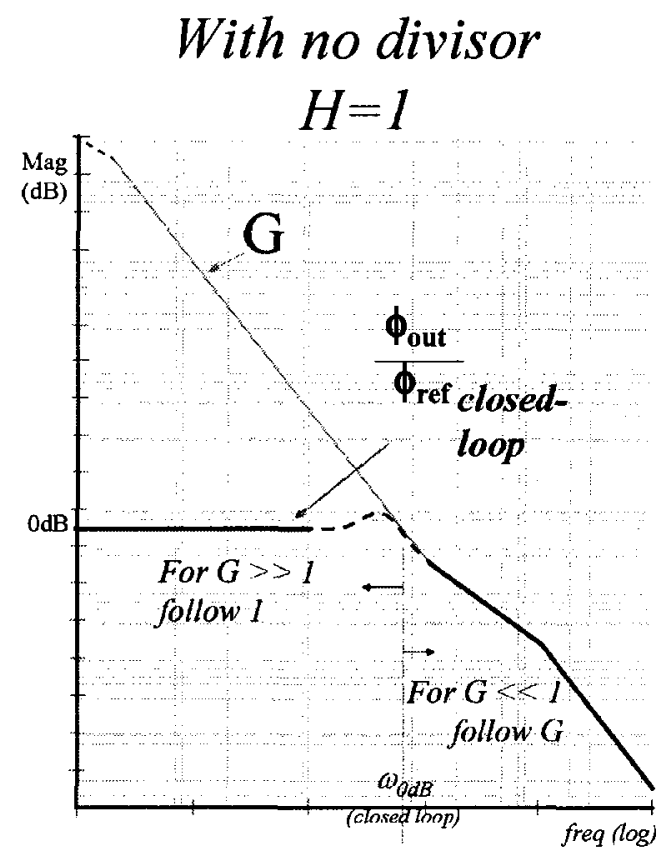

(b)

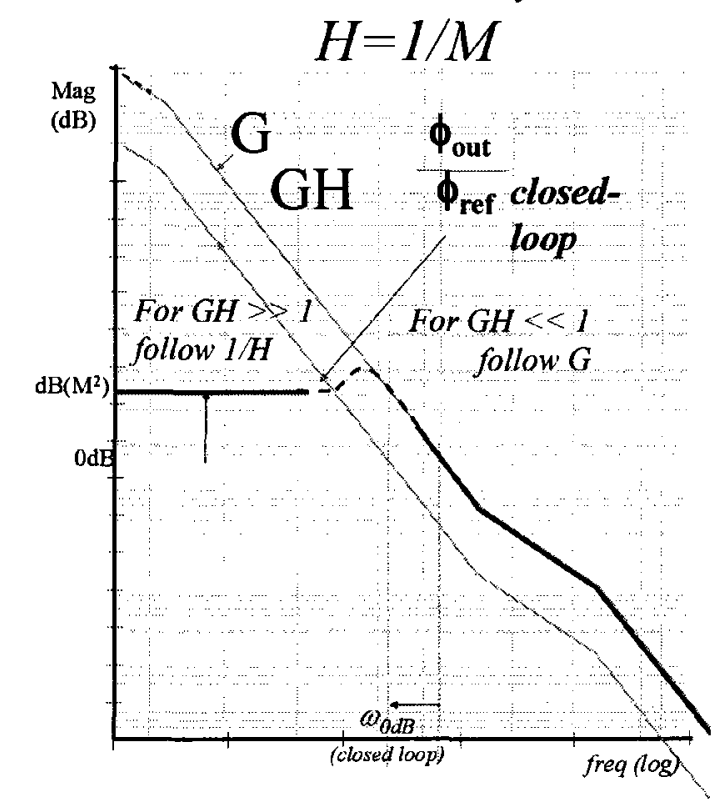

(c)

Figure 2.5: Open-Loop to closed-loop transfer function $-\phi_{\text {out }} / \phi_{\text {ref }}$ : Given that the closed-loop transfer function is $C L=G /(1+G H)$, For $G H>>1$ which is true for low frequencies, $C L=G / G H=1 / H=M$ and the input phase-noise transfers to the output, scaled by the divide ratio. For $G H<<1$, which occurs at high frequencies, $C L=G$, and the closed loop response follows the open loop response. The transition between the two asymptotes depends somewhat on the stability of the solution, with an example shown as a dashed line. A more mathematical, rather than figurative plot, is given in Chapter 3, Figure 3.10. 


\subsection{Effect of Loop gain on Filter size}

Referring to Figure 2.5b, the closed loop bandwidth of the PLL occurs when $G H=$ 1. Assume for simplicity that $M=1$, then the closed-loop bandwidth is simply determined when Equation $2.3=1$. Note the constant $K_{V} K_{C P} / C_{T}$. To keep the loop bandwidth constant, decreasing the VCO gain should be followed with an equivalent decrease in capacitance. This is the primary advantage of the cascaded charge-pump structure. Since it effectively reduces $K_{V}$ by $\mathrm{Nx}$, where $\mathrm{N}$ is the number of stages in the cascade, the capacitance requirements would also be, ideally, reduced by $\mathrm{Nx}$ for a substantial area savings.

\subsection{Noise Sources and Transfer Characteristics}

Noise can and will corrupt signals throughout the PLL. Transfer functions can be developed from each node to the output, but this is burdensome and, in a linear system, is unnecessary. Instead, noise sources at any point in the loop can be theoretically shifted around the loop (with the appropriate mathematical scaling) and treated as though the disturbance was caused on some other node. Commonly, the VCO noise is referred to the output port (at $n_{V C O}$ in Figure 2.7), and the other noise sources are scaled appropriately and referenced to the PLL input port (at $n_{r e f}$ ). The transfer function to reference referred noise at $n_{r e f}$ follows a low-pass characteristic, and was derived in the previous section (Figure 2.5). The VCO referred noise derivation is shown in Figure 2.6.

Figure 2.7, shows a summary of many of the different noise power-spectral densities (PSDs) in the loop, and how they are referred.

Equations 2.10 and 2.11 detail the reference and VCO noise transfer functions mathematically, and can be compared with their graphical representations. The conclusion is that low-frequency VCO noise is rejected by the loop, whereas high-frequency reference noise/information is rejected. The cutoff of these two filters is identical, and so there is a trade-off between suppressing VCO noise compared to most other noise sources in the system. 

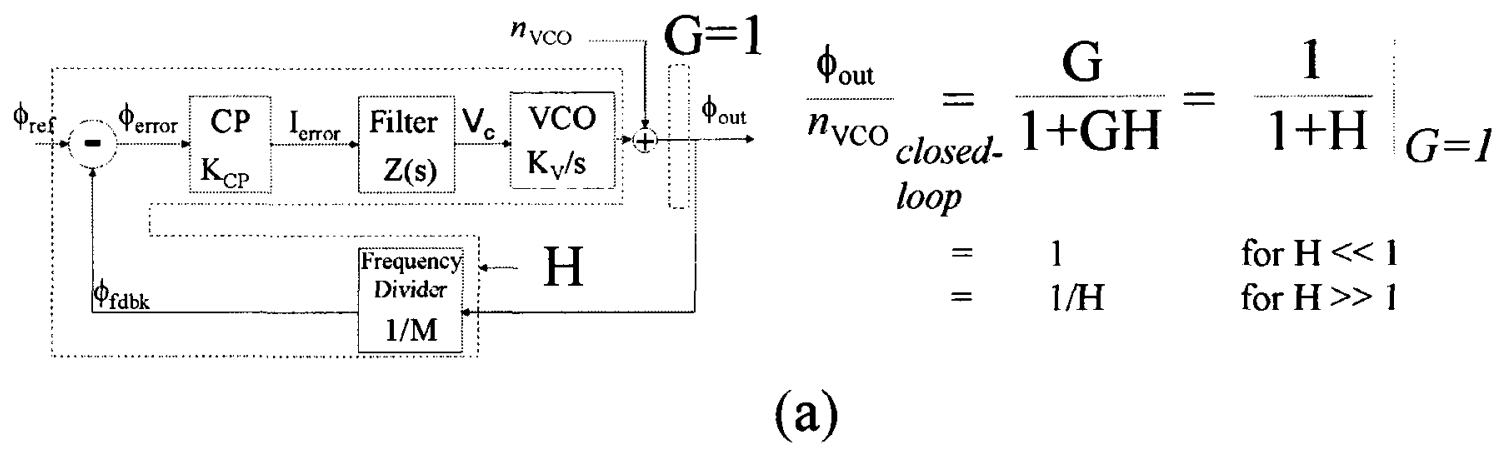

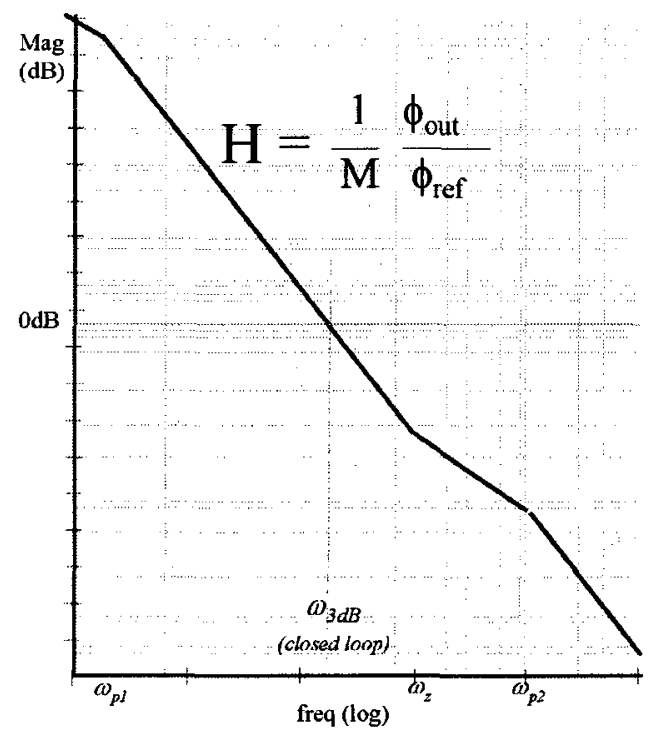

(b)

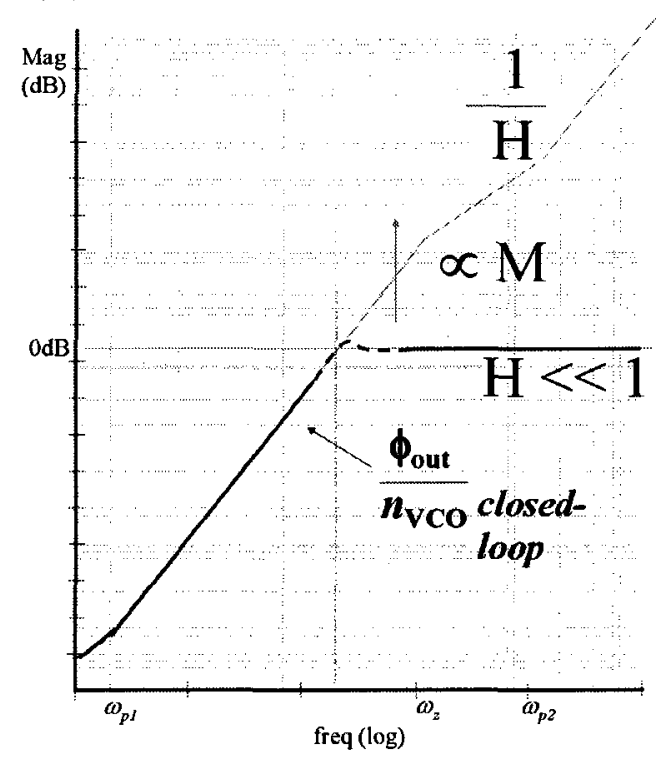

(c)

Figure 2.6: Open/Closed loop transfer of VCO Referred noise: Since the output port is directly connected to the $\mathrm{VCO}$, the forward gain $G=1$. The reverse path remains $H=\frac{1}{M} \frac{\phi_{\text {out }}}{\phi_{\text {in }} \text { open-loop }}$, regardless of where we analyze the loop. For $|G H|>>1$, which applies for low frequencies within the loop BW, $\frac{\phi_{\text {out }}}{n_{V C O} \text { closed-loop }}=1 / H$ and the VCO noise is suppressed. At higher frequencies, such that $|G H|<<1$, the transfer function is unity and VCO noise (or VCO referred noise) passes directly to the output.

$$
\begin{aligned}
& \phi_{\text {out }} / n_{\text {ref }}=20 \log 10\left(\frac{K_{C P} K_{V C O} * Z(s) / s}{1+K_{C P} K_{V} * Z(s) / M}\right) d B \\
& \phi_{\text {out }} / n_{\text {vco }}=20 \log 10\left(\frac{1}{1+K_{C P} K_{V} * F(s) / M}\right) d B
\end{aligned}
$$




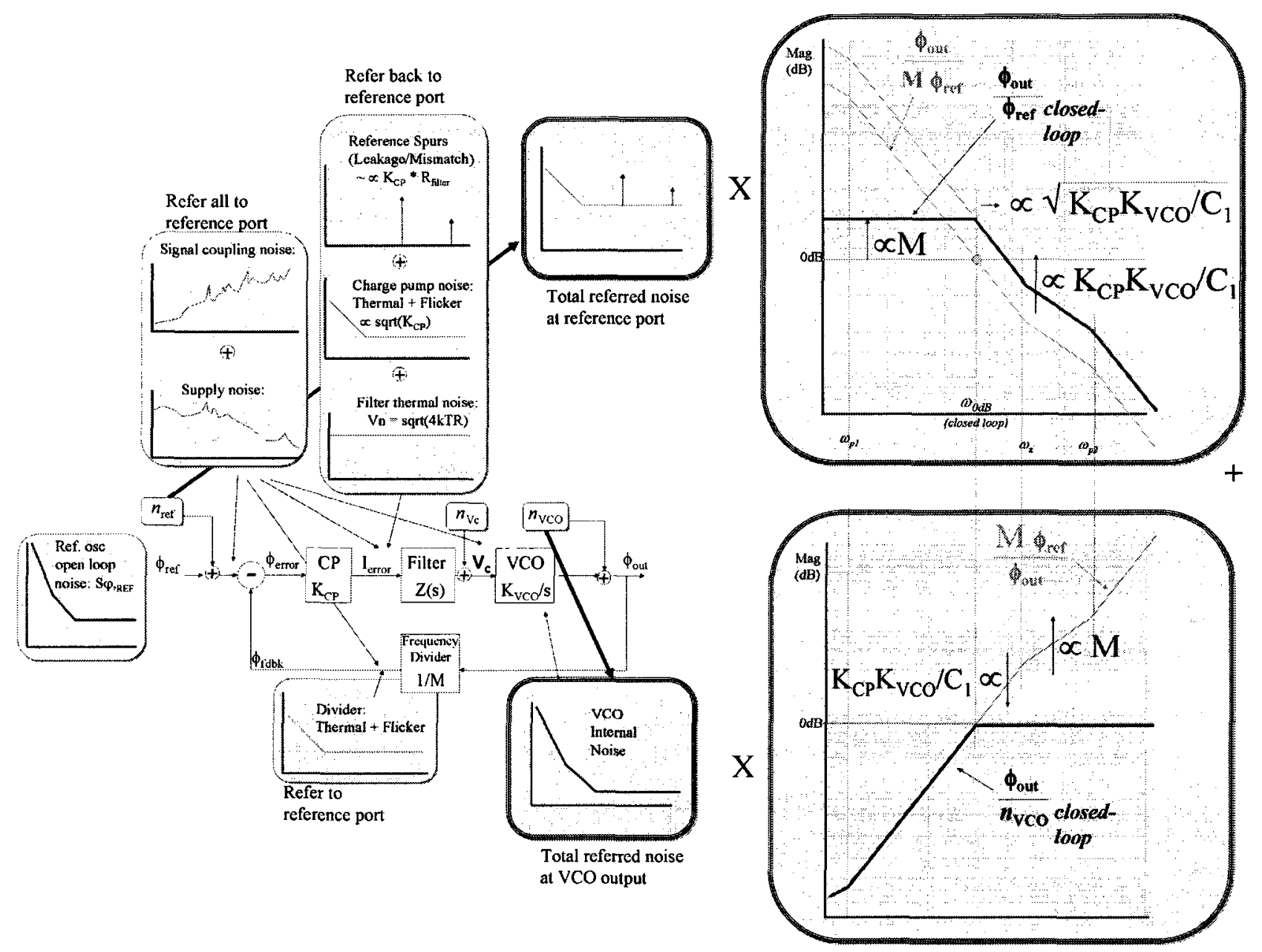

Figure 2.7: Noise occurring at various nodes in the PLL is typically input or output referred, allowing the designer to apply either the low-pass reference, or high-pass VCO noise transfer function.

\subsubsection{Optimal Loop Bandwidth}

Given the low frequency VCO noise rejection, and the high frequency reference path noise rejection, a few important observations can be made. At frequencies above the loop bandwidth, the VCO should dominate the phase-noise performance, and for frequencies below the loop bandwidth, the synthesizer ${ }^{6}$ should dominate.

\footnotetext{
${ }^{6}$ In a slight misnomer, but in keeping with industry nomenclature, the Synthesizer is a common term for all the components of a PLL other than the VCO.
} 
Figure $2.8^{7}$ shows the simulated phase-noise contributions of the charge-pump, loop-filter and VCO of the design detailed in the appendix. The optimal setting for the loop bandwidth is where the synthesizer noise (where the CP typically dominates) matches the VCO noise, as shown in $2.8 \mathrm{~b}$. If the bandwidth is set too low, as in $2.8 \mathrm{a}$, the VCO noise dominates the performance in-band, and characteristic 'bunny ears' appear. This is an indication of a noisy VCO, and that the loop bandwidth should be extended to suppress it. If the loop bandwidth is set too wide, as in Figure 2.8c, then the PLL suffers the synthesizer noise out to a wider bandwidth than is necessary.

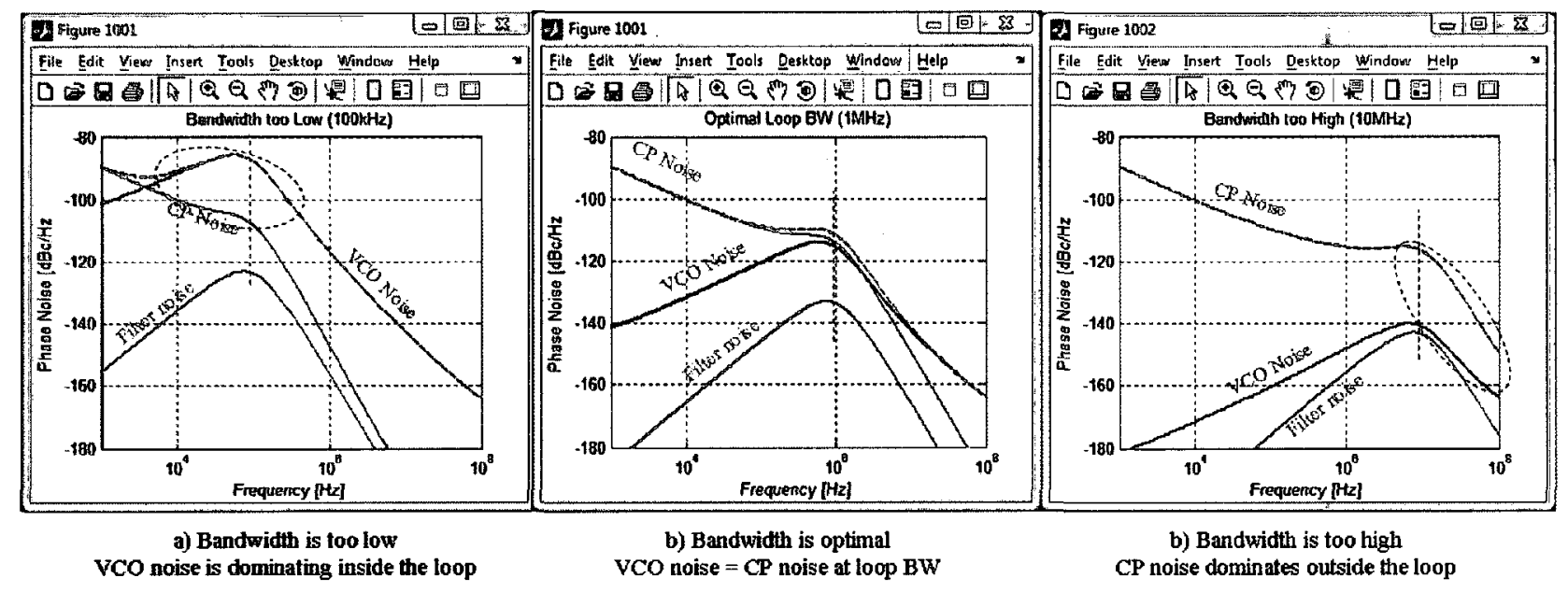

Figure 2.8: Setting the optimal loop bandwidth: The loop bandwidth should be set at the point where the open-loop charge-pump noise matches the open-loop VCO noise, as in (b). Too low and the VCO dominates in band, too high and the loop suffers the charge-pump noise out to a wider band-width than necessary to suppress the VCO.

\subsubsection{Increasing $K_{C P}$ for better noise performance}

Looking at Figure 2.8b, below the loop bandwidth, the dominant noise source is the charge-pump current sources. This is typical of PLLs. For every doubling of chargepump gain, however, the phase-noise contribution of these sources go down by $\approx 3 \mathrm{~dB}$. Unfortunately, all things being equal, this would also require an increase in the size of the filter capacitances to maintain the same loop-bandwidth. If the gain of the $\mathrm{VCO}$

\footnotetext{
${ }^{7}$ Credit goes to Hittite Microwave and Kashif Sheikh for the software used here to superimpose various open-loop noise transfer functions and optimize the closed-loop bandwidth.
} 
is scaled down, however, the charge-pump gain can be scaled up by an equivalent amount, and the filter does not need to change.

\section{Two-for-one: Better phase-noise and smaller component sizes}

A very interesting thing happens if we now re-consider the optimal loop-bandwidth. With $K_{V}$ scaled down by $10 \mathrm{x}$ (for example), $K_{C P}$ can scale up by $10 \mathrm{x}$, and there will be a $10 \mathrm{~dB}$ improvement in the in-band performance. ${ }^{8}$ Since the synthesizer is now a better performer relative to the $\mathrm{VCO}$, the loop-BW should be extended for the optimal phase-noise solution. With a $-20 \mathrm{~dB} / \mathrm{dec}$ slope on the $\mathrm{VCO}$, and a $10 \mathrm{~dB}$ improvement in the charge-pump noise, this translates to a $3.3 \mathrm{x}$ increase in the new optimal bandwidth. Quite fortunately, the capacitance sizes in the loop filter scale proportionally to $1 / B W^{2}$, and so opening up the loop by $3.3 x$ reduces the capacitance requirements by $10 \mathrm{x}$. Not only has the PLL become a better noise performer, but the passive requirements have been lowered by virtue of opening up the loop BW.

\subsection{Architectural Overview}

\subsubsection{Analog, Digital or Mixed-Signal}

A PLL or DLL are almost always mixed-signal in nature, but where the analog/digital boundaries are can vary depending on the architecture. One way to classify them is based on how the oscillator or delay-elements are controlled. Three options are shown in Figure 2.9, where the oscillator of a PLL can be controlled by an analog voltage, a digital string of bits, or by some combination of the two. Regardless of the approach, the dominant area cost, for integrated solutions, is in the filtering structure which takes input from the PFD and delivers the control to the oscillator.

While most of the discussion will focus on PLLs/DLLs of the analog variety, digital and mixed-signal structures are also gaining popularity. As will be discussed in the following sections, analog solutions suffer mainly from noise, repeatability and integration problems, whereas digital solutions suffer from quantization effects. In either case, the circuits tend to be quite large and inefficient from an area perspective.

\footnotetext{
${ }^{8}$ Assuming noise is dominated by the current sources of the charge-pump, as is typical.
} 

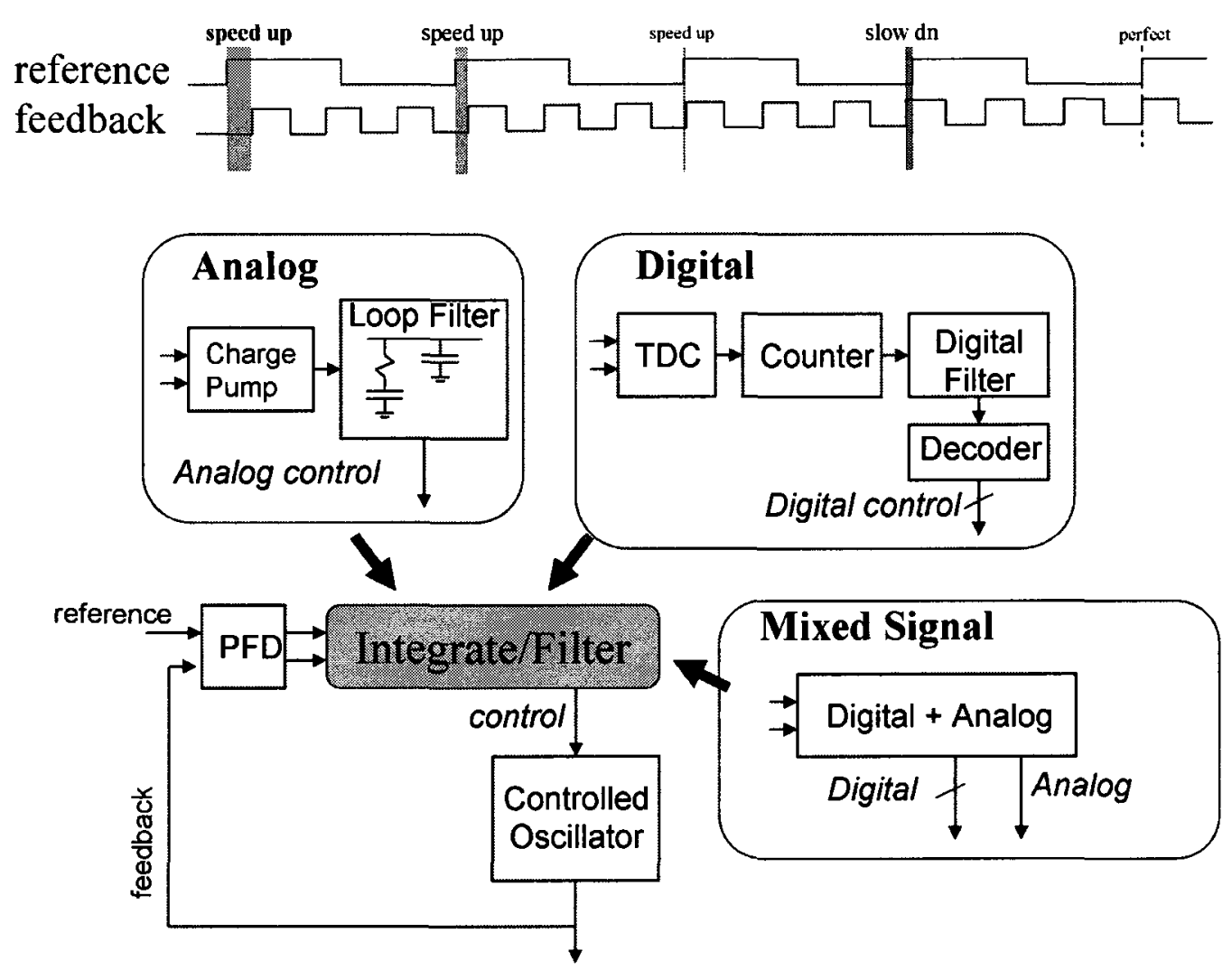

Figure 2.9: In the PLL, a phase-frequency detector (PFD) senses any phase offset between a reference signal and the divided output of an oscillator. It issues corrections into the loop and adjusts the speed of the oscillator until the PFD inputs are aligned in phase and frequency. The oscillator can be controlled by either an analog voltage (a voltage-controlled oscillator or $\mathrm{VCO}$ ), a digital string of bits (a numerically controlled oscillator or $\mathrm{NCO}$ ), or by some combination of the two (also typically called a VCO). In either case, the circuit size is typically dominated by the control structure which takes input from the PFD, filters it, and applies a control voltage to the VCO.

\subsubsection{Analog Implementation Challenges}

There are a number of issues which make analog implementations challenging. The cascaded charge-pump (CCP), to be covered in further chapters, intends to address a number of these issues: 


\section{Challenges addressed by the CCP in this thesis}

- Filter Size: Referring back to Figure 2.5, the loop BW is approximately set when $K_{C P} * K_{V} * Z(s) /(M * s)=1$. For a typical loop filter configuration, the natural frequency can be estimated as in Rogers, Plett and Dai [11], as $\omega_{n}=\sqrt{\frac{I_{C P} K_{V}}{C_{1} M}}$. Also from [11], with near critical damping and neglecting the higher order pole, the loop-bandwidth is then $B W[H z] \approx 2.4 \omega_{n} / 2 \pi$. Solving for the size of the main integration capacitor, and often then for the size of the design, $C_{1}=\frac{5.8 * K_{V} I_{C P}}{N *(2 \pi B W)^{2}}$. To achieve low loop bandwidths with large $K_{C P}$ (for low noise) and large $K_{V}$ (to satisfy range requirements), also requires very large capacitances. For example, to achieve a loop BW of $100 \mathrm{kHz}$, with $K_{V}=$ $100 \mathrm{MHz} / \mathrm{V}, K_{C P}=1 \mathrm{~mA}, M=8$, this estimate would require $C_{1} \approx 182 \mathrm{nF}$, which is unachievable for an integrated solution. The main feature here, is that the required capacitance is proportional to loop-gain, and inversely proportional to the square of the loop-BW. Doubling the loop-BW makes the filter $4 \mathrm{x}$ smaller, while halving the loop-gain halves the filter size.

- Pump Noise: In-band, the flicker noise of the charge-pump tends to dominate the overall PLL performance. To reduce the effect of pump noise, the transistors can be made larger and the pump current, $I_{C P}$, can be increased. Although the flicker and shot noise power of the pump increase with $10 * \log \left(I_{C P}\right)$, the signal power increases by $20 * \log \left(I_{C P}\right)$ and so a net gain in SNR can be achieved with more current. The cascaded pump structure will effectively lower $K_{V}$ and increase charge-storage capacity without a significant area overhead, thus permitting larger pump currents before loop-BW limitations and component area restrictions become prohibitive.

- VCO Range: As available supply voltages are reduced, the sensitivity of the VCO $\left(K_{V}\right)$ must be increased to maintain a certain output frequency range. This typically increases the noise generated by the oscillator, and also makes the entire loop more sensitive to 'mid-stream' noise (CP and filter noise), which is scaled by the VCO gain before reaching the output. The cascaded pump will be shown to remove control-swing limitations by extending the VCO control 'horizontally' to multiple nodes, as is done for digital control, rather than 'vertically' into the supply limit. 
- State Recollection: Though not as large a problem as the aforementioned issues, digital implementations have the advantage that they can store the control setting for the VCO. This permits seeding the control line for faster acquisition, and faster relock after idle periods. With analog implementations, ADCs and DACs are necessary to support this feature. The presented structure will be shown to allow partial state storage and recollection.

- Integration/Layout Constraints: In addition to the size of the filter, the analog components in a charge-pump/filter are typically quite large to achieve suitable matching and noise performance. As mentioned, often an off-chip filter is also necessary for tight loop bandwidths. In contrast to digital PLLs, which are tolerant to transients and coupling, analog layouts require significant isolation. The cascaded charge-pump in this thesis is designed for automated placement and routing with digital standard-cells, simplifying integration.

\section{Challenges not addressed by the CCP in this thesis}

- Dead-Zone: Due to finite turn on/off times of the current sources in the pump, it can not naturally respond to very small phase errors. To compensate, both the UP and DN current sources in the pump turn on for at least a fixed amount of time, and the difference between the charge is what is integrated into the loop. During these 'dead-zone avoidance pulses', since the current sources must always be on for some minimum amount of time, one gets increased pump noise at the output during lock.

- Static Mismatch: During the dead-zone avoidance pulses, any mismatch in the current sources creates a net charge accumulation or void on the VCO control port. The loop compensates by forcing a static phase offset that is large enough to offset the error. This static phase offset, followed by an effective current leak (due to mismatch while on), creates very short duration sawtooth pulses every reference cycle, which manifest as reference spurs (and their multiples) at the output.

- Dynamic Mismatch: While CP designers often verify the static matching of the UP and DN current sources to within 1\% error (even accounting for process 
mismatch), dynamic effects such as charge feedthrough on differently sized gates will tend to dominate the effective charge-mismatch, and therefore the static phase error and reference spurs.

- Charge-Pump Sampling Effects: The PFD and CP produce quick pulses of current, with a width proportional to the sampled phase-error. This is inconsistent with the otherwise continuous system. Though it can be modeled with z-transforms, as has been done in Gardner [12] and elsewhere, more often the phase-detector/charge-pump combination is modeled using the Continuous Time Approximation [12] [4] [13], which assumes that, as long as the bandwidth of the system is much smaller than the reference frequency (normally $<1 / 10^{t h}$ ), the discrete current pulses can instead be modeled as a continuous current which is proportional to the phase error at all times. This constraint however, forces a limit on the maximum loop-bandwidth for a given reference frequency. If the system remains linear, then the sampling does not create problems, however, it should be noted that by forcing a large amount of peak current for a short duration, stresses the linearity of the circuity (pump and VCO) more-so than a moderate application of current in a continuous fashion.

- Leakage: Charge leakage from the VCO tuning port, board dielectric, chargepump switches or elsewhere creates a drop in voltage which must be replaced by the loop for steady state operation. Leakage on the tune line generates a sawtooth waveform with a duty cycle extending the entire reference period, unlike with mismatch related issues which have far shorter duty cycles.

\subsubsection{Digital Implementation Overview}

In the analog DLLs/PLLs considered thus far, the oscillator or delay elements are ultimately controlled by a voltage stored on a large capacitance. This analog voltage is susceptible to leakage and to a host of noise sources (thermal, flicker, substrate and coupling) which degrade the quality of the output signal. As supply voltages are reduced, this noise becomes a more significant fraction of the overall control voltage and the output worsens. In digital PLLs/DLLs, instead of an analog voltage, a digital vector of bits controls the oscillator or delay-line. An example of an all-digital PLL (ADPLL) is shown in Figure 2.10. 


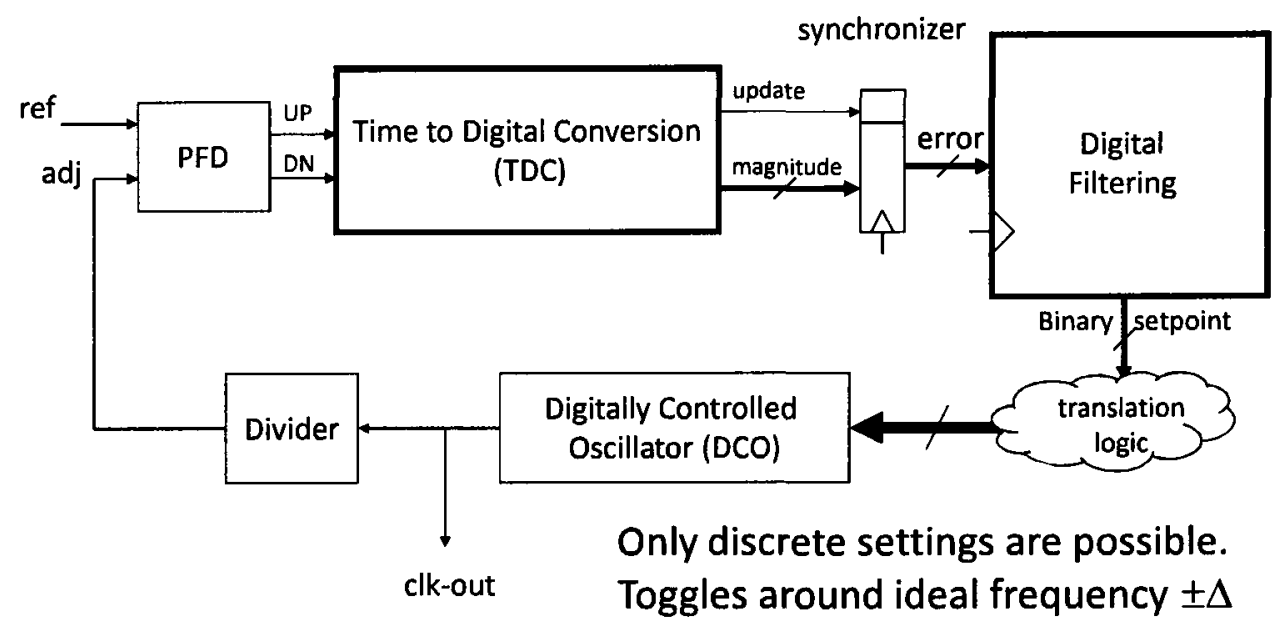

Figure 2.10: Example of an all-digital PLL (ADPLL)

These digital DLLs/PLLs mirror the construction of their analog counterparts. The digital loops can use a conventional PFD, but the UP/DN signals are fed into a digital circuit where their occurrences may be averaged over time (and the magnitude of the phase error is discarded) [14] [1], super-sampled by a high speed clock [15], or processed with a time-to-digital converter $(\mathrm{TDC})^{9}[2]$ [3]. These three approaches are similar, but offer various levels of accuracy in quantizing the phase error.

With any of these methods, the resultant phase error is then a digital signal, and is processed by digital FIR or IIR filters to perform the averaging. Since it is difficult to accurately implement delay elements with binary weighting, the output from the filter is often decoded into a form suitable for direct application to the delay elements (eg. a thermometer code), or potentially sent through a DAC for analog application to the oscillator or delay-line. ${ }^{10}$ In the following sections, the properties of 'all-digital' PLLs are explained in slightly more detail.

\footnotetext{
${ }^{9}$ Olsson [2] uses the abbreviation T2 $d$.

${ }^{10}$ If the output of the DAC is a voltage, this last approach is counter productive since a primary motivation for using the digital approach is to remove the limitations on control voltage swing.
} 


\subsubsection{Digital Implementation Challenges}

\section{Quantization Jitter}

Since the control of the oscillator or delay-line has discrete settings, it is unlikely to exactly match the desired output frequency/phase. The control word will toggle between values $\pm \Delta$ around the lock point, where $\Delta$ is the minimum delay step. This leads to quantization induced jitter, which degrades the quality of the output signal. This is the main problem with digital loops, but it can be mitigated by making the step-size very small, and/or dithering the effect to high frequency (where it is suppressed somewhat by the $1 / \mathrm{s}$ of the VCO) at the cost of added circuit complexity.

\section{Non-Monotonic Jitter or Instability}

The toggling nature of the control word also highlights another potential problem. If the delay of the oscillator/delay-line were not monotonic with the control signal, severe jitter may result. If a binary weighted delay element is implemented poorly, two adjacent control words (eg. $0111_{b i n}=7_{d e c}, 1000_{b i n}=8_{d e c}$ ) may vary in the opposite direction than is expected. The feedback of the loop will compensate somewhat for non-linear behaviour of the control string [2], but non-monotonic behaviour or severe non-linearity will likely result in instability. This is one of the reasons that controlled delay elements are typically implemented with thermometer coding [1] as opposed to binary weighting.

\section{Time-to-Digital Converter Resolution}

During lock, the up/down correction pulses from the phase/frequency detector would ideally be only a few ps wide. The time-to-digital converter is responsible for measuring this pulse width and providing the information to the downstream digital filters.

Inaccuracy in measuring the phase-error can treated with standard quantization theory [16] where, if the samples are uncorrelated from each other, the quantization noise can be modeled as having a flat power-spectral density. The level of this quantization noise is inversely proportional to the number of quantization levels. From the discussion of input referred noise in Section 2.6, the quantization noise will be scaled by the $\frac{\phi_{o u t}}{\phi_{\text {ref }} \text { closed-loop }}$ characteristic and appear at the output. Ultimately, 
provided a stable lock can still be achieved, the phase-error quantization noise causes poor phase-noise and jitter performance. [3]

The simplest time-to-digital converter is a 'bang-bang' phase-detector[17]. These are essentially binary time-to-digital converters, where they merely sense which direction to correct and feed this information into the loop.

The assumption that the quantization noise has a flat power-spectral-density is not necessarily valid for slowly changing signals, since there is correlation between the errors from sample-to-sample [16]. Since phase-error should change very slowly, some architectures take advantage of this and use sub-sampling - only updating the loop after a number of reference periods. This is done in the example of the Intel Itanium in Figure 2.12. For increased accuracy, a similar approach averages a number of PFD outputs before applying the result to the main loop-filter every few reference cycles. The disadvantage of this approach however, is that it "introduces a large loop delay which degrades DPLL [digital PLL] stability and severely limits the achievable closed loop bandwidth." [15].

\section{Dead-Zone}

A problem related to the time-to-digital converter is an increased dead-zone. The resolution of non-binary time-to-digital converters is typically ${ }^{11}$ limited by the delay of an inverter. In $0.18 \mathrm{um} \mathrm{CMOS}$, this is $\approx 50-60 \mathrm{ps}$. The result, is that for phase errors below this, the loop will not respond. In PLLs, since oscillator fluctuations within this dead-zone cannot be compensated by the loop, it results in higher phasenoise and increased jitter. In DLLs, such a large dead-zone may disqualify these circuits since phase alignment in the range of a few ps is often required.

\section{State Memory}

A disadvantage of analog implementations, is that if the DLL or PLL is powered down or the input signals are suspended, the control voltage will discharge and the frequency is lost, making reacquisition time consuming. This makes analog implementations relatively ineffective in digital clock multipliers and deskew elements where

\footnotetext{
${ }^{11}$ This resolution can be increased by using TDCs where a difference is taken between a pair of slightly mismatched delay-lines. This is sometimes referred to as a Vernier delay-line, and it comes at a significant cost in complexity.
} 
clock-gating may interrupt the reference signal for extended periods, and yet quick reacquisition time is also a priority.

For VLSI clocking purposes where clock gating may interrupt the input signal, a significant advantage of digital architectures is that the delay of the circuit is uniquely controlled by a digital control string stored in a set of registers. Since the lock-state of the circuit is in memory, the inputs can be suspended and frequency lock can be quickly recovered. Unfortunately, while the frequency control word is unique and can be restored quickly, the PLL must still regain phase-lock, which will be governed by the loop dynamics and typically proceeds no faster than an initial phase-lock. Whether phase lock is required, and the tolerances on frequency and/or phase accuracy to be considered locked vary widely and are governed by the application where the PLL is used.

\section{Noise Susceptibility}

Aside from VCO noise, which also exists in digital PLLs, the oscillator control voltage $V_{c}$ is of particular importance. In digital implementations there is a vector of control voltages, but each is held at binary 1 or 0 . Since no values are in an analog range, they are less susceptible to leakage and device noise (since $I_{D}=0$ ). Though digital outputs are sensitive to noise on the supply rails, the oscillator or delay-line can be designed with low sensitivity to these fluctuations. Unfortunately, as mentioned before, since the oscillator or delay-line can only be set to discrete values, it is prone to toggle between settings which are too-high and too-low of the ideal setting, introducing quantization induced jitter and creating an output of far lower quality than well designed analog implementations.

\section{Implementation Efficiency}

It is important to recognize that even in supposed 'all-digital' PLLs and DLLs, the VCO or delay-line and time-to-digital converter are still inherently analog components which will suffer from all sorts of noise (supply, coupling, thermal, flicker). Nevertheless, they can often be created with logic gates found in any digital standard-cell library.[2] These standard-cell digitally-controlled oscillators (DCOs), in combination with regular CMOS control logic, are portable and their area and power scale well 
across technologies. Their standard-cell design also allows circuit construction using digital design flows, where CAD tools automatically perform the majority of layout and routing tasks in the final construction of an IC. The 'standard-cell' compatibility of these implementations is a great advantage in reducing design and implementation time.

Unfortunately, from an area and power perspective, digital implementations often consume more resources than their analog counterparts. This is due to the relatively large complexity of the filters, decoders, and storage registers needed to control the loop. But, as technology scales, the digital implementations' efficiency improves more than the analog ones. A summary of various implementations found in the literature will be presented in Section 2.8.

\subsubsection{Mixed-Signal PLLs/DLLs}

In mixed-signal DLLs/PLLs, a combination of analog and digital approaches is used. A coarse digital word may be used to select a range of operation, and then fine analog control is used to narrow in on the particular lock point. An example of such a system is shown in Figure 2.11. In this manner, there is much more flexibility to reduce the analog VCO or delay-line gain $\left(K_{V}\right)$, and thus reduce the filter size and potentially the charge-pump noise contributions. In the conventional approach to this architecture, both a digital and analog control loop are necessary, and so it is sometimes referred to as a dual-loop architecture.

Unfortunately, there are limits to the $K_{V}$ reductions which are possible with this approach. In most applications, it is expected that a loop should be able to lock at one temperature extreme, and to maintain lock as the temperature fluctuates to the opposite extreme. The analog range in a dual-loop approach must be large enough to satisfy this. In addition to the temperature coverage problem, the disadvantage of the dual-loop architectures are the added power, area, and design complexity of the two-pronged attack. 


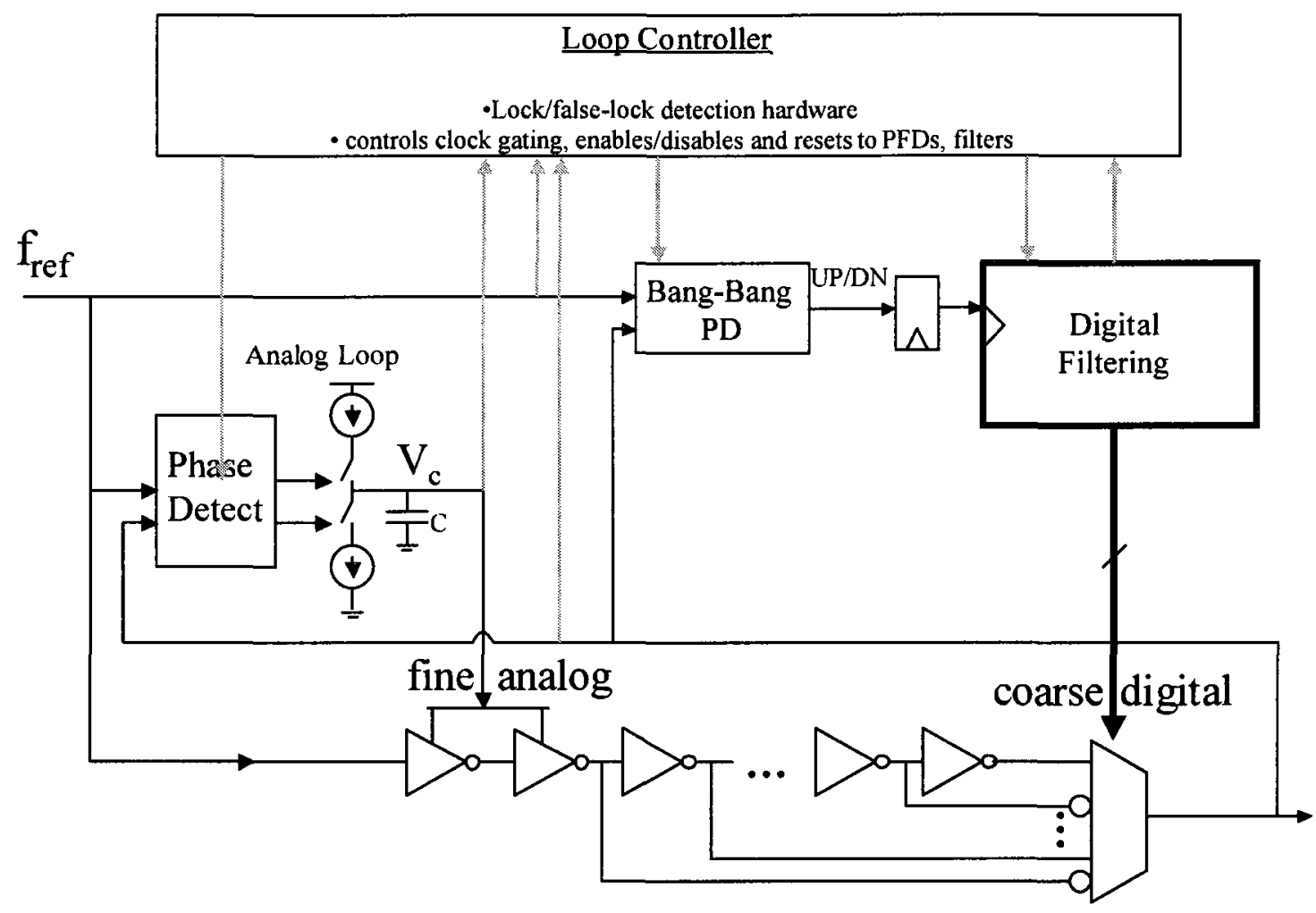

Figure 2.11: Dual-Loop Architecture to reduce analog sensitivity

\subsection{Literature Search}

\subsubsection{Analog Implementations}

Analog DLLs and PLLs make up the majority of implementations. A selection of the relevant literature is presented below, where the focus was on reviewing architectures (or end results) with very low area and low power. One thing to be wary of in reviewing these figures, is that the area of their integrating capacitors, which is typically dominant, is not included in a few of the referenced works. These are indicated by 'active-only' annotations in the table. In general, due to the complexity of the analog biasing arrangements and size of the loop filter, the area and power consumption of analog DLLs or PLLs is typically quite large. 


\begin{tabular}{|c|c|c|c|c|c|c|}
\hline Description & Type & Speed & Tech. & Area & Power & Jitter \\
\hline $\begin{array}{l}\text { Ahn, JSSC, 2000: Compact } 4 \mathrm{x} \\
\text { PLL, 2.5MHz BW, for Ultra- } \\
\text { sparc clock generation uses sin- } \\
\text { gle integrating cap and feedfor- } \\
\text { ward [7]. }\end{array}$ & $\begin{array}{l}\text { Analog } \\
\text { PLL }\end{array}$ & $\begin{array}{lr}8.5 & - \\
660 \mathrm{MHz}\end{array}$ & 0.25 um & $0.09 \mathrm{~mm}^{2}$ & $\begin{array}{l}25 \mathrm{~mW} \\
@ 144 \mathrm{MHz}\end{array}$ & $50 \mathrm{ps}_{p p}$ \\
\hline $\begin{array}{l}\text { Maneatis, ISSCC, 1996: Well } \\
\text { recognized implementation of a } \\
\text { low noise Analog PLL. }[6]\end{array}$ & $\begin{array}{l}\text { Analog } \\
\text { PLL }\end{array}$ & $\begin{array}{l}0.002- \\
550 \mathrm{MHz}\end{array}$ & $0.5 \mathrm{um}$ & $1.91 \mathrm{~mm}^{2}$ & $\begin{array}{l}9.2 \mathrm{~mW} \\
@ 500 \mathrm{MHz}\end{array}$ & $\begin{array}{l}144 \mathrm{ps}_{p p} \\
\mathrm{w} / \mathrm{VDD}- \\
\text { noise } \\
1 \mathrm{MHz} \\
20 \% 12\end{array}$ \\
\hline $\begin{array}{l}\text { Maneatis, ISSCC, 1996: Uses } \\
\text { MDLL approach for clock mul- } \\
\text { tiplication then uses a 2nd DLL } \\
\text { for deskew. }[6]\end{array}$ & $\begin{array}{l}\text { Dual } \\
\text { Analog } \\
\text { DLLs }\end{array}$ & $\begin{array}{l}0.002- \\
400 \mathrm{MHz}\end{array}$ & $0.5 \mathrm{um}$ & $1.18 \mathrm{~mm}^{2}$ & $\begin{array}{l}21 \mathrm{~mW} \\
@ 250 \mathrm{MHz}\end{array}$ & $\begin{array}{l}262 \mathrm{ps}_{p p} \\
\mathrm{w} / \mathrm{VDD}- \\
\text { noise } \\
1 \mathrm{MHz} \\
20 \%\end{array}$ \\
\hline $\begin{array}{l}\text { DaDalt, JSSC, 2003: Low } \\
\text { noise differentially controlled } \\
\text { PLL with active loop filter. }[18]\end{array}$ & $\begin{array}{l}\text { Analog } \\
\text { LC PLL }\end{array}$ & $\begin{array}{l}2.5 \quad- \\
3.1 \mathrm{GHz}\end{array}$ & $0.12 \mathrm{um}$ & $0.7 \mathrm{~mm}^{2}$ & $\begin{array}{l}35 \mathrm{~mW} \\
@ 2.5 \mathrm{GHz}\end{array}$ & $0.86 \mathrm{ps}_{r m s}$ \\
\hline $\begin{array}{l}\text { FarjadRad, JSSC, 2002: Uses a } \\
\text { Multiplying (x4-x10) DLL which } \\
\text { re-seeds a ring-oscillator with } \\
\text { the reference clock each cycle. } \\
{[19]}\end{array}$ & $\begin{array}{l}\text { Analog } \\
\text { Multi- } \\
\text { plying } \\
\text { DLL }\end{array}$ & $\begin{array}{l}0.2 \quad- \\
2.0 \mathrm{GHz}\end{array}$ & $0.18 \mathrm{um}$ & $\begin{array}{l}0.05 \mathrm{~mm}^{2} \\
\text { (Active } \\
\text { only) }\end{array}$ & $\begin{array}{l}12 \mathrm{~mW} \\
@ 2.0 \mathrm{GHz} \\
\text { (includ- } \\
\text { ing } \\
\text { output } \\
\text { buffer) }\end{array}$ & $\begin{array}{l}1.7 \mathrm{ps}_{r m s} \\
13.1 \mathrm{ps}_{p p}\end{array}$ \\
\hline $\begin{array}{l}\text { Cheng, AsiaPacific, 2004: Con- } \\
\text { ventional analog DLL multiplier } \\
\text { with adjustable phase selection } \\
\text { into the edge-combiner. [20] }\end{array}$ & $\begin{array}{l}\text { Analog } \\
\text { DLL } \\
\text { (Simula- } \\
\text { tion) } \\
\end{array}$ & $\begin{array}{l}0.25 \quad- \\
2.2 \mathrm{GHz}\end{array}$ & $0.18 \mathrm{um}$ & $\begin{array}{l}\text { N/A } \\
\text { Simu- } \\
\text { lation } \\
\text { only } \\
\end{array}$ & $\begin{array}{l}6.6 \mathrm{~mW} \\
@ 2 \mathrm{GHz} \\
\text { out } \\
(\mathrm{Sim}) \\
\end{array}$ & $\begin{array}{l}33 \mathrm{ps}_{p p} \\
\text { deter- } \\
\text { ministic } \\
(\mathrm{Sim})\end{array}$ \\
\hline $\begin{array}{l}\text { Kim, JSSC, 2002: Adds ex- } \\
\text { tra logic to phase-detector to } \\
\text { prevent false locks. Otherwise, } \\
\text { a conventional edge-combining } \\
\text { analog DLL with } x 4 \text { multiple. } \\
\text { Delay elements are voltage reg- } \\
\text { ulated CMOS buffers. [21] }\end{array}$ & $\begin{array}{l}\text { Analog } \\
\text { DLL } \\
\text { multi- } \\
\text { plier }\end{array}$ & $1.0 \mathrm{GHz}$ & $0.35 \mathrm{um}$ & $\begin{array}{l}0.07 \mathrm{~mm}^{2} \\
\text { (active } \\
\text { only) }\end{array}$ & $42.9 \mathrm{~mW}$ & $\begin{array}{l}7.28 \mathrm{ps} \\
\text { cycle- } \\
\text { cycle }\end{array}$ \\
\hline
\end{tabular}

\footnotetext{
${ }^{12}$ The high jitter number is a result of this added supply noise $-20 \%$ at $1 \mathrm{MHz}$.
} 


\begin{tabular}{|l|l|l|l|l|l|l|}
\hline $\begin{array}{l}\text { Shi, ESSCIRC, 2006: Small x7 } \\
\text { PLL for integrated LVDS appli- } \\
\text { cations, 1.2MHz BW. } 22]\end{array}$ & $\begin{array}{l}\text { Analog } \\
\text { PLL mul- } \\
\text { tiplier }\end{array}$ & $\begin{array}{l}100- \\
560 \mathrm{MHz}\end{array}$ & $0.35 \mathrm{um}$ & $0.09 \mathrm{~mm}^{2}$ & $12 \mathrm{~mW}$ & $\begin{array}{l}7.1 \mathrm{ps} \\
\text { rms } \\
\text { cycle- } \\
\text { cycle }\end{array}$ \\
\hline $\begin{array}{l}\text { Shi, ESSCIRC, 2006: Small x7 } \\
\text { PLL for integrated LVDS appli- } \\
\text { cations, 1.2MHz BW. [22] }\end{array}$ & $\begin{array}{l}\text { Analog } \\
\text { PLL mul- } \\
\text { tiplier }\end{array}$ & $\begin{array}{l}100- \\
560 \mathrm{MHz}\end{array}$ & $0.35 \mathrm{um}$ & $0.09 \mathrm{~mm}^{2}$ & $12 \mathrm{~mW}$ & $\begin{array}{l}7.1 \mathrm{ps} \\
\text { rms } \\
\text { cycle- } \\
\text { cycle }\end{array}$ \\
\hline $\begin{array}{l}\text { Sai, IEICE, 2008: Low-power, } \\
\text { low-noise clock generator for Rx } \\
\text { chain ADC, 1MHz BW. [23] }\end{array}$ & $\begin{array}{l}\text { Analog } \\
\text { PLL }\end{array}$ & $200 \mathrm{MHz}$ & $0.09 \mathrm{um}$ & $1.1 \mathrm{~mm}^{2}$ & $12 \mathrm{~mW}$ & $\begin{array}{l}3.6 \mathrm{ps} \\
\text { rms long- } \\
\text { term } \\
\text { jitter (es- } \\
\text { timated) }\end{array}$ \\
\hline
\end{tabular}

Table 2.1: Comparison of analog DLL/PLL implementations

\subsubsection{Digital Architectures}

Though the design and integration of digital DLLs/PLLs is much easier than their analog counterparts, because of the digital control, storage, filtering and decoding logic, their area and power inefficiencies are comparable to analog implementations. Meanwhile, because of quantization noise at both the input time-to-digital converter and output $\mathrm{NCO}$, their noise characteristics tend to be far worse.

Table 2.2 compares a number of different all-digital PLLs, and the architectures of three of them are highlighted below.

A digital DLL used for clock deskewing in the Intel Itanium processor, taken directly from Tam [1] is shown in Figure 2.12. In this architecture, a 20-bit delay control register sits inside the local-controller of a deskew buffer. On boot-up, the DLLs are enabled and they align the local clock grids to within 20ps (which is the resolution of the delay element) of the reference clock. In this particular chip, however, Intel made extensive use of intentional skew, and so once the auto-alignment was performed the values inside the delay control register are read, and re-adjusted via a test-access port (TAP) to fine-tune the regional clock grids. In this architecture, because of the coarse tuning, the deskewing elements could not be left on to align 
clocks during operation. Thus, they could only compensate for process variations (to within 20ps) and not for supply, temperature or delay-line noise/fluctuations.

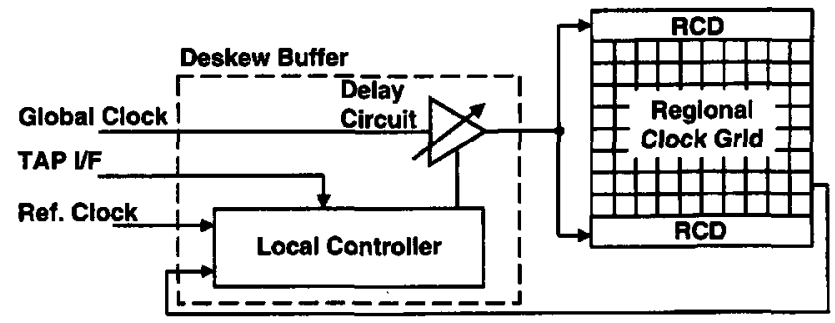

(a) Overview of Active Deskew Architecture, from Tam [1]

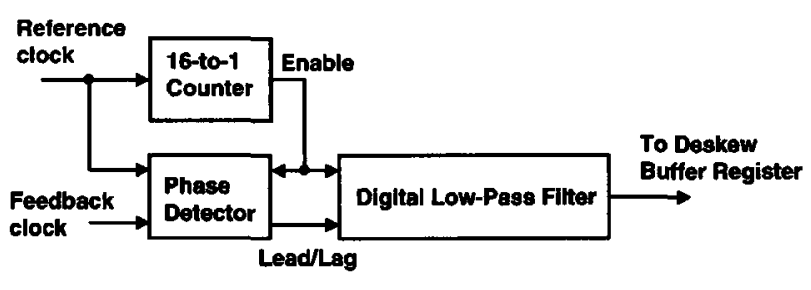

(b) Local Controller, from Tam [1]

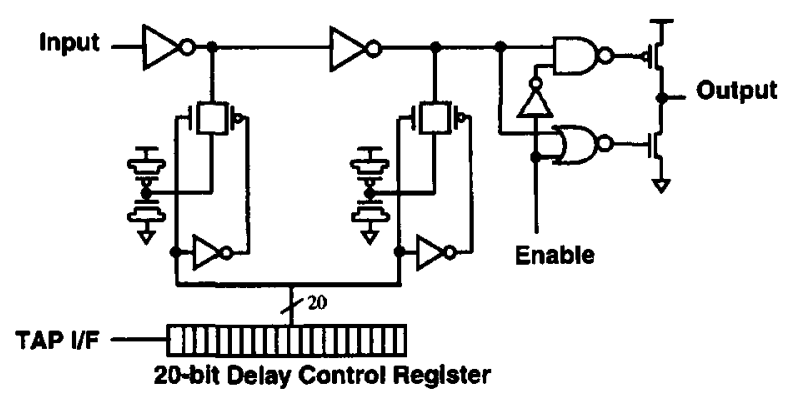

(c) Delay Circuit, from Tam [1]

Figure 2.12: Digital Deskewing DLL as used in Intel Itanium, from Tam [1]

Two different digital PLL implementations are shown in Figures 2.13 and 2.14. Olsson's architecture is quite standard, and is similar to that of the example presented in Figure 2.10. The phase-detector feeds a time to digital converter (T2d). The error signal is sent to a simple recursive filter and applied to a digitally controlled oscillator.

Staszewski's architecture uses an approach similar to the front end of a direct digital synthesizer. That is, he uses a phase accumulator which could otherwise be used to lookup a synthesized waveform. With this approach, the phase information of the reference is always available in this digital phase accumulator, unlike in a conventional PFD where phase information is only available at 0 to 1 and 1 to 0 transitions of the waveform. Similarly, the phase information of the digitally controlled oscillator (DCO) clock is available in the loop's DCO divider. By subtracting these two signals (the phase detector), a digital representation of the phase error is always available. Unfortunately, since there will be some phase error between the DCO clock which 
adjusts the divider and the reference one which adjusts the accumulator, a time-todigital converter (TDC) is still necessary to provide a correction factor. The DCO itself has more than one range of operation. A coarse loop, controlled by the mostsignificant bits out of the digital filter, roughly adjust the capacitance (they use an LC oscillator), and these bits are then fixed. The least-significant bits are decoded into a digital thermometer code and adjust very small varactors in the LC tank. The very small size of the switchable capacitance leads to quantization jitter which is negligible in their application. Though Stasewski's noise results are quite impressive (again they use an LC oscillator), the area and power consumption of his architecture preclude its use in large numbers as contemplated here.

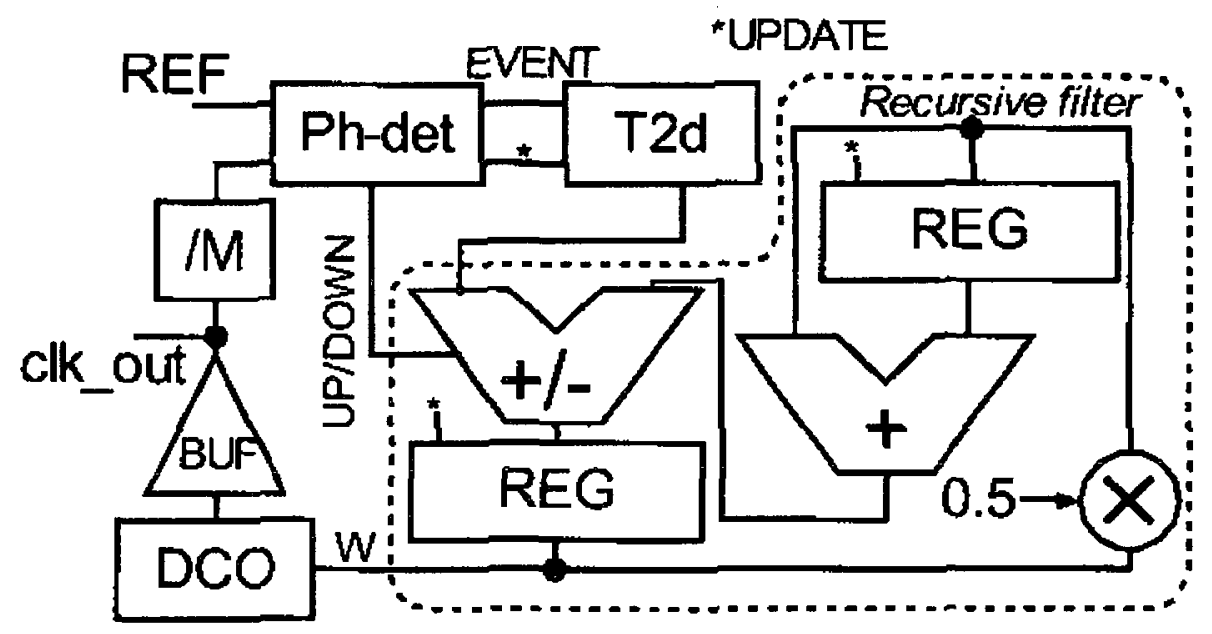

Figure 2.13: Olsson's All-Digital PLL: Standard Implementation [2]

\begin{tabular}{|c|c|c|c|c|c|c|}
\hline Description & Type & Speed & Tech. & Area & Power & Jitter \\
\hline $\begin{array}{l}\text { Olsson, AsiaPac ASIC, 2002: } \\
\text { Time-to-digital based ADPLL. } \\
\text { Shown in Figure 2.13. }[2]\end{array}$ & $\begin{array}{l}\text { Digital } \\
\text { PLL }\end{array}$ & $\begin{array}{ll}152 & - \\
366 \mathrm{MHz}\end{array}$ & $0.35 \mathrm{um}$ & $0.07 \mathrm{~mm}^{2}$ & $\begin{array}{l}\text { N/A: } \\
\text { Com- } \\
\text { ments } \\
\text { that it is } \\
\text { poor }\end{array}$ & $\begin{array}{l}\text { N/A: } 10 \\
-150 \text { ps } \\
\text { resolu- } \\
\text { tion }\end{array}$ \\
\hline
\end{tabular}




\begin{tabular}{|c|c|c|c|c|c|c|}
\hline $\begin{array}{l}\text { Staszewski, JSSC, 2004: Time- } \\
\text { to-digital based ADPLL with } \\
\text { LC DCO and novel phase- } \\
\text { accumulation multiplier. Shown } \\
\text { in Figure } 2.14[3]\end{array}$ & $\begin{array}{l}\text { Digital } \\
\text { PLL }\end{array}$ & $\begin{array}{l}2.4 \\
\mathrm{GHz}\end{array}$ & $0.13 \mathrm{um}$ & $\begin{array}{l}\approx \\
0.6 m^{2} \\
(\text { esti- } \\
\text { mated } \\
\text { from die- } \\
\text { photo) }\end{array}$ & $\begin{array}{l}<37.5 \mathrm{~mW} \\
@ 2.4 \mathrm{GHz}\end{array}$ & $1 \mathrm{ps}_{r m s}$ \\
\hline $\begin{array}{l}\text { Kwak, VLSI, 03: Conventional } \\
\text { digital DLL, in addition to } \\
\text { a secondary digital loop for } \\
\text { duty cycle correction for DDR } \\
\text { SDRAMs. [14] }\end{array}$ & $\begin{array}{l}\text { Digital } \\
\text { Deskew- } \\
\text { ing DLL }\end{array}$ & $\begin{array}{lr}66 & - \\
500 \mathrm{MHz}\end{array}$ & $0.13 \mathrm{um}$ & $\begin{array}{l}>0.1 \mathrm{~mm}^{2} \\
\text { (est. } \\
\text { from die- } \\
\text { photo) }\end{array}$ & $\begin{array}{l}24 \mathrm{~mW} \\
@ 400 \mathrm{MHz} \\
60 \mathrm{~mW} \\
@ 500 \mathrm{MHz}\end{array}$ & $25 \mathrm{ps}_{p p}$ \\
\hline $\begin{array}{l}\text { Fahim, ESSCIRC, 2003: } \\
\text { Super-sampling conventional } \\
\text { ADPLL.[15] }\end{array}$ & $\begin{array}{l}\text { Digital } \\
\text { PLL }\end{array}$ & \begin{tabular}{l|}
$30 \quad-$ \\
$160 \mathrm{MHz}$
\end{tabular} & $0.25 \mathrm{um}$ & $0.31 \mathrm{~mm}^{2}$ & $\begin{array}{l}3.12 \mathrm{~mW} \\
@ 144 \mathrm{MHz}\end{array}$ & $\begin{array}{l}60 \mathrm{ps}_{r m s} \\
130 \mathrm{ps} \\
\text { cycle-cycle }\end{array}$ \\
\hline $\begin{array}{l}\text { Chung, JSSC, 20003: All digital } \\
\text { standard cell PLL. [24] }\end{array}$ & $\begin{array}{l}\text { Digital } \\
\text { PLL }\end{array}$ & 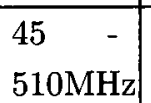 & $0.35 \mathrm{um}$ & $0.71 \mathrm{~mm}^{2}$ & $\begin{array}{l}100 \mathrm{~mW} \\
@ 500 \mathrm{MHz}\end{array}$ & $70 \mathrm{ps}_{p p}$ \\
\hline
\end{tabular}

Table 2.2: Comparison of digital DLL/PLL implementations

\subsubsection{Mixed-Signal Architectures}

Though the mixed-mode dual-loop approach can offer reduced noise sensitivity, it comes at a significant cost in terms of area and power consumption to support the second control loop, and to perform the necessary switching between the two.

\begin{tabular}{|c|c|c|c|c|c|c|}
\hline Description & Type & Speed & Tech. & Area & Power & Jitter \\
\hline $\begin{array}{l}\text { Kim, JSSC, 2000: Mixed digi- } \\
\text { tal outer loop, low-gain analog } \\
\text { inner loop DLL for wide range } \\
\text { deskewing in SDRAMs [25] }\end{array}$ & $\begin{array}{l}\text { Mixed- } \\
\text { Mode } \\
\text { DLL }\end{array}$ & $200 \mathrm{MHz}$ & $0.6 \mathrm{um}$ & $0.45 \mathrm{~mm}^{2}$ & $\begin{array}{l}33 \mathrm{~mW} @ \\
200 \mathrm{MHz}\end{array}$ & $\begin{array}{l}6.38 \mathrm{ps}_{r m s} \\
49 \mathrm{ps}_{p p}\end{array}$ \\
\hline $\begin{array}{l}\text { Maxim, JSSC, 2005: Low noise } \\
\text { analog PLL to generate } 8 \text { refer- } \\
\text { ence phases then distributes to } \\
\text { digitally controlled analog inter- } \\
\text { polators to control phase shift in } \\
\text { a deskew application. [26] }\end{array}$ & $\begin{array}{l}\text { Analog } \\
\text { PLL + } \\
\text { Digital } \\
\text { Interpo- } \\
\text { lator }\end{array}$ & $\begin{array}{l}0.2 \\
\leftrightarrow \quad 2.5 \\
\mathrm{GHz}\end{array}$ & $0.16 \mathrm{um}$ & $0.32 \mathrm{~mm}^{2}$ & $60 \mathrm{~mW}$ & $32 \mathrm{ps}_{p p}$ \\
\hline
\end{tabular}




\begin{tabular}{|c|c|c|c|c|c|c|}
\hline $\begin{array}{l}\text { Bae, JSSC, 2005: Uses a con- } \\
\text { ventional analog DLL to gener- } \\
\text { ate reference phases and coarse } \\
\text { digital logic to send one of these } \\
\text { phases into a secondary analog } \\
\text { DLL. If the phase selection is } \\
\text { properly controlled then it can } \\
\text { track an infinite phase shift. [27] }\end{array}$ & $\begin{array}{l}\text { Mixed } \\
\text { Mode } \\
\text { Deskew } \\
\text { DLL }\end{array}$ & $\begin{array}{l}60 \\
760 \\
\mathrm{MHz}\end{array}$ & $0.18 \mathrm{um}$ & $\begin{array}{l}0.19 \mathrm{~mm}^{2} \\
\text { (Active } \\
\text { only) }\end{array}$ & $\begin{array}{l}63 \mathrm{~mW} \\
@ 700 \mathrm{MHz}\end{array}$ & $60 \mathrm{ps}_{p p}$ \\
\hline
\end{tabular}

Table 2.3: Comparison of mixed-mode DLL/PLL implementations 


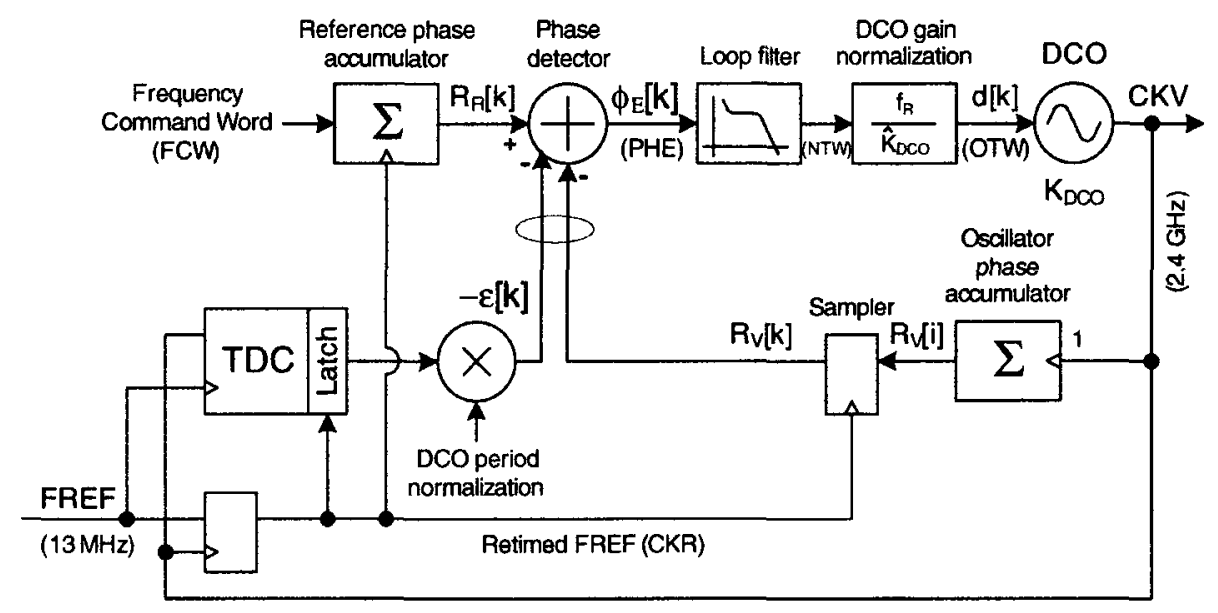

Figure 2.14: Staszewski's All-Digital PLL: Very-low phase-noise, high complexity [3] 


\section{Chapter 3}

\section{Cascaded Charge-Pump: A System Level Perspective}

\subsection{Overview}

Both analog and digital implementations of PLLs and DLLs are too large for extensive use as clock control and deskewing elements inside ICs. With advancing technology and reducing voltage swing, analog implementations are forced to increase VCO sensitivity, which forces larger filter sizes and reduces performance. Digital architectures are plagued by quantization effects and often larger control and filter structures. Dualloop approaches can reduce VCO gain so that the loop-filter is smaller, but they have difficulty maintaining lock across temperature changes, and suffer from the increased complexity, and lock-time of a two-pronged approach. Keeping in mind that the main goal is for very small PLLs and DLLs, the cascaded charge-pump circuit introduced here must be very simple and area efficient.

The cascaded charge-pump, introduced in Figure 3.1, is primarily an analog integrator, but it produces a set of $\mathrm{N}$ output control voltages to modulate the VCO or delay line. In normal operation, the cascaded charge-pump is working on only a single control node at once, and the situation and loop-dynamics exactly mirror the case of a conventional analog PLL with a reduced VCO gain. If the voltage on the control node begins to saturate, the cascaded charge-pump starts to exercise the neighbouring control. Using this approach repetitively, the control range can be extended indefinitely.

The VCO is modulated by an N-stage set of controls, but the cascaded chargepump only exercises a couple of these elements at a time. Because the control is 
spread amongst a number of stages, the sensitivity of the VCO to any individual node is reduced by a factor of $\mathrm{N}$. This effective reduction in VCO gain can be used to directly reduce filter requirements and therefore circuit area, or more productively, it can be traded for increased charge-pump gain and thus better synthesizer noise performance. With better synthesizer performance relative to the VCO, the optimal loop-BW for minimal system noise moves further out and this, in turn, will result in smaller filters.

\section{Custom Simulators}

Two system level PLL simulators have been written to characterize various aspects of PLL behaviour. The second, and more elaborate of the simulators, runs 20,000x faster than transistor level simulations and 300x faster than behavioural Verilog-A models. It can take in approximately 40 different loop parameters on the fly and has a numerical noise floor better than $-200 \mathrm{dBc} / \mathrm{Hz}$ with a $50 \mathrm{MHz}$ reference. The simulator allows the closed-loop analysis of non-linear effects into the $\mathrm{kHz}$ resolution with only a few seconds of simulation time. The simulator will be used to confirm that the cascaded charge-pump does indeed behave as a low-gain analog PLL and has the associated benefits of low filter sizes and better noise immunity.

\subsection{Cascaded Charge-Pump Simplified}

Figure 3.1 shows the use of the new cascaded charge-pump (CCP) inside the control loop of a PLL. Whereas analog loops use a single control voltage to regulate the VCO, this approach uses an $\mathrm{N}$-signal vector $(N=6$ in the example). Logic restrains most of the control vector at 1 or 0 (VDD or VSS), and steers the analog charge-pump current and loop-filter to a single 'active' analog node (shown at $V c_{4}$ in this example).

Assume, for the moment, that an application demanded a VCO range of $100 \pm 30 \mathrm{MHz}$. In a single voltage system with $1 \mathrm{~V}$ of available swing, this would necessitate a VCO gain of $60 \mathrm{MHz} / \mathrm{V}$. By implementing the VCO control with a 6signal vector, the gain/signal can be reduced to $10 \mathrm{MHz} / \mathrm{V}$, while still satisfying the application requirements. More generally, given equivalence of other parameters, the vectored system would behave identically to an analog one with VCO gain $K_{V} / N$. 


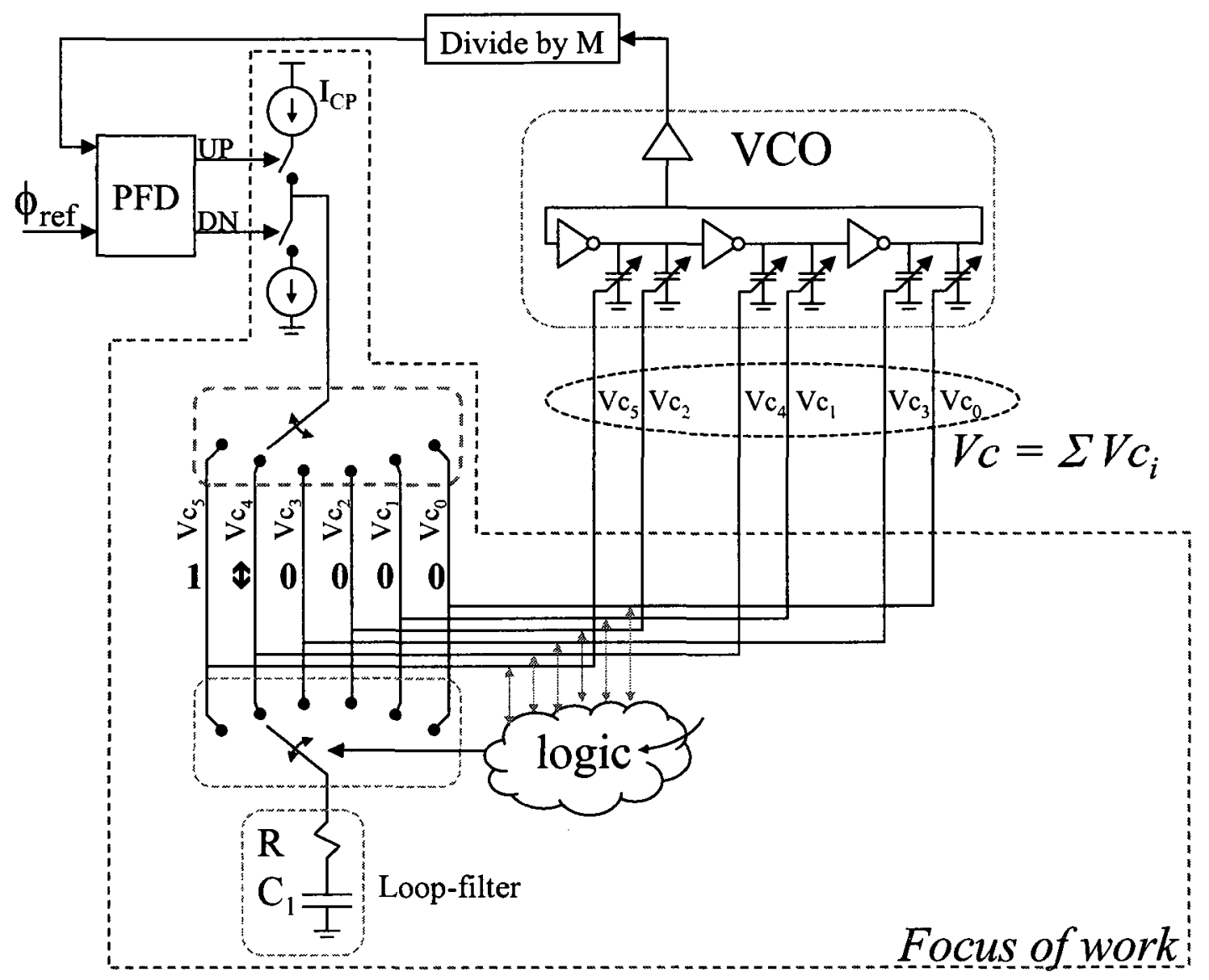

Figure 3.1: Cascaded Charge-Pump Architecture: A vector of signals regulate the VCO. Analog control is steered to a single node, while digital logic holds the others at VDD $(\operatorname{logic} 1)$ or VSS $(\operatorname{logic} 0)$. Any individual node has only a minor effect on the VCO frequency, and so this reduces the system's sensitivity to the analog voltage and its associated noise. The effective reduction in $K_{V}$ is used to reduce filter size and improve noise suppression without sacrificing output range.

As described in Section 2.6.2, this effective reduction in $K_{V}$ can be used to reduce capacitance requirements and thus die-area, and/or it can be used to reduce in-band noise, which permits increased bandwidths that also lower filter size. It will also be shown how a simple tri-state delay-line forms the core of the system to regulate and steer the analog control to an appropriate node. Designed for standardcell compatibility and automated placement and routing, the inherent HW simplicity 
makes the architecture attractive compared to conventional analog, digital or mixedsignal solutions.

\subsection{Current Steering for Vectored Control}

Figure 3.1 shows a charge-pump controlled by a conventional phase-frequency detector. The CCP generates a thermometer coded vector at the output - that is, a set of 1 's followed by the analog transition region, then a set of $0 \mathrm{~s}$. The $\pm I_{C P}$ out of the charge-pump is steered to the 'analog' node at the transition point of the code-word. For example, if the control word were $1 \uparrow 0000$, the $\uparrow$ represents the node which should fall under analog control and take on a steady-state voltage between logic 0 and 1 . In Figure 3.1, this corresponds to node $V c_{4}$. DN commands from the PFD sink current away from $V c_{4}$, whereas UP commands turn on the current-source and charge $V c_{4}$ toward 1.

\subsubsection{Current-Steering in the Cascaded Charge Pump}

The circuit responsible for directing current flow from the charge-pump to the appropriate node could be implemented in a number of ways. One approach, which is particularly simple from an implementation perspective, is to combine the functions of the charge-pump and the current-steering switch into a delay-line structure.

Figure 3.2c illustrates how a charge-pump can be built with digital tri-state buffers. Fundamentally, both the charge-pump and tri-state gate deliver current while enabled, and are high-impedance otherwise. While asserted, UP or DN control signals are pulse-width modulated by a phase-detector, and in turn they force charge into or out-of the load. A load capacitor integrates the charge to form a variable analog voltage. The disadvantage of the 'digital gate' charge-pump is that its current varies more significantly with output voltage than a conventional pump. This is a concern when linearity is paramount (as in fractional synthesizers), but is often not critical in other applications. In Figure 3.2d, one can see the start of a cascade forming. During UP pulses, the top buffer drives the load to 1 , and during DN pulses the bottom gate 


\section{Creating a cascaded charge-pump}

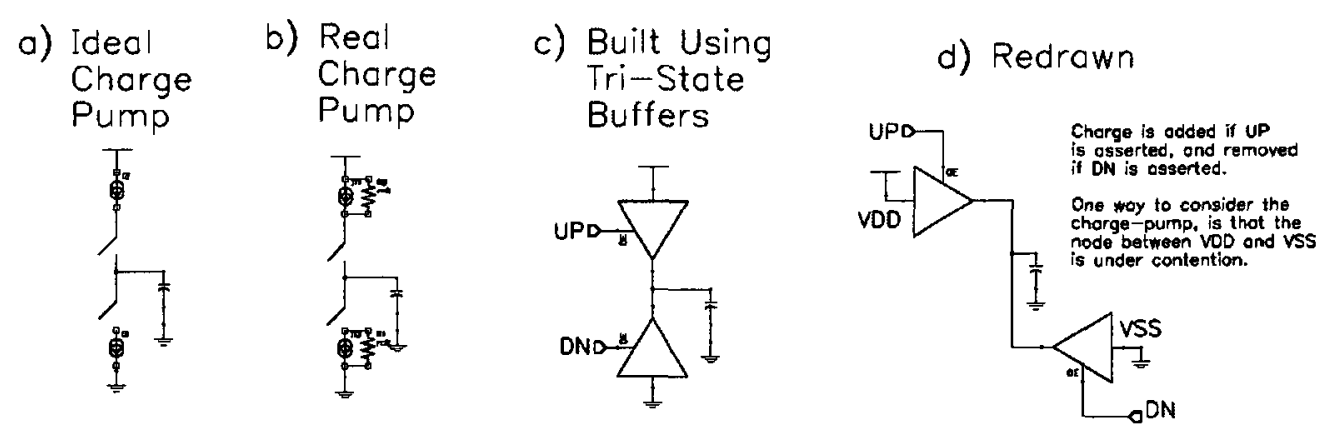

e) Added a 'dummy' tri-state.

f) A 2-stage charge-pump
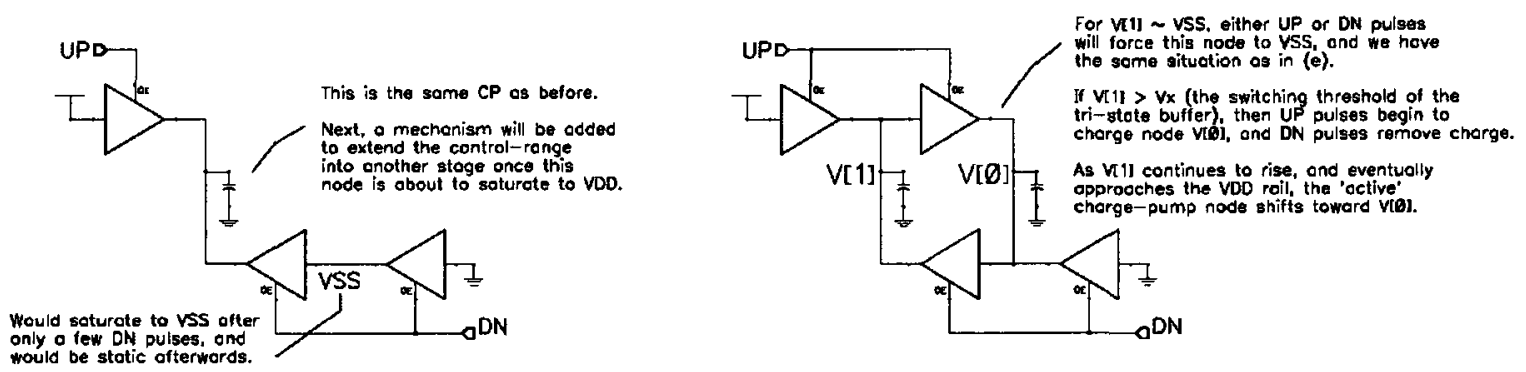

Figure 3.2: An analog charge-pump is shown here being constructed with standard digital tri-state buffers. In the final stages, a cascade is formed such that when one output node saturates, the next begins to take on the task.

pulls the node to $0{ }^{1}$ When the node gets close to a voltage rail, it can be used to enable the next stage of the pump, as shown in panel $f$.

Four stages are shown in a cascade in Figure 3.3. Two chains of tri-state buffers are coupled together in opposite directions. Assume for the moment that the UP and DN signals are mutually exclusive, and that each node (with its associated output capacitance) is initially discharged (ie. $V c_{[3: 0]}=0000$ ). While an UP or DN input from the phase-detector is asserted, it enables either the bottom or top delay-line ${ }^{2}$. If the DN signal is asserted, it enables the top delay-line which begins charging $V c_{3}$ toward 1. As the control voltage slowly charges, it modulates a varactor of the delay line, exposes more capacitance and slowing it down. If the DN signal is left asserted long enough for $V c_{3}$ to charge past the switching threshold of the next gate, $V c_{2}$

\footnotetext{
${ }^{1}$ The issue of current mismatch is addressed in Chapter 4.

${ }^{2}$ It will be shown that tri-state inverters can be used instead, and that even these can be simplified.
} 
Corection pulse from phase-detector

- widh is proporional to phase-error

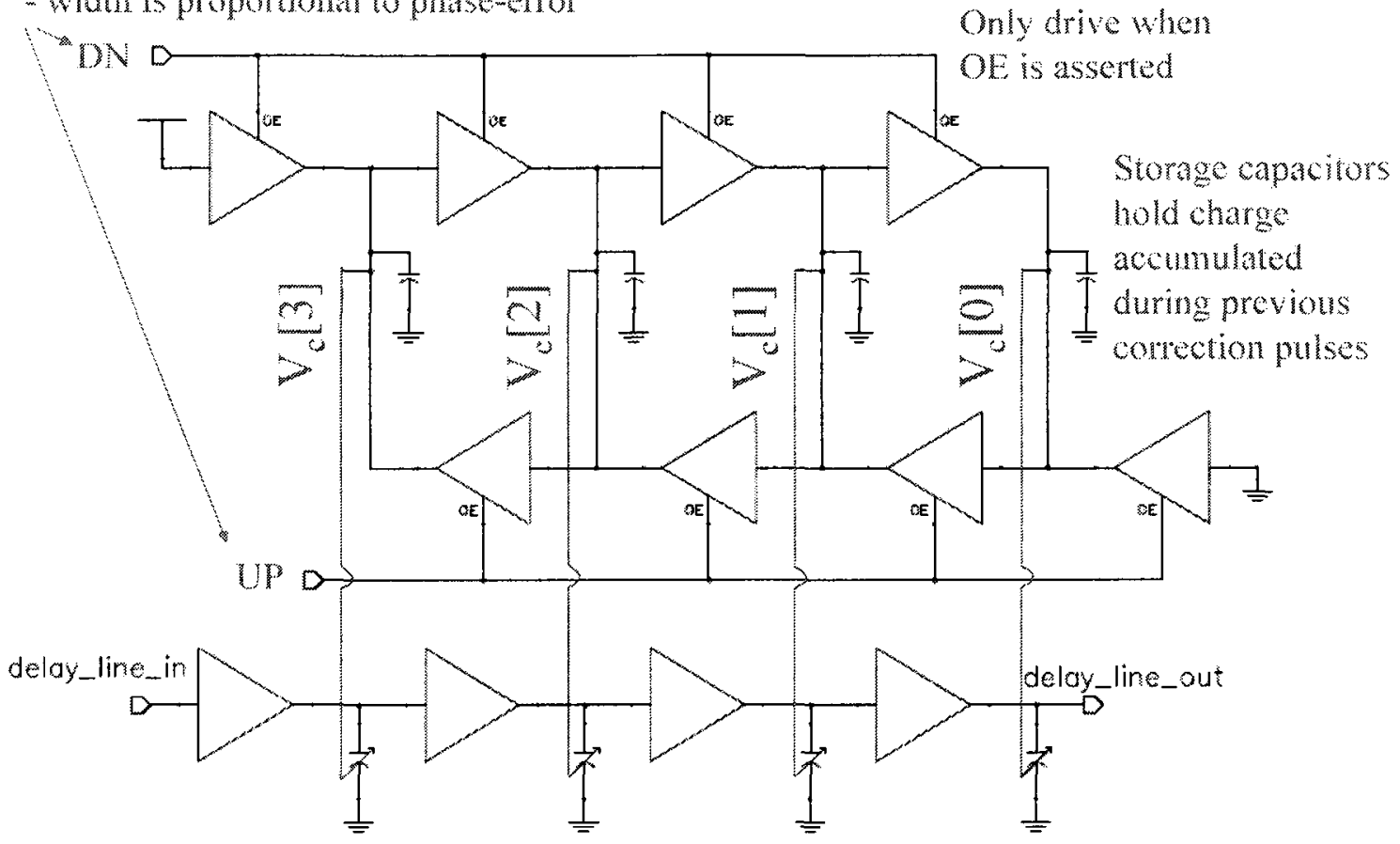

Control ners Vo[3:0 are used to adjust a delay-line (in a DLL), or VOO (in a PLL)

- an exanyle of such a controlled delay-line is shown here

Figure 3.3: A four stage cascaded charge-pump is shown here which would be suitable for DLL operation. DN control signals drive 1's toward the right, raising the varactor voltages and slowing down the delay-line, whereas UP signals drive 0's toward the left, successively discharging control-voltages and removing capacitance from the delayline. In steady-state the control nodes will settle to a value such as $1 \uparrow 00$, where $\uparrow$ represents the node undergoing analog integration from the pumps.

will start to charge, followed eventually by $V c_{1}$, etc... in succession from left-toright. When the control signal is released, any node which is driven only partially toward either voltage rail will hold that analog level ${ }^{3}$. It is this analog refinement of the control vector which sets the new method of this thesis apart from digital implementations used elsewhere [3] [2]. If the DN signal is left asserted, then the control string would eventually saturate to all ones (ie. 1111), which is the limit of the control range. Similarly, if only the UP signal (and hence the lower chain is enabled), it discharges the nodes in succession from right-to-left toward 0.

\footnotetext{
${ }^{3}$ subject to leakage constraints
} 
Taken together, the UP and DN control signals coupled into this dual-direction delay-line cause a thermometer coded analog vector (eg. 1111111 $₫ 00000$, for $\mathrm{N}=13$ ) to slowly shift toward the right (during slow-DN pulses) or left (during speed-UP pulses). This analog shifting forces more charge into or out-of the node at the transition point of the code. At lock, both UP and DN pulses are typically on for a very short time, and the two delay lines are competing in the intermediate cell. At that position, the charge is integrated, as in a conventional charge-pump/loop-filter, to produce a stable analog control voltage. If during the integration process, the node approaches its digital limit, seamlessly the next position in the code begins to fall subject to PFD control and the integration task is gracefully handed down the line.

\subsubsection{Transition between control nodes}

As in a conventional charge-pump, repeated UP commands, for example, will cause $V c_{3}$ to saturate toward VDD. In the cascaded charge-pump, however, node $V c_{2}$ will start to become exercised, picking up the slack as $V c_{3}$ falls out of service. It is important to evaluate how graceful the hand-off is as one control voltage saturates and the next is switched under analog control. To maintain the thermometer coded characteristic, the charge-pump in/out current should now be steered away from $V c_{3}$ to $V c_{2}$, which would begin to charge or discharge as appropriate. From a system level perspective, if the total charge introduced or removed from the system for a given $\mathrm{UP} / \mathrm{DN}$ pulse remains consistent, then it is not critical whether the charge is actually integrated on $V c_{3}, V c_{2}$, or in some combination.

This permits 'soft-handoff' of the charge-pump current and simplifies the constraints on the analog steering logic. During this soft hand-off process (as the analog control moves from one node to its neighbour), the total current out of the charge pump should remain constant, but it may be unequally distributed and cause both the 'outgoing' node (eg. the signal saturating toward 1) and the 'incoming' node (its neighbour which is starting to charge from 0 ) to exhibit analog levels simultaneously. This behaviour is illustrated in Figure 3.4. Since both nodes are still changing dynamically under control of the analog loop, they must both be filtered. This can be done by connecting a filtering load to each output, or more intelligently by switching 
filter sections to the active analog node(s). More information on how the filters are multiplexed is presented in Section 4.6.

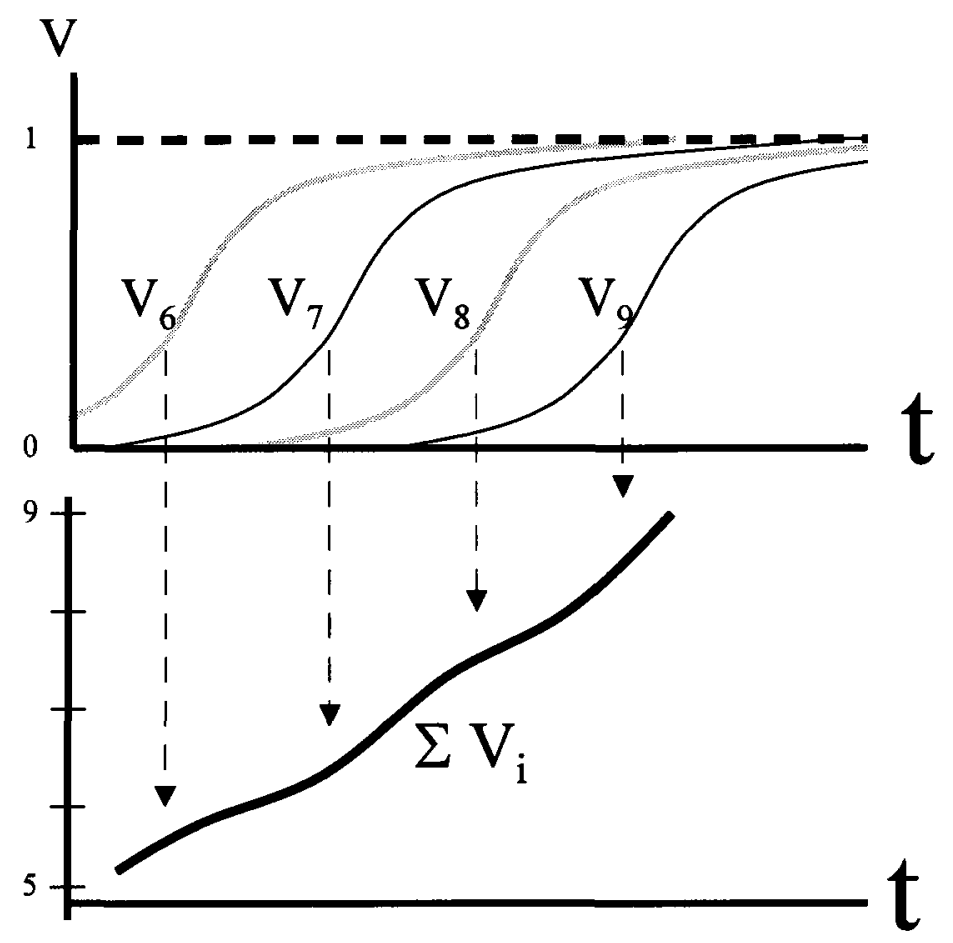

Figure 3.4: Soft Handoff of Control Nodes: As one node saturates toward a voltage rail, the next is enabled. The conglomerate control voltage can be controlled such that it is approximately linear, and is certainly monotonic.

\subsubsection{Example of Locking a DLL with a Cascaded Charge- Pump}

A complete example of a DLL using the cascaded pump, along with simulation results, is shown in Figure 3.5. The top-panel shows a simplified schematic. ${ }^{4}$ The parasitic capacitance of the varactor control input was used to hold the charge distributed by the cascaded pump and an explicit control-storage capacitor is omitted. The reference

\footnotetext{
${ }^{4}$ The simulation was actually performed with intermediate inverting stages in the thermometer code (to be discussed in Section 4.2.1), and with intermediate driver stages in the delay-line (not shown).
} 


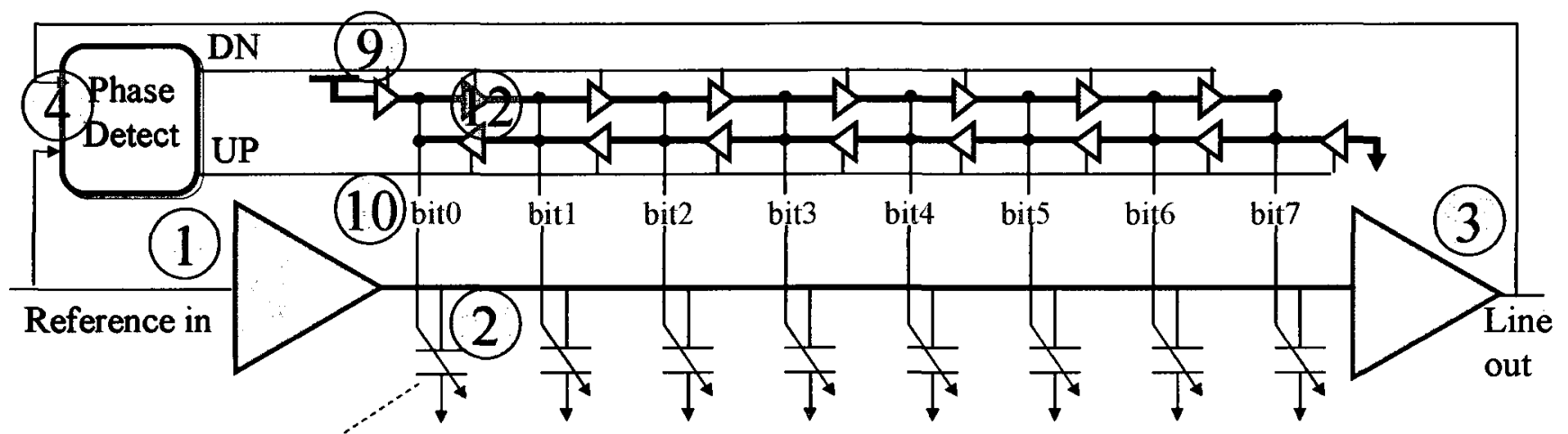
varactor

$$
+
$$

More capacitance slows line down.

Delay tunes to one reference period.

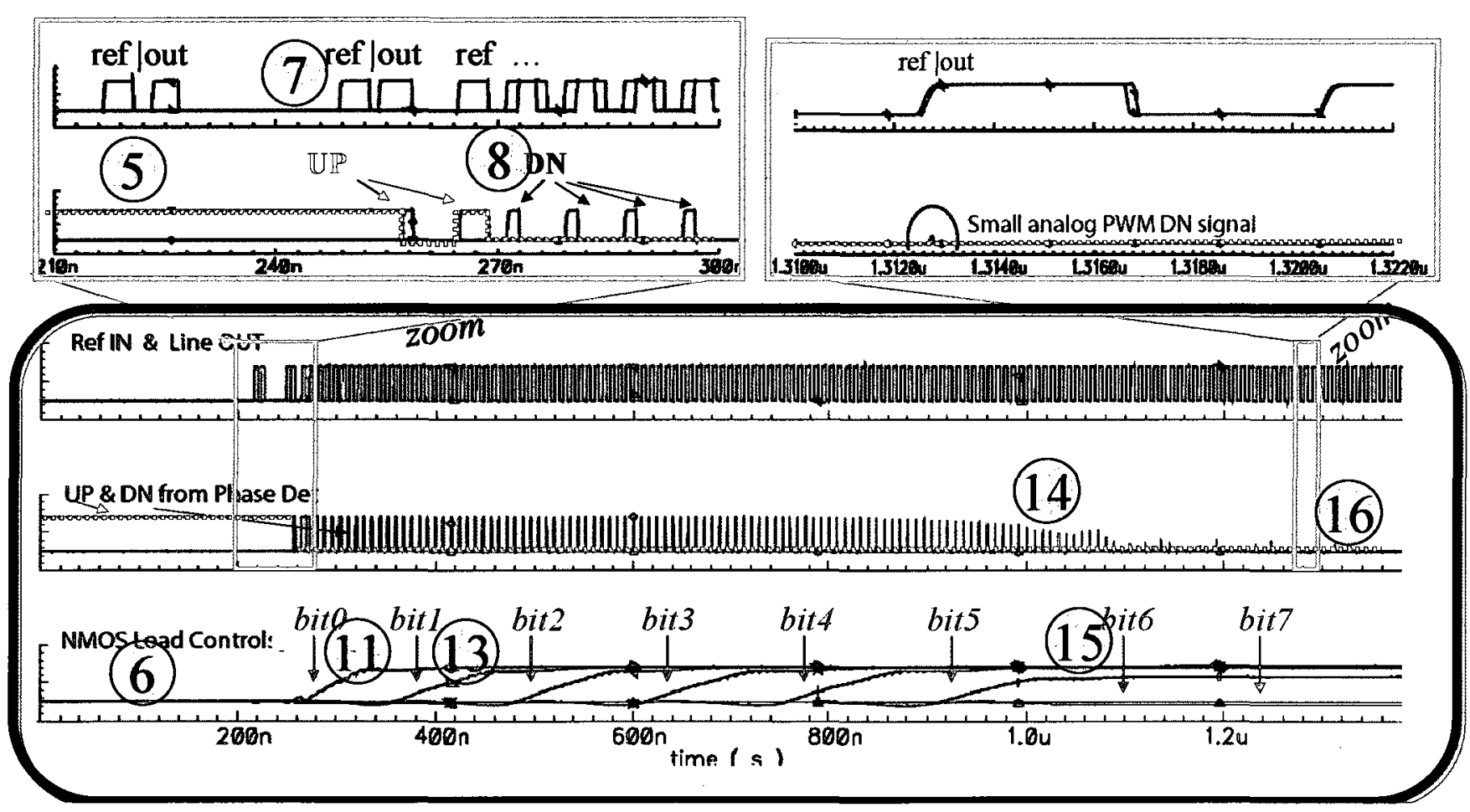

Figure 3.5: Simulation results of a Cascaded charge-pump filter used in a DLL configuration 
clock enters the delay line at (1). The delay-line is modulated by a set of varactor loads (2), which are controlled by the CCP. When the signal emerges from the delay-line (3), its phase is compared to the reference-input at the phase detector (4). During the initial stages of the simulation (5), the phase detector is held in reset, which happens to hold the speed-UP signal asserted. This ensures that the load controls (6) begin in the discharged state and the delay-line is in its fastest configuration (they could instead have been initialized in the all-ones/slowest condition). In this initial stage of the simulation, the test-bench sends only single reference pulses through the delay-line in order to clearly see the delay from input to output $(\approx 7 \mathrm{~ns})$. At $(7)$, it can be seen that the delay in this state is only slightly longer than a half reference period from input to output. With reset released and the reference turned on, the loop begins to operate. At (8), since the delay-line is too fast, the line-out arrives too early relative to the next reference edge, and the slow-DN signal is asserted. While DN is asserted, the tri-state driver at (9) starts to charge the bit $0^{5}$ control node $(10),(11)$, in short bursts, exposing more capacitance to the line and slowing it down. Once bit0 is above the switching threshold of the next stage driver (12), it begins to charge the bit1 node (13). The process continues, successively charging more nodes and slowing down the line, and bringing the line-out and reference signals close enough that the DN pulses from the phase-detector no longer even reach full-rail(14). The progressively skinny pulses, and then even those which don't quite make it to full rail, continue to charge the control nodes (at a progressively slower rate) until eventually dead-zone limits of the phase-detector or charge-pump are reached $(\approx 40 \mathrm{ps}$ in this example). At this point the signals are in-phase, and only very-small UP or DN signals from the phase detector are issued (16).

\subsubsection{Use in PLLs vs DLLs}

Depending on whether the filter structure is to be used in a DLL or PLL, a different loading configuration is required on the output of each charge-pump node. A conceptual diagram of the two approaches is shown in Figure 3.6. The distinction is required to insert a stabilizing zero into the filter transfer function $F(s)$ of the PLL, as mentioned in Chapter 2. While these diagrams show loading filters on each node

\footnotetext{
${ }^{5}$ bit is actuall a misnomer here, since the node can take on a steady-state analog voltage and the term 'bit' may imply digital only operation.
} 


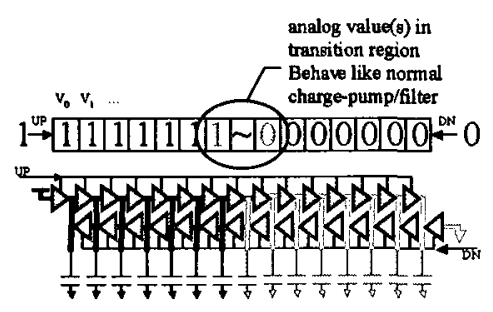

(a) For DLLs, and Type I PLLs

Pure Integrator or low-pass filter

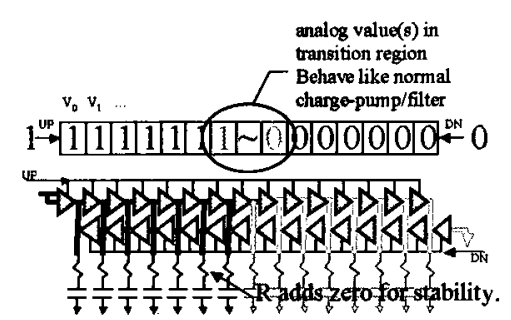

(b) For Type II PLLs Adds $\omega_{z} @ 1 / R C$

Figure 3.6: Depending on whether the cascaded charge-pump is intended for use in a PLL or DLL, the loading circuit is a simple capacitor or an RC filter.

of the filter, in practice only a few filtering loads are used and are multiplexed to the necessary analog nodes.

\subsection{Conventional vs a Cascaded Charge-Pump Con- trolled PLL}

To quickly characterize the system under different scenarios, system level mixedsignal models were developed in behavioural Verilog and then in Verilog-A with first order transistor models. Finally full Spectre simulations were performed on subsets of the entire circuit. As mentioned, the first-order analysis of the presented structure mirrors that of a conventional analog PLL with VCO gain $K_{V} / \mathrm{N}$.

To illustrate, the test-bench shown in Figure 3.7 simulates a conventional ana$\log$ PLL with a low $K_{V}\left(K_{V} / 10\right)$ in comparison to a 10-node control system. In the multi-node system, each node is loaded by $1 / 10^{\text {th }}$ the capacitance, such that the total storage capacity in both simulations is equivalent. Furthermore, the multi-node architecture is modeled with a $20 \%$ variation in $I_{C P}$ as the transition point of the code is handed-off between nodes.

The transient response of both a single control-voltage PLL with $K_{V} / 10$ and the 10-node system is shown in Figure 3.8.

The control-vector is initialized to all zeros. As the acquisition process proceeds, UP signals from the PFD are repetitively asserted and cause the control voltages to successively charge. The control vector overshoots through the proper lock 


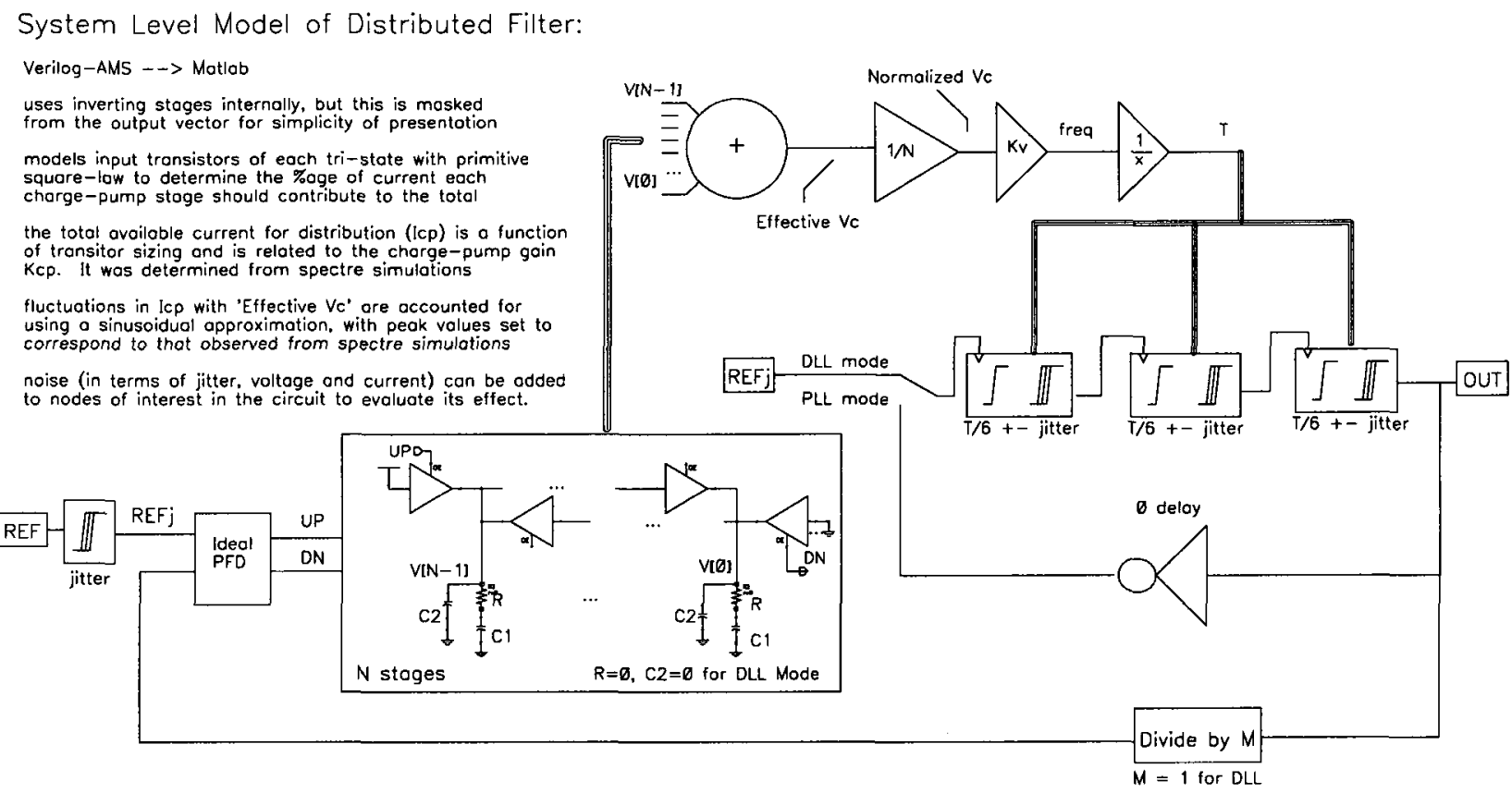

Figure 3.7: An early system-level testbed was used to model the closed-loop transient behaviour of the architecture. The model uses first order transistor approximations along with simulated Spectre data to distribute charge into the various loads as a function of the various voltages.

level and DN signals pull the system back down into alignment. The sum of the control vector, $V_{\text {effective }}$, follows the expected response of a damped second order system.

Of particular relevance, the control signals match between the conventional analog scenario with a low VCO gain, and in the presented architecture (with 10x larger VCO control swing). ${ }^{6}$ While the equivalence of the dynamic response is apparent, but there are two critical differences:

\section{Control Range}

In the single node case, Figure $3.8 \mathrm{a}$, the control voltage is limited to $1 \mathrm{~V}$ due to supply restrictions. In the multi-bit system the control is a conglomerate of 10 individual voltages, and effectively ranges from 0 to $10 \mathrm{~V}$. This has two important advantages: 1) the multi-node system range can be extended without running

\footnotetext{
${ }^{6}$ There is a slight variation between the two cases which is caused entirely by the modeled $I_{C P}$ variation as the thermometer code's transition point is swept.
} 
(a)

Normal

Single voltage

PLL Locking
$N=1: V_{c}$ for normal CP/Loop-filter uses $R_{1}=10 \mathrm{kOhm}, C_{1}=42 \mathrm{pF}, C_{2}=400 f F$

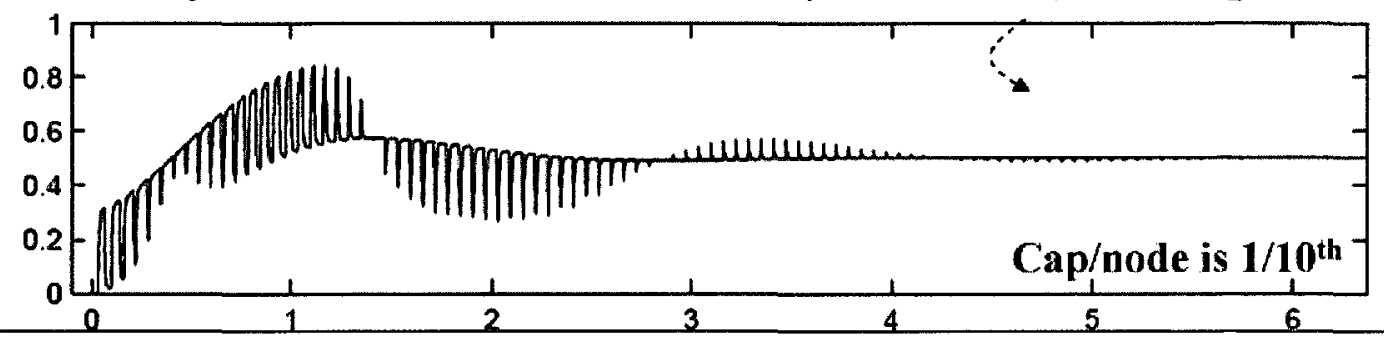

$\times 10^{-6}$

(b)

$\mathrm{N}=10: V_{\text {effective }}=\Sigma V_{i}, V_{i} i=10 . .1, R_{1}=100 \mathrm{KOhm}, 10 \times C_{1}{ }^{\prime}=4.2 p F, C_{2}=40 \mathrm{fF}$

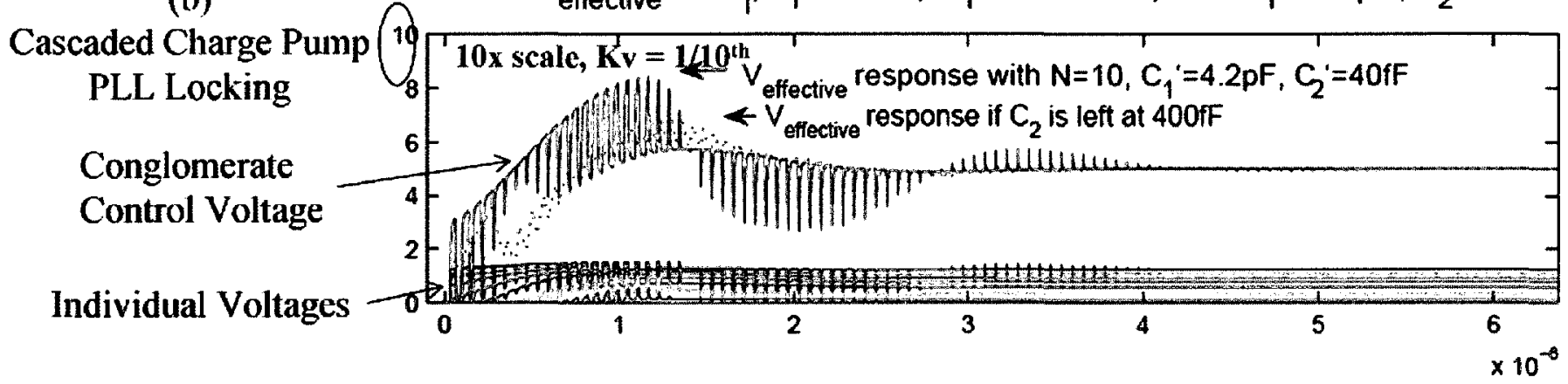

Figure 3.8: Equivalence of Low Gain Analog PLL and Cascaded Pump PLL: Transient simulations of the system level model show the acquisition stage of both a normal analog loop and the cascaded charge-pump structure. Note that the responses match, with the notable exceptions that the effective control range of the cascaded chargepump is from 0 to 10 , and the natural loop is only 0 to 1 . Also of note, the capacitance required per node of the thermometer structure is $1 / \mathrm{N}$ the requirements of a typical analog filter. Note however, that only 2 to 3 of the nodes in the filter are ever changing at a time, and so the we will be able to share a small number of these smaller capacitors among the entire group for significant area savings.

into voltage headroom limits, and 2) the system is naturally less sensitive to any voltage variations/noise on the control line.

2. Capacitance/Area reduction

Though the total capacitance in the two simulations is the same, in the case of the multi-node structure it is distributed across each individual control. In operation, only 2 to 3 nodes are under analog manipulation at a time, and the other capacitors are unnecessary. This opens up the possibility for dynamic sharing of the filter structure. For the case of a 60 stage cascaded chargepump, only $3 \mathrm{RC}$ filter structures are circulated around the pump and a $20 \mathrm{x}$ 
reduction of the passive components (typically the dominant area cost in a PLL) is achieved.

\subsubsection{Effect of non-linear current on Acquisition}

To further examine the effects of the non-linear $I_{C P}$ variation of the non-ideal pumps. Figure 3.9 illustrates a 10 stage cascaded charge-pump locking under ideal conditions, as well as in the presence of a 50\% current fluctuation caused by the imperfect handoff between analog control positions. These simulations show no significant effects on acquisition, even for current deviations much larger than that predicted by extracted Spectre simulations (to be shown in Chapter 4).

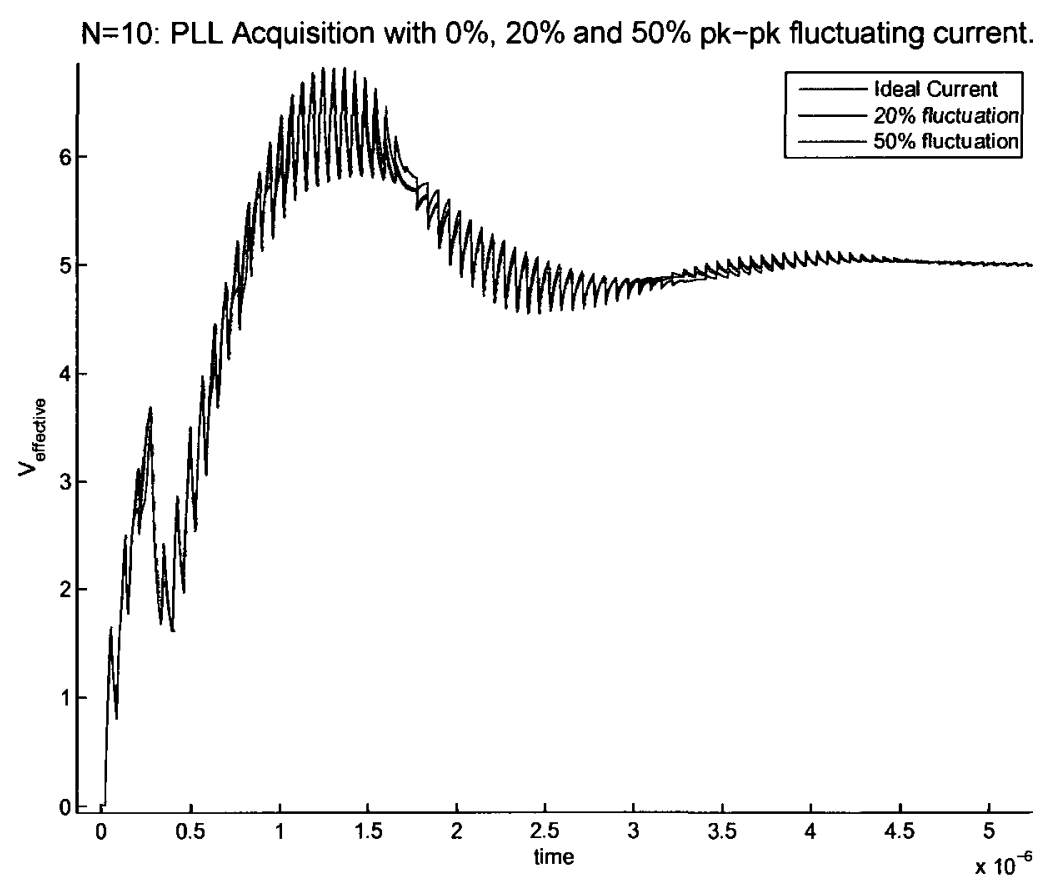

Figure 3.9: System levels simulations were performed to verify that the variable current source/sink capability of the non-ideal charge-pumps did not effect system stability. Spectre simulations show only $12 \%$ variation, and this tests illustrates no delerious effects even with $50 \%$ current variation duration analog handoff from one node to another. 


\subsection{Benefits of Reduced VCO Gain}

\subsubsection{Improved Noise Suppression}

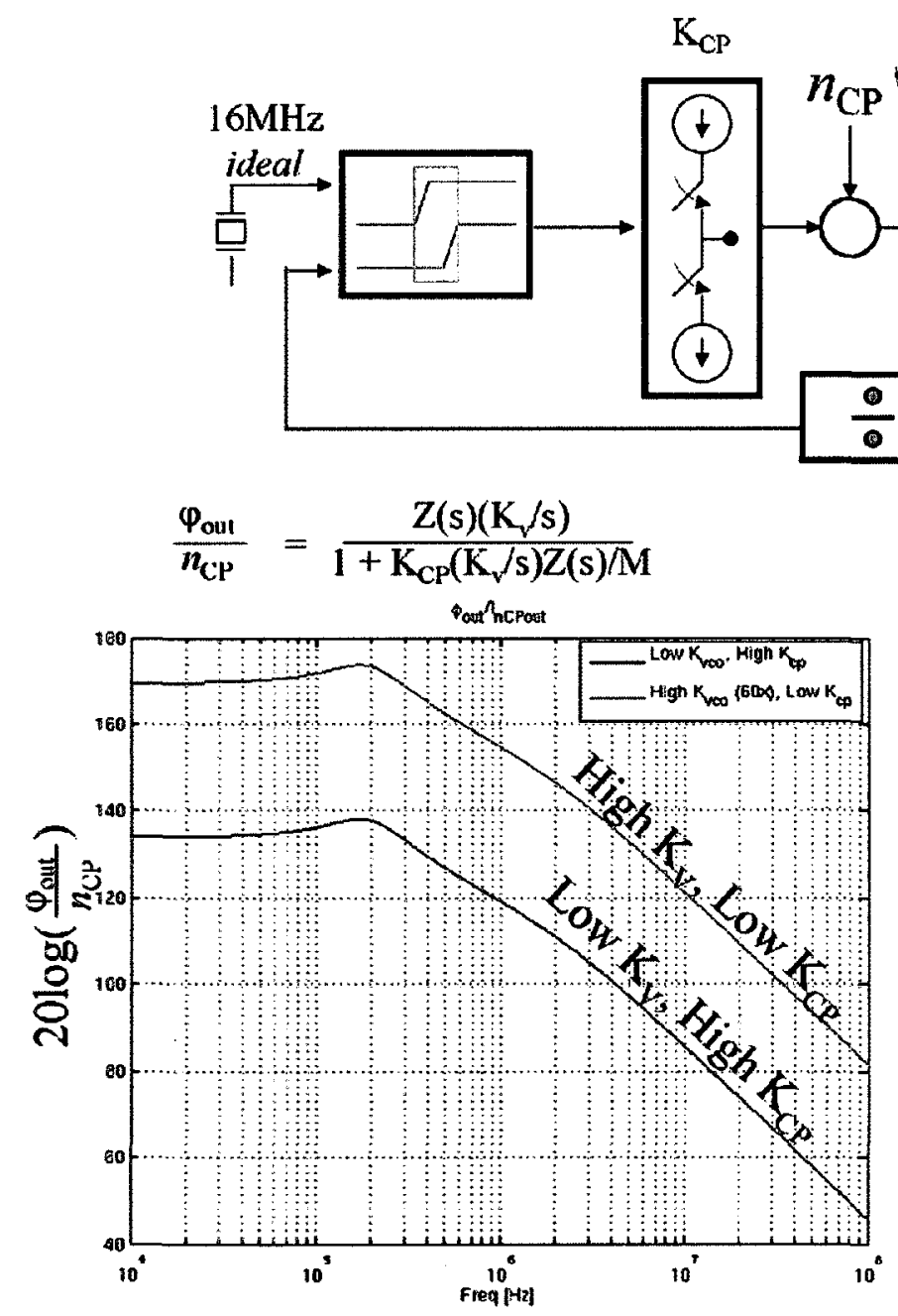

a) Charge-Pump Noise Transfer function

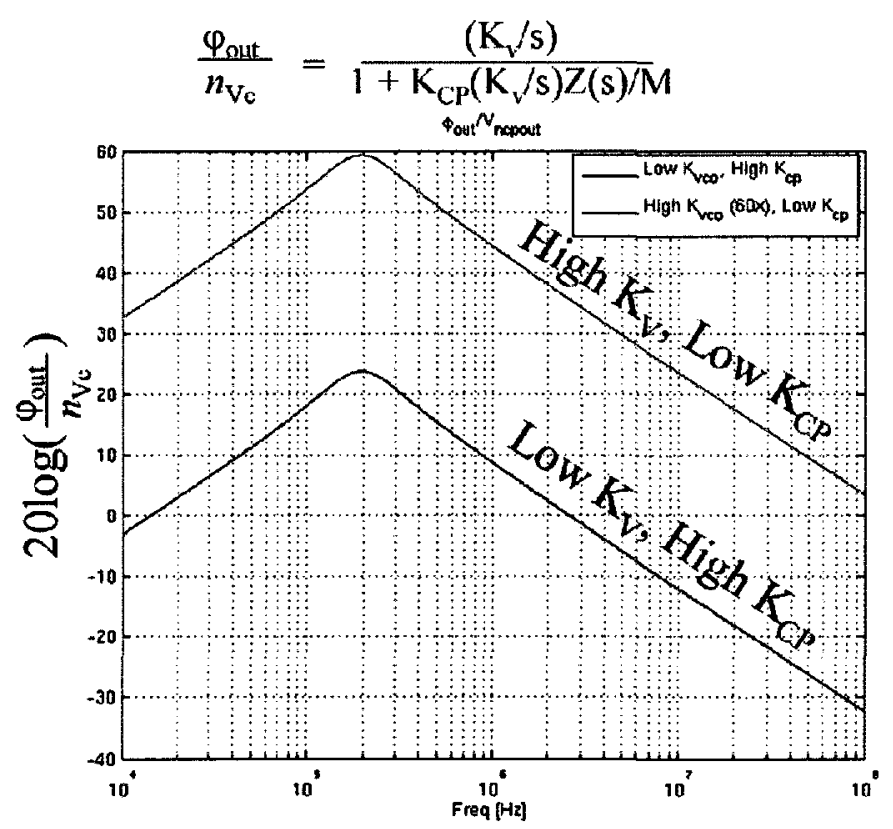

b) Tuning port Noise Transfer function

Figure 3.10: How VCO gain scales midstream noise: (a) transfer function to noise which is subjected to the filter, (b) transfer function to noise which is immune to the filter. Lowering $K_{V}$ and increasing $K_{C P}$ improve noise suppression from the charge-pump, filter, and front-end of the VCO.

The last section showed the equivalence of the presented architecture with an analog PLL with low VCO-gain $\left(K_{V} / N\right)$. As described in Chapter 2, low gain 
VCOs provide advantages in terms of noise immunity. The presented architecture effectively reduces $K_{V}$ to arbitrarily low levels by increasing the number of stages $\mathrm{N}$, and therefore realizes this advantage without sacrificing VCO range.

The analog control to the VCO is susceptible to a variety of noise sources. Since this control voltage is high-impedance and normally has a very limited swing, even moderate coupling can cause proportionally drastic changes in the control level, which is then magnified by the VCO gain. Intuitively then, low $K_{V}$ would seem to make the system less sensitive to these disturbances. In addition to this natural explanation, the mathematical transfer function and simulation results will show that this is indeed the case, and that PLLs with low VCO gain can be made more resilient to various forms of noise.

When considering noise on the control node $V_{c}$, it is valuable to make a distinction between noise which is introduced before or after the loop-filter. The transfer function of noise on both these nodes is shown in Figure 3.10a and 3.10b respectively. Case (a) applies primarily to noise at the output of the charge-pump which is exposed to the loop-filter, whereas case (b) applies to noise from certain nodes in the loop-filter (which don't see a high-freq shunt to ground), and to noise in any active stages in the path to (or in) the VCO. In either case, significant benefits are achieved by decreasing $K_{V}$ with a corresponding increase in $K_{C P}$. The simultaneous reduction of $K_{V}$ and increase in $K_{C P}$ will keep the loop-bandwidth constant and reduce both high-frequency noise (from VCO and mid-stream effects) and low frequency noise (from the charge-pump). ${ }^{7}$

\subsection{System Level PLL Simulator}

In a separate effort (compared to Figure 3.7), a more elaborate system-level simulator was written to characterize more aspects of PLL behaviour and to include live processing of results in Matlab. The mixed-signal simulator was written in vanilla Verilog, with processing in Matlab to calculate theoretical transfer functions, visualize the jitter of the system, and plot jitter and phase-noise versus time and frequency. A block-diagram of the simulator is shown in Figure 3.11.

\footnotetext{
${ }^{7}$ The cost of increased $K_{C P}$ is generally a second order increase in the amount of noise introduced onto $V_{c}$, but it is more than compensated by the systems reduced response to this noise.
} 


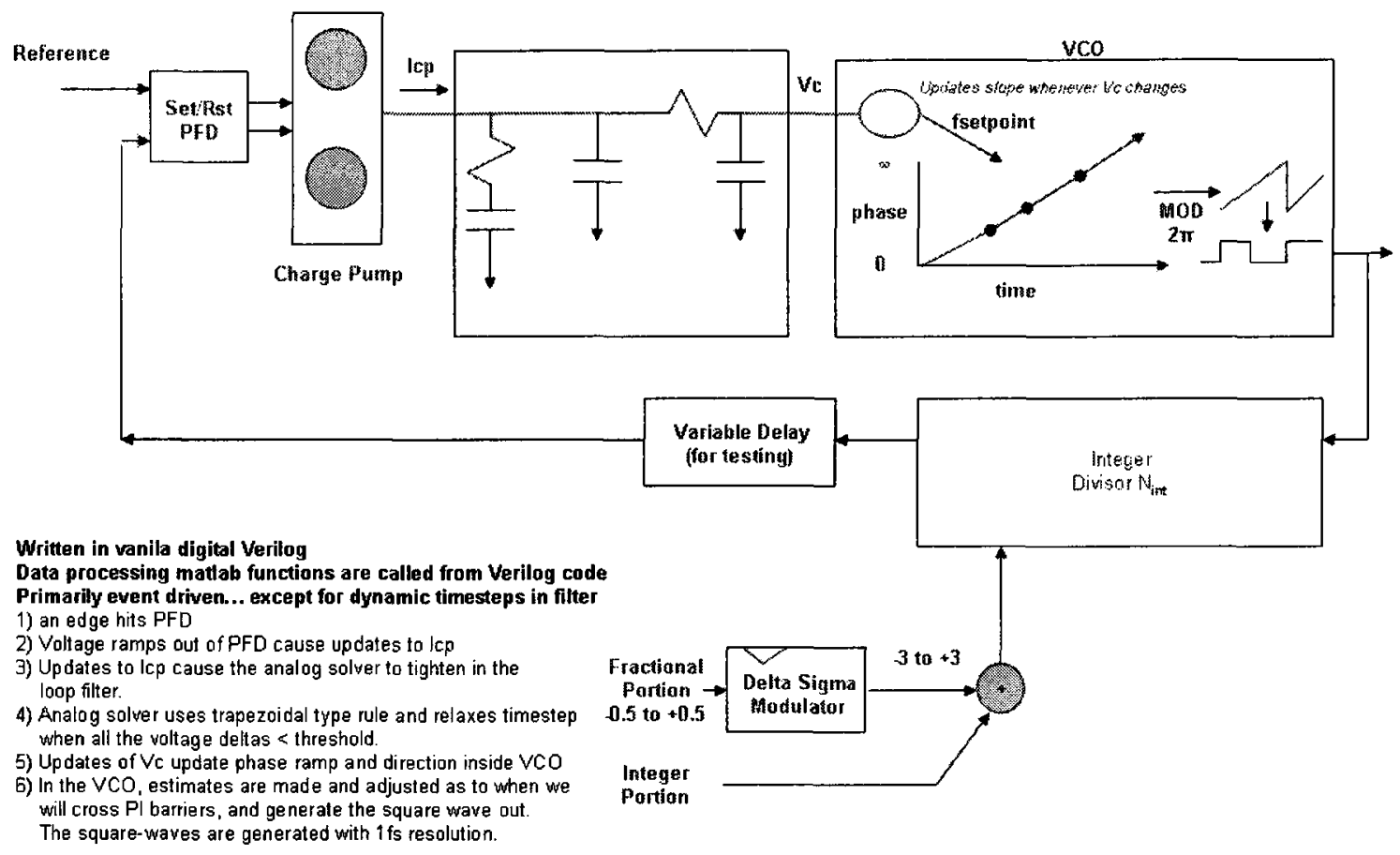

Figure 3.11: System Simulator: An elaborate dynamic time-step PLL simulator was developed, primarily to model lock-times and non-linear modulation effects in a very fast and controllable manner.

Verilog is a programming language, just like any other. It has access to real numbers, and though cumbersome, routines were developed to perform simple trigonometric functions for use in the simulator. As such, any model that might be written in C, matlab or simulink could also be written in verilog. One of the advantages of the verilog model, is that it allows the user to swap in actual hardware for much of the circuit as it becomes available.

Though modeling the PFD and divider are relatively straightforward, it took significant effort to accurately and efficiently model the VCO and the higher order continuous time analog filters. At each time-step, which is dynamically scaled, the analog solver in the loop-filter uses the voltages from the previous step to estimate the currents through each component of the loop-filter. Based on these current estimates it updates the node voltages and re-calculates the currents. It then takes the average of the two current estimates and updates the node voltages accordingly. One of the advantages of writing a special purpose simulator, is that the model is aware 
in advance when drastic events will take place, such as turning a current source from 0 to $I_{C P}$ in a few ps timespan. The simulator uses this information to warn the differential equation solvers to update their results, tighten their timesteps and prepare for the coming discontinuity. As activity settles out, the $\Delta$ voltages and currents in the filter decrease, and the simulation logic within the loop filter relaxes the time-step until another event occurs. With each update of $V_{c}$, the VCO must recalculate the oscillation frequency. The VCO model maintains a phase ramp which changes rate slightly depending on the control voltage. As the phase ramp approaches $\pi$ boundaries, the model prepares to transition the VCO output waveform from 0 to 1 or 1 to 0 . Despite the use of double-precision floating point numbers, it was necessary to use a number of techniques inside the VCO to prevent round-off errors from accumulating and distorting the simulation results. Code profiling shows that the loop-filter calculations consume approximately $70 \%$ of the simulation time, and the VCO consumes about $25 \%$. The accuracy parameters of the simulation can be scaled on the fly, with a corresponding change in run-time.

The running bench polls a set of approximately 40 different parameters from a text file. Updating any of these parameters is reflected within 10 reference cycles in the output. The text-file used to index the parameters is shown in Figure 3.12.

A number of different nodes are monitored and post-processed in matlab. A screenshot of the post-processing environment is shown in Figure 3.13.

The most important result from the simulator is simply a list of timestamps (with fs precision), which record the rising-edge strikes of the VCO. Referring to figure 3.14 , these timestamps are compared with an ideal free-running VCO at the target frequency. The error vs time is the integrated jitter measurement ${ }^{8}$. From this data, both a jitter histogram and FFT are generated, showing the traditional jitter and phase-noise plots familiar from lab instruments. A screenshot of this main summary window is shown in Figure 3.14.

A comparison of the simulation time necessary to run to $30 \mathrm{us}$ is shown in Figure 3.15 for a variety of abstraction levels. The developed PLL software simulates a locking PLL approximately 20,000x faster than an all transistor level model, and 300x faster than an ideal verilogA PLL. The simulation accuracy is also configurable onthe-fly and typically has a noise floor better than $-200 \mathrm{dBc} / \mathrm{Hz}$ with a $50 \mathrm{MHz}$ reference.

\footnotetext{
${ }^{8}$ This is also sometimes known as the long-term jitter measurement. See appendix D for more.
} 


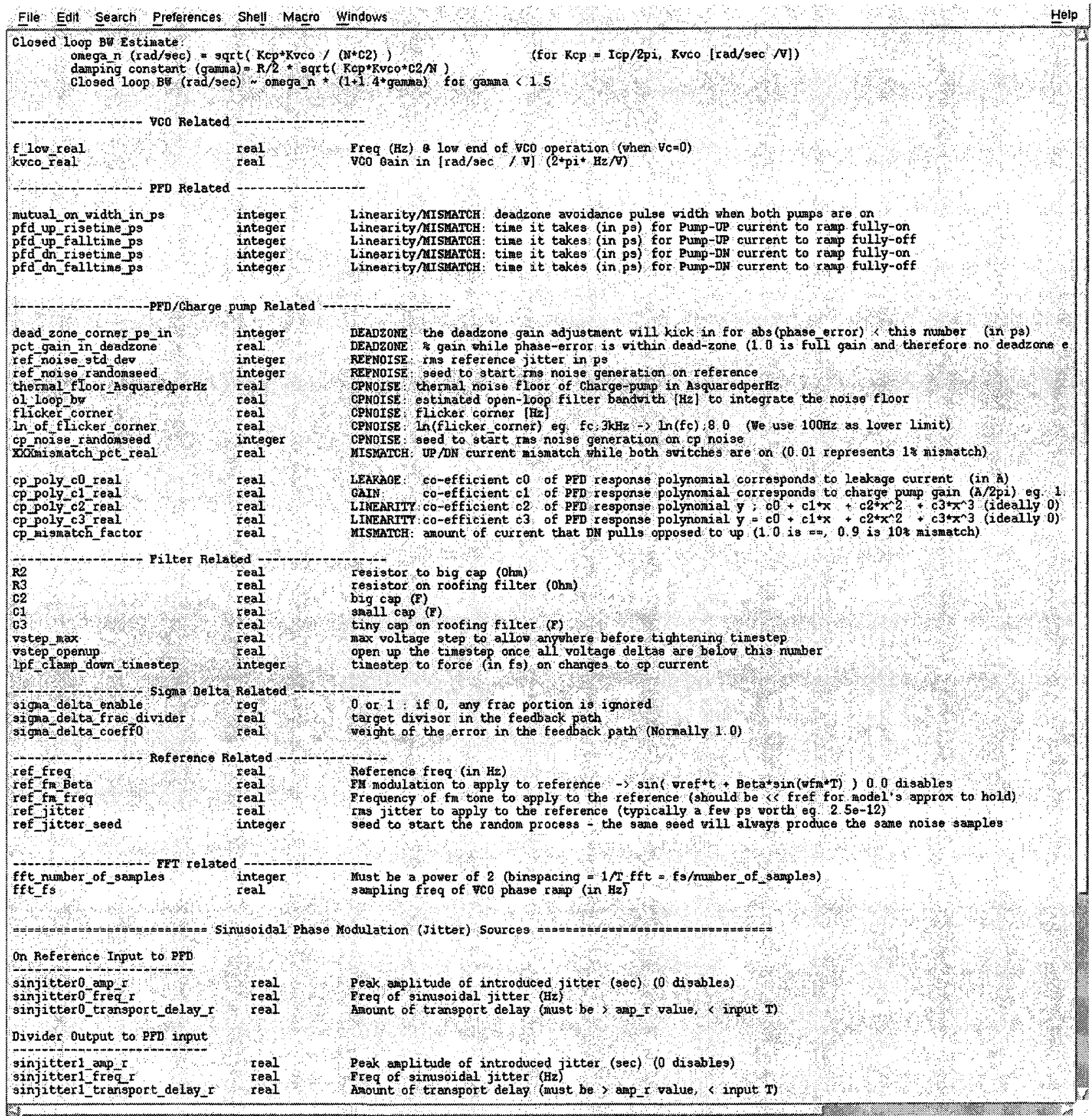

Figure 3.12: System Simulator Parameters: Parameters are constantly refreshed from a file, including noise levels of components, linearity specifications, dead-zone parameters, gain settings, loop-parameters, accuracy thresholds, etc. 


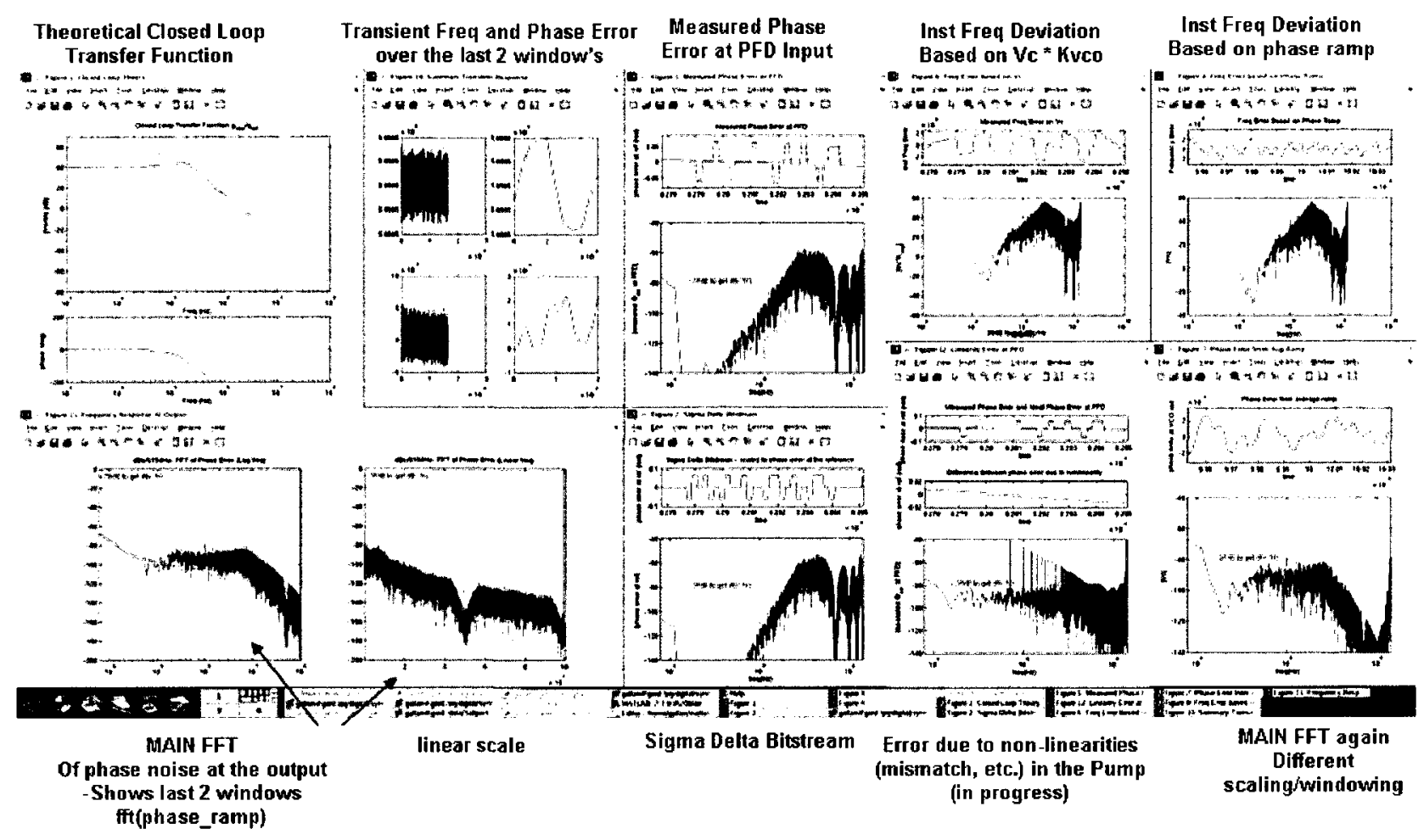

Figure 3.13: System Simulator Post-Processing: The Matlab processing environment analyzes the waveforms at various nodes of the PLL in both the time and frequency domain.

Only slight code modifications are required to account for any additional non-ideal effects the user wants to model, allowing significant flexibility. The simulator is used in the remainder of the chapter to illustrate the benefits of reduced VCO gain, in that it allows for reduced noise sensitivity via increases in $K_{C P}$ and/or can be used to reduce filter size.

\subsection{Simulation of Noise sensitivity vs $K_{V}$}

System level simulations were performed for both a conventional PLL and a PLL with $K_{V} / 60$ and $60 * K_{C P}$. To stimulate the model with a realistic noise source, a ring-oscillator was designed and its phase-noise was simulated to be $-108 \mathrm{dBc} / \mathrm{Hz}$ (a) $125 \mathrm{MHz}, 1 \mathrm{MHz}$ offset. This noise is input referred to the VCO control port by applying a scaling of $\frac{s}{K_{V}}=\frac{1 M * 2 * \pi}{K_{V}}$. A Gaussian random noise generator was then 


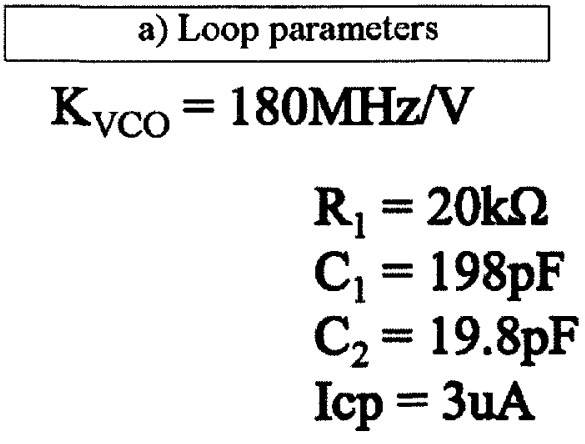

c) $3 \mathrm{D}$ eye diagram
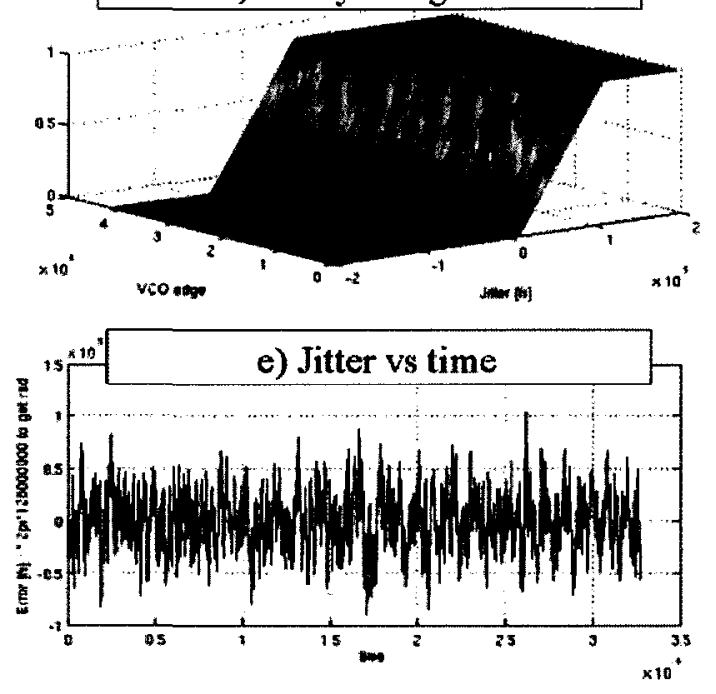

g) Phase Noise

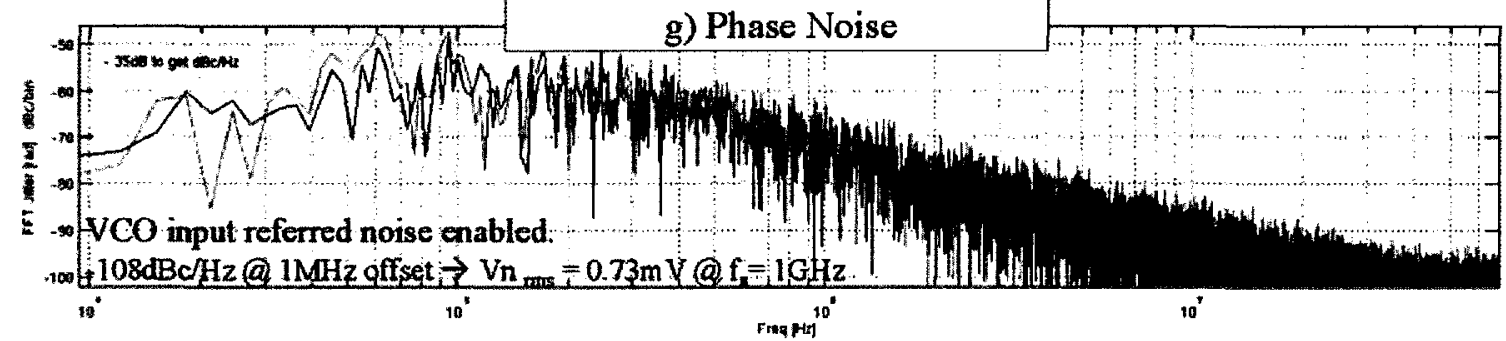

Figure 3.14: The main result from the simulator is based on the VCO rising-edge timestamps. From these, the jitter vs time (plot e), jitter histogram (plot f) and phase-noise (plot g) are all readily available.

scaled and introduced on the VCO tuning port to generate a flat spectral density of the appropriate power. This introduces a noise source of the appropriate power at the node in front of the VCO, at $n_{V c}$, indicated in Figure 3.10b. Found at the end of the chapter, Figures 3.16 (high $K_{V}$, low $K_{C P}$ ) and 3.17 (low $K_{V}$, high $K_{C P}$ ) 


\begin{tabular}{|l|l|c|}
\hline Simulator & Simulation Type & Sim Time to $30 \mathrm{~s}$ \\
\hline verilog & All verilog system simulator & $9 \mathrm{~s}$ \\
SpectreVerilog & All ideal verilog. Verilog-A & $46 \mathrm{~m}$ \\
SpectreVerilog & Real transmission gate resistors, ideal otherwise & $1 \mathrm{hr} 54 \mathrm{~m}$ \\
SpectreVerilog & Real supply models, transmission gate resistors, ideal otherwise & $2 \mathrm{hr} 17 \mathrm{~m}$ \\
SpectreVerilog & All real except CP & $21 \mathrm{hr}$ \\
SpectreVerilog & All ideal except CP & $12 \mathrm{hr}$ \\
\hline
\end{tabular}

Figure 3.15: Simulation Speedup of System Level Simulator: Time to simulate lock of a conventional PLL with different simulators and levels of abstraction. It takes only 9 seconds to simulate lock with the verilog system level simulator, whereas it takes 46 minutes with a verilog-A simulation that has equivalent model detail.

compare the resultant position of the VCO edges with respect to their ideal locations. The result over time is the jitter waveform, and the FFT of this shows the simulated phase-noise.

Figure 3.16 (high $K_{V}$, low $K_{C P}$ ) shows $\approx-90 \mathrm{dBc} / \mathrm{Hz} @ 100 \mathrm{kHz}$ offset, whereas Figure 3.17 (low $K_{V}$, high $K_{C P}$ ) shows $\approx-125 \mathrm{dBc} / \mathrm{Hz} @ 100 \mathrm{kHz}$, for a $35 \mathrm{~dB}$ improvement with a $60 \mathrm{x}$ reduction in $K_{V}$ and increase in $K_{C P}$. Comparing the probability density function of the jitter, the RMS variation appears to improve from about 25ps to $0.5 \mathrm{ps}$. The theory predicts an improvement of $20 \log _{10}(60)=35.5 \mathrm{~dB}$. Also note that the simulated phase-noise has the same form as predicted by Figure 3.10b. Since the noise was inserted to model that of a real VCO, it makes sense that it follows the expected VCO noise curve - with noise suppression in the loop (below $\omega_{0 d B}$ ) and a $20 \mathrm{~dB} / \mathrm{dec}$ rolloff (the $1 / \mathrm{s}$ term of the VCO) outside of the loop.

\subsubsection{Capacitance Reduction Benefits}

In section 2.4.2 it was shown that the level of the open-loop transfer function, and hence the loop bandwidth, is primarily determined by the ratio of $K_{C P} * K_{V} / C_{1}$. It was previously mentioned that reducing $K_{V}$ by a factor of $N$ lowers the loop bandwidth. At the time it was suggested that $K_{C P}$ could be increased by a factor of $N$ to compensate for the reduced loop bandwidth and provide low-frequency noise immunity. Instead of increasing $K_{C P}$ to restore the loop bandwidth, it is possible to reduce the integration capacitance $C_{1}$ by $\mathrm{Nx}$ instead, and thus save area. ${ }^{9}$ In Figure $3.18, K_{V}$ is reduced by $60 \mathrm{x}$ without a corresponding increase in $K_{C P}$. To keep

\footnotetext{
${ }^{9}$ It is also necessary to scale up $R_{1}$ to keep the stabilizing zero in the same location.
} 
the loop BW constant, the filter components are resized such that all caps are $60 \mathrm{x}$ smaller, and the resistance is $60 \mathrm{x}$ larger. Note that the loop dynamics in Figure 3.18 match those of Figures 3.16 and 3.17 , despite a loop filter which is $\approx 60 \mathrm{x}$ smaller.

In general then, there is some compromise to be made. The presented architecture allows the effective reduction of $K_{V}$ by $N \mathrm{X}$ while still maintaining application requirements. This reduced VCO gain leads to reduced sensitivity to noise on the analog control node $V_{c}$. To maintain the loop bandwidth with the effectively reduced $K_{V}, K_{C P}$ can be increased (reducing low-frequency noise) and/or $C_{1}$ can be decreased (reducing area). Normally, some compromise between these two benefits would be made.

\subsection{Using the Architecture in DLLs instead of PLLs}

Though most of the discussion thus far has focused on the use of the system in PLL loops, it was originally conceived for low-complexity DLLs where its implementation is far simpler. In DLLs, there is normally only one integrator term in their transfer function and they are unconditionally stable. This means that their loop-filter is merely a capacitor, and there are no stability requirements to be met. In fact, for DLLs it is possible to forgo the use of a switched capacitance altogether, and merely increase the number of stages $\mathrm{N}$ to the point where the total parasitic capacitance $\left(N * C_{2}\right)$ is equal to the intended integration capacitance of a single voltage system. In practice, this would be less efficient, however, than using the filter switching system introduced in the next chapter.

\subsection{Summary}

A new cascaded charge-pump structure, formed with simple tri-state gates, has been introduced which allows arbitrary reductions in VCO gain without sacrificing frequency range. Aside from this main benefit, the architecture can be implemented with entirely digital cells, is amenable to automatic place\&route and has significant scaling advantages over other architectures.

Simulators have been written to validate this circuit configuration, and others, in a fraction of the time it would otherwise take. These simulators also provide 
the ability to model non-linear effects which are difficult to capture analytically Furthermore, the simulators provide an analysis of the PLL node-by-node, allowing the designer to visualize the various interactions in both the time and frequency domain. The simulators have been used to show that an N-stage cascaded charge-pump has the same loop dynamics as a conventional analog PLL with VCO gain $K_{V} / N$.

It has been shown via theory in Chapter 2, and via simulation here, that the reduction in $K_{V}$ afforded by the CCP can be traded for increased charge-pump gain and thus better noise performance, or for reduced filter capacitance and smaller filter sizes. Further, any improvements in phase-noise due to increased CP gain extend the optimal loop-BW, allowing further size reduction. 


$$
\begin{aligned}
\mathrm{K}_{\mathrm{VCO}}=180 \mathrm{MHz} / \mathrm{V} \\
\mathrm{R}_{1}=20 \mathrm{k} \Omega \\
\mathrm{C}_{1}=198 \mathrm{pF} \\
\mathrm{C}_{2}=19.8 \mathrm{pF} \\
\mathrm{Icp}=3 \mathrm{uA}
\end{aligned}
$$
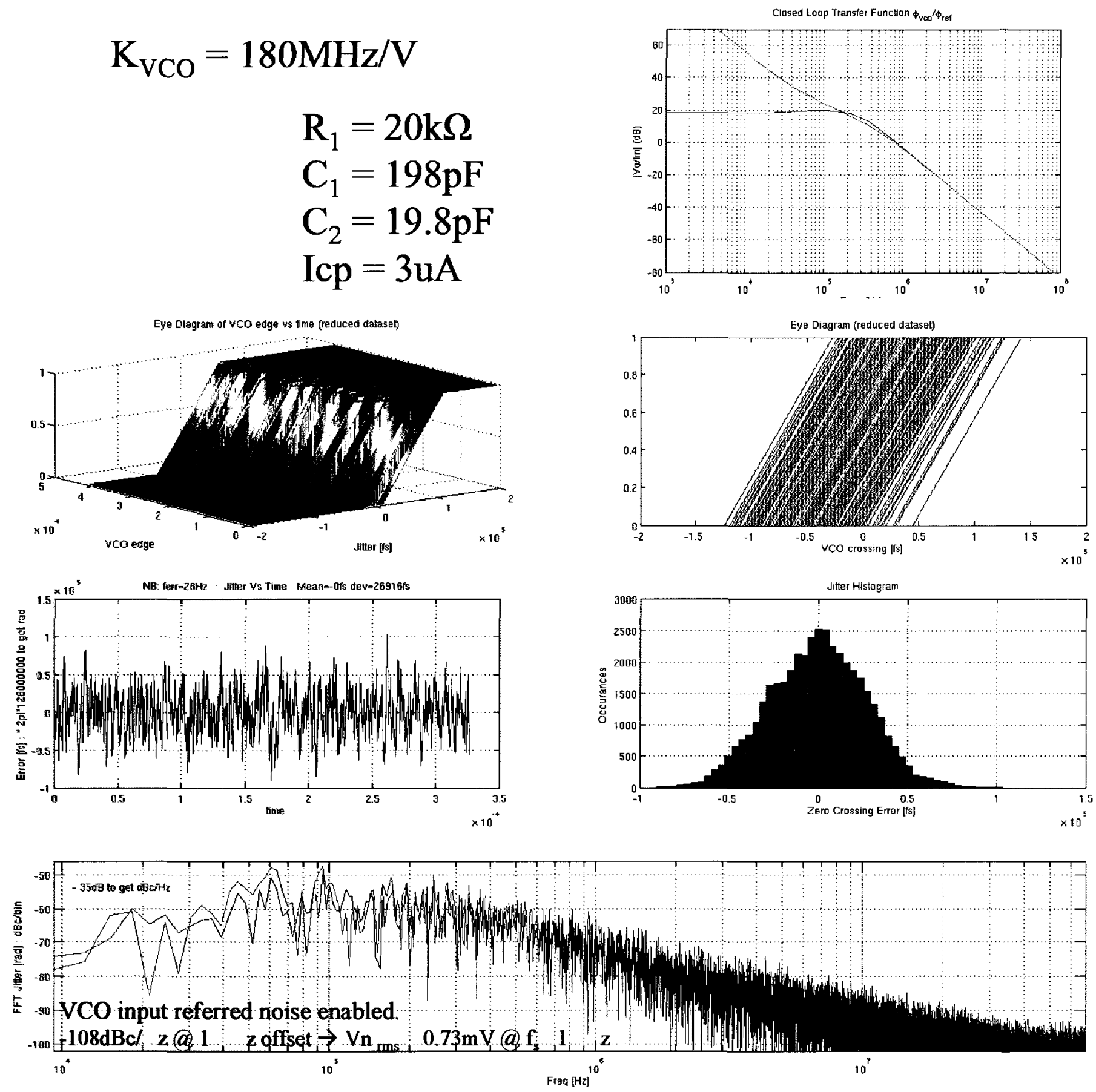

Figure 3.16: Simulation Results: A typical analog PLL (High $K_{V}$ and large caps), stimulated with simulated VCO noise, resulting in phase-noise of $\approx-90 \mathrm{dBc} / \mathrm{Hz} @$ $100 \mathrm{kHz}$ offset. 


\section{$\mathrm{K}_{\mathrm{VCO}}=3 \mathrm{MHz} / \mathrm{V}$}

$$
\begin{aligned}
& \mathrm{R}_{1}=20 \mathrm{k} \Omega \\
& \mathrm{C}_{1}=198 \mathrm{pF} \\
& \mathrm{C}_{2}=19.8 \mathrm{pF} \\
& \mathrm{Icp}=180 \mathrm{uA}
\end{aligned}
$$
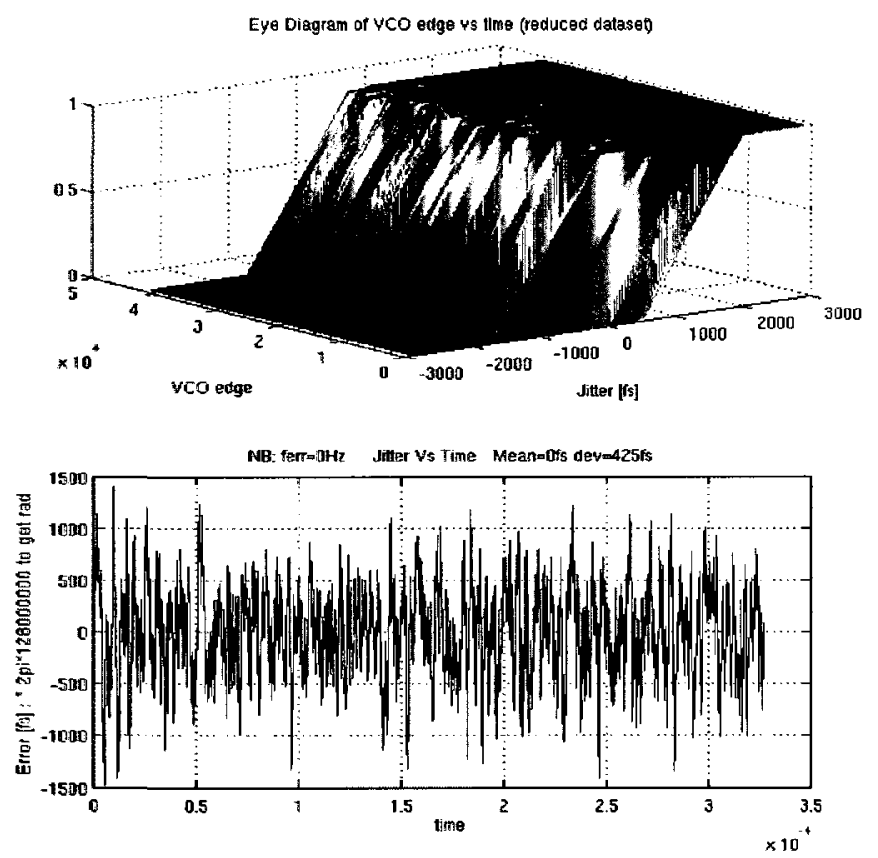
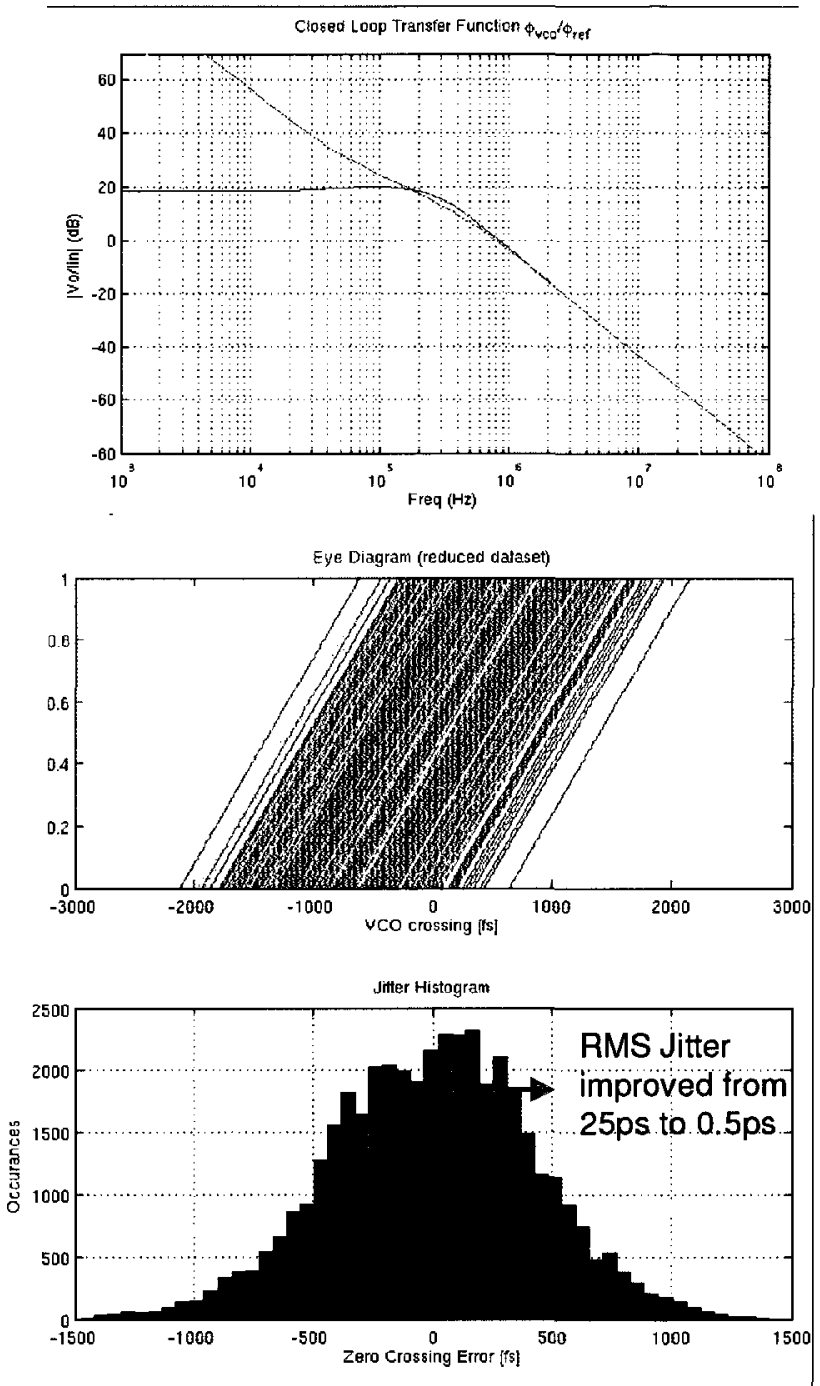

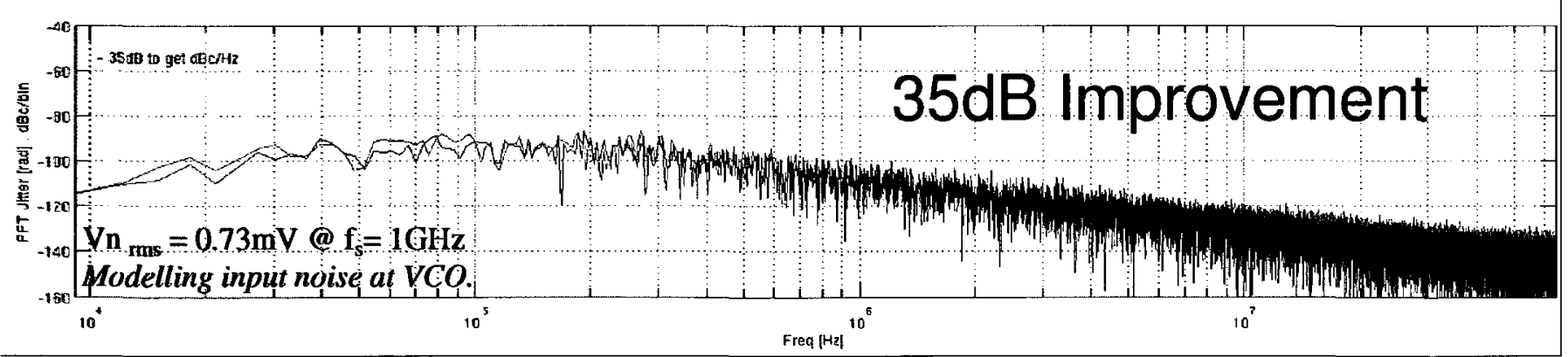

Figure 3.17: Simulation Results: An analog PLL with low $K_{V}$ and high $K_{C P}$, stimulated with simulated VCO noise, resulting in phase-noise of $\approx-125 \mathrm{dBc} / \mathrm{Hz} @ 100 \mathrm{kHz}$, for a $35 \mathrm{~dB}$ improvement. 


\section{$\mathrm{K}_{\mathrm{VCO}}=3 \mathrm{MHz} / \mathrm{V}$}

$$
\begin{aligned}
& \mathrm{R}_{1}=1200 \mathrm{k} \Omega \\
& \mathrm{C}_{1}=3.3 \mathrm{pF} \\
& \mathrm{C}_{2}=330 \mathrm{fF} \\
& \mathrm{Icp}=3 \mathrm{uA}
\end{aligned}
$$
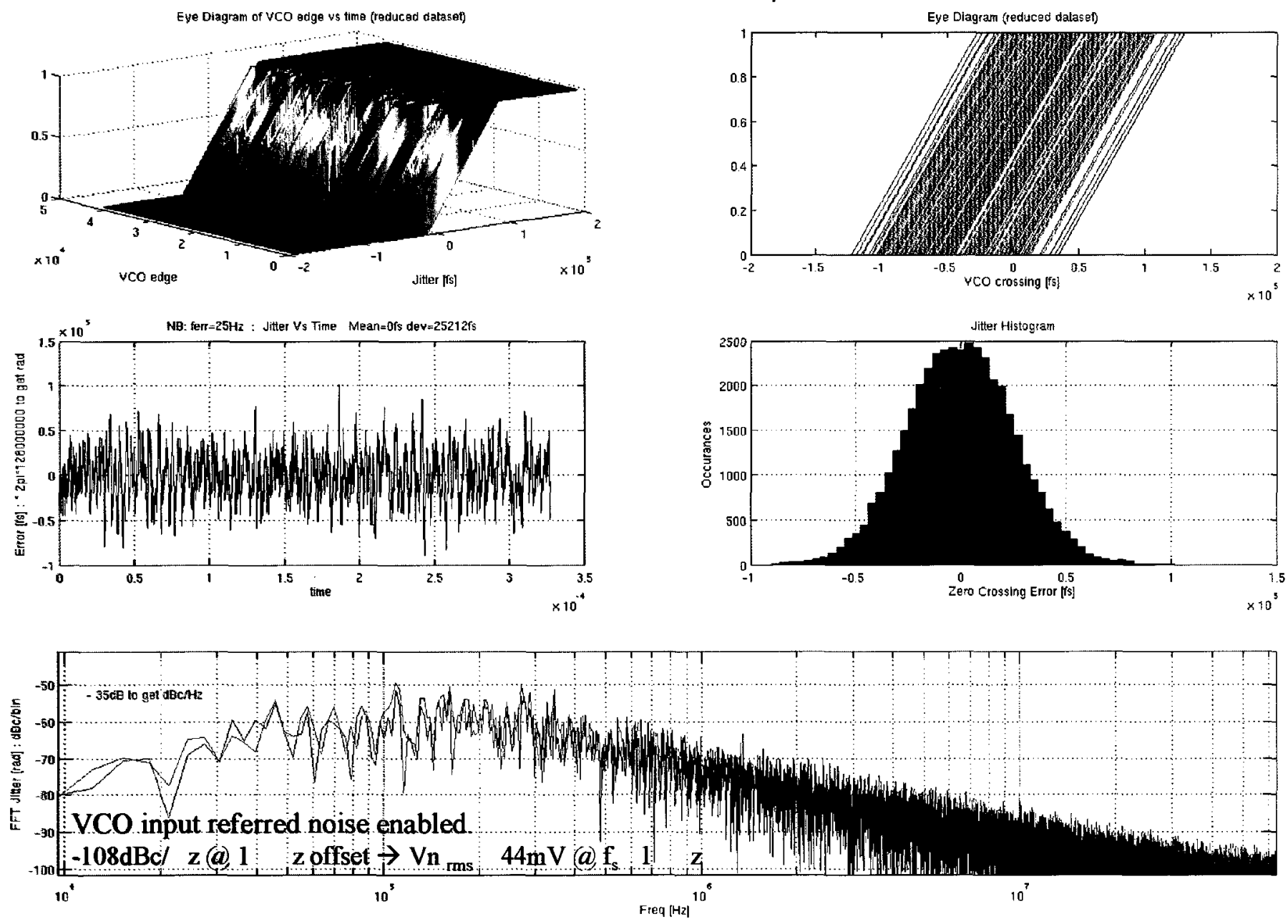

Figure 3.18: Simulation of Low Gain VCO with Small Caps (instead of large $K_{C P}$ : While maintaining the same loop-BW, filter capacitance can be reduced, saving area. (Forgoing noise improvements that would have come from an increased $K_{C P}$.) 


\section{Chapter 4}

\section{Circuit Implementation}

\subsection{Overview}

This chapter covers a number of details regarding the cascaded-pump structure.

After a brief review of the conceptual version, the chapter will introduce an inverting thermometer coded configuration. This inverting configuration is more difficult to visualize, but it simplifies the hardware and allows the circuit to avoid short-circuit currents which would otherwise plague the architecture. Further simplifications will also be shown, which reduce the core charge-pump circuitry to only 4 minimally sized transistors/stage. A few examples will also be presented about how a VCO or delay-line can be modulated by a mixed-signal vector similar to that produced by the CCP.

In Chapter 3, it was suggested that the current sources in the cascaded pump use simple tri-state drivers. By avoiding controlled current sources, the circuit can be made simpler and smaller. Without the well controlled current though, it is important to examine the implications of a poor source resistance $R_{C P}$. That is done here, and we also outline a method to determine the gain of the charge-pump, and to determine how consistent that gain is as the analog control is passed from stage to stage.

Thus far, little attention has been paid to the filter element(s) which must be connected to the node of the charge-pump under analog control. Since the analog node will always be moving during acquisition or temperature drifts, it is necessary to have either all nodes filtered (which would be wasteful), or to dynamically rotate the filter section to the area of interest. This takes a great deal of care, since the filter rotation should be done gracefully without disturbing the loop. It is a further complication that static CMOS digital logic cannot be fed with potentially analog 
signals - or short-circuit currents would develop. Instead pass-transistor logic is used, in combination with specially chosen sequencing of when and where a filter can be disconnected in one location and reconnected elsewhere.

To guard against charge-leakage, a circuit will be introduced to tie-off the nodes away from the analog transition region of the code to stable voltage references - potentially to VDD and GND. Having done this, it is important to evaluate the supply noise sensitivity of the circuit.

To reduce charge feedthrough and manipulate the gain and mismatch characteristics of the CCP, a number of preconditioning circuits will be discussed that can optionally go between the PFD and the CCP.

Since the frequency of the loop is roughly determined by the digital state of the thermometer-code, it can be useful to save and recall it for quick reacquisition. One method would be to add a latch to each node, but this would double the active hardware requirements per stage. It will be shown that given the circuits discussed earlier in the chapter for sharing filter sections and tying off nodes to stable references, only three latches will be necessary to save the state of the entire line, regardless of the number of stages.

\subsection{Simplifying the Cascaded Charge-Pump Hard- ware}

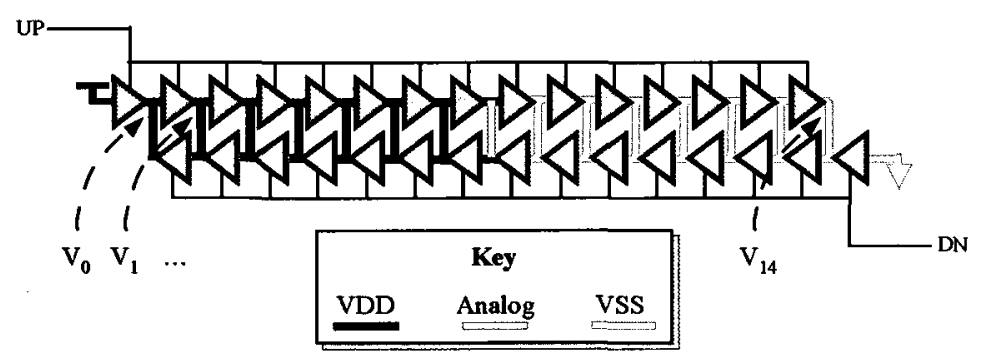

Figure 4.1: Tri-State buffer implementation of cascaded charge-pump

Reviewing what was given in Chapter 3, in its simplest conceptual form, the cascaded charge-pump is made by coupling two tri-state delay-lines together in opposite directions, as shown in Figure 4.1. Note that the primary inputs to each side 
of the tri-state chains are constants ( 0 and 1$)$, but the drive-enable signals are connected to the UP and DN control signals from the PFD. When the DN signal is asserted, the lower delay chain is enabled, and zeros will be driven from right to left. Similarly, when UP is asserted, the top delay chain attempts to drive ones from left to right. In practice, a competition ensues between the top and bottom delay-lines, which drive from opposite directions. Given an initial example codeword, such as $11111 \uparrow 00000000$, and examining Figure 4.1, one sees that if, on the next phasedetector output, UP and DN are asserted simultaneously, both the top and bottom delay-lines will agree about the value for all nodes except at the transition point $(\uparrow)$. Here, they compete. The top line works to charge the node, and the bottom line works to discharge it. For this net, the situation mirrors that of a regular charge-pump.

\subsubsection{Inverting Thermometer Codes}

Though conceptually very simple, the structure of Figure 4.1 is not recommended. Standard-cell tri-state buffers typically have a conventional inverter at the input stage. In the cascaded charge-pump, a few nets may maintain stable analog (mid-range) values and if these are passed into a CMOS inverter, large short-circuit currents will be generated, wasting power.

It is possible to replace the buffers in the chain with inverters. Though it seems odd to the eye, this inverting thermometer code is just as valid provided that every second node in the string controls an active-low element in the VCO or delay-line. In such an inverting code, shown in Figure 4.2, every second node is flipped in polarity. This removes the short-circuit problem (since every active stage is now tri-stateable), reduces the hardware, and also improves linearity since the overlap between control nodes is more extensive.

\section{$\begin{array}{lllllllll}1 & 1 & 1 & 1 & 1.8 & .2 & 0 & 0 & \text { Non-inverting code }\end{array}$ fip! fip! fip! fip! \\ $\begin{array}{lllllllll}1 & 0 & 1 & 0 & .8 & 8 & 0 & 1 & \text { Inverting code }\end{array}$}

Figure 4.2: Comparison of a regular and inverting thermometer code

A DLL which uses the cascaded charge-pump in an inverting configuration is pictured in Figure 4.3. It was in this inverting configuration, that the simulation was 

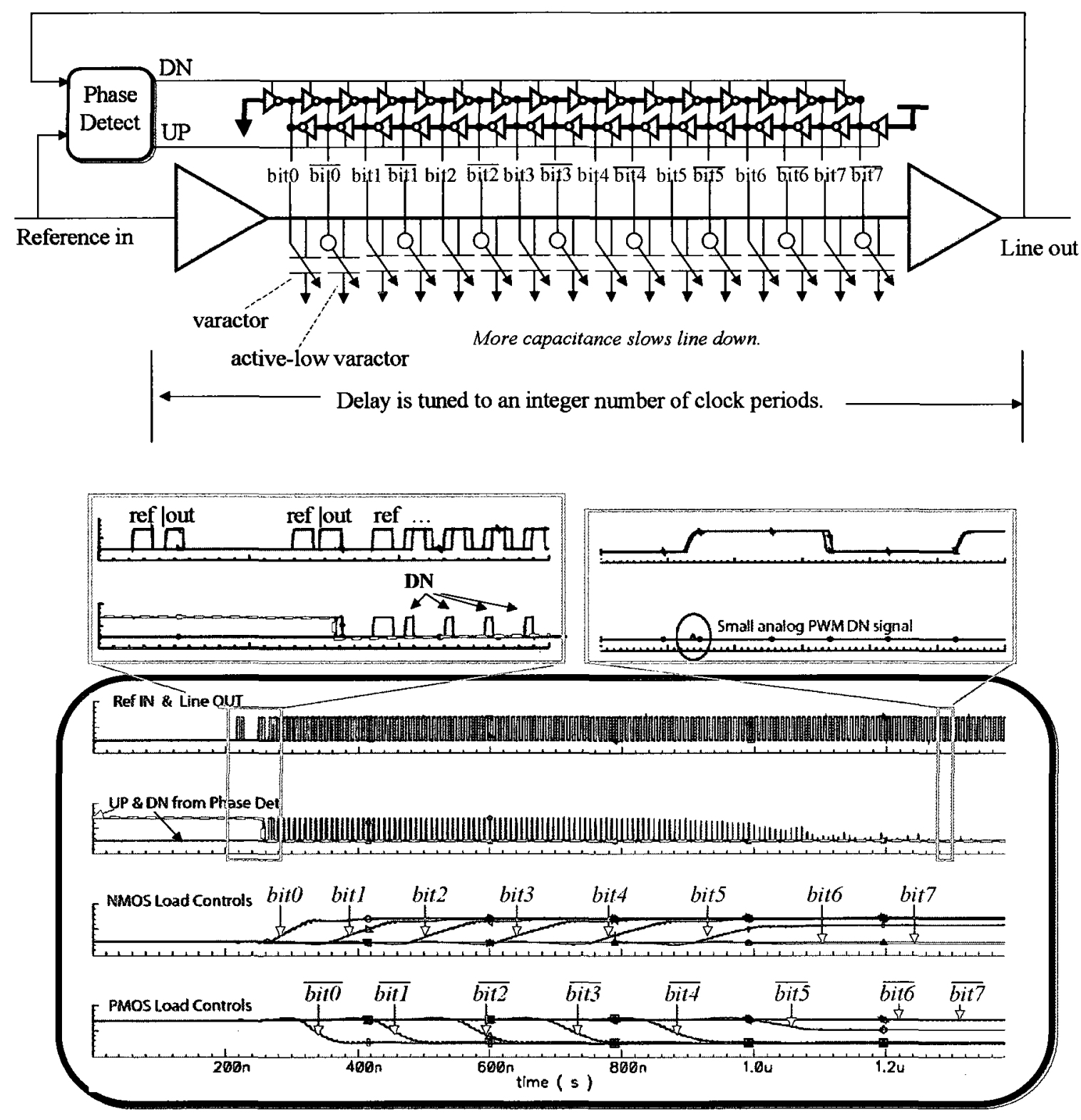

Figure 4.3: DLL Configuration of an Inverting cascaded charge-pump 
performed to generate the earlier results of Figure 3.5. In the earlier example, the details of the inverting configuration were not shown in order to simplify the circuit description for the reader. This figure shows the progression of both the active-high and active-low nodes of the thermometer code. As the phase-detector issues repeated DN pulses, the top delay-line operates in short-bursts. Over time, it charges bit $t_{0}$, discharges $\overline{b i t_{0}}$, charges $b i t_{1}$, etc... As the thermometer code progresses, more and more capacitance is exposed onto the delay-line, slowing it down. As the controlled delay approaches the reference period, the pulse widths from the phase-detector are reduced, and the progression of the thermometer code slows. Eventually, the loop settles into lock with 2 nodes (bit $t_{5}$ and $\overline{b i t_{5}}$ ) having clear analog values.

\subsubsection{Removing redundant transistors and halving the cir- cuit size}

If the cascaded charge-pump is implemented with standard tri-state buffers or inverters, over half of the transistors are redundant. Complexity and power consumption can therefore be reduced by introducing custom cells.

In order to illustrate, assume that the cascaded charge-pump is made with conventional (non-inverting) tri-state buffers as in Figure 4.1. Each buffer has 6 primary transistors, +2 on an internal inverter for the drive control signal. Because the upper delay-line is only responsible for charging nodes, and the lower delay-line for discharging them, it can be shown that only 3 transistors per buffer would be required, reducing the filter's active components to $\frac{3}{8}$ ths of their original size.

In the inverting cases, it is also true that half of the transistors are redundant. For the moment, consider just the top delay-line in Figure 4.4. It is responsible for slowing the delay elements, which is accomplished by charging the ODD lines, and discharging the EVEN lines. There is little reason why the top delay-line should need to charge EVEN lines, and discharge ODD lines, which is the responsibility of the competing delay-line when an UP signal is asserted. All of the transistors in the top delay-line which charge EVEN nets and discharge ODD nets can therefore be eliminated. The same argument can be used to eliminate all of the transistors in the bottom delay-line which would charge ODD nets, and discharge EVEN nets. The redundant transistors are shown, in gray, in Figure 4.4b. 


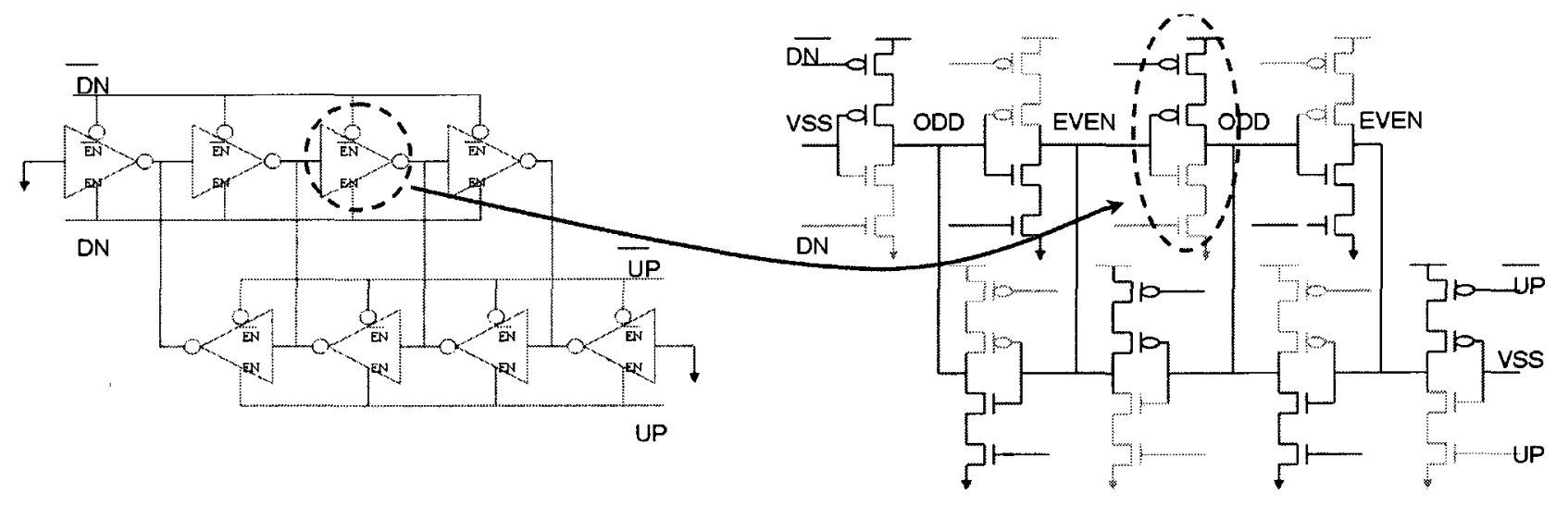

(a) Gate Level

(b) Transistor level, showing redundant transistors

Figure 4.4: Removing redundant transistors in the cascaded charge-pump

\subsection{VCO Modulation}

The control vector consists of a large number of nodes at their digital extremes, but with one or two of them hovering at stable analog values. Illustrated in Figure 4.5, a control vector of this sort can then be coupled to an oscillator or delay-element in a number of ways to modulate frequency or delay. In Chapter 5, a complete lowpower PLL will be presented where the VCO uses MOS varactors (voltage controlled capacitances), as shown in Figure 4.5b.

Though the sum of control voltages from the cascaded charge-pump is quite linear, this control vector must then be coupled to an oscillator or delay-line. Ultimately, the linearity of the system is determined by the response of the control string in combination with the VCO response. Depending on the degree of linearity required, or equivalently, how consistent the loop-dynamics must be across the operating range, the linearity of the VCO may or may not pose a design challenge. In practice, $K_{V}$ of typical VCOs vary by $\approx 2 \mathrm{x}$ across the control range. Due to the vectored and overlapping nature of the multi-node structure generated by the CCP, it may reasonably mitigate some of the otherwise troublesome non-linear effects of $K_{V}$ in single control voltage systems. 


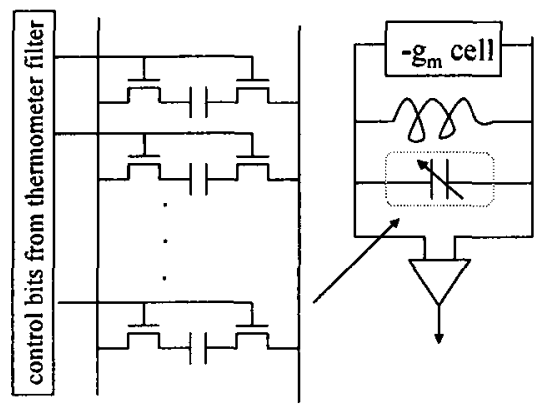

(a) LC oscillator control

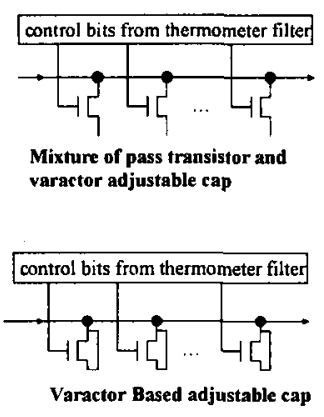

(b) CMOS delay control
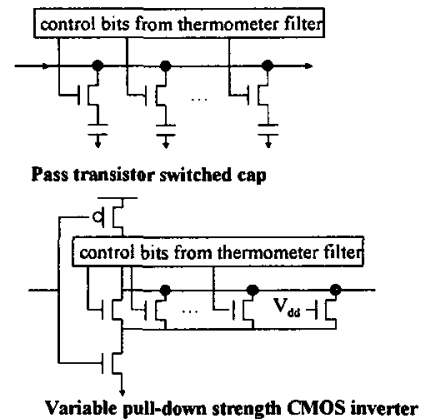

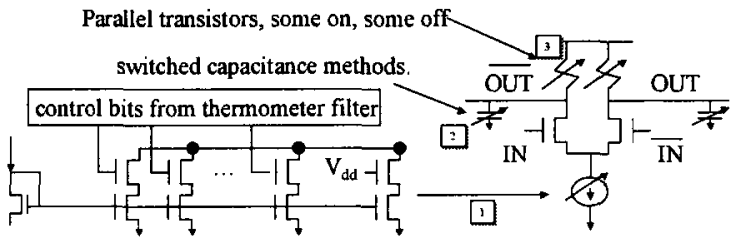

Adjust Current Source 11 or Adjustable Capacitive Load 2 or Adjustable Resistive Load

(c) CML delay control

Figure 4.5: Controlling VCOs and delay elements with a thermometer code

\subsection{Gain, Source Impedance and Consistency}

Like conventional error-integration techniques, the cascaded charge-pump can be broken into a charge-pump and loop-filter. In this section, the important charge-pump characteristics are discussed.

\subsubsection{Finite Current-Source Impedance}

An ideal charge-pump is a switched current-source. The parallel source resistance of the current-source should be infinity, and the switch should be ideal $\left(R_{o n}=0, R_{o f f}=\right.$ $\infty)$ with no turn-on or turn-off delay and mid-point switching threshold. Of course, practical charge-pumps exhibit none of these features. In the 'off' state, the switches have some finite resistance which contributes to leakage. This will be ignored for the time being. In the 'on' state there is inevitably some switch resistance and 
finite current-source resistance which, as illustrated in Figure 4.6, can be combined and modeled as an ideal switch in combination with an ideal current source and large parallel resistance $R_{C P} .{ }^{1}$ With ideal switches, the gain of the charge-pump is $K_{C P}=I_{C P} / 2 \pi$.

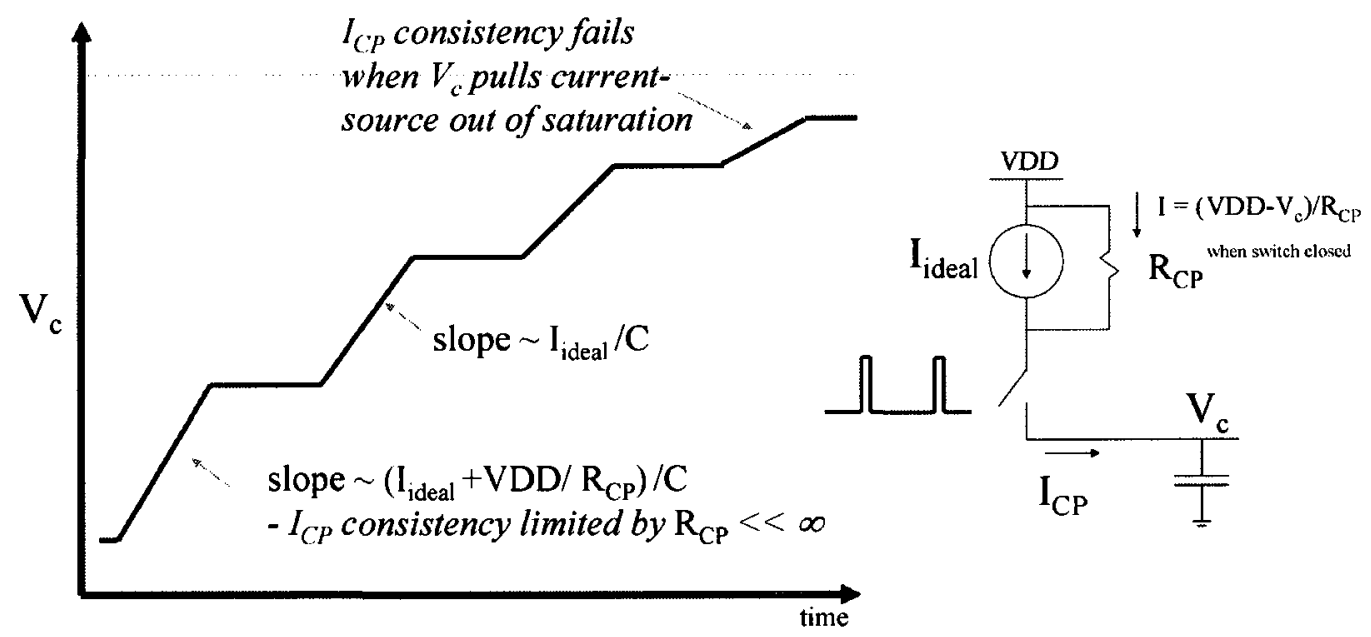

Figure 4.6: Modeling Non-Ideal Charge-Pumps: $R_{C P}$ and Non-Linearity: With a non-ideal current source or series resistance between the charge-pump and $V_{c}$ the amount of current sourced or sinked into the loop-filter for a particular pulse will not be constant. Instead it will depend on $V_{c}$. The result is that the charge-pump gain $K_{C P}$ will depend on the particular lock voltage $V_{c}$.

The finite source resistance $R_{C P}$ of a charge-pump has two main effects, both of which are illustrated in Figure 4.7.

\section{Pole Shifting of $\omega_{p 1}$}

With a shunt resistance $R_{C P}$ across the current source in Figure 4.6, a current divider is formed between the loop-filter and this source resistance. This current division can be modeled with the transfer function $\frac{V_{c}}{I_{\text {ideal }}}=\frac{R_{C P}}{1+s R_{C P} C}=\frac{R_{C P}}{1+s / w_{p 1}}$. With an ideal charge-pump, since $R_{C P}=\infty, \omega_{0}=1 / R_{C P} C=0$. In a PLL this pole combines with the VCO's pole at $\omega=0$ and results in an immediate phase-shift of $-180^{\circ}$ and a $-40 d B / d e c$ magnitude roll-off.

\footnotetext{
${ }^{1}$ Using the Thevinin equivalent circuit, this circuit could also be modeled as a voltage source in series with the same large resistance $R_{C P}$, and so can be considered a 'voltage-mode' charge-pump.
} 


\section{Type I Loop-Effects: Low $\mathrm{R}_{\mathrm{CP}}$}

$\phi_{\text {out }} / \phi_{\text {ref open-loop }}$

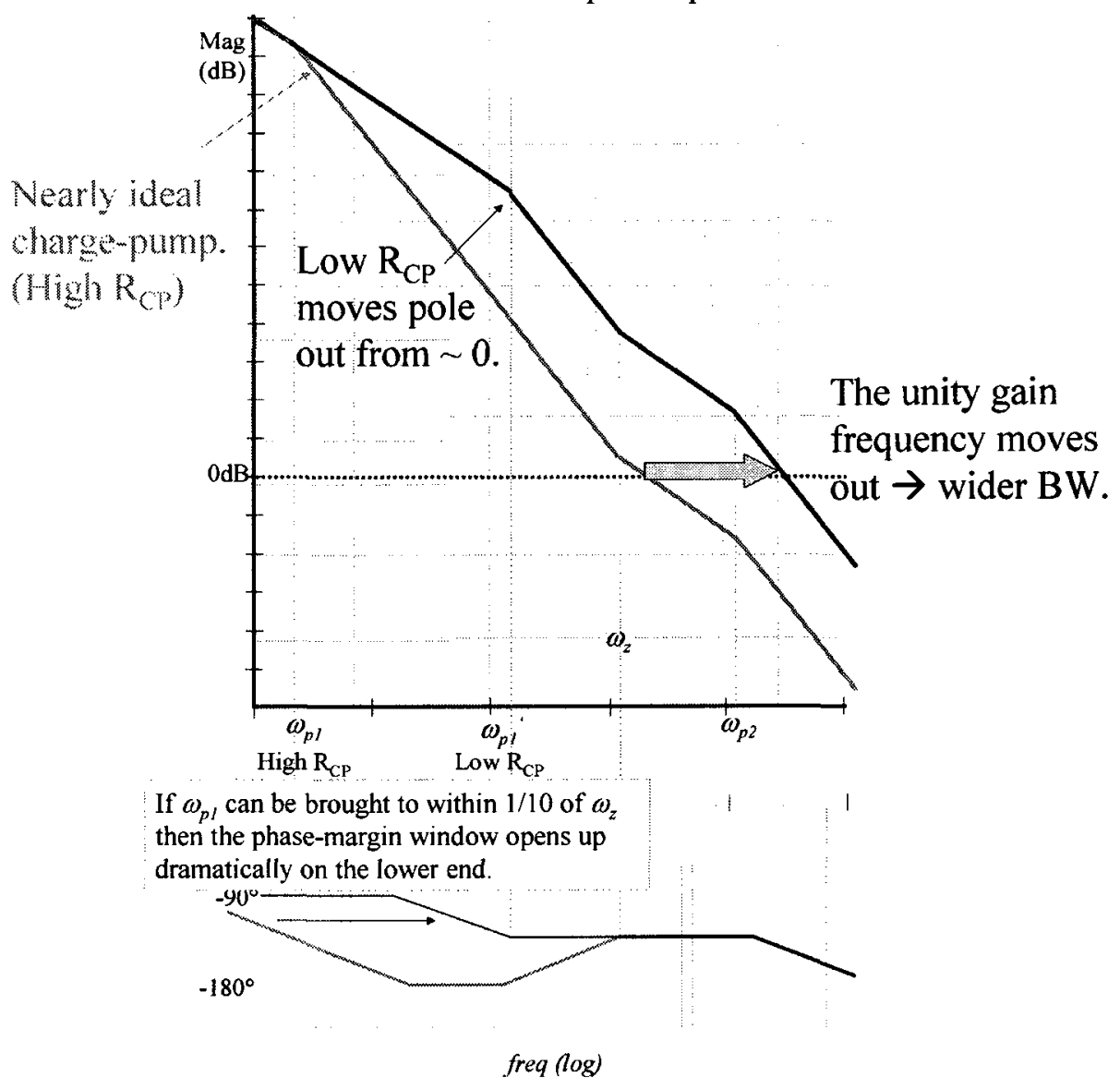

Figure 4.7: Effect of low charge-pump resistance $R_{C P}$ on loop-dynamics

Type II PLLs are characterized by these two poles at $\omega \approx 0$ and therefore, as covered in Section 2.4.1, require the addition of a zero to ensure stability. If $R_{C P}$ is finite, it combines with the filter capacitance and shifts the charge-pump's pole $\omega_{p 1}=0$ out to $\omega_{p 1}^{\prime}=1 / R_{C P} C$. This shifting 'partially' converts what was a Type II PLL to a Type I (with only one pole at $\omega=0$ ). All other things being equal, this will extend the loop-bandwidth. 
A potential advantage of the Type I architecture is an increased stability margin. If $\omega_{p 1}$ is brought out to within $\approx$ two decades of the $0 d B$ crossing point, $-180^{\circ}$ of phase-shift cannot occur before $\omega_{0 d B}$ and it will ensure loop-stability. ${ }^{2}$

Though stability margin can be increased, it comes at a cost. The lowfrequency magnitude roll-off is reduced from $-40 \mathrm{~dB} / \mathrm{dec}$ to $-20 \mathrm{~dB} / \mathrm{dec}$ until the pole $\omega_{p 1}$ is reached. Since the low-frequency VCO noise is scaled by the inverse of this curve (Figure 2.6), the VCO noise at frequencies below $\omega_{p 1}$ will be reduced by only $-20 d B / d e c$, rather than $-40 d B / d e c$.

\section{Non-constant $K_{C P}$}

In the ideal charge-pump, the switched current $I_{C P}$ should be constant regardless of $V_{c}$, thus leading to constant $K_{C P}$ and consistent loop-dynamics regardless of the lock voltage.

A finite current source resistance or a series resistance between the chargepump and loop-filter, make the 'on' current into the loop-filter a function of the control voltage $V_{c}$. For low $V_{c}$, more current from the supply will flow through $R_{C P}$ than it will for high $V_{c}$. Since this current combines with $I_{\text {ideal }}$ to form the effective current into the loop-filter $I_{C P}$, it means the gain of the charge-pump $K_{C P}$ is effected by the VCO control voltage. The variation in gain $K_{C P}$ means the open-loop curve $\frac{\phi_{\text {out }}}{\phi_{i n}}$ will shift up and down depending on $V_{c}$. This changes the $0 d B$ crossing point and therefore effects the closed-loop bandwidth and potentially the phase-margin. This inconsistency is also an issue if the PLL is intended for use in modulation and demodulation applications where it can distort the information and cause out-of band spurs in the frequency spectrum.

Another source of $K_{C P}$ variation is de-saturation of the current sources. As $V_{c}$ approaches either VDD or VSS, $V_{D S}$ across the drain-source junctions inside the current-sources is reduced, and eventually they fall out of saturation and cannot continue to supply current $I_{C P}$. This results in similar 'curve-shifting' as that caused by a finite $R_{C P}$, but can be far more drastic. This is one of the main reasons why analog PLLs and DLLs are increasingly difficult to build in low-voltage CMOS where the available linear swing (the range where $K_{C P} \approx$ constant) of $V_{c}$ is reduced.

\footnotetext{
${ }^{2}$ This assumes either the absence or insignificance of a higher order pole.
} 


\section{$R_{C P}$ of the Cascaded Charge-Pumps}

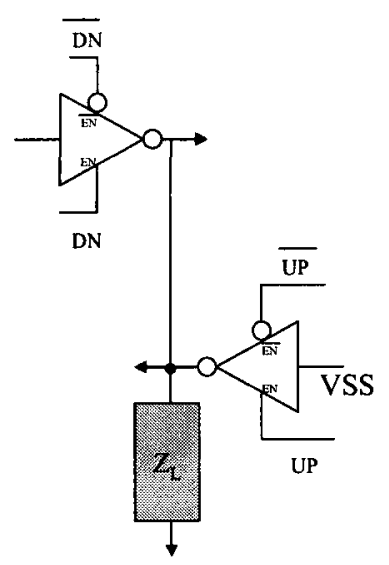

(a)

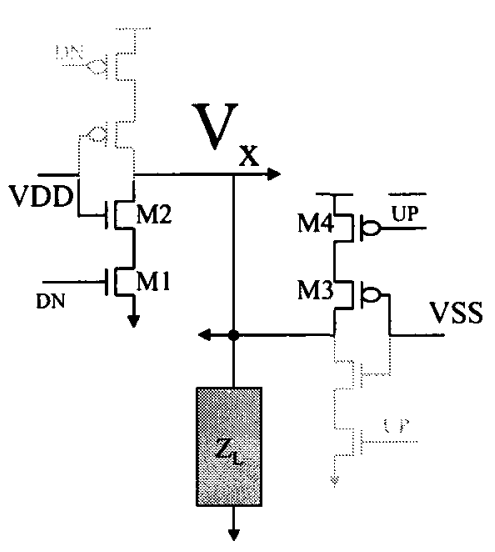

(b)

Figure 4.8: Cascaded Charge-Pump Transistor Arrangement

In the tri-state delay lines of the cascaded charge-pump, the transistors forming the charge-pump are shown in Figure 4.8. Though mid-range $V_{x}$ levels will be suitable to saturate both transistors M1 and M4, as $V_{x}$ changes one of the transistors will fall into its linear region. The charge-pump element will behave less like a current source and more like a resistive switch to VDD or VSS. Despite a single stage falling out of saturation however, the overall current sourcing/sinking ability (to be covered in the next section) maintains desirable consistency since the next stage in the chain becomes more effective, as in Figure 3.4. As an aside, in Figure 4.8, the grayed-out transistors are never used and are removed (as was covered in 4.2.2).

\subsubsection{Characterizing Charge-Pump Gain}

The cascaded charge-pump uses a cascaded set of non-ideal charge-pumps, where the charging and discharging current sources are created with the transistors inside a tri-state buffer or inverter. These non-ideal current sources contain transistors which source or sink current to the output node $V_{x}$. Depending on the input voltages, transistor sizings, and output voltage $V_{x}$, the transistors will behave more like currentsources or resistive switches. To manipulate the charge-pump current $I_{C P}$ and gain 

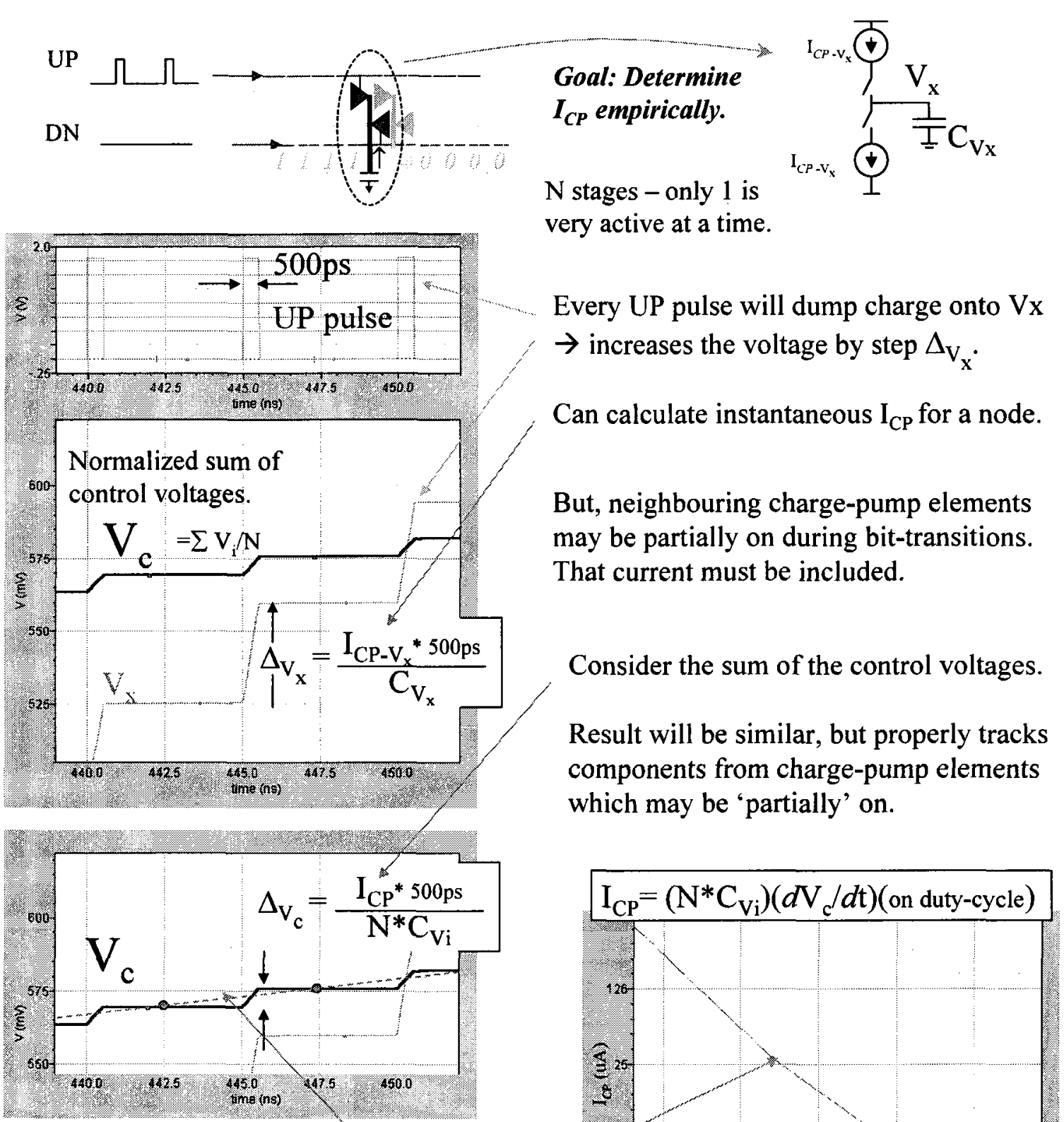

How badly does $I_{C P}$ vary with $V_{c}$ ?

$\rightarrow$ How does the step size $\Delta_{V_{c}}$ change?

This information can also be gathered from the long-term slope of $\mathrm{V}_{\mathrm{c}}: d \mathrm{~V}_{\mathrm{c}} / d \mathrm{t}$

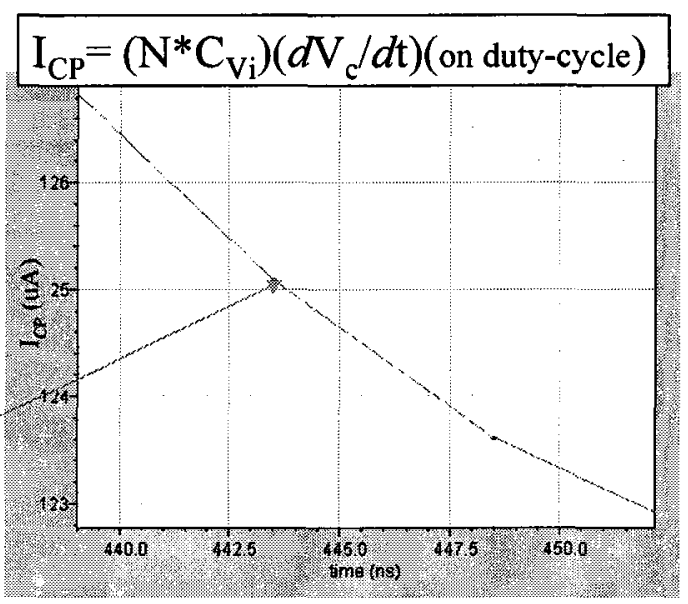

Figure 4.9: Characterizing the Gain of the Cascaded Charge Pump 
$K_{C P}$, the transconductance $\left(g_{m}\right)$ of the transistors inside the tri-state cells can be manipulated - normally through transistor sizing.

Given a particular configuration, Figures 4.9 and 4.4.2 illustrate the process for empirically determining the effective charge-pump current $I_{C P}$, and hence chargepump gain $K_{C P}$.

Within the thermometer coded cascade, most of the nodes are held at 1 or 0 and, in principle, only the charge-pump element at the transition point of the thermometer code is active. While the UP signal is asserted, current is continually sourced into the load-capacitor $C_{V x}$ at this transition point. Once the pumps are turned off, some amount of charge will have been integrated and a voltage step $\Delta V_{x}$ will have developed. Given that $I_{C P}=C_{V x} \Delta V_{x} / \Delta t$, and knowing the capacitance and duration of the UP pulse $\Delta t$, the charging current $I_{C P}$ is easily calculated.

In practice, as the transition point of the code is being swept across cells in the cascade, one charge-pump will begin to saturate and lose effectiveness while its neighbour begins to turn on and compensate. Rather than attempt to isolate the contributions of each element, the conglomerate charge-pump current can be calculated by analyzing the voltage steps on the sum of control voltages $V_{c}=\Sigma V_{i}$. Calculating the charge-pump current $I_{C P}$ from these voltage steps, $\Delta V_{c}$, takes into account the hand-off between cells. This is the technique which was used in Figure 4.9 .

\section{Cascaded Charge-Pump Consistency}

Ideally the effective charge-pump current $I_{C P}$ will remain constant as the thermometer code is swept, both within a single cell and across into neighbouring positions. A constant charge-pump current would be reflected by consistently sized voltage steps $\Delta V_{c}$ with each UP pulse. Since $I=C d V / d t$, the charge-pump current can also be obtained by taking the derivative of a sampled version of the $\Delta V_{c}$ curve, but making an allowance for the fact that the charge-pump is only on for a fraction of the entire cycle time. This is also illustrated in Figure 4.9.

Unfortunately, because of a non-linear charging characteristic and imperfect 'hand-off' as the thermometer code progresses between nodes, the total charge-pump current $I_{C P}$ will fluctuate somewhat depending on the state of the thermometer code. To capture this inconsistency, the system can be described by a minimum, average 

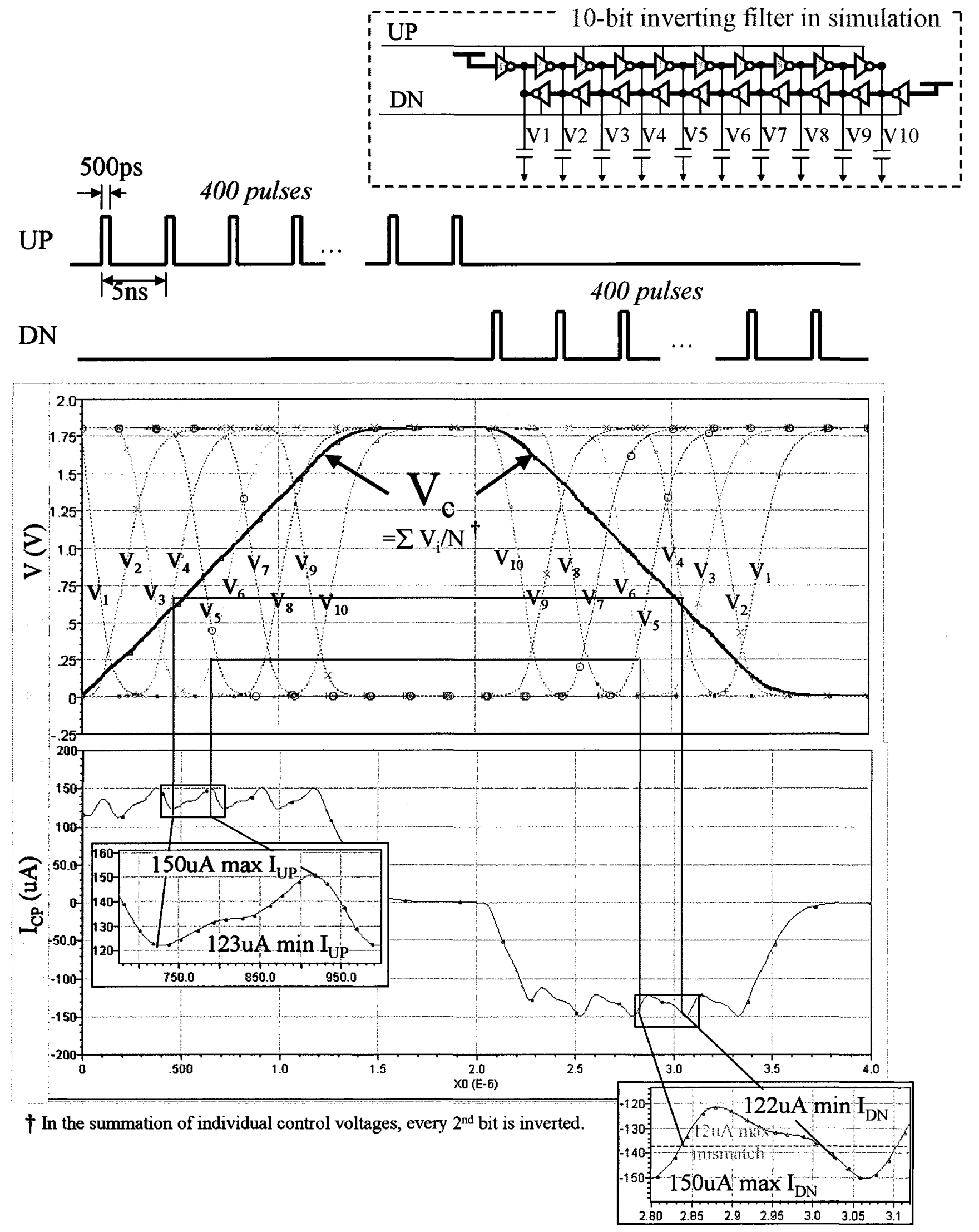

Figure 4.10: Consistency of Cascaded Charge-Pump UP and DN Currents 
and maximum $I_{C P}$ and/or $K_{C P}$. As with non-linearities in a conventional chargepump, $K_{C P}$ fluctuations will result in shifting of the open-loop curve $\frac{\phi_{o u t}}{\phi_{r e f}}$ (Figure 4.7) depending on the lock-position, and thus some measure of uncertainty in the closed-loop bandwidth $\omega_{3 d B}$.

Figure 4.10 illustrates the charging and discharging characteristics of a 10-node inverting filter. When stimulated by repeated UP pulses, the individual control voltages successively discharge (for active-low nodes) and charge (for active-high nodes). The normalized sum of these control nodes, with appropriate inversions, is also shown as the dark curve $V_{c}$. The procedure given in Figure 4.9 is used to plot the effective charge-pump current $I_{C P}$ as the thermometer code is swept. Neglecting end-effects, the charge-pump current shows remarkable consistency, varying between $123 \mathrm{uA}$ and $150 \mathrm{uA}$ (only $\pm 10 \%$ ) as one node saturates and the neigbouring node turns on. This would result in a $\pm 5 \%(\sqrt{1.1})$ fluctuation in closed-loop bandwidth. Since there is often signficant flexibility in selecting this bandwidth, in most applications such a margin would be acceptible.

An important feature of the cascaded charge-pump is that the operating frequency range, which is relatively linear with control voltage, can be extended simply by adding more stages to the cascade. This is in contrast to analog control techniques where the linear range is limited by the available vertical swing of the control voltage.

\section{UP/DN Current Mismatch}

In Figure 4.10, once the thermometer code has saturated, the UP pulses are eventually turned off and repeated DN pulses are applied to discharge the output. The chargepump current for UP and DN pulses should ideally match (but with opposite polarity). Any mismatch will result in extra current being sourced or sinked into the filter during dead-zone avoidance pulses.

As expected due to the system symmetry and the inverting code, the minimum, maximum and average DN current have the same values as the UP current. Given a maximum current of $I_{C P}=150 u A$ in one direction and minimum current of $I_{C P}=$ $123 u A$ in the other, the worst-case current mismatch would be $27 u A$. This number, however, is pessimistic. What is important is how the UP and DN currents compare at any particular lock-point, and the previous calculation assumes that both current sources are at their extreme operating points simultaneously. Instead, the peaks and 
troughs of the charging sensitivity - where $I_{C P}$ is near its maximum and minimum values - can be correlated with specific operating points. By following the flight lines in Figure 4.10 these operating points are tracked over to the discharging characteristic, where the DN current at those points can be determined. Such an analysis shows that when the UP current is at its maximum or minimum values, the DN current is near its nominal value - and vise versa. This means the worst case mismatch $(12 u A)$ is about half of that calculated by the pessimistic approach.

\subsection{Filter Stages}

Each charge-pump element (at least the 'active' ones) are coupled to a load impedance. This combination performs filtering similar to a regular charge-pump and loop-filter. The main difference is that in the cascaded charge-pump the control voltage $V_{c}$ is partitioned into $\mathrm{N}$ stages, reducing the effective VCO gain $K_{V}$ on the transient node.

As in the conventional scenario, the filtering impedance normally consists of an integrating capacitor, or an RC stage if a stabilizing zero is necessary. These two options were indicated in Figure 3.6.

\subsubsection{Integrators}

To form an integrator, as in a DLL, capacitance $C_{\text {stage }}$ is simply added to each output node of the cascaded charge-pump. The total capacitance is then $N \bullet C_{\text {stage }}$, and the loop-filter open-loop response has a $1 / s$ characteristic which shifts up or down in proportion to $\frac{K_{C P} \bullet K_{V}}{C_{\text {stage }} \bullet N}$.

To illustrate this, assume without loss of generality, that all but one node of the thermometer code is held constant at logic 1 or 0 . The single node under analog control has capacitance $C_{\text {stage }}$ which integrates current $I_{C P}$. If $C_{\text {stage }}$ is made $N \mathrm{x}$ smaller than the $C$ in a single voltage system it will fluctuate far more, but, since this single node contributes only $1 / N^{\text {th }}$ to the VCO or delay-line control, the overall effect is the same. From this perspective, one treats the system as a single-voltage one with $K_{V}$ reduced to $K_{V}^{\prime}=K_{V} / N$. This yields the expression above, and the open-loop curve $\phi_{\text {out }} / \phi_{\text {ref }}$ is offset by $\frac{K_{V}}{N} \bullet \frac{K_{C P}}{C_{\text {stage }}}$. 
If $\mathrm{N}=1$, the cascaded charge-pump simplifies into a conventional charge-pump and loop filter. If $\mathrm{N}$ is increased, for example by $20 \mathrm{x}$, the capacitance per stage $C_{\text {stage }}$ can be reduced by $20 \mathrm{x}$ while maintaining the same loop dynamics. Most nodes, however, are fixed at logic 1 or 0 , and capacitance is only required at the analog transition point of the thermometer code. This will allow the dynamic shuffling of only three $C_{\text {stage }}$ capacitances to the transition region of the code, regardless of the number of nodes $N$. This approach is useful to maintain filter dynamics but at a much lower cost in terms of area and capacitance.

Rather than reducing the capacitance $C_{\text {stage }}$ as $N$ is increased, from the expression $\frac{K_{V}}{N} \bullet \frac{K_{C P}}{C_{s t a g e}}$ it follows that if $C_{\text {stage }}$ is kept constant, $K_{C P}$ can be increased while $N$ is increased with no effect on loop dynamics. This trades off charge-pump gain for $\mathrm{VCO} /$ delay-line gain $\left(K_{V} /\right.$ node $)$, and as covered in Section 3.7, can improve reference referred noise suppression.

\subsubsection{Moving $\omega_{p 1}>0$}

To form a low-pass filter, as desired in Type I PLLs, an extra resistance is effectively placed in series between each charge-pump stage and its output load $C_{\text {stage }}$. Due to the non-ideal nature of the charge-pump elements, some natural resistance already exists but this can be further exploited through transistor sizing, bias arrangements and the addition of further devices (eg. transistors biased in the linear region) to move this pole further out.

\subsubsection{Implementing a stabilizing zero $\omega_{z}$ - Type II PLLs}

In the previous discussion, it was argued that increasing from a single voltage system to an $\mathrm{N}$-node cascaded charge-pump allows the capacitance/stage to be reduced from $C$ to $C / N$ without effecting the loop dynamics. This was true since the vertical offset of the open-loop transfer function in an integrator uniquely defines the $0 \mathrm{~dB}$ crossing point and hence the characteristics in the closed-loop system. In standard (Type II) PLL configurations, however, a stabilizing zero is necessary to ensure phase-margin and loop stability. 
Effect of partitioning the control voltage in the thermometer filter

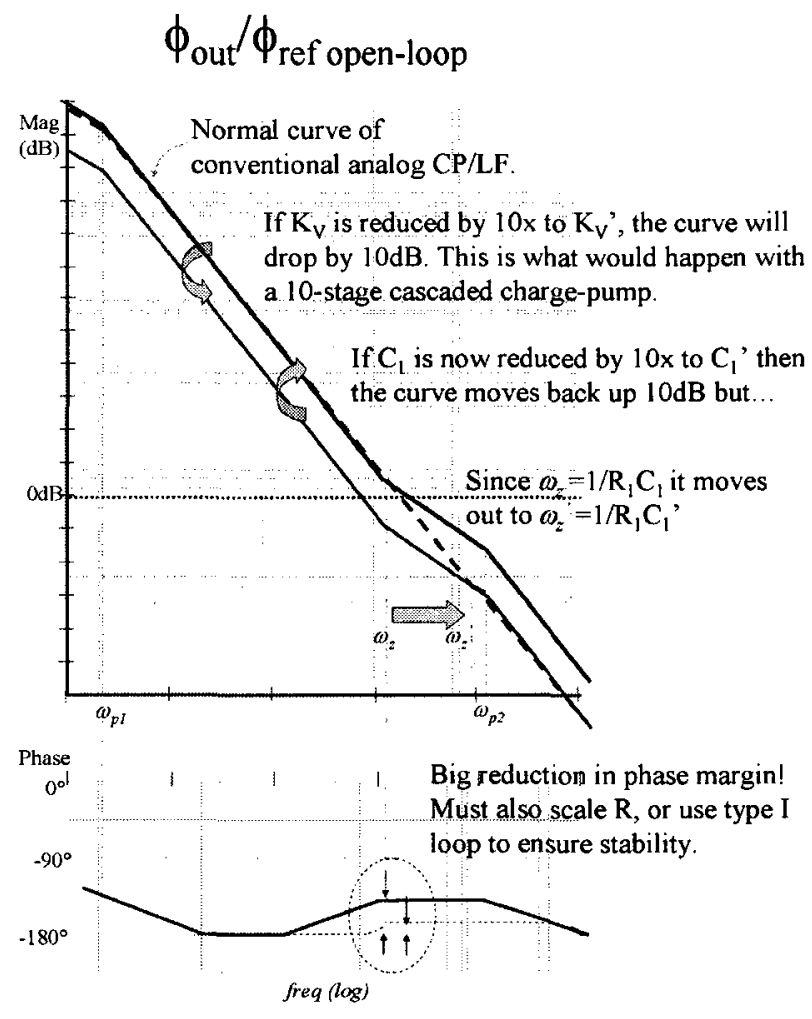

(a)
Effect of increasing charge-pump gain

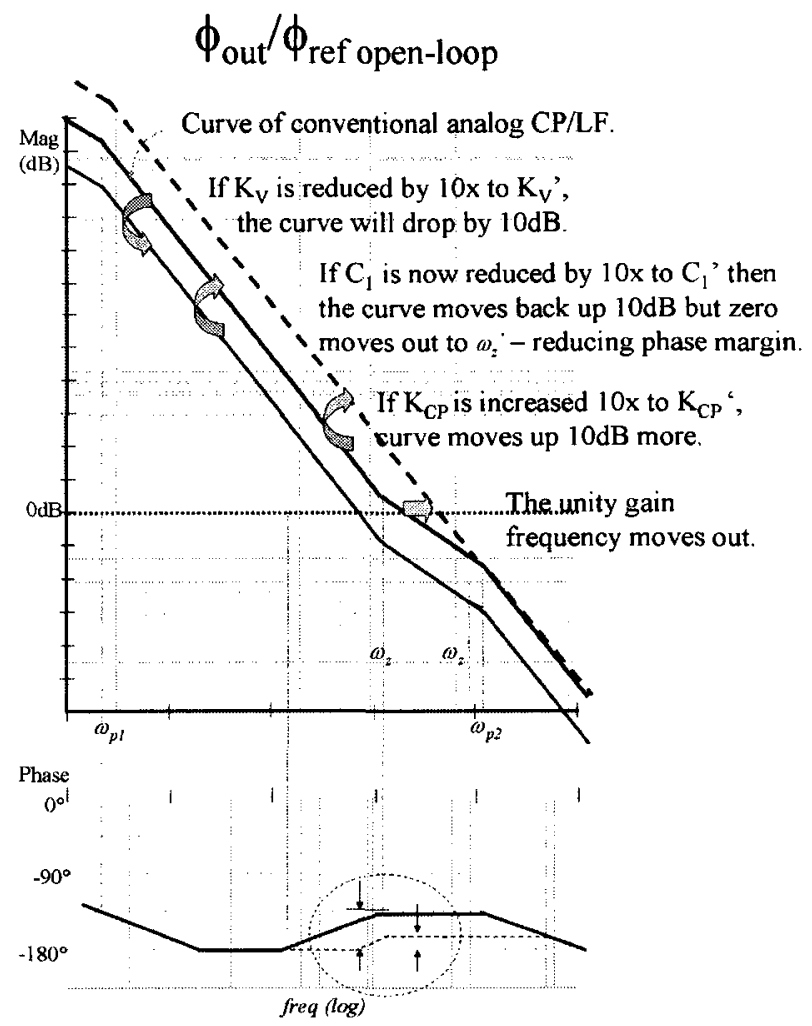

(b)

Figure 4.11: Loop Effects of partitioning the VCO control in Type II PLLs

Figure 4.11a illustrates the effect of introducing a 10-node thermometer code into a normal analog loop with integration capacitor $C_{1}$ and $\omega_{z}=1 / R_{1} C_{1}$. Adding 10nodes of control reduces the effective VCO gain by $10 \mathrm{x}$, shifting the curve downwards. Reducing the capacitance on each node from $C_{1}$ to $C_{1} / 10$ then shifts the curve back up, but, since the zero is located at $\omega_{z}=1 / R_{1} C_{1}$, it will move out to $\omega_{z}^{\prime}=N / R_{1} C_{1}$, potentially reducing phase-margin. To keep the zero in place, it is important to increase $R_{1}$ with any decrease to $C_{1}$.

\subsection{Sharing Filter Sections}

In the analog thermometer code, only one or two stages are ever undergoing analog transitions at a time. All of the other stages are pinned at either 0 or 1 , and any 


\section{$1^{\mathrm{UP}} 1111 \sim 0000 \div$}

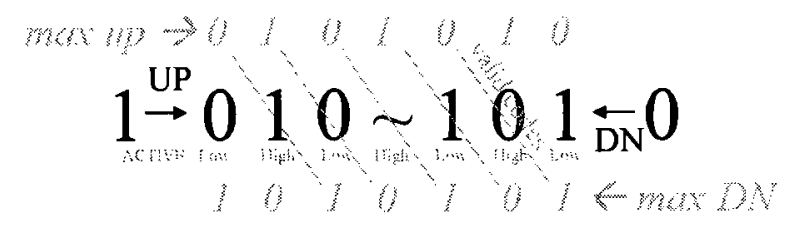

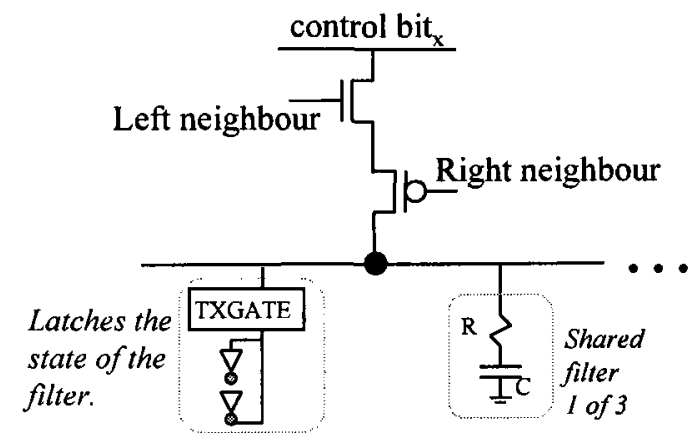

(a) Non-Inverting Code

Need to use transmission gates for a strong connection to the filter.

Get inverting control from extreme neighbours.

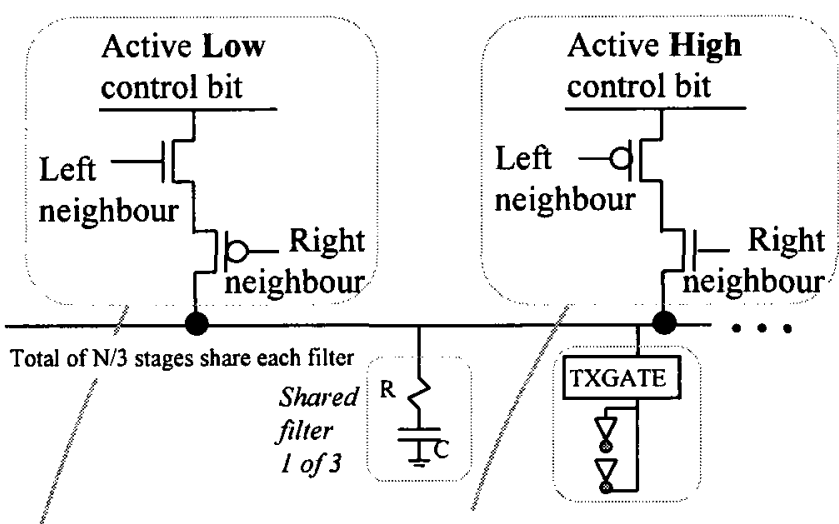

(b) Inverting Code

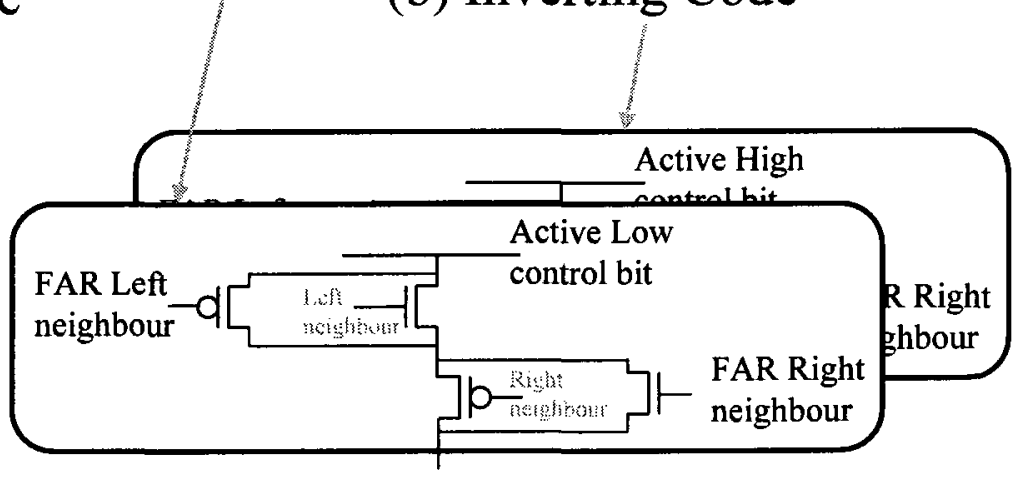

\section{(c) Inverting Code with Transmission Gates}

Figure 4.12: Logic for Connecting Shared Filter Sections and State-Retention latches to the Code's Transition Point: Transmission gate logic examines neighbouring nodes to determine the transition point of the code and, if under contention, connect to a shared filter section. 
filtering impedances attached to their nodes is unused. This creates the opportunity to share hardware. The task merely becomes connecting the shared filter sections to the analog transition region of the code.

To illustrate how this switching is performed, assume for the moment that only one node can maintain an analog voltage - and all others are at 0 or 1 . As shown in Figure 4.12, logic at each position must check to see whether it is the node at the transition point of the code, and if it is, connect to the filter.

In the case of a non-inverting code, shown in Figure 4.12a, logic at each position checks to see if its neighbours disagree. ${ }^{3}$ If they do, that control node is the transition point and should be connected to a filter.

For the inverting code in Figure 4.12b it follows the same principle. Logic at each node checks its neighbours to see if it is the point of contention. In this case the logic network is slightly different depending on whether the node in question is active-high or active-low. In either case though, it is looking for the condition where its neighbours disagree, being either $1 x 0$ or $0 x 1$. Since it is supposed to be an inverting code, these patterns are inconsistent (ie. only 101 or 010 are valid) and indicate that the node in the middle is the transition point of the code and should be connected to a filter.

Using PMOS and NMOS pass transistors in the configurations of Figures 4.12a and $4.12 \mathrm{~b}$, though logically correct, performs poorly. Since PMOS switches don't conduct low voltages and NMOS switches don't conduct high voltages, using them in series means the switch only works at mid-range levels. To solve this problem, a conventional solution is to implement a transmission gate, rather than a simple pass transistor. To control it however, an inverted version of each neighbour is required, and since the values may be analog in nature, they should not be fed into a CMOS inverter. To solve the problem, one can note that by virtue of the inverting thermometer code, we also have access to the inverted versions of our left and right neighbours by looking out one stage further on each side. Complementary NMOS and PMOS transistors are therefore added into the switch logic to form transmission gates, and then these inverted signals from the extreme neighbours are used as their control inputs. This improved configuration is shown in Figure 4.12c.

\footnotetext{
${ }^{3}$ Since the thermometer code is only valid in one direction, it only needs to check the $1 x 0 \mathrm{com}$ bination, and not $0 x 1$.
} 
In this scenario, we share 3 filter-units (either capacitors $C$ for Type I PLLs and DLLs, or $R C$ filter stages in the case of Type II PLLs) between all $\mathrm{N}$ stages of the cascaded charge-pump. Sharing 3 stages is important in practical scenarios since up to 2 control nodes may be undergoing analog transitions at any time, and we use an odd number of stages to prevent problems when switching discharged filters onto charged control nets, and vise-versa. Measured results, showing how this rotation takes place, will later be shown in Figure 5.9.

Rather than use fixed values for $R$ and $C$, it is often desirable to make these adjustable. The effective value of $R$ can be modified by changing the sizes of the switches in the logic network, or by implementing $\mathrm{R}$ with active devices. Similarly, $C$ can be made using a varactor, switched capacitances, or a combination. Finally, the shared filter section can be made using most other active or passive filtering techniques.

\subsubsection{Effective Capacitance Multiplication}

As has been previously discussed, each stage of the cascaded charge-pump requires a capacitance of $C / N$ to maintain the same loop dynamics as an analog filter with capacitance $C$. Capacitances are typically the dominant area cost in analog PLLs and DLLs. Because of the dynamic filter rotation, only 3 small capacitances of $C / N$ are required, regardless of the number of thermometer stages.

Furthermore, because of the dielectric leakage insensitivity of the cascaded charge-pump (to be discussed in Section 4.8), area efficient MOS capacitors can be used, rather than MiM capacitors, metal-to-metal traces or off-chip components.

As one example of these savings, the PLL to be considered in Chapter 5 has an effective capacitance of $60 \mathrm{pF}$ integrated on chip using only $3 \mathrm{pF}$ of capacitance. Along with the transmission gate switches which allow for adjustable bandwidth, the total area of the switched capacitances consume 304 equivalent gates of area or $3,708 \mu \mathrm{m}^{2}$. To implement a single, unadjustable, $60 \mathrm{pF}$ capacitance with MiM capacitors in the same technology (TSMC $0.18 \mu \mathrm{m}$ ) would require at least $57,600 \mu \mathrm{m}^{2}$. 


\section{Smoothing capacitance $C_{2}$}

In most analog filters an additional high frequency pole is created on the VCO control node with a small smoothing capacitor $C_{2}$. This is necessary to reduce the effects of sampling ripple on $V_{c}$. In the cascaded charge-pump, its size can also be scaled by $1 / N^{\text {th }}$ that of the analog case, and so it can be implemented with either the inherent parasitic capacitance of the node, or with an additional MOS capacitor.

\subsection{Stabilizing the Digital Values}

Since the UP and DN currents in the cascaded charge-pump are not always matched, efforts will be made to eliminate or reduce the width of dead-zone avoidance pulses. Since tri-state elements are used to build the cascaded charge-pump, when there is no activity on the UP or DN signals (as in ideal lock), then the control nets are unconnected. During this time, their capacitances would ideally hold their charge and maintain the thermometer coded state. For a number of practical reasons, the voltages on these capacitances may leak and/or fluctuate due to noise and coupling.

The thermometer string can potentially be made more stable by connecting those voltages which have already hit their limit to a reference (normally VDD/VSS or clean versions thereof) as appropriate. This removes their susceptibility to leakage, and lowers their response to coupled noise sources. This is also a requirement if one intends to recycle passive components as advocated in the previous section.

Performing this digital stabilization is made relatively simple due to the nature of the thermometer code. Simple logic at each position can look at its neighbors to determine whether the transition point of the code has already passed-by. If it has, the node should be tied-off, otherwise it should be left to undergo analog control.

This is illustrated in Figure 4.13a for a non-inverting code ${ }^{4}$ and Figure $4.13 \mathrm{~b}$ for the more efficient inverting configuration. Only 2 transistors need to be added per control node to perform the necessary check and tie-off.

Directly using the method depicted in Figure 4.13b has an unfortunate sideeffect, but one which can be easily cured. According to the natural behaviour of the inverting filter, as one node charges past $\approx \mathrm{VDD} / 2$ the neighbouring node begins to

\footnotetext{
${ }^{4}$ In this case the tie-off would be poor because of the threshold drop when using NMOS pull-ups and PMOS pull-downs.
} 


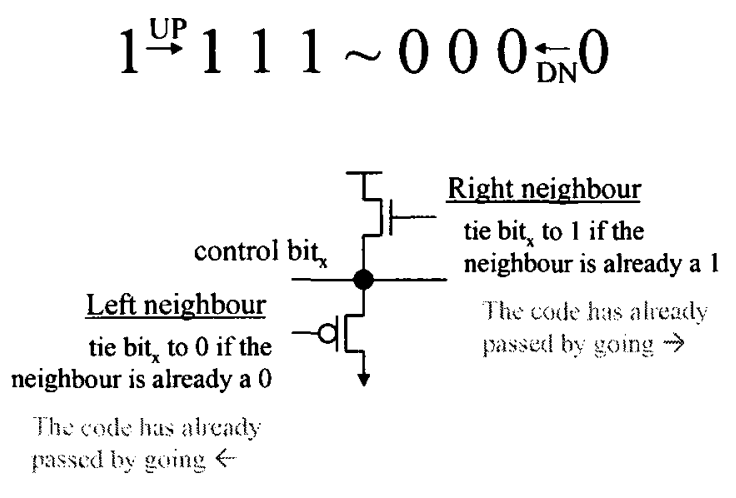

(a) Non-Inverting Code

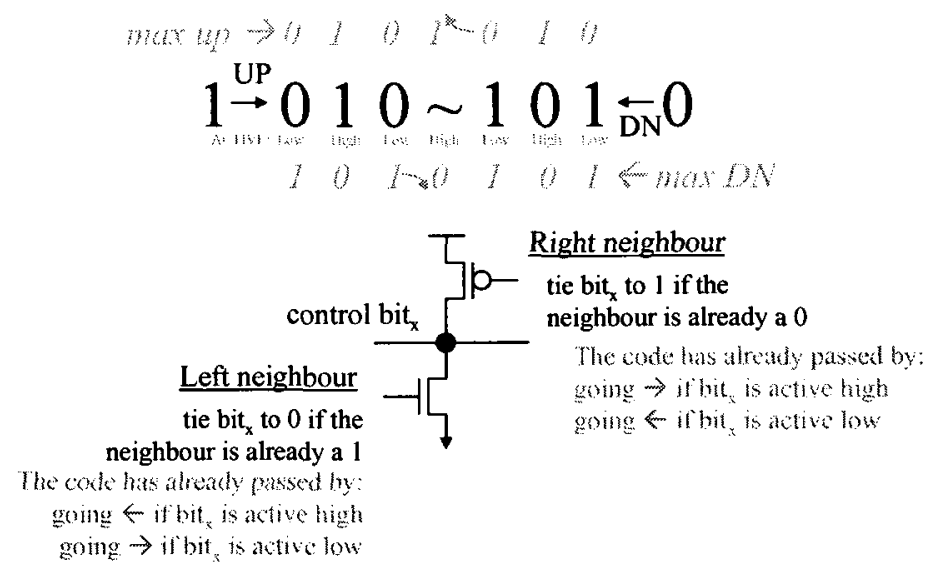

(b) Inverting Code

Figure 4.13: Digital Stabilization Logic to tie-off saturated nodes to VDD/VSS

discharge. This overlap is responsible for the gradual 'hand-off' of the transition point between nodes (as studied in Section 4.4.2). When using the tie-off logic in Figure $4.13 \mathrm{~b}$, once the neighbour discharges enough it will kick-in the bypass transistor, and the positive feedback accelerates the charging of the original node and 'snaps' it to logic 1 . The same occurs near logic 0 . This may result is regions of instability, where the system cannot properly accommodate lock-points that call for analog voltages near the supply rails. The simple solution is to look at a neighbour 2 positions away, rather than the immediate neighbour.

\subsection{Leakage Sensitivity}

In a cascaded charge-pump the majority of VCO control nodes are tied off to logic 1 or 0 . Since these nodes are not in a high-impedance state, they are not susceptible to leakage. It is interesting, however, to examine the effects of leakage on the analog node(s) at the code's transition point. In normal implementations of an $N$-node cascaded charge-pump, an effective capacitor of $C_{1} / N$ will be connected to each node (where $C_{1}$ represents the size of the required capacitance in a conventional singlevoltage filter). Figure 4.14 illustrates how leakage effects compare in these two cases. 


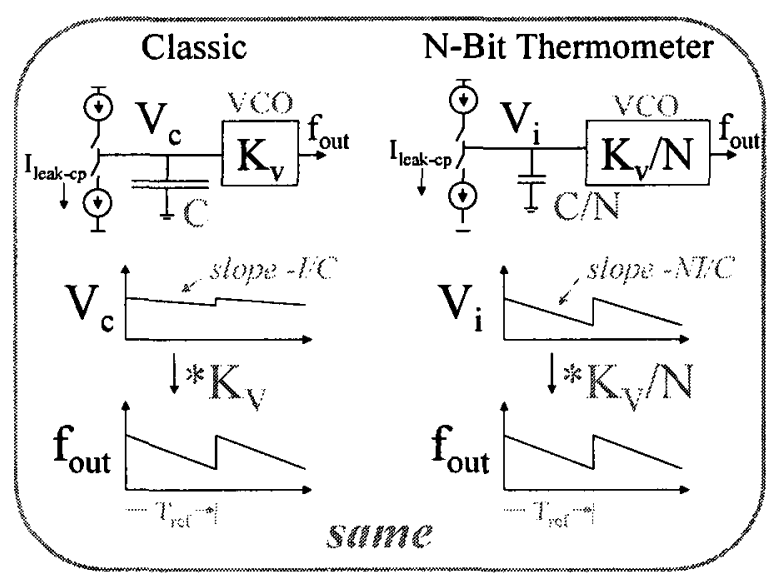

(a) Charge Pump Leakage

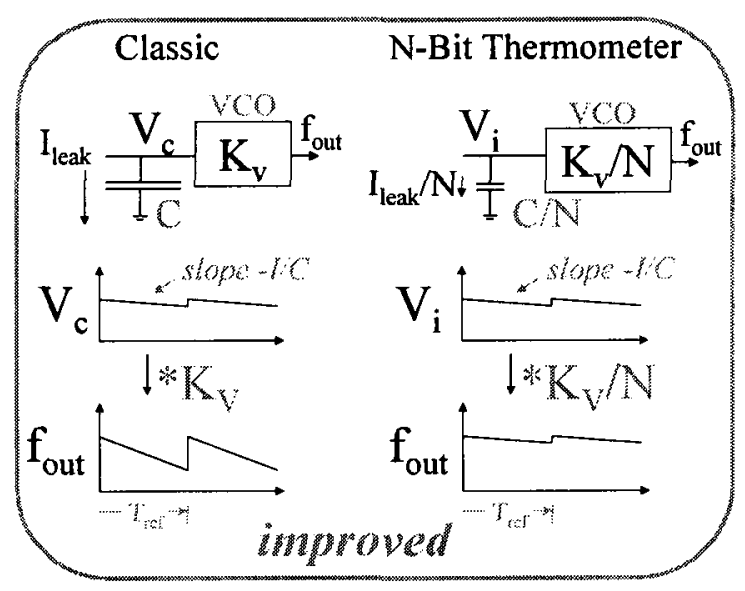

(b) Dielectric Leakage

Figure 4.14: cascaded charge-pump Leakage: Charge-pump leakage has the same effect as in a conventional system, but dielectric leakage effects are reduced by $\approx N \mathrm{x}$.

\subsubsection{Charge-Pump Leakage}

Assuming a charge-pump element of similar construction, the leakage current in both cases will be identical. In the cascaded charge-pump, since the capacitance is $1 / N^{\text {th }}$ the size, the control voltage will drop much faster, but since this contributes little to the overall $\mathrm{VCO}$ frequency $\left(K_{V}^{\prime}=K_{V} / N\right)$, the resultant frequency deviation is equivalent in both cases.

\subsubsection{Reduced Effects of Dielectric Leakage}

Since dielectric leakage current is proportional to capacitor size, the leakage induced voltage drop on a small capacitor and big capacitor will be roughly identical. In the case of the cascaded charge-pump however, this drop is scaled by a relatively low VCO gain $\left(K_{V} / N\right)$ compared to a single-voltage system. As a result, dielectric leakage will cause frequency disturbances which are reduced by $\approx N \mathrm{x}$ compared to a conventional analog system. This compensation permits the use of the very area efficient (but 'leaky') thin-oxide MOS capacitors. Not only does this reduce space and congestion in the layout, but it permits the use of exclusively digital processes (without the analog MiM option) for reduced fabrication costs. 


\subsection{Supply Noise Sensitivity}

If the majority of control voltages are digitally restrained at VDD or VSS, supply sensitivity becomes an immediate concern. Supply noise can be a dominant source of error for analog circuits in digital environments. Fortunately though, there are helpful conditions which mitigate the effects of supply noise.

\subsubsection{Varactor Sensitivity}

If the cascaded charge-pump outputs control delay elements using MOS varactors, which is the most likely approach, then they are relatively insensitive to noise near either supply rail. This is illustrated with Figure 4.15, taken from [28], where the flat regions of the CV curve fortunately correspond to control voltages near VDD and VSS. Fluctuations of the control voltages around these points have little effect on the load capacitance, and so supply sensitivity is very low.

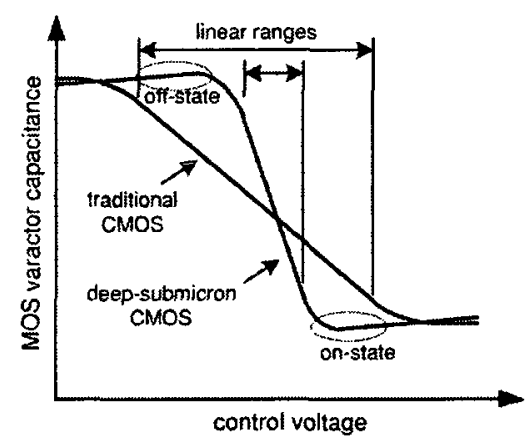

Figure 4.15: MOS varactor CV characteristic [28]

\subsubsection{Switch Sensitivity}

If the control string is used to manipulate the $g_{m}$ of loading switches, rather than as varactor bias levels, then the switches are insensitive to changes while they are in the OFF state: below $V_{t h}$ for NMOS transistors, and above VDD - $V_{t h}$ for PMOS transistors. If they are ON (VDD for NMOS, VSS for PMOS), then any delay induced due to supply/ground noise on the control lines, opposes the natural speed change of the driving elements. For example, if $\operatorname{VDD} \uparrow$, the drivers in the delay-line will speed 
up, but the NMOS switches which are ON will become stronger, exposing more capacitance, and thus countering the increased driver strength. The same example applies to ground bounce and PMOS switches. Through careful modeling and sizing, the $+v e$ and $-v e$ effects can be tuned to cancel each other out at a particular setting of the control string (eg. the middle of the tuning range), yielding (ideally) zero supply sensitivity. Though tuning to ensure this exact cancellation would be burdensome if not impractical across corners, the negative correlation is a very fortunate benefit nevertheless.

\subsubsection{Supply Filtering}

It should also be noted that a low-pass filter exists between VDD/GND and the control nodes. The tie-off transistors (Figure 4.13) in combination with the capacitance of the output node form a low-pass filter which has a BW that can be adjusted through sizing. Typical values might be $g_{m} / C=(100 f F * 100 k)^{-1}=100 \mathrm{MHz}$. Though this is well above the loop-BW, it helps to reject any high frequency transients on the supply which would otherwise alias in near the carrier.

As a separate issue, supply noise which influences the VCO or delay-line is subjected to the loop-dynamics as though it originated in the VCO. As such, the loop suppresses it within the loop-BW, as shown in Figure 2.6.

\subsection{Phase Detector Conditioning}

The output from a conventional phase/frequency detector (PFD) can be used to directly feed the cascaded charge-pump. Various improvements may be possible however, by preconditioning the PFD outputs before reaching the cascaded charge-pump's control ports. The primary motivation for these stages is to manipulate the gain and dynamic response of the cascaded charge-pump at little expense.

A preview of the various preconditioning options is shown in Figure 4.16. Any of the elements in the chain are optional, and they each have advantages and disadvantages. It should also be noted that the cascaded charge-pump requires 4 control inputs: $U P, D N$, and the inverted versions $\overline{U P}$ and $\overline{D N}$. If preconditioning is used, 


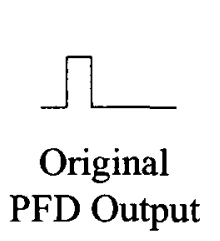

(a)
Optional pre-processing stages

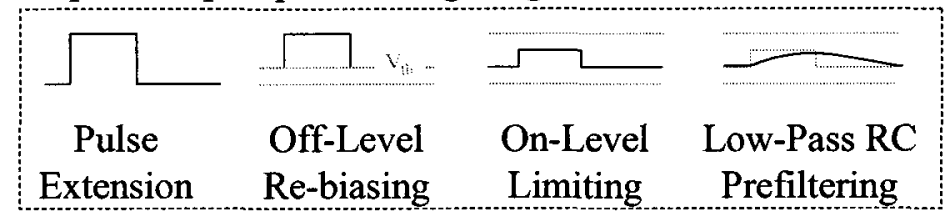

(e) control to thermometer filter

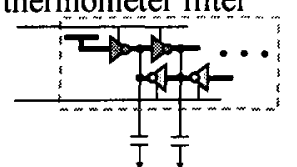

(f)

Figure 4.16: Optional Preconditioning between the PFD and cascaded charge-pump each control signal should go through similar stages, and so 4 sets of these circuits are necessary.

First, the rationale for each stage will be discussed before proposing some efficient circuits to perform the various chores.

\subsubsection{Preconditioning Rationale}

\section{Pulse Extension for $K_{C P}$ Manipulation (Figure 4.16b)}

Conventionally, charge-pump gain $K_{C P}$ is controlled by increasing the charge-pump current $I_{C P}$. Unfortunately, in a typical charge-pump, the peak current is forced into the loop-filter during any phase correction and this causes spikes on the VCO control voltage. These spikes are proportional to the peak current. These spikes also force the loop-BW to be lower than 10x the reference frequency to maintain the validity of the continuous time approximation. If, rather than force more peak-current into the loop in sharp spikes, the charge-pumps are left on for a longer duration, the magnitude of the spikes will be reduced.

\section{Logic Off Re-biasing for Faster Response (Figure 4.16c)}

Normally the phase-frequency detector drives the gates of the charge-pump switches completely from VSS to VDD and then back down from VDD to VSS. While the control signal is being charged from VSS through to $V_{t h}$, there is very little change in conductivity of the charge-pump, but it nonetheless consumes time and power to charge the PFD output load up to $V_{t h}$. If, instead of discharging the control voltage all the way off to VSS, the charge-pump only pulled the voltages off to $V_{t h}$ then, on the following cycle, the PFD output load will be slightly precharged, and both the PFD 
and charge-pump can react quickly. In fact, transistors biased at $V_{t h}$ are operating at the border of the subthreshold region, where their gain is exponential with $V_{g s}$, [17] making them very sensitive to even small phase-errors. A further advantage of this approach, particularly in a large cascaded charge-pump where the capacitive loading on the control port may be quite high, is the reduced voltage swing that occurs with every update cycle. This can significantly reduce power consumption, and also alleviates signal feed-through problems to the VCO control line $V_{c}$. A disadvantage of this approach, is that if UP and DN leakage currents in the buffer/inverter charge-pump structures are not matched, the reduced 'off' levels will exacerbate that problem.

\section{Logic ON Limiting for $K_{C P}$ and $R_{C P}$ Manipulation (Figure 4.16d)}

The UP/DN signals from the phase detector drive NMOS and PMOS transistors in the cascaded charge-pump. Referring back to the cascaded charge-pump's charge-pump arrangement in Figure 4.8, reducing the ON voltage levels reduces $V_{g s}$ on M1 and M4 and has two main effects. First, and most obvious, it will reduce the charge-pump current, and hence charge-pump gain $K_{C P}$. The gain can be scaled back up again through suitable transistor sizing. The second effect, however, is more interesting. Transistors M1 and M4 remain in saturation (and behave like a 'good' current source) provided that $V_{d s}$ (which is $\approx V_{x}$ ) is $>V_{g s}-V_{t h}$. With full strength 'ON' pulses, $V_{g s}$ is large and there is not a wide range of values for $V_{x}$ where the current sources maintain a high output resistance $R_{C P}$. If $V_{g s}$ is reduced by a threshold voltage, this also increases the range of $V_{x}$ values for which transistors M1 and M4 remain saturated.

Limiting the on voltage to the cascaded charge-pump control ports also has the same two additional benefits that were encountered with the re-biased off level. That is, the lower voltage swing reduces power consumption and signal feed-through to the VCO control line.

\section{Prefiltering (Figure 4.16e)}

There will naturally be some capacitive load on the input ports of the cascaded charge-pump. Rather than repeatedly force these ports to VDD and VSS with a low resistance source, as would be done when driven directly be a digital PFD, the 
capacitance can be taken advantage of to introduce a high frequency pole above the loop-bandwidth. Provided it is at a frequency $>10 \mathrm{x}$ the expected closed-loop bandwidth, it should not effect stability but can still have a beneficial impact on reference spurs and other noise sources.

Another benefit of this prefiltering is that it will tend to lower the peak and average voltage $V_{g s}$ applied to the charge-pump's transistors M1 and M4 in Figure 4.8. As discussed in the previous section, reducing $V_{g s}$ will lead to current-sources which can support a wider range of output voltages while remaining in saturation. Since the duty cycle of the UP/DN waveforms is very short, the average value is very close to the 'off' level, and with even moderate filtering, there should not be drastic movements which form peaks on $V_{g s}$ and pull the current sources out of saturation.

\subsubsection{Implementing the Preconditioning Circuitry Pulse Extension and Off-Level Rebiasing}

Quickly opens the current tap when asked, but slowly turns it off.

Rather than increase current, increase the time its on for. Less disruptive.

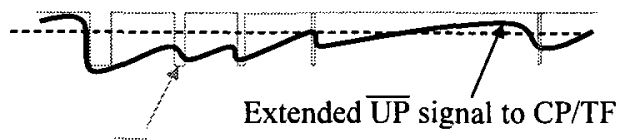

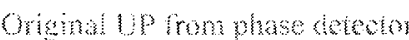
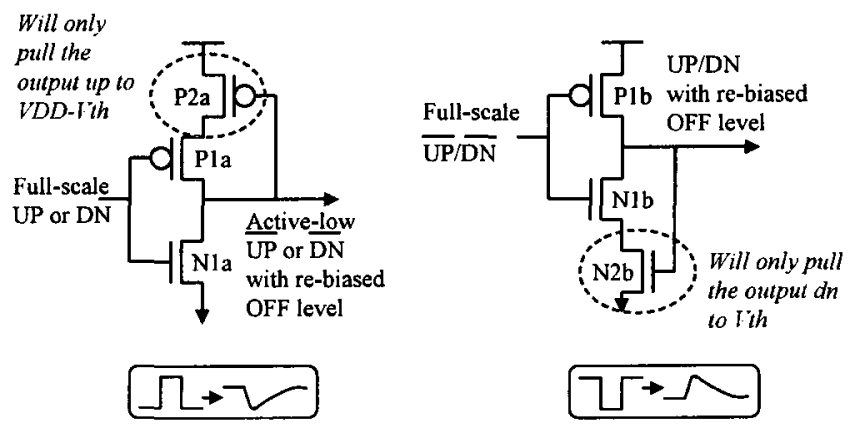

Figure 4.17: Pulse Extension and Off-Level Rebiasing Circuits (see Figure 4.16b,c)

Though this re-biasing can be performed in a number of ways, a simple option is shown in Figure 4.17. The circuits shown turn on quickly but turn off very slowly. The turn-on path is through a strong switch transistor with low on-resistance (N1a and P1b). In contrast, the turn-off path goes through a weak and increasingly starved transistor ( $\mathrm{P} 2 \mathrm{a}$ and $\mathrm{N} 2 \mathrm{~b}$ ), and therefore has a long decay time. The discharging stops as the output approaches $V_{t h}$, and so these circuits also perform off-level rebiasing. The asymmetric charging and discharging characteristic extends the PFD pulses in the time domain. Short up or down pulses are, in essence, amplified. Rather than increase 
charge-pump gain $K_{C P}$ by increasing the current, this circuit extends the control pulse to leave the current on longer. Simulations, shown in the next chapter, reveal that this pre-emphasis technique drastically increases the charge-pump response to small phase errors $($ by $\approx 6 \mathrm{x})$. Since this approach has very little effect on naturally wider phase-error pulses (it does not emphasize them as much), it creates a non-linear charge vs phase characteristic. In integer mode synthesisers, phase errors are very small and non-linearity is not an issue, making the $K_{C P}$ improvements for small phase errors a significant advantage.

\section{ON Voltage Limiters}

Shown in Figure 4.18, pass transistors can be used to easily reduce the ON voltage levels of the control pulses. Active-high pulses are fed through NMOS pass transistors - which cannot pass signals above VDD- $V_{t h}$. Similarly, PMOS pass-transistor can be used to limit the ON voltages to $V_{t h}$ (rather than VSS) in active-low signals.

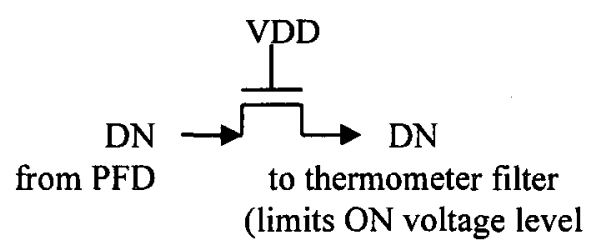

to VDD-Vth)

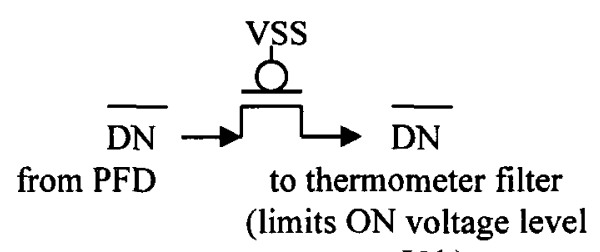

to Vth)

Figure 4.18: Using pass-transistors to limit ON voltage levels (see Figure 4.16d)

\section{Manipulating the Prefilter Pole}

Due to the inherent resistance and capacitance in the re-biasing circuits of Figures 4.17 and 4.18 , they perform some filtering of the UP/DN control before reaching the cascaded charge-pump. The level and characteristics of the filtering performed by these circuits can be manipulated by adjusting the various transistor sizes, but typically they perform fast enough that their corners are at very high frequencies and don't negatively effect stability.

Further RC adjustment can be done with a flexible transmission gate network as shown in Figure 4.19. This approach can be used to adjust the higher order pole, or to implement a zero. To preserve stability these poles (or zeros) must be taken 
Optional Steering Logic to reduce $\mathrm{C}$ Saves Power if not using $C$ for Extra Filter Pole

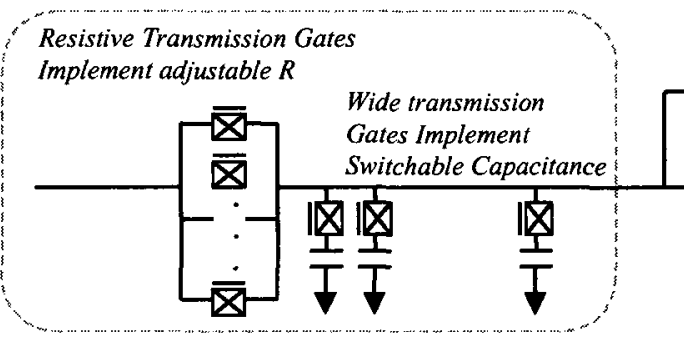

Optional Extra Variable RC filtering Note: The adjustable RC configuration is also useful for the main $R_{1}{ }^{*} C_{l}$ filter stages shared between the thermometer sections.

Figure 4.19: Adjustable RC Prefiltering and Steering Logic (see Figure 4.16e)

into account, or should be placed at high enough frequencies to ensure they do not effect the system's phase-margin.

\section{Steering Logic to Save Power}

In the cascaded charge-pump, only a few nets are under analog control at any time. The others are digitally locked at 1 or 0 . Because of the characteristics of the thermometer code, it is very easy to partition the filter into small sections and, with simple logic, steer the control to only the analog section of the cascaded charge-pump which needs it (Figure 4.19.) If the load-capacitance is not used for prefiltering, this approach can be used to reduce the loading and hence power consumption. This steering logic is particularly helpful to reduce power if a large number of thermometer stages are used, and they are being driven directly by a digital PFD.

\subsection{Saving/Recalling closest digital state}

The state of the cascaded charge-pump is approximated by the closest digital representation of the control string. The obvious way to save and hold this approximate state would be to enable a latch on each stage of the control string. This, however, adds at least 6 transistors/stage and potentially doubles the active hardware requirements. If the aforementioned techniques are used to stabilize the digital states, and 
switch non digital values to shared filter sections, a more efficient method can be used. The digital stabilization method inter-locks each net which is further than 1 node away from the analog region of the thermometer string. Those nodes are actively tied to 1 or 0 based on an analysis of their neighbours to determine which side of the code's transition point they are on. Those nodes near the analog region of the string, are instead tied to the shared filter sections. To save all the nodes of the string, it is therefore sufficient to latch only the values at the shared filters (the latches are shown Figure 4.12), which in turn locks the rest of the line. To permit operation again, the latches in the analog section are disabled, and the system recovers from the closest digital approximation of the lock state.

\subsection{Lock Position Initialization}

In addition to the ability to save and recall the filter state with minimal overhead (3 latches), it is also feasible to force particular values onto the control nodes from some external circuitry. Conceivably, a table (likely binary coded) can be used to store approximate lock codes versus frequency, and along with minimal interpolation this can be used to initialize the thermometer string to significantly speed up acquisition times.

\subsection{Summary}

Chapter 3 introduced the system level cascaded charge-pump and its benefits (reduced $K_{V C O}$ and hence better noise suppression and smaller loop filters).

Here in Chapter 4 it was shown that the circuit is built with, essentially, a simple cascade of tri-state inverters. In this structure, the current steering switch is implemented naturally, leading to the consistent injection of charge, seen in Figure 4.10 , as the analog control node is swept from cell to cell.

Since some of the control nodes maintain analog levels, it is a challenge to build logic circuits around the structure while preventing abrupt switching positions and short-circuit current problems. These problems were solved by appropriate use of transmission gate logic and the properties of the thermometer coded control to find 
the analog transition region of the code. This information, is used to rotate the loop filter to the appropriate control node with a soft-handoff approach.

The chapter has also discussed a number of other details, including supply and leakage sensitivity, gain control through PFD and CP bias circuitry, and lock-state retention and initialization. 


\section{Chapter 5}

\section{PLL Example: Simulation and Measurement}

\subsection{Introduction}

Two mixed-signal ICs were designed and manufactured to evaluate variants of the cascaded charge-pump. The die-micrographs of these ICs are shown in Figure 5.1. This chapter will focus on the simulated and measured performance of a particular x8/x32 PLL circuit on the second-die.

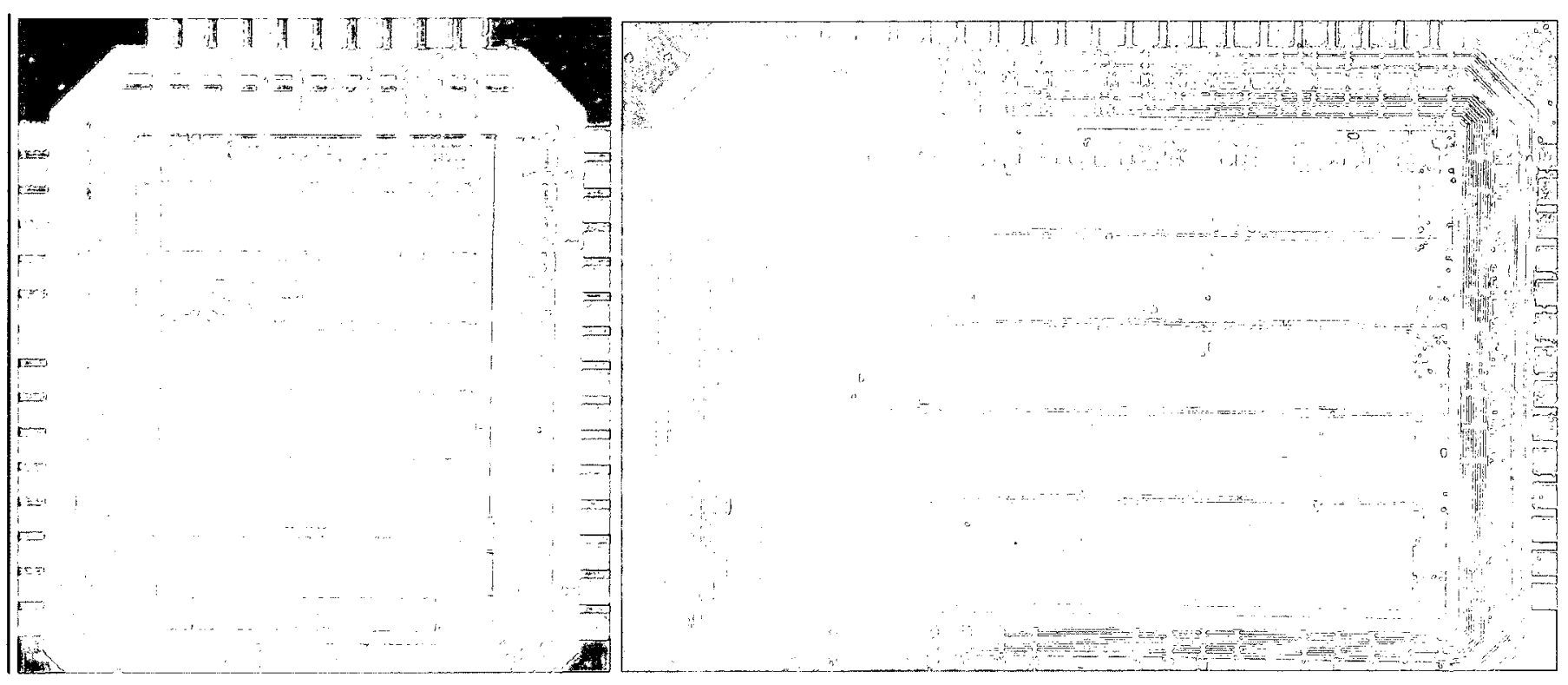

Figure 5.1: Die micro-graphs of $1^{\text {st }}$ and $2^{\text {nd }}$ prototypes. 


\subsubsection{Debug, Test Structures and Other Circuitry}

In addition to the circuit to be discussed in this chapter, the die contained other PLLs and DLLs, and a general purpose testbed to mix-and-match various synthesizer components. A block diagram of the die is shown in Figure 5.2. Circuits were also added for observation and control of the various components. A graphical-userinterface was developed to organize the control and read the status of the device. A screenshot of the software, with annotations, is shown in Figure 5.3.

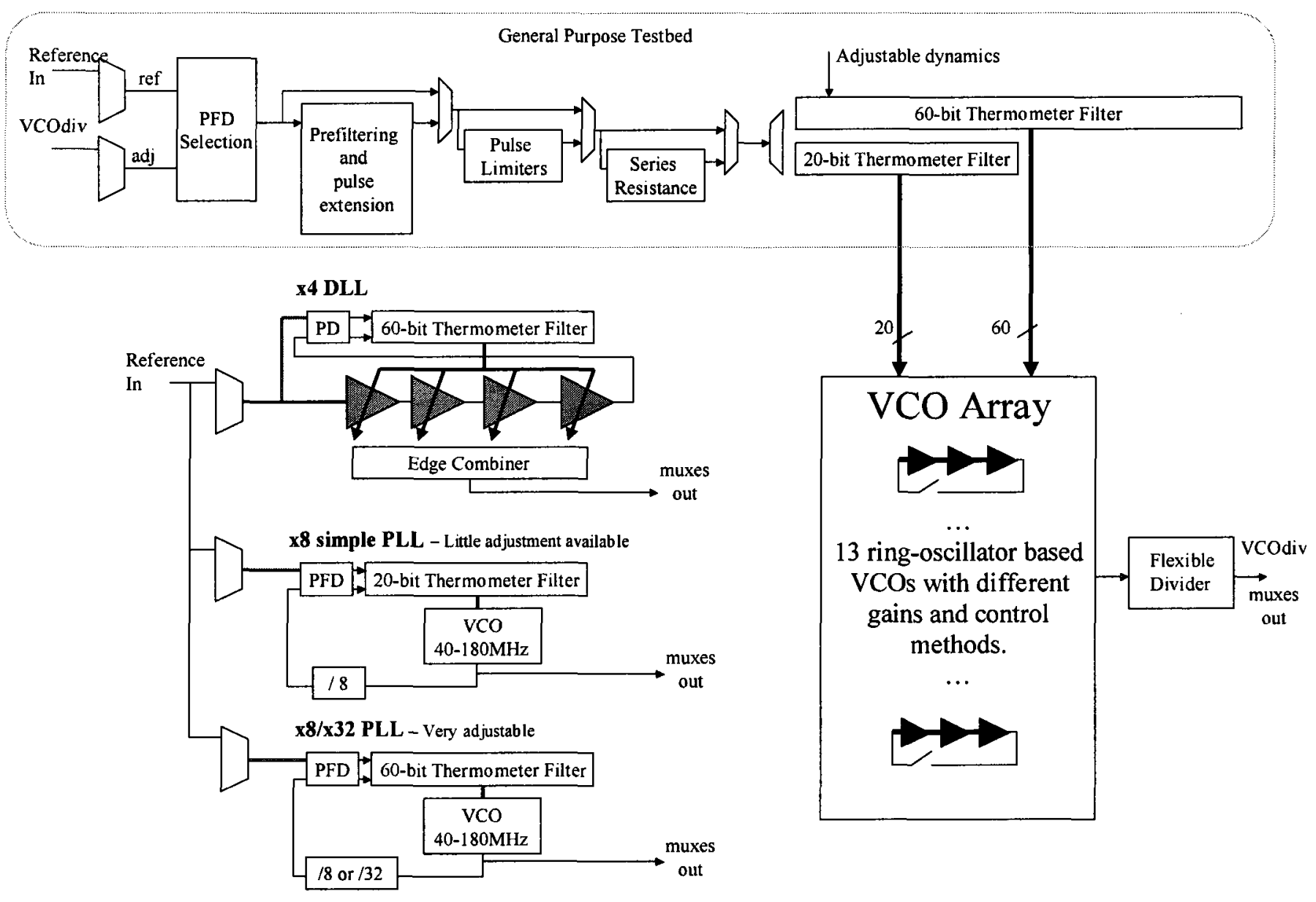

Figure 5.2: Block Diagram of the 2nd Prototype

The control for the general purpose testbed is more fully described in Figure 5.4. This circuit permitted, for example, different PFDs to be selected, coupled through different configurations of prefilters/bias circuitry, into either a 20,40 or 60 


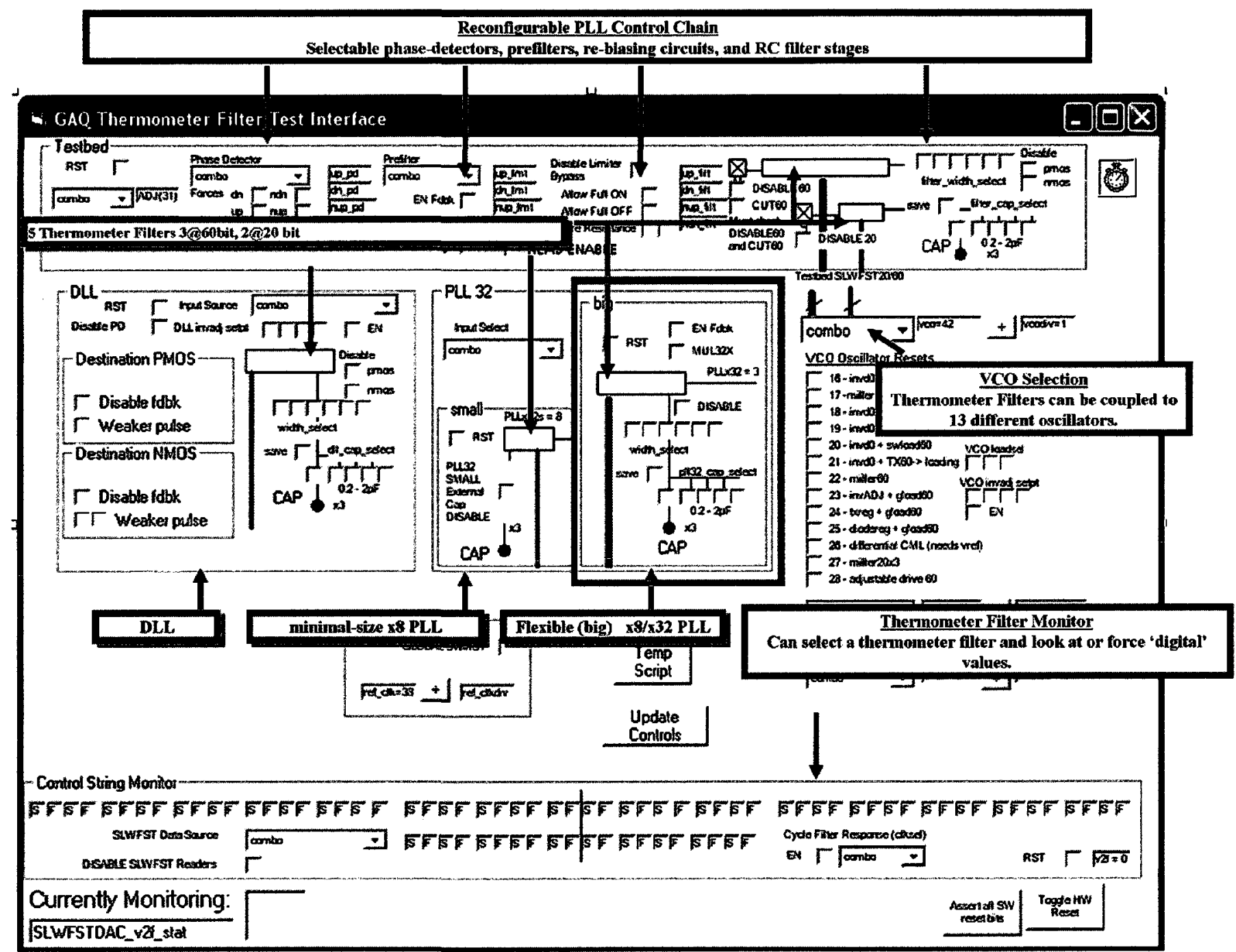

Figure 5.3: Control Software 
stage cascaded charge-pump, and then to a variety of different VCOs. Unfortunately, a bug during clock tree synthesis resulted in a poor clocking structure and a hold time violation within the serial control interface. This left many sections of the chip, including the general purpose testbed, with either no control, or bits that would be haphazardly populated during serial accesses.

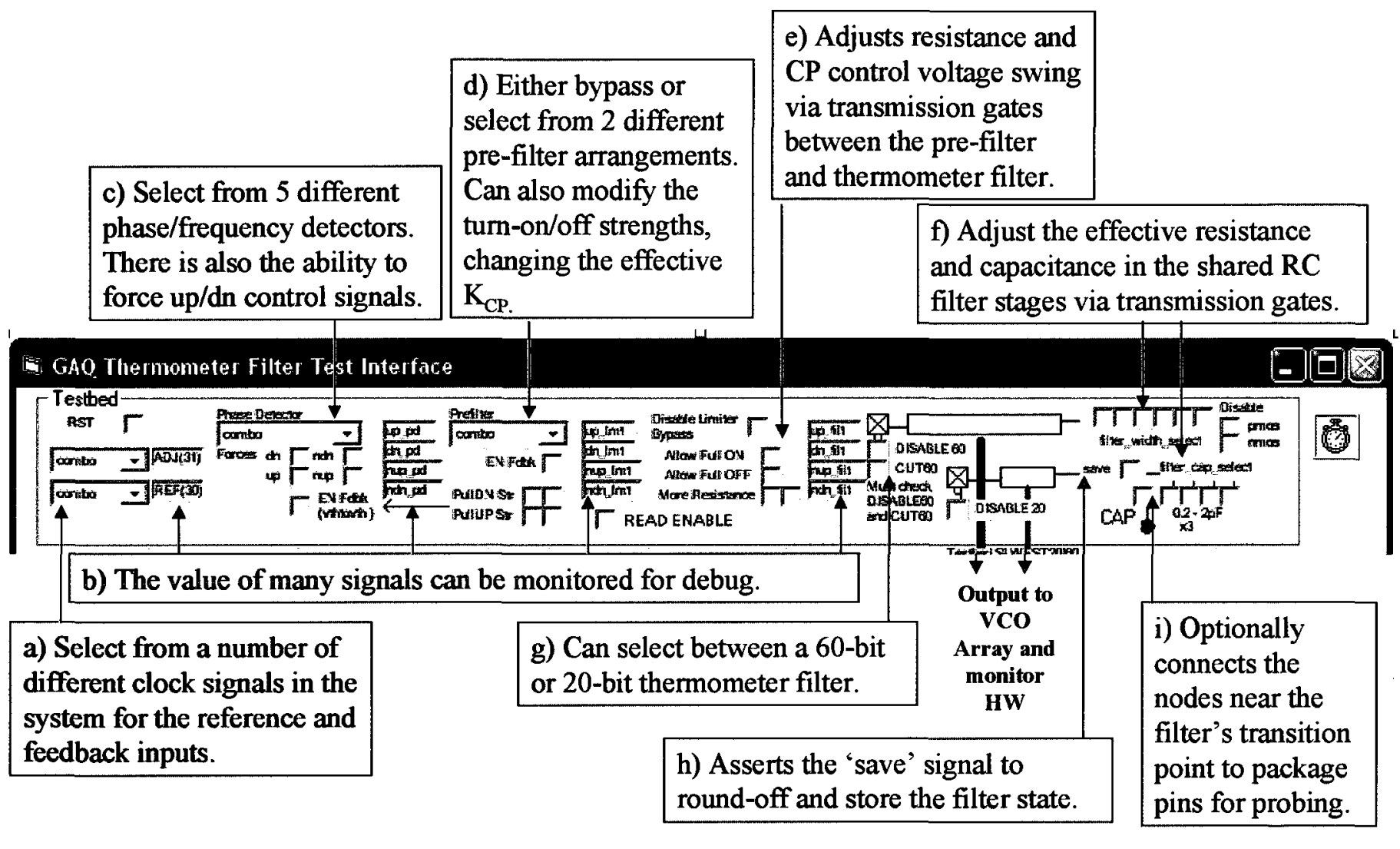

Figure 5.4: Testbed Control

While the loss of this testbed was unfortunate, another important circuit on the die, the Flexible (Big) $x 8 / x 32$ PLL shown in Figures 5.2 and 5.3, was still fully controllable.

\subsection{0-Stage Cascaded-Pump x8/x32 PLL}

A simplified schematic for the example PLL is shown in Figure 5.5. As usual, it contains a phase-frequency detector, a controlled oscillator and a controllable frequency 
divider. It also uses a prefilter circuit and 60-bit cascaded charge-pump and filter, which are the subject of this section.

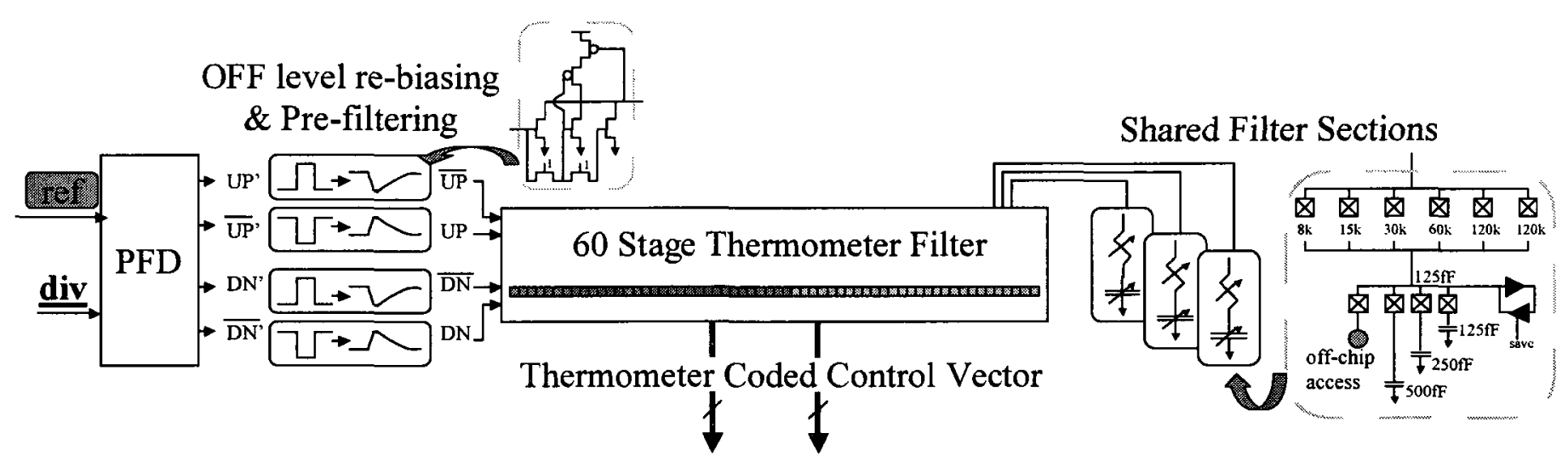

Ring Oscillator: 30 active high +30 active low control bits Divide by $8 / 32$

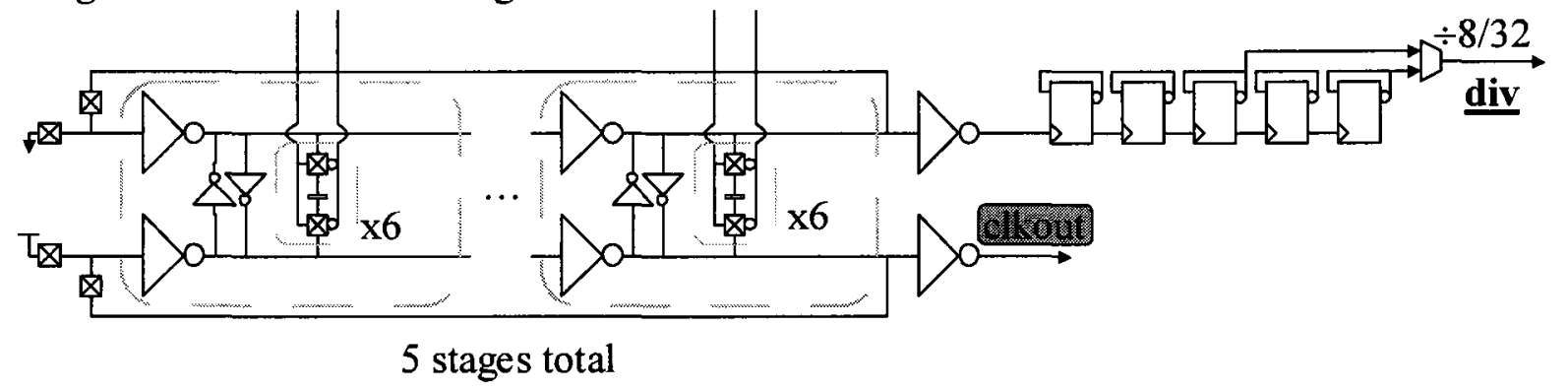

Figure 5.5: PLL Implementation

\subsubsection{PFD and Prefiltering}

A standard 2 flip-flop phase-frequency detector [11] is followed by the prefilters which perform pulse-width extension and voltage re-biasing as in Section 4.10. The prefilter has a number of advantages: it increases charge-pump gain without harmful current spikes and feedthrough spurs, it increases the charge-pump sensitivity to very small phase errors, it reduces the voltage swing and thus power consumption on the control lines, and it creates a higher order pole in the transfer function to smooth the UP/DN control pulses, reducing coupling and sampling problems (spurs). The disadvantage, however, is that the response (or gain) to very small phase errors, while dramatic, can vary significantly with process conditions. This can introduce a dead-zone, which is visible as a small systematic jitter near the 0-phase mark as the phase gets kicked 
from high to low gain regions. This is visible in simulations included in the appendix. Nevertheless, when the dead-zone avoidance pulses from the PFD are wide enough to more-fully activate the pumps this variations is not significant.

The simulated pump gain, under influence of the PFD and prefilter is shown in Figure 5.6. Simulations show the mean pump current as $I_{C P} \approx 15 u A\left(K_{C P}=\right.$ $\left.I_{C P} / 2 \pi\right)$. Zooming in around the 0-phase mark, the effect of using the prefilter with a small dead-zone width $(\Delta)$ is apparent as the charge-pump current rises up from $15 \mathrm{uA}$ to $120 \mathrm{uA}$ for small phase errors. The asymmetry of this extra gain, however, can be problematic as it may result in a small steady state deterministic jitter depending on the process conditions. This is shown in the simulation results of Figure B.14 contained in the appendix.
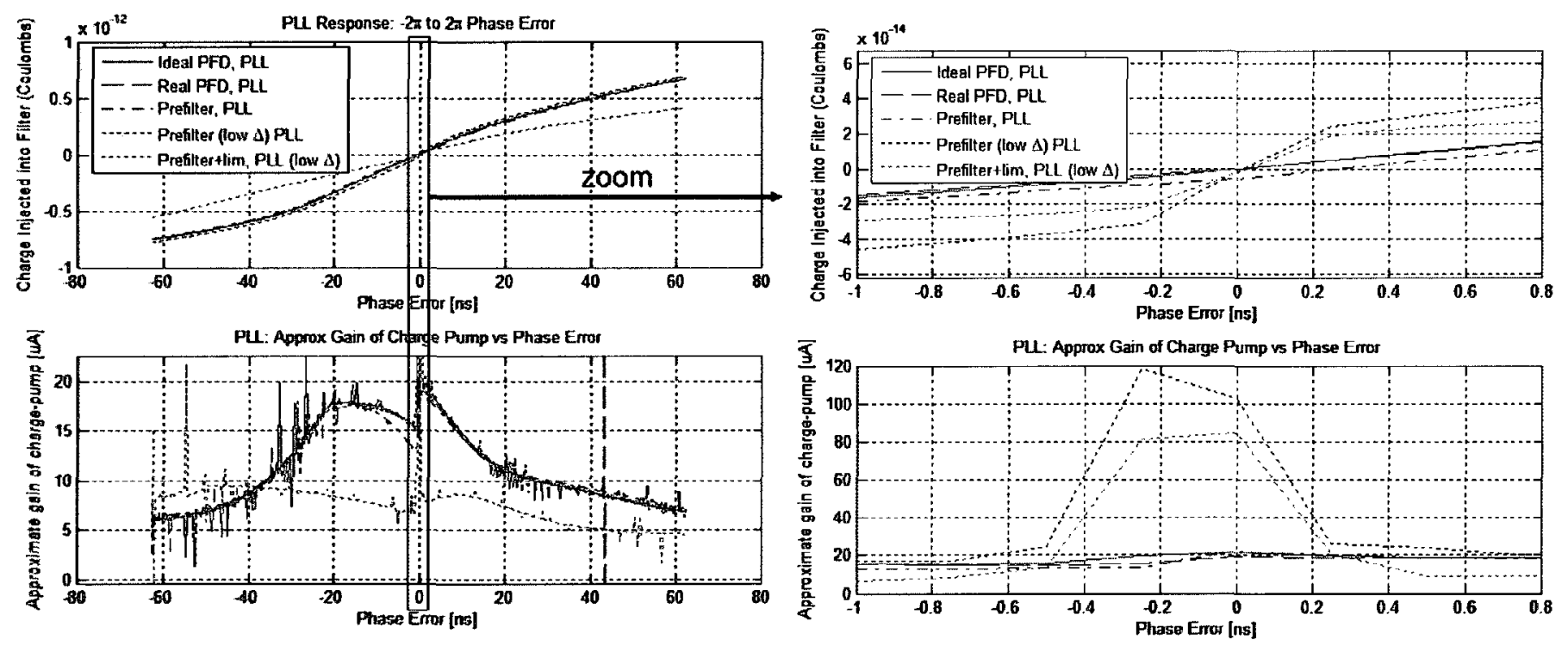

Figure 5.6: Simulated Charge-Pump Gain With/Without prefiltering

\subsubsection{Controlled Oscillator}

The ring oscillator, shown in Figure 5.5, consists of 5 stages with standard rail-torail CMOS inverters. It uses a pseudo-differential technique, where two delay-lines of opposite polarity are coupled together with back-to-back inverters at each stage, as suggested by Kwasniewski [29]. This structure has two benefits: If one of the 
lines, for some transient reason, advances too quickly or slowly, the other line will work to resist that change and reduce jitter. The structure also provides some supply rejection. The back-to-back inverters between the lines form a change resistant latch. Supply or ground bounce changes the speed in the drive inverters, but is countered by the similar changing strength of the latch. The schematic for the VCO stage is available in the appendix, Figure B.6.

To control the oscillation frequency, capacitance is exposed between the two pseudo-differential rings. With opposing voltage swings across the capacitor, Miller multiplication increases the effective capacitance. Changing the voltage level on the switch transistors gives the capacitance more or less exposure to the line, and so the mixed-signal input has a modulating (though not necessarily linear) effect on delay. There are a total of 30 Miller capacitors, 6 per stage, that can be exposed between the two rings. Due to the large number of control bits, even when the switch transistors are off there is still a large parasitic load on each net of the oscillator. The fabricated VCO had a measured range between $43.2 \mathrm{MHz}$ and $172 \mathrm{MHz}$. Though low for many academic chips, it should be recognized that the vast majority of digital ASICs and FPGAs in $0.18 \mu \mathrm{m}$ are clocked within these frequencies. It is also straightforward to extend or modify this range through transistor and capacitance sizing.

\subsubsection{Top Level Specifications and Die-Photo}

A number of important specifications are summarized in Figure 5.8. In the diephoto of Figure 5.7, the relevant region is exploded and the actual PLL components themselves are highlighted. The surrounding area is conventional digital logic, and in clock management roles would include the leaf flip-flops clocked by this PLL instance.

With adjustable loop dynamics, extra capacitance and resistance can be switched in or out. The area figures are given for a minimal working configuration, and for one including all of the extra RC.

\subsubsection{Measured Transient Response}

Figured 5.9 shows the measured transient response of the PLL, configured as an $8 \mathrm{x}$ multiplier, for an input frequency step from 14 to $16 \mathrm{MHz}$. The plot shows the voltage levels on the three shared filter sections (see the 'off-chip access' label on 


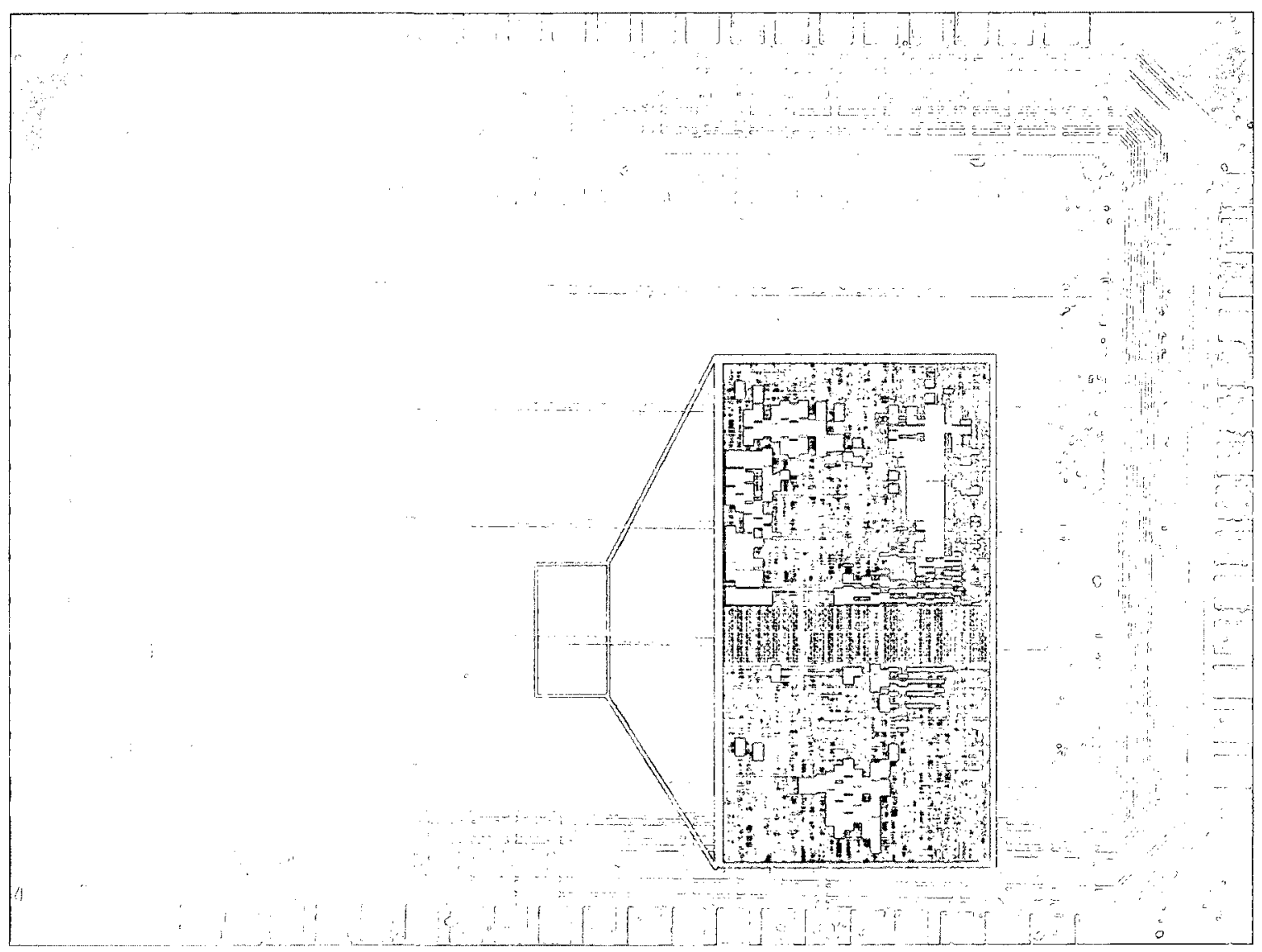

Figure 5.7: Die Photo: Focus on region near PLL. Only the highlighted components are parts of the PLL in question, including the filter capacitance which is implemented as standard-cell MOSCAPs. The 60 element cascaded charge-pump is formed in three pieces (20 elements each), and is recognizable in the top-right section as the three large vertical slices. The remainder of the die contains many other PLLs and DLLs, with a block-diagram shown in Figure 5.2. 


\begin{tabular}{|c|c|c|c|c|c|c|c|}
\hline & \multirow[b]{2}{*}{ Enits } & \multirow[b]{2}{*}{ GOAL } & \multicolumn{3}{|c|}{ Simulated } & \multirow[b]{2}{*}{ Measured } & \multirow[b]{2}{*}{ NOTES } \\
\hline & & & Min & Typ & $\max$ & & \\
\hline \multicolumn{8}{|l|}{ AREA Consumption } \\
\hline Pfo and Prefiters & gates & & & 26 & & & \multirow{8}{*}{$\begin{array}{l}\text { 12.2um }{ }^{2} \text { gate in TSMC } 0.48 \text { um CMOS, Min/Max area } \\
\text { apply because loop-filter passives can be switched inhout } \\
\text { and when switched out are not considered pant of the } \\
\text { circuit size. }\end{array}$} \\
\hline ved & gates & & & 54 & & & \\
\hline Thermameter $\mathrm{H}$ W & gates & & & 156 & & & \\
\hline Dyiger & gates & & & 27 & & & \\
\hline Adjustable R & gates & & 3 & 3 & 81 & & \\
\hline Adiustable c & gates & & 21 & 304 & 304 & & \\
\hline TOTAL Area & gates & $<1000$ & 287 & 570 & 648 & & \\
\hline TOTAL Area & $4 \operatorname{mi}^{2}$ & & 3501 & 6954 & 7906 & & \\
\hline \multicolumn{8}{|l|}{ Power, VCO Range, BW } \\
\hline Supply & $v$ & & 1.6 & 165 & 1.8 & & \\
\hline VCO Min Freo & $\mathrm{HHz}$ & & 41.58 & 55.8 & 69.1 & 43.2 & \multirow{3}{*}{$\begin{array}{l}\text { Fixed P\&R parasitics not accurately annotated. } \\
\text { NFETRPET imbalance can cause latch based VCO freq } \\
\text { to change dramatically }\end{array}$} \\
\hline VCO Max Freq & 1942 & & 237 & 332.2 & 4366 & 172 & \\
\hline Kroo & $M H Z N$ & & 197 & 28 & 3.71 & 13 & \\
\hline & & & & & & & \\
\hline VoO Current & w & & $156-175$ & $218-254$ & $307-333$ & & \\
\hline Total Current Cons & UA & $<500$ & & & & 121 & Rparasitcs in vCo contribute to lower freg and current \\
\hline & & & & & & & \\
\hline 3dB Closed Loop BW & & & & $200 \mathrm{kHz}$ & $200 \mathrm{kHz}$ & $200 \mathrm{kHz}$ & \\
\hline \multicolumn{8}{|l|}{ Phase Noised Jitter } \\
\hline $128 \mathrm{HH} \mathrm{YVCO}$ & & & NOTE 1 & & NOTE 2 & & \\
\hline $300 \mathrm{~Hz}$ & $\mathrm{dec} / \mathrm{Hz}$ & $<-70$ & -85 & & -85 & & \multirow{13}{*}{ 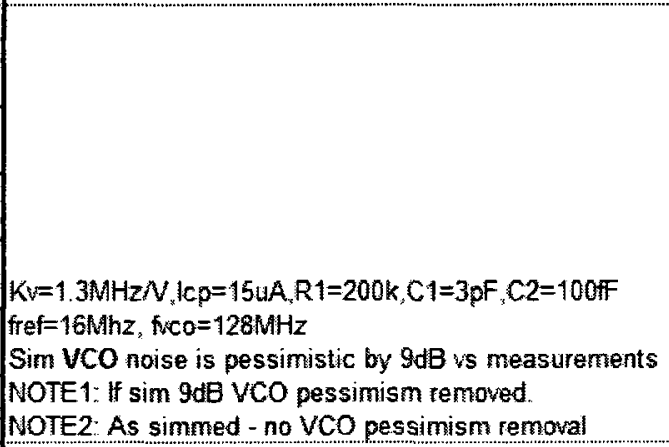 } \\
\hline $1 \mathrm{kHz}$ & $\mathrm{dBCHz}$ & $<-70$ & -90 & & -90 & & \\
\hline $8 \mathrm{kHz}$ & $\mathrm{dBCH}$ & $<-70$ & -100 & & -97 & -100 & \\
\hline $10 \mathrm{kHz}$ & $\mathrm{SBCHE}$ & $<-70$ & -100 & & -96 & -100 & \\
\hline $20 \mathrm{kHz}$ & $\mathrm{OBOHz}$ & $<-70$ & -100 & & -95 & -100 & \\
\hline $40 \mathrm{kHz}$ & $\mathrm{dBcHz}$ & -70 & .99 & & -92 & -98 & \\
\hline $80 \mathrm{kHz}$ & $\mathrm{OBCHZ}$ & $=-70$ & -95 & & -86 & -95 & \\
\hline $100 \mathrm{kHz}$ & $\mathrm{dBCHz}$ & $\begin{array}{l}<-70 \\
-30\end{array}$ & -93 & & -84 & .94 & \\
\hline $200 \mathrm{kHz}$ & $20 \mathrm{cHz} z$ & $<-70$ & -93 & & -84 & -94 & \\
\hline $300 \mathrm{kHz}$ & $\mathrm{dEciHz}$ & -70 & -101 & & -92 & -102 & \\
\hline $500 \mathrm{kHz}$ & $\mathrm{ABCHHZ}$ & $4-70$ & -108 & & -99 & -108 & \\
\hline $1 M$ & $\mathrm{ABCHZ}$ & $<-70$ & -116 & & -107 & -116 & \\
\hline $10 \mathrm{M}$ & $\mathrm{dBCHz}$ & $<-70$ & -142 & & -133 & & \\
\hline Banerjee FOM @ $10 \mathrm{kHz}$ & $6 \mathrm{BC}$ & & -183 & & -183 & & $P N-20 \log (N)-10 \log (f \mathrm{fef})$ \\
\hline Integrated Noise $100 \mathrm{~Hz}-1 \mathrm{MHz}$ & $\mathrm{dBc}$ & & -382 & & -29.4 & -38 & \\
\hline Integrated Noise $8 \mathrm{kHz}-1 \mathrm{MHz}$ & $d B c$ & & -38.3 & & -29.5 & -36 & \\
\hline Integrated Noise 8idzz-10MHz & $\mathrm{dBc}$ & & -383 & & -29.5 & -38 & \\
\hline RMS Integrated Jitter & os & & & 95 & 261 & 95 & Calculated via integrated phase noise $100 \mathrm{~Hz}-10 \mathrm{MHz}$ \\
\hline DC Phase offset & $p s$ & $<50$ & 0.4 & 6 & 12 & & \\
\hline Systematic Jitter (rms) & ps & & 0 & & 5.4 & & Due to dead-zone variation wi process conditions \\
\hline period jitter (peak) & ps & $<100$ & & & & 37.8 & \\
\hline perod jitter (rms) & ps & & & & & 5.6 & Observed over a span of 3000 cycles \\
\hline \multicolumn{8}{|l|}{ OP Current } \\
\hline- Ins to ins & UA & & 13 & 15 & 18 & & \multirow{2}{*}{$\begin{array}{l}\text { Variation across phase offset under typical procitemp, } \\
\text { wide UPIDN puses }\end{array}$} \\
\hline $40 \mathrm{~ns}$ to $40 \mathrm{~ns}$ & UA & & 8 & 15 & 19 & & \\
\hline UPSON mismatch & UA & & 0 & 1 & 2 & & Across $-100 p s$ to $+100 p s$ \\
\hline & & & & & & & \\
\hline \multicolumn{8}{|l|}{ Loop Filter Components } \\
\hline R1 at max setting & $\mathrm{kg}$ & & 24 & 200 & 250 & & \multirow{6}{*}{$\begin{array}{l}\text { Section includes variation across bias point, not process } \\
\text { Low value of } 24 \mathrm{k} \Omega \text { leads to only } 4.5 \text { deg phase margin and } \\
\text { instability at low voltage lock points. } \\
\mathrm{R} 1=200 \mathrm{k} \Omega \mathrm{C} 1=3 \mathrm{pF}, \mathrm{lcg}=15 \mathrm{uA}, \mathrm{KV}=1.3 \mathrm{kHzN}\end{array}$} \\
\hline C1 at min setting & $\mathrm{pF}$ & & 0.2 & & 0.3 & & \\
\hline $\mathrm{Cl}$ at mid setting & of & & 3.0 & & 42 & & \\
\hline C1 at max setting & DF & & 6.0 & & 8.5 & & \\
\hline & & & & & & & \\
\hline Phase Margin & deg & & 4.5 & 35.9 & 50.5 & & \\
\hline
\end{tabular}

Figure 5.8: Specifications: Simulated vs Measured Performance Summary 


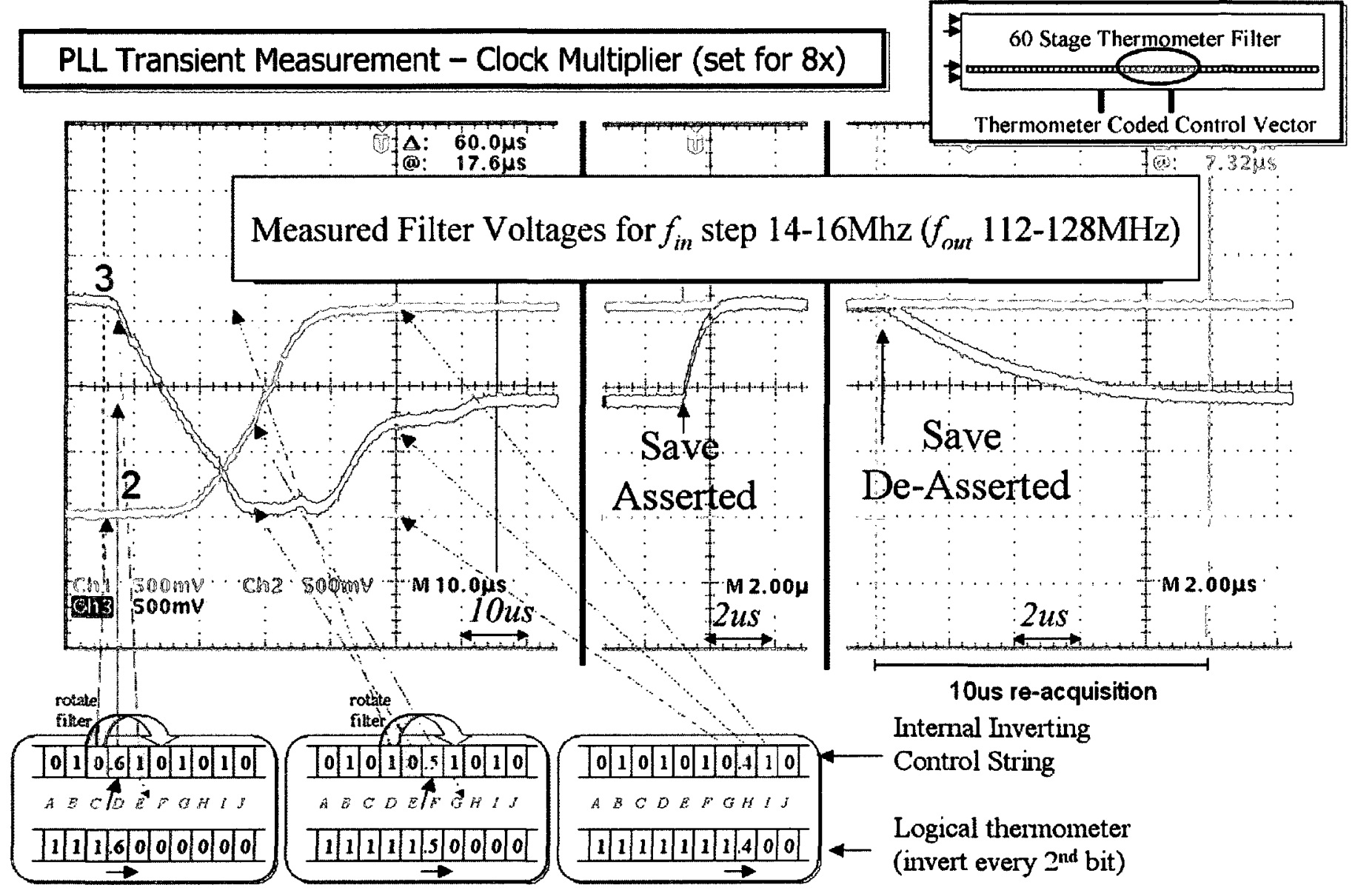

Figure 5.9: Measured Transient Response of Shared Filter Sections

Figure 5.5), and provides a window to the 3 nodes at the codes transition point. In Figure 5.9, control nodes $\mathrm{D}, \mathrm{G}$ and $\mathrm{J}$ are rotated among one capacitor, nodes $\mathrm{C}, \mathrm{F}$ and I share another capacitor, and the third capacitor is switched between nodes $\mathrm{E}$ and H. During lock, as the thermometer code progresses node-by-node, each filter is internally disconnected from a recently stable control and rotated to a node 3 positions away, in preparation to act again on behalf of another node. The capacitance rotation was engineered to ensure that charged capacitances are only switched onto logic ' 1 ' nodes, and discharged caps only connect to nodes which are at logic ' 0 '. This prevents spurious transitions which would occur if connecting charged capacitances to discharged control nets and vise-versa. 


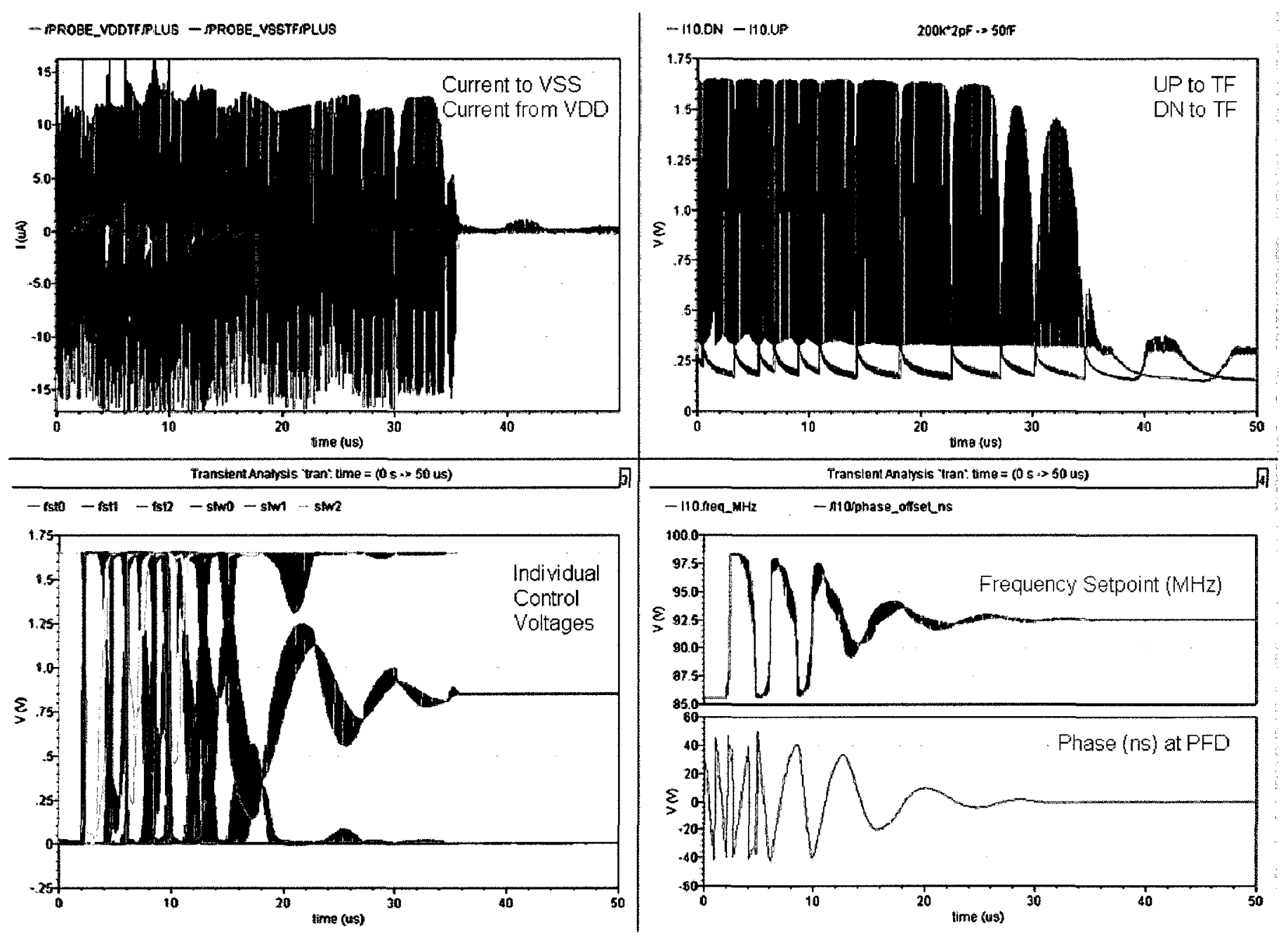

Figure 5.10: Simulated Transient Response of Locking PLL: a) Total supply current to/from Cascaded Charge-Pump. b) Conditioned/rebiased UP/DN control pulses from PFD to CCP. c) Individual VCO control node voltages. d) Frequency setpoint (Sum of individual control voltages ${ }^{*} K_{V C O}$ ), and phase error that hits the phase detector (in ns). 
The capacitance rotation continues until eventually node $\mathrm{H}$ settles into a position where the PLL locks. In the second panel of Figure 5.9, the state-saving latches (Figure 4.12 and Figure 5.5) are enabled. This locks node I at VDD, node J at VSS (where they happen to be already), and snaps node $\mathrm{H}$ to the closest digital rail, rounding the analog lock voltage to VDD and holding it there indefinitely. When the latches are disabled the system recovers quickly from this position. Unfortunately, when probing the control voltages the pad and scope probes add to the effective filter capacitance, reducing the dominant pole from its adjustable value (between $138 \mathrm{kHz}$ and $10 \mathrm{MHz}$ ) to below $10 \mathrm{kHz}$. The transient then, while generally informative, is not indicative of the actual lock and re-acquisition times. As a relative measure, however, it took $\approx 60 u S$ for the relatively small step response to settle, and only $\approx 9 u S$ to recover from the nearest digital lock-state.

A full transistor level simulation of the PLL locking, without the parasitic loading of a probe, is shown in the transient of Figure 5.10. Note that in the simulation results, the actual control voltages are shown, whereas the measured response is limited to observation of the internal loop filter node between $R_{1}$ and $C_{1}$, which is a low-pass version of the actual VCO control.

\section{Stability}

There was a problem using transmission gates to implement the resistor in the loopfilter. The resistance of the TX gate varies significantly, from $20 \mathrm{kOhm}$ to $200 \mathrm{kOhm}$ depending on bias voltage. Simulations of this effect are shown in Figure 5.11. This led to instability when low lock-voltages were called for. The effect was reproduced in simulation. Future implementations should avoid this approach and use resistors instead. A slightly more detailed look at the circuit and simulation results is available in the appendix, in Figure B.9.

\subsubsection{Jitter, Phase-Noise and Power Consumption}

Using the PLL as an $8 \mathrm{x}$ clock multiplier, the measured period jitter and a wideband plot of the phase-noise is shown in Figure 5.12. The jitter histogram, in particular, 

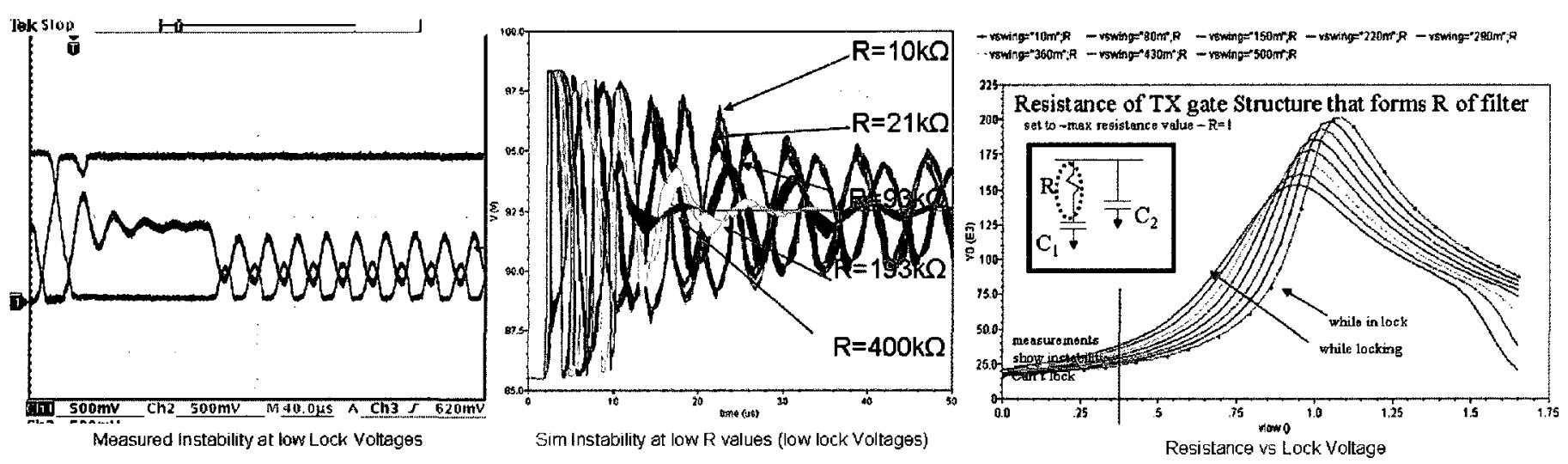

Figure 5.11: Instability: Observed Instability at low lock voltages due to low resistance of TX gate at low bias voltages

contrasts the $16 \mathrm{MHz}$ reference input ${ }^{1}$ with the sanitized $128 \mathrm{MHz}$ PLL output. Even with excessive input jitter $\left(21 p s_{r m s}, 149 p s_{p p}\right)$, the output jitter is only 6.6ps $s_{r m s}$ (or $\left.0.2_{r m s}^{o}\right), 46 p s_{p p}$ which is more than suitable for digital clocking.

The simulated and measured phase-noise, on a logarithmic scale, is presented in Figure 5.13. While the in-band contributions from the charge-pump and loop dynamics match quite well, the simulated VCO noise was pessimistic by $9 \mathrm{~dB}$, and the discrepancy at large offsets is obvious in 5.13a. If an empirical $9 \mathrm{~dB}$ improvement is applied to the simulated VCO characteristic (5.13b), the full closed loop synthesizer simulated and measured data align with almost perfect correlation.

\section{VCO Phase-Noise Measurement vs Simulation}

Large signal PSS spectre simulations of the schematic VCO are pessimistic by $9 \mathrm{~dB}$ compared to measurements. The in-band noise, caused by the charge-pump and remainder of the synthesizer, however, is accurately predicted. The cause of the $9 \mathrm{~dB}$ simulator pessimism on the $\mathrm{VCO}$ is unknown, but there are a number of potential sources of error:

- Simulations are for schematic with estimated parasitics - extracted would not converge

\footnotetext{
${ }^{1} \mathrm{~A}$ sinusoidal reference passes into the IC through a limiting CMOS driver which introduces jitter. It then feeds the PLL input, and can also be switched through the same output path as the PLL to monitor its characteristics.
} 

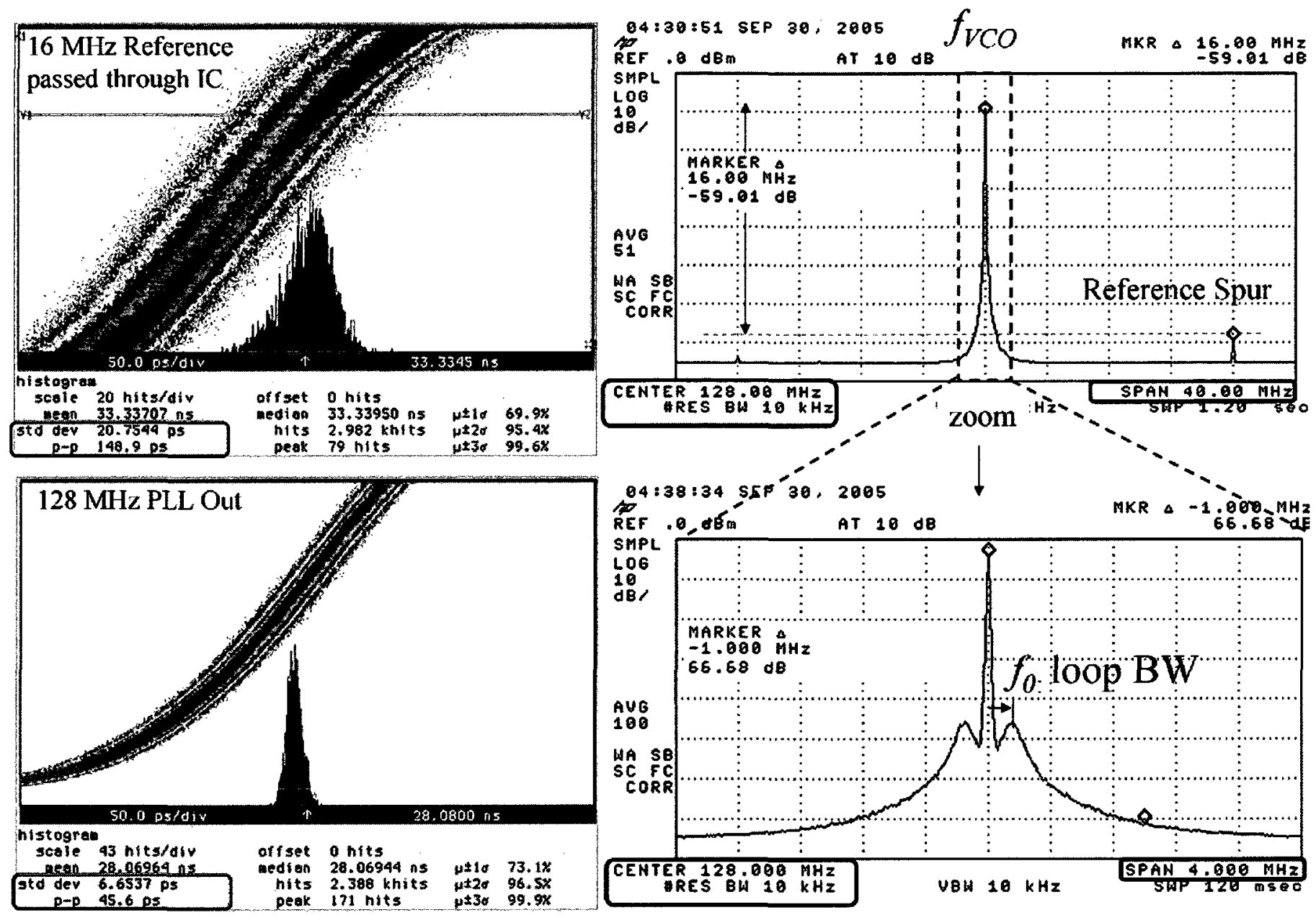

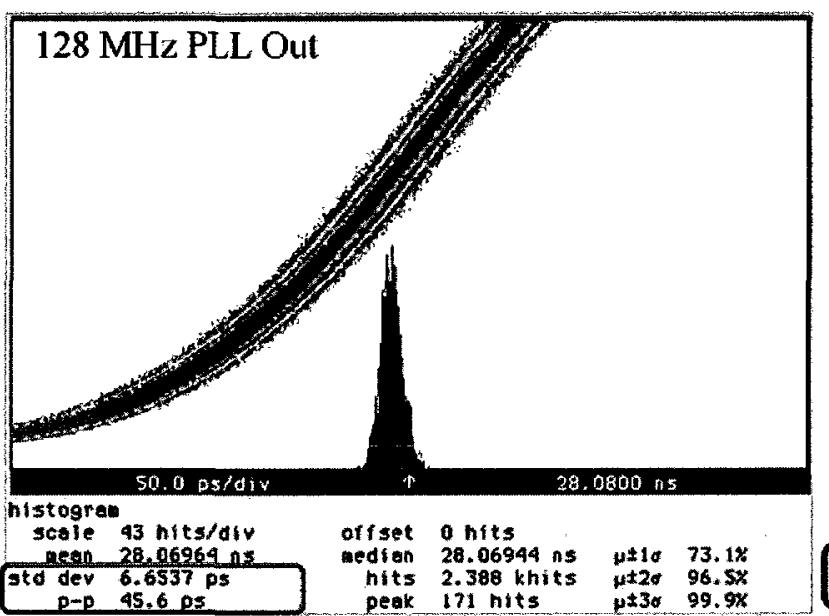

a) Reference and Output Cycle-to-Cycle Jitter Histograms

b) Wideband Frequency Spectrum

Figure 5.12: Measured Period Jitter and Wideband Phase-noise

- Poor manual parasitic back-annotation for RC balance

- PFET/NFET process imbalance effects latch-based VCO

- Model Accuracy

\subsection{Conclusion}

The cascaded charge-pump has been implemented in a $\mathrm{x} 8 / \mathrm{x} 32$ PLL with a ringoscillator based VCO and in this chapter, its simulated and measured performance 

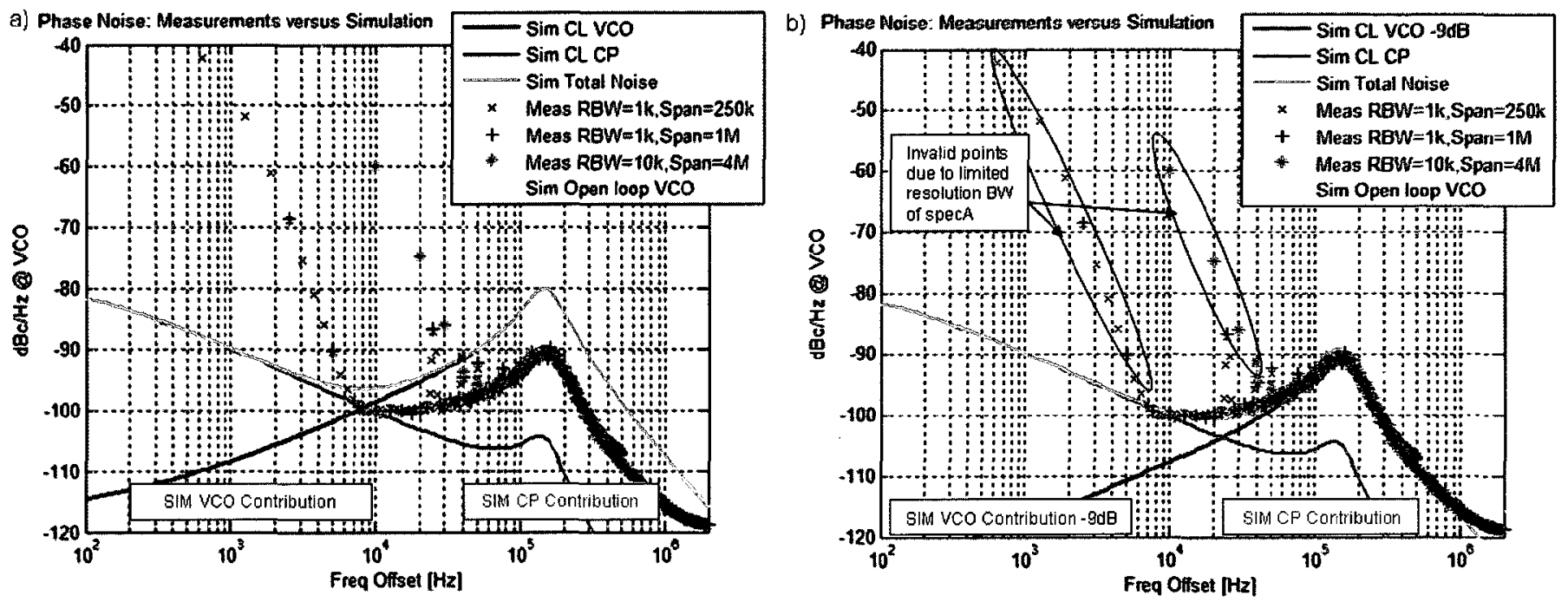

Figure 5.13: Phase-Noise Simulation versus Measurement

a) As simulated - Simulated VCO noise was pessimistic by $9 \mathrm{~dB}$ as evidenced by the out of-band offset between measured data and simulation results

b) With a $-9 \mathrm{~dB}$ correction to simulated VCO noise, total measured and simulated responses match to within $1 \mathrm{~dB}$ across the entire band

has been presented. The cascaded charge-pump (the subject of this thesis) behaves as predicted, as evidenced by the transient plot of Figure 5.9 and the in-band phase-noise shown in Figure 5.13. The VCO, however, ran at a lower frequency than simulated, and had $9 \mathrm{~dB}$ better noise performance than expected. The frequency difference is easily explained by the use of minimally sized transistors coupled with poor parasitic estimates, however the phase-noise improvement is more difficult to explain. The entire PLL, including the VCO, consumed only $I_{\text {total }}=121 u \mathrm{~A}$ and $7906 \mathrm{um}^{2}$, while achieving 46ps peak-to-peak, period jitter. The measured range of the VCO is from $43 \mathrm{MHz}$ to $172 \mathrm{MHz}$, while maintaining a $K_{V C O}<2 M H z / V$ and avoiding bandswitching problems that plague dual-loop architectures. 


\section{Chapter 6}

\section{Conclusions}

\subsection{Summary}

The focus of this thesis has been the analysis and design of phase-locked loops and delay-locked loops, with a concentration on efficient synthesizers for use in clockcontrol and high-speed serial communications. The analysis weighs different architectural choices, and proposes a new mixed-signal structure to drastically reduce the filtering requirements and size of these circuits. The size improvements come about by breaking what is normally a single analog VCO control voltage into a large number $(N)$ of independently controlled segments. The analysis, supported by a custom PLL simulator and measurements, shows that since each segment has a small gain relative to the total, the filter size can by reduced by $\approx N$ times while maintaining the same loop dynamics. A unique cascaded-charge pump has been designed to control this type of VCO and was implemented using an analog standard-cell methodology, where the analog design is automatically placed \& routed using commercial EDA tools designed for digital circuit implementation.

The cascaded charge-pump is described at a relatively high level of abstraction in Chapter 3. The analysis shows that the effective reductions in VCO gain can be traded for either reduced capacitance and smaller circuit size, or for higher chargepump gain and better noise performance. With this second approach, the improved noise performance extends the optimal loop bandwidth of the overall solution, also allowing a reduction in capacitance but accompanied by a lower noise solution. The chapter describes how the core of the circuit is formed by a somewhat odd connection of tri-state digital gates. An analysis is also presented on the complications of transferring VCO control from one segment to the next, and the potential implications 
of any non-linearity of this transition. A PLL simulator was written to characterize a number of these effects (and others), and runs approximately 20,000x faster than transistor level simulations and 300x faster than other behavioural simulators.

More detailed circuit level design and implementation issues are covered in Chapter 4. Here, further simplifications of the cascaded charge-pump are presented, allowing the fundamental charge-pump cell to be constructed with as few as 4 transistors each. Further analysis discusses how to perform analog filter multiplexing, and the implications of charge-pump saturation, mismatch and leakage. Also addressed is a novel approach to save the nearest 'digital' state of the system using only 3 small latches, despite the number of VCO control segments.

The appendices contain a number of useful sections. Appendix A outlines how the PLLs and DLLs developed here can be used to solve clocking issues in digital systems. Appendix $\mathrm{C}$ provides a guideline to design an optimal synthesizer to meet a specified phase-noise mask, and Appendix D contains a unique treatment of jitter and its relationship to phase-noise.

Out of approximately 100 different PLLs and DLLs implemented using a semiautomatic synthesis engine, one particular PLL design is highlighted with both simulation and measurement results. The innovative cascaded charge-pump control structure has been used to create the smallest and lowest power PLL ever reported, by a very wide margin. A literature survey focusing on synthesizers with similar goals is given in Table 6.1.

The goal of the thesis was to invent a synthesizer architecture with drastically reduced size and power consumption, while maintaining an acceptable level of spectral purity. The quantitative measure of this success, is the product of areaøpower jitter. As noted in Table 6.1, this FOM comes in at $0.07\left(0.008 \mathrm{~mm}^{2} \bullet 0.2 \mathrm{~mW} \bullet 46 \mathrm{ps}\right)$ for this work, versus 32 from the closest other competition [30]. This is an advantage of $450 \mathrm{x}$, or 2.5 orders of magnitude. Furthermore, if one were to pick-and-chose the very best area/power/jitter numbers from the available solutions (which is of course unrealistic), this fictitious synthesizer has a figure of merit of $0.07 \mathrm{~mm}^{2} \bullet 2.10 \mathrm{~mW} \bullet 19 \mathrm{ps}=2.8$, which is still $40 \mathrm{x}$ poorer than this work. 


\begin{tabular}{|c|c|c|c|c|c|c|c|}
\hline & Type & Year & Speed & Area & Power & T Jitter & FOM \\
\hline \hline This & Mixed & 2006 & 60 to & $0.008 \mathrm{~mm}^{2}$ & $0.19 \mathrm{~mW}$ & $6.7 p s_{r m s}$ & 0.07 \\
Work & & $0.18 \mu \mathrm{m}$ & $172 \mathrm{MHz}$ & 650 gates & $@ 128 \mathrm{MHz}$ & $45.6 p s_{p p}$ & \\
\hline$[7]$ & Analog & 2000 & 8.5 to & $0.09 \mathrm{~mm}^{2}$ & $25 \mathrm{~mW}$ & $50 p s_{p p}$ & 112 \\
Ahn & & $0.25 \mu \mathrm{m}$ & $660 \mathrm{MHz}$ & & $@ 144 \mathrm{MHz}$ & & \\
\hline$[6]$ & Analog & 1996 & $0.002 \mathrm{to}$ & $1.91 \mathrm{~mm}^{2}$ & $9.2 \mathrm{~mW}$ & $144 p s_{p p}$ & 2530 \\
Maneatis & & $0.5 \mu \mathrm{m}$ & $550 \mathrm{MHz}$ & & $@ 500 \mathrm{MHz}$ & & \\
\hline$[15]$ & ADPLL & 2003 & 30 to & $0.31 \mathrm{~mm}^{2}$ & $3.12 \mathrm{~mW}$ & $60 p s_{r m s}$ & 125 \\
Fahim & & $0.25 \mu m$ & $160 \mathrm{MHz}$ & & $@ 144 \mathrm{MHz}$ & $130 p s_{p p}$ & \\
\hline$[24]$ & ADPLL & 2003 & $45 \mathrm{to}$ & $0.71 \mathrm{~mm}^{2}$ & $100 \mathrm{~mW}$ & $22 p s_{r m s}$ & 4970 \\
Chung & & $0.35 \mu m$ & $510 \mathrm{MHz}$ & & $@ 500 \mathrm{MHz}$ & $70 p s_{p p}$ & \\
\hline$[22]$ & Analog & 2006 & $100 \mathrm{MHz}$ & $0.09 \mathrm{~mm}^{2}$ & $12 \mathrm{~mW}$ & $7.1 p s_{r m s}$ & 70 \\
Shi & & $0.35 \mu m$ & to $560 \mathrm{MHz}$ & & $@ 350 \mathrm{MHz}$ & $65 p s_{p p}$ & \\
\hline$[30]$ & Analog & 2008 & $2500 \mathrm{MHz}$ & $0.08 \mathrm{~mm}^{2}$ & $21 \mathrm{~mW}$ & $19 p s_{p p}$ & 32 \\
Cheng & & $0.13 \mu \mathrm{m}$ & & & $@ 2500 \mathrm{MHz}$ & & \\
\hline$[2]$ & ADPLL & 2003 & $90 \mathrm{to}$ & $0.07 \mathrm{~mm}^{2}$ & $2.1 \mathrm{~mW}$ & $>300 p s_{p p}$ & 44 \\
Olsson & & $0.35 \mu m$ & $230 \mathrm{MHz}$ & & $@ 90 \mathrm{MHz}$ & & \\
\hline \hline
\end{tabular}

Table 6.1: Comparison vs other low-complexity/power PLLs

The cascaded charge-pump invented here has facilitated the creation of a synthesizer with the following highlights:

- Lowest Power PLL ever : 0.2mW vs $2.1 \mathrm{~mW}[2]$

- Smallest PLL ever : $0.008 \mathrm{~mm}^{2}(0.18 \mathrm{um})$ vs $0.07 \mathrm{~mm}^{2}$ (0.35um) [2]

- Comparable period jitter to other solutions (7ps RMS, 46ps pp)

- Competitive phase-noise for the application : Banerjee FOM of $-183 \mathrm{dBc} / \mathrm{Hz}$

- Wide-range ( $>1$ octave, $60 \mathrm{MHz}$ to $172 \mathrm{MHz}$ )

- Automatically synthesized PLL/DLL designs

- Automatically Placed \& Routed with standard-cells

\footnotetext{
${ }^{1}$ The author estimates the equivalent power consumption for this work to run @ $2.5 \mathrm{GHz}$ in $0.13 \mu \mathrm{m}$ would be between $1.2 \mathrm{~mW}-1.8 \mathrm{~mW}$.
} 
- Fully integrated, with no external components

- Does not suffer from quantization jitter

- Save/Recall nearest digital state for quick frequency acquisition

- Adjustable loop dynamics

- Low and predictable $K_{V C O}$

The size advantages are a result of the cascaded charge-pump's effective capacitance multiplication, whereas the power efficiency can be attributed to a PLL control loop which eliminates unnecessary full-swing transitions, a lack of DC bias current, running with a reduced supply voltage $(1.65 \mathrm{~V}$ vs $1.8 \mathrm{~V})$, and the use of a very efficient VCO. Not only do these measurements excel in one dimension, but in all three parameters of interest - the area-power jitter product is over an order of magnitude smaller than any designs uncovered thus far.

\subsection{Contributions}

- A novel architecture for analog integrators which permit integration into a cascade of analog sub-cells, reducing component requirements in terms of area and noise.

- Modification of the aforementioned structure for use as a cascaded charge-pump (CCP) in Phase/Delay locked-loops.

- An analysis of the system level effects/benefits of the CCP. Among the analysis, the following sub-contributions can be identified.

- A method to decouple supply limitations from necessary increases in $K_{V}$ and the associated penalties.

- A corrollory, is a method to reduce filter-component sizes, which are the dominant area cost in PLLs/DLLs.

- Simplifications and analysis of the circuit level implications of the CCP. 
- A method to dynamically identify analog nodes and smoothly multiplex filter components as required.

- Experimental validation of the cascaded integration technique, including the measurements of the smallest and lowest power PLL ever reported.

\subsubsection{Associated research}

In addition to the main thrust of the research, a number of auxiliary contributions are highlighted below.

- An investigation of asynchronous and globally-asynchronous locally-synchronous (GALS) methods, resulting in the successful design/fabrication and test of a GALS Digital Signal Processing IC.

- An accurate (better than $-200 \mathrm{dBc} / \mathrm{Hz}$ noise floor) Closed-loop PLL simulator that model a variety of effects and run 20000x faster than transistor level, 300x faster than other high-level PLL simulators.

- Proven feasibility of analog standard-cell design/integration in synthesizer design.

- Generic design procedure for meeting phase-noise targets with an efficient (lowpower, low-area) design.

- An intuitive and original treatment of the link between phase-noise, integrated jitter and period jitter.

- A simulation method to characterize the gain and linearity of the charge-pump vs phase-error.

\subsection{Publications}

\subsubsection{Refereed}

- G. Allan, J. Knight, 'A compact 190uW PLL for clock control and distribution in ultra-large scale ICs', ISCAS Conference proceedings, 2006 
- G. Allan, J. Knight, 'Mixed-signal thermometer filtering for low-complexity PLLs/DLLs', ISCAS Conference proceedings, 2006

- G. Allan, J. Knight, N.Filiol, T.Riley, 'Digitally Place and Routed Up-converting Bandpass DAC', CCECE Conference proceedings, 2006

- G. Allan, J. Knight, 'Low-Complexity Digital PLL for Instant Acquisition CDR', ISCAS Conference proceedings, 2004

- 'Novel Architecture For Ultra Low Complexity Mixed-Signal DLL', Analog VLSI Workshop, Oct. 2004, Macau, China

\subsubsection{Patents/Patent Applications}

\section{Examined/Approved}

- 'Mixed-Signal Thermometer Filter, Delay Locked Loop and Phase Locked Loop', Patent Cooperation Treaty (PCT) Application PCT/CA2005/001060, Priority: July 8, 2004, Filed: July 7, 2005, Approved: Jan 9, 2007

\section{Pending}

- 'Mixed-Signal Thermometer Filter, Delay Locked Loop and Phase Locked Loop', United States Patent Application 10/568,279, Pub Date: Jun 28,2007

- 'Method and Apparatus for Mixed-Signal DLL/PLL as Useful in Timing Manipulation', Canadian Patent Application 2,474,111, filed July 8, 2004

- 'Mixed-Signal Thermometer Filter, Delay Locked Loop and Phase Locked Loop', Canadian Patent Application, filed July 7, 2005

\subsubsection{Other}

- G. Allan, J.Knight, 'High-Speed Self Synchronizing Serial Interconnections for Systems on a Chip', Micronet Annual Workshop, Toronto, 2003 
- G. Allan, J.Knight, 'Toward Automatic Generation of Globally Asynchronous, Locally Synchronous Clock Domains in SOCs', Micronet Annual Workshop, Ottawa, 2004

- G. Allan, T.Riley, N. Filiol, J. Knight, 'Digitally Integrated DAC, Mixer and Filter for Multi-Standard Radio Transmitters', CITO Innovations, Toronto, Nov 2004

- G. Allan, J. Knight, 'Design and Engineering Test of a Reconfigurable Radio Platform', MR\&DCAN, Ottawa, 2004

\subsection{Future Work}

There are a number of avenues which can continue to be explored in further work along these lines. In particular, there are a number of things the author recommends be revisited in a future design.

\section{Noise Optimization}

In retrospect, the noise performance of the synthesizer can be improved significantly with only minor degradation in power consumption. In particular, the transistor of the prefilter which is responsible for turning off the control node dominates the noise and can easily be resized to improve noise performance - the author estimates that more than $10 \mathrm{~dB}$ improvement can be achieved with negligible cost.

\section{Loop BW optimization}

Though the dynamics in the prototype were adjustable via switchable capacitance, the extreme fluctuations in the switch resistance of the transmission gates of the loop filter limited the available solutions. The achievable loop-BW for stable operation could not be made wide enough to suppress the VCO contributions for optimal performance.

\section{Regulated current sources}

In this thesis simple rail-to-rail switches were used in the cascaded charge-pump as current sources. In combination with the prefilter structures, this made the actual 
charge-pump gain difficult to predict. A more conventional biasing approach may be used on the control lines that turn these transistors into more predictable sources. 


\section{Appendix A}

\section{PLLs and DLLs in Clock Distribution}

\section{A.1 Thesis Application: Digital Clocking}

In digital circuits, the clock is either fed from an external source, or in other scenarios, is generated internally by a PLL or DLL. In either case, it is a significant challenge to control the distribution of this clock internally.

\section{A.1.1 How Clock Delays lead to Circuit Failure}

In the simplest digital systems, a clock signal is distributed pervasively throughout the chip to all the internal storage elements. These storage elements are chained together with logic in-between to performs calculations (Figure A.1). When the clock arrives, each storage element takes on the recently calculated inputs from the previous stage. Delays in the clock network create an offset between the various clock arrival times, known as clock skew. The skew causes a stage to trigger before or after it is intended, and thus capture incorrect results, leading to system failure.

\section{A.1.2 Conventional Clock Distribution}

Clock distribution approaches vary, and most often a hybrid of different strategies are used. In any case, the goal is to attain controlled delays throughout the clock network, with minimal overhead in terms of power consumption and area.

Despite propagation delays in clock buffers and wiring, if process and loading across a chip are matched, the clock can be successfully controlled to arrive at all 


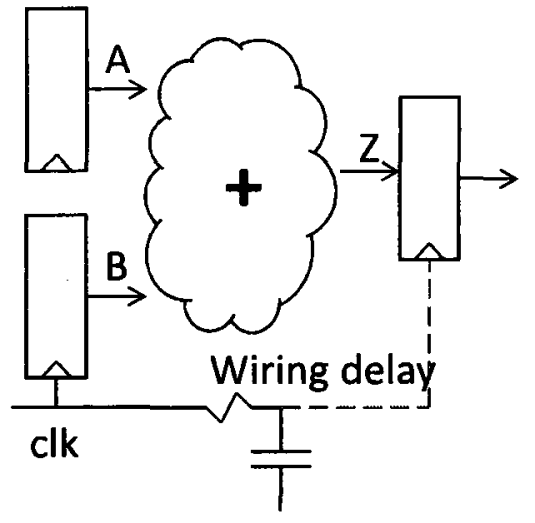

(a) Typical logic circuit

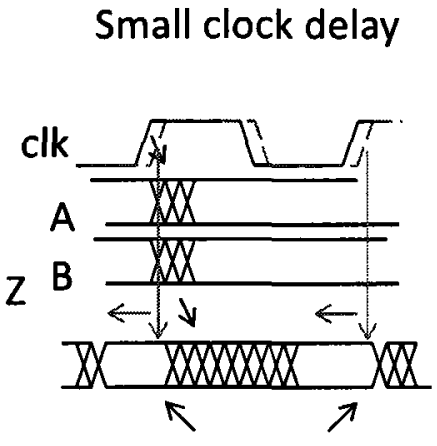

(b) Captures Stable data
Larger clock delay

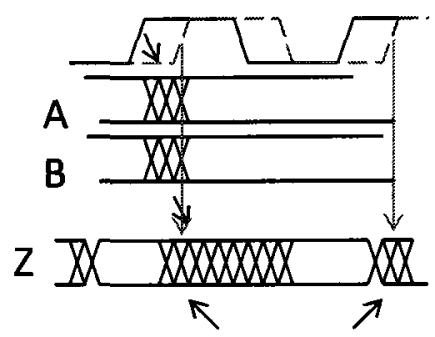

(c) Late clock to Z flop Captures invalid data.

Figure A.1: Typical digital systems consist of chains of registers with logic in-between to perform calculations. When the clock arrives, each register takes on the recently calculated values from the previous stage. In (a), a typical adder circuit is shown, where the output of the logic is $\mathrm{Z}=\mathrm{A}+\mathrm{B}$. The proper timing diagram is shown in figure (b). When the clock arrives, it triggers registers $A$ and $B$ to update their outputs, and $\mathrm{Z}$ begins to fluctuate until the calculation is complete. When the next clock cycle arrives, the stable result is captured in the output register. Panel (c) illustrates what happens if the clock to the output register arrives late. When the clock does arrive, the data has already been released from registers $\mathrm{A}$ and $\mathrm{B}$, and the output $\mathrm{Z}$ is already fluctuating when the register attempts to captures the earlier value. This is referred to as a hold-time violation, since the data was not 'held' fixed at the register $\mathrm{Z}$ input for a suitable margin of time after the clock edge.

flip-flops simultaneously. If the clock is inserted at a central point, and care is taken to ensure that the delay from the source to each flip-flop is identical, then all loads will receive the clock at the same time. Rather than attempt to achieve a 'zero-delay' clock insertion, the goal is to ensure a matched delay to all points in the network. In this way, all loads ${ }^{1}$ receive the clock simultaneously, an insertion delay after the clock was generated.

\section{Symmetric Buffer Trees (H-Trees)}

One of the classic approaches to ensure matched delays to each flip-flop on the chip is through the use of an H-tree (Figure A.2). In this structure, a hierarchical pattern

\footnotetext{
${ }^{1}$ loads, flip-flops, storage-elements, and leaf-cells are all synonymous in this context
} 


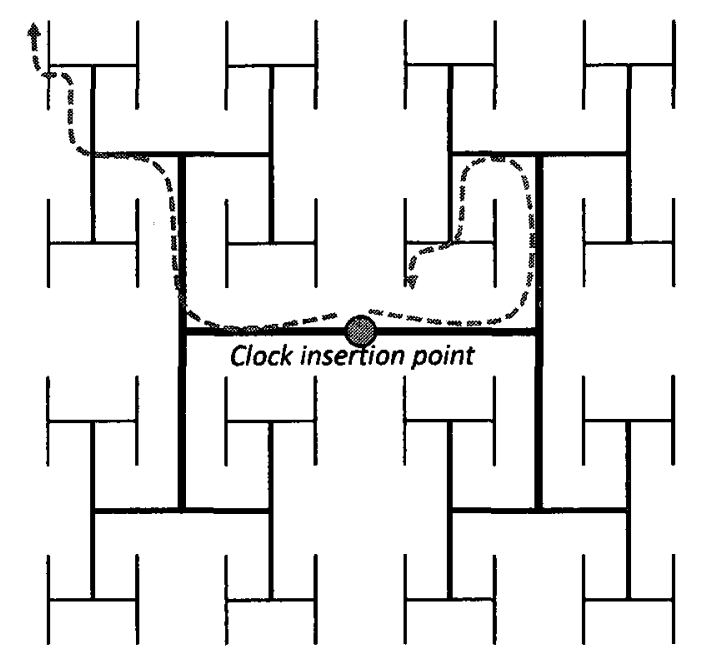

Figure A.2: H-Tree Clock Distribution: Using a symmetric structure such as an 'Htree', the wiring paths are kept identical from the insertion point to each flip-flop in the design. H-trees are well suited to very regular designs, but don't lend themselves to the more typical systems with multiple clock domains.

of $\mathrm{H}$ shaped wiring and buffering is used. The clock is inserted at the center of the $\mathrm{H}$, and propagates with equal delays to all 4 extremities. Then, at these end-points, a buffer is inserted and 4 new $\mathrm{H}$ trees begin. This pattern continues until eventually $\mathrm{H}$ trees at the lowest level are spread throughout the chip and are clocking flip-flops at each of their extremities. The symmetric pattern ensures that the path length from the original insertion point to each flip-flop is identical. As a result, causes of clock skew are restricted to mismatched parasitic loading, and on-chip variations (OCV) due to process, voltage and temperature (PVT) fluctuations.

H-trees work well in regular structures with single clock domains, such as in the clocking backbone of gate-arrays and older FPGAs.

\section{Multiple Clock Domains}

Since beating the clock up and down consumes a great deal of power (it is often estimated at $30 \%$ in digital designs), there is always strong motivation to use a low frequency clock whenever possible. It is typical that only a small portion of a chip will need to operate at high frequency, and it is wasteful to distribute the high frequency 
clock throughout the chip (via an $\mathrm{H}$ tree) when most cycles would be ignored by slower logic.

The trend toward power conscious designs has led to extensive clock-gating, where clock frequencies are selectively scaled or disabled for different portions of a chip. This has led to a proliferation of heterogeneous clock domains. Often at different frequencies, each clock tends to have asymmetric loading and drive requirements. Furthermore, some domains will have loading which is geographically dense and yet others may have the same fanout, yet have loads dispersed throughout the chip. The challenge, is that these dissimilar domains must often be kept balanced to one another and it is prohibitively expensive to build mutually matched geometric $\mathrm{H}$-trees across the chip for small clock domains.

\section{Clustering}

There are a number of electronic design automation (EDA) tools in the marketplace that address the clock distribution of heterogeneous systems. They are based on algorithms which estimate the loading in a particular area of the design and perform first-order parasitic RC extraction for wiring along an anticipated route. Based on these estimates, the tool adds extra buffers, and refines the placement of loads and wiring to match the insertion delay of clocks to one another. It is not uncommon to see these tools insert long strings of buffers in attempts to bring paths into alignment.

Clustering does not give as tight skew control as H-tree systems, but it often works 'well-enough' for the majority of applications. If a designer knows the clock skew is within certain boundaries, he/she can add timing margin into their circuits to guard against the worst possible skew numbers. Unfortunately, the required margin and its associated circuits eat into the available calculation time, and also costs area and power.

\section{Technology Scaling}

As technology scales to smaller geometries, wiring and device variation becomes more significant[31]. The clocks are particularly effected. They operate at the highest speeds, travel the greatest distances, suffer the heaviest loading, require clean sharp edges, and must be synchronized across the chip [32]. 
In $\mathrm{H}$-tree systems, the dominant cause of clock-skew is caused by variations in the clock network's wiring and buffers along what are supposed to be symmetric paths. With clustering, the accuracy of the delay estimates suffer as the wiring and device variability increases. In both cases, worst case skew numbers are increasing.

\section{Increasing Clock Speeds}

Not only is clock skew increasing with smaller devices and poorer interconnect properties, but operating frequencies are also increasing. As such, unintended clock skew consumes a more significant fraction of the overall cycle time [33]. Over a decade ago, Friedman [32] stated, "Performance is limited, not by logic elements or interconnect, but by the ability to synchronize the flow of the data signals." He goes on to say that, "Distributing the clock is one of the primary limitations to building high speed synchronous systems." Partially as a consequence of skew ${ }^{2}$, the clock frequencies of products in the microprocessor market have started to saturate, with performance gains coming about more through parallelism than through brute force speed increases.

\section{A.1.3 Asynchronous Design}

To avoid clock synchronization problems altogether, there are advocates who argue for either asynchronous, or partially asynchronous design. Asynchronous circuits, however, have associated handshaking overhead, and so they often under-perform their synchronous equivalents. Further, simple clocked designs are understood and supported by a larger audience of engineers and electronic-design automation tools, leading to faster project development. For these reasons, Friedman [32] states that "the dominant strategy has been, is presently, and will continue for a long time to be that of fully synchronous clocked systems."

\section{A.1.4 Globally Asynchronous Locally Synchronous Systems}

A compromising strategy to deal with the clock distribution burden is called globally asynchronous, locally synchronous (GALS) communications [34]. In this paradigm,

\footnotetext{
${ }^{2}$ also related to power consumption, heating and wiring
} 
sub-systems are designed conventionally with fully synchronous clocking, and these are then encapsulated with FIFOs and an asynchronous interface which handles the inter-system communications. Since each clock network is independent, and only feeds a small geographically confined area, its skew can be tightly controlled. In the initial stages of this research, the GALS approach was explored and a prototype GALS chip, codenamed Marmoset, was designed, fabricated and tested. Shown in Figure A.3, it was designed to perform general purpose DSP functions for a software defined radio. ${ }^{3}$ After fabrication and testing, it became clear that although the system was functional, the asynchronous message passing formed a bottleneck that limited throughput. Though the IO network could be engineered with more bandwidth, the extra hardware overhead and design complexity were such that they rendered the GALS system less practical than a fully synchronous system. This prototype also contained an array of 15 digitally controlled ring-oscillators of various topologies, which were evaluated in terms of power, area and noise. The results of these oscillator measurements were promising, indicating relatively low cycle-to-cycle jitter (eg. $7 p s_{r m s} @ 300 \mathrm{MHz}$ or $0.002 \mathrm{UI}$ ) for simple single ended CMOS ring oscillators.

Though the oscillator measurements were comforting, the IO speed and interface complexity of the GALS system was disappointing and motivated the return to synchronous systems.

\section{A.1.5 Active Clock Synchronization with DLLs and PLLs}

Referring briefly to the discussion of conventional clock distribution schemes in Section A.1.2, recall that H-tree's tend to be impractical in modern multi-domain systems, and clustering is becoming increasingly inaccurate and inefficient as technologies scale. Clustering is essentially handicapped because it must try to predict the delays of gating cells, buffers, wiring and loading structures in advance - matching the delays of long and very different paths to within a few picoseconds (ps).

Rather than estimate and attempt to balance paths in advance, an active synchronization approach inserts sensors to detect phase offsets, and appropriately tweaks delays to pull clocks into alignment. This approach not only compensates for

\footnotetext{
${ }^{3}$ The system consisted of 8 independent components: 2 filters, 2 arithmetic units, 2 digital sine wave generators, a soft-output error decoding unit (LogMap decoder), and an upconverting DAC.
} 


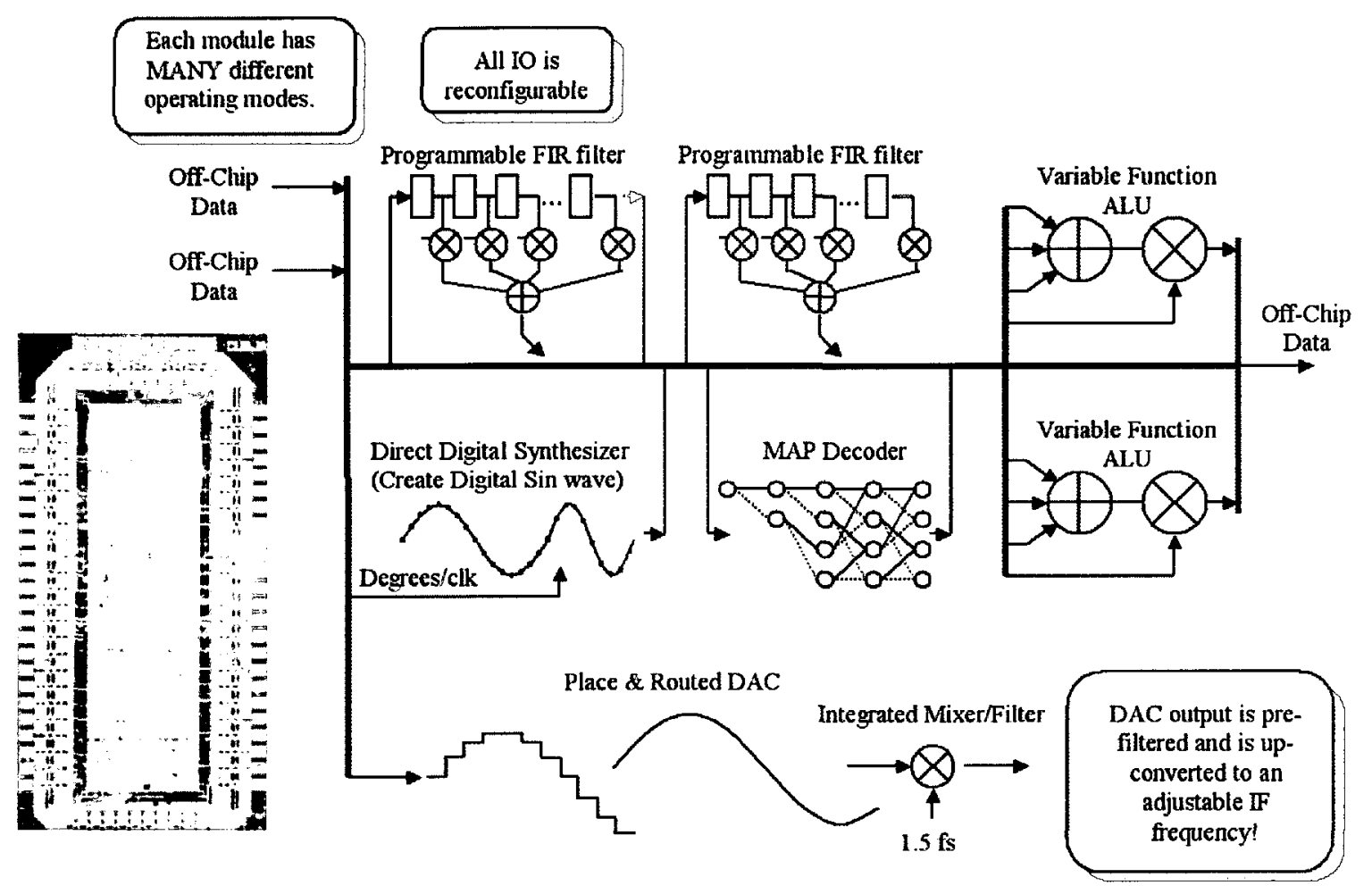

Figure A.3: Marmoset - A Globally Asynchronous Locally Synchronous (GALS) digital signal processing system built early in the research.

static process and load variations which are difficult to accurately predict, but it can also track and remove phase offsets caused by variations in voltage and temperature.

\section{DLL operation and use in clock-skew control}

Two examples of active clock alignment are shown in Figure A.4 [5]. In Figure A.4a, the insertion delay from the global clock to each local distribution grid is tuned to an integer multiple of the clock period. The phase-detector (PD) senses any phase error and the charge-pump (CP) converts this into a current which is averaged by the loop-filter (LF). The resultant voltage adjusts a voltage-controlled delay-line (VCDL) to correct the delay and ensure that $C L K_{\text {ref }}$ is aligned to $C L K_{\text {out }}$. In method b, the system is set up in a daisy-chain, where grid \#1 matches its insertion delay to grid \#2, which matches to grid \#3, etc... At the last grid, the delay-line (and hence 
insertion delay) is fixed to a nominal value, which can be set independently from the clock period.

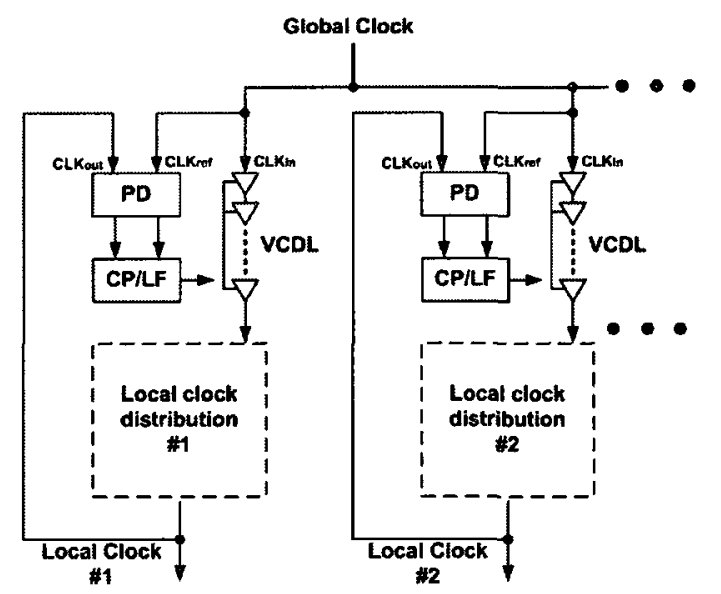

(a)

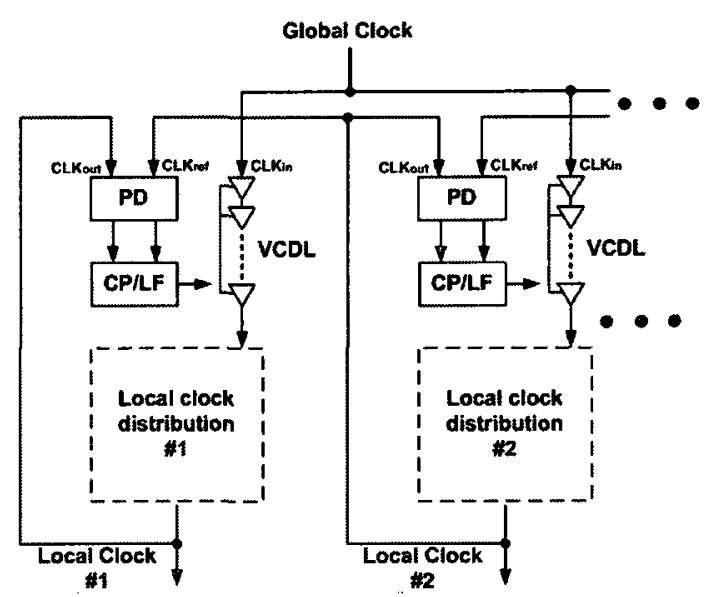

(b)

Figure A.4: Active DLL Clock Synchronization[5] : In method (a), the feedback loop forces the delay through the voltage-controlled delay-line (VCDL) and distribution grid to match an integer number of clock periods. This ensures that the output grid is aligned to the reference port regardless of loading, process variations or temperature. In method (b), the clock grids are connected in a daisy-chain: grid \#1 is synchronized to grid \#2, which is synchronized to grid \#3, etc. In the final stage the last grid would be matched to a nominal delay element (which can be less than one period of delay). When the DLL does not need to maintain $2 \pi$ of phase-shift through the delay-line, as in this case, it will be referred to as a 'deskewing' DLL. Since short delay-lines (with low absolute delay) can be used, deskewing DLLs suffer less peak-to-peak jitter due to noise sources.

\section{PLL operation and use in clock frequency and skew control}

As an alternative to the DLL distribution schemes typified by Figure A.4, a PLL based system is shown in Figure A.5. The PLL, which will be more thoroughly described in Chapter 2, also detects phase-error, but it uses this information to control an oscillator instead of a delay line. The clock generated by the voltage-controlled oscillator (VCO) is controlled by the feedback loop so that it is aligned to the reference clock, and so the PLL can also be used for clock alignment. Unlike most DLLs however, the PLL typically generates a higher output frequency than input frequency. 


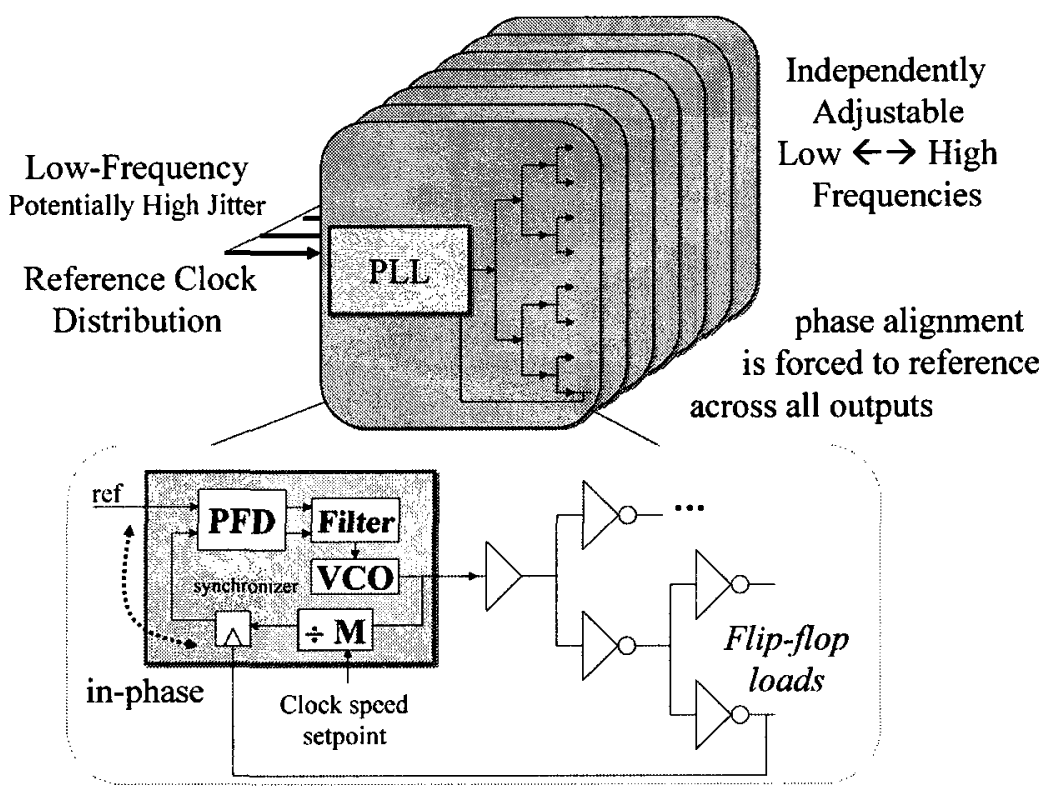

Figure A.5: PLLs for Clock Synchronization and Frequency Control: Like a DLL, a phase-locked loop can be used to synchronize the output of a clock-tree to a reference input. A phase/frequency detector (PFD) senses any phase error between the arrival time of its inputs, and through a filter structure, generates a signal which adjusts a voltage controlled oscillator (VCO). The oscillator then goes through a divider for presentation to the PFD. Since the feedback will work to keep both inputs to the PFD at the same phase and frequency, the VCO output frequency will be Mx the reference frequency. While the PLL is more complex than a DLL, it has the advantage that it can easily generate multiples of the reference frequency for different parts of the chip. Since the output clock is aligned to the reference, it facilitates communication between sub-systems clocked at different rates.

Rather than distribute a high-frequency clock at considerable expense, power and complexity, a low-frequency clock can be distributed to regional PLLs. In turn, each PLL independently clocks its leaf nodes at an appropriate frequency. In addition to power savings, localized speed control also improves system flexibility, simplifying integration of circuits with different critical paths. Another significant advantage is that the loop controls the output clock phase to match the reference port, with only a slight predictable offset. This permits synchronous IO between logic islands clocked at the same or different frequencies.

Both the DLL and PLL based approaches compensate for local loading, supply, and PVT (process/voltage/temperature) variations, which are the dominant cause of 
clock skew [32]. They therefore synchronize clocks far more accurately than clustering methods, or even symmetric buffer trees. 


\section{Appendix B}

\section{Further Simulation Results}

\section{B.1 Overview}

This section includes simulation results which support the data found in earlier chapters.

\section{B.2 Charge Pump}

\section{B.2.1 Noise of the PFD, Prefilter and Charge-Pump}

Periodic-Steady State (PSS) and Periodic Noise (pnoise) simulations were done to characterize the noise contributions of the cascaded PFD, prefilter and charge-pump. Often, these sources dominate the noise at offsets close to the carrier (in-band), where the VCO noise is being suppressed. The result of these simulations is shown in Figure B.2.

Of particular importance, the inactive nodes of the CCP are not subject to modulation and are insignificant contributers. In this particular case, the dominant noise source is the flicker noise of the slow turn-off transistors in the prefilter. This makes intuitive sense because these noise sources are multiplied by the gm of the charge-pump transistors before making it to the output node. The prefilter schematic is shown in Figure B.3. If designing for improved in-band noise performance, the size of these transistors would be significantly increased to reduce their impact. In this application low-power was the primary consideration, and their size impacts the drive and current requirements of the PFD slightly. 
The noise out of the cascade is plotted in $A / \sqrt{H z}$. This noise can be input referred by dividing it by the effective charge-pump gain, which in this case depends on the operating region. For very small phase errors the pump gain is approximately $1 \mathrm{~mA} / 2 \pi \mathrm{rad}$, yielding an input referred noise from the 'active' node of $-230-20 \log (1 \mathrm{~m} / 2 \pi)=-154 d B c @$ a $10 \mathrm{kHz}$ offset. Note that this node is responsible for $44 \%$ of the noise, and so the total input referred noise from the pump would be $\approx 6 d B$ higher at $-148 d B c @ 10 \mathrm{kHz}$ offset. When multiplying by 32 , this noise is transferred to the output with a penalty of $20 \log (32)=30 \mathrm{~dB}$, and so we would expect no better than $-118 d B c / H z$ due to pump noise. For larger steady-state phase errors, the pump gain drops to $\approx 175 u A$ and the output referred noise degrades to $-102 d B c / H z$.

While the prefilter dominates the noise performance, a legitimate question is how far down is the contribution from the charge-pump transistors themselves (those in the tri-state gates)? Figure B.4 shows the contribution from the charge-pump transistors becomes significant at about $10 \mathrm{MHz}$.

\section{B.3 VCO Design, Range and Noise Characteriza- tion}

The VCO used for this design is a pseudo-differential ring-oscillator.

\section{Power and Area}

The primary requirements for this design are low power and area. There is a tradeoff between these goals and low noise, since larger transistors lead to better signal-tonoise ratios. In a ring-oscillator stage, for example, delay $\propto C * V / I_{d s}$, where $C$ is the capacitance, $V$ is the voltage swing, and $I_{d s}$ is the transistor's effective drainsource current. Junction noise in a transistor is proportional to the $\sqrt{I_{d s}}$, but delay is proportional to $I_{d s}$ itself. Since signal grows faster than noise, larger currents can be used (and offset with higher capacitance to maintain the same delay) to make the stage less sensitive to noise. Flicker noise also benefits from larger devices, where the flicker co-efficient of a transistor is derated by the area of the gate. 


\section{VCO Noise}

In many cases where a ring-oscillator is used, it is the dominant noise contributer and a wide loop bandwidth must be used to keep it under control. In this case, the pump noise has been predicted from simulations to be between $-102 \mathrm{dBc} / \mathrm{Hz}$ to $-118 \mathrm{dBc} / \mathrm{Hz}$ (depending on the phase error and thus pump gain) @ $10 \mathrm{KHz}$ offset.

\section{B.4 Filter Construction}


Charge into Filter vs Phase Error (Response of Phase Detector + Thermometer Filter)

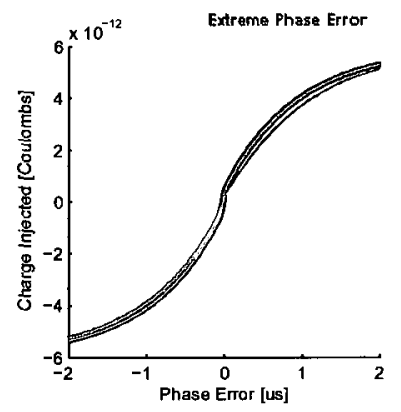

Legend

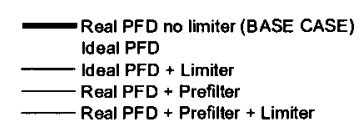

$\cdot$
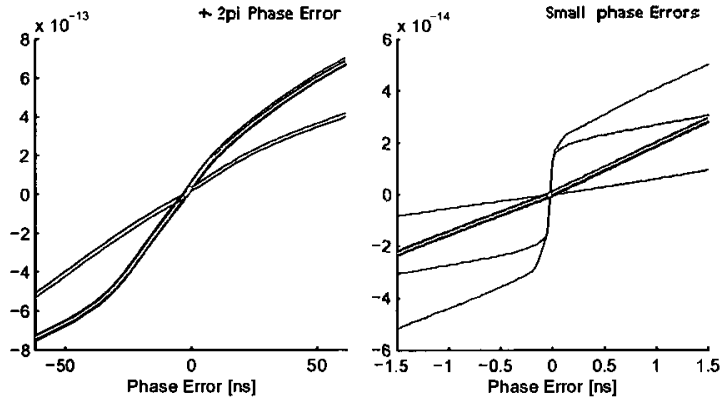

Linearity Error
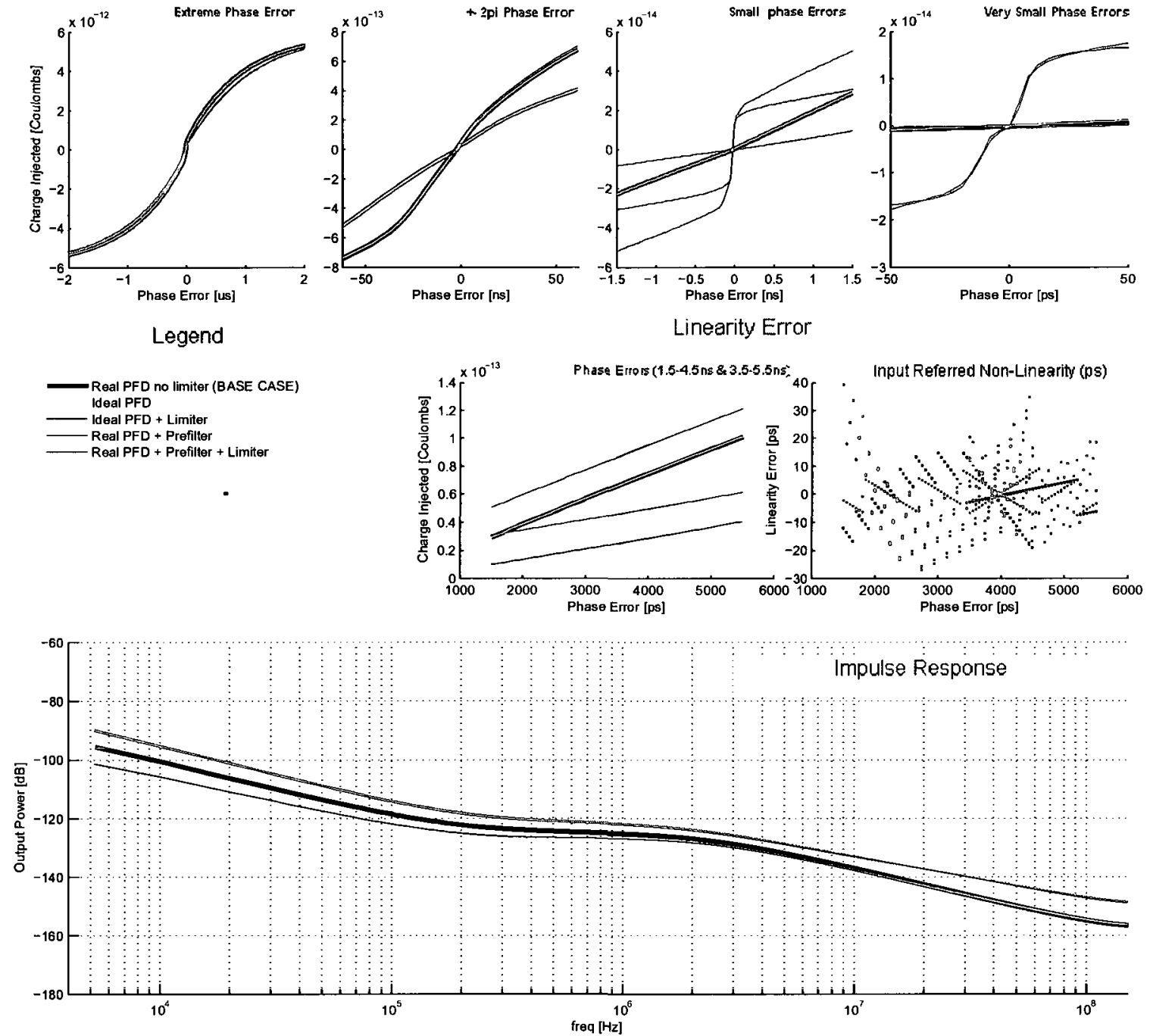

Figure B.1: Prefilter and Charge-Pump Response versus Phase-Error The top plots show the charge integrated by the cascaded charge-pump and filter for different ranges of phase-error. The curves on each plot compare real and ideal PFDs, and circuit with the pre-filter and limiting circuitry on or off. The prefilter causes significant bends in the curve since it intentionally exaggerates small phase errors. Below $\approx 20 \mathrm{ps}$, it increases the effective pump current from $\approx 175 u \mathrm{~A}$ to $>1 \mathrm{~mA}$. The second set of plots show the deviation of the characteristic from a best-fit linear curve (for phase errors between $1.5 \mathrm{~ns}$ and $5.5 \mathrm{~ns}$ ). This operating region is away from the non-linear portion of the prefilter and so its input referred non-linearity is not significantly degraded compared to the other cases. The bottom panel shows the impulse response of the cascade. Note that it has the expected response discussed in Chapter 2 with a low-frequency pole near $\omega=0$, a zero at $1 / R C_{1} \approx 200 k \mathrm{~Hz}$ and a higher order pole at $1 / R C_{2} \approx 2 M H z$. 

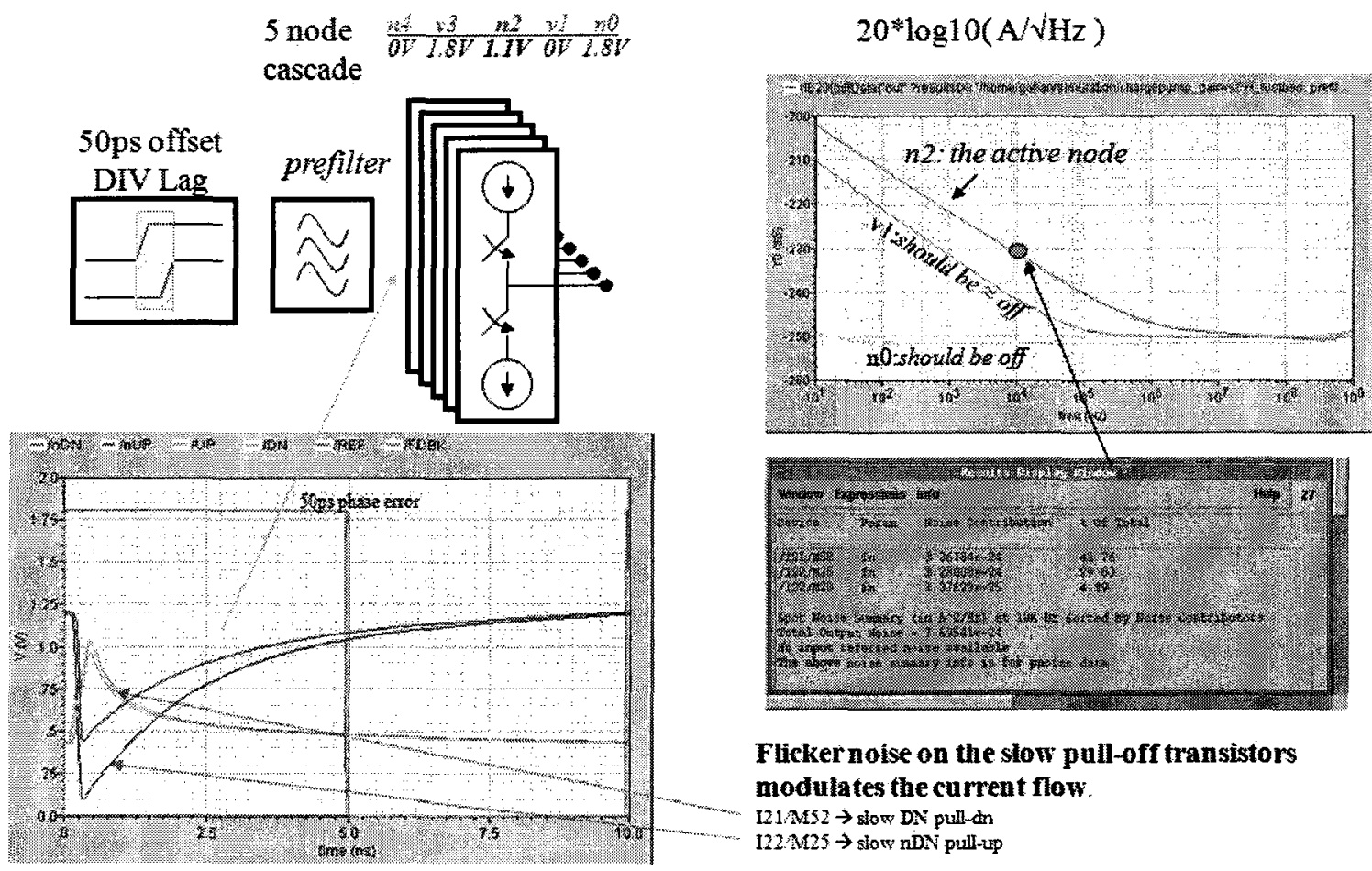

Flicker noise on the slow pull-off transistors modulates the current flow.

DiM $12 \rightarrow$ slow DA pull-dn

$122 / 2525 \rightarrow$ slow nDN pull-up

Figure B.2: Periodic-Steady State (PSS) simulation results of a cascaded PFD, prefilter and charge-pump. A 50ps phase error is introduced into the chain and is acted upon by the prefilter to produce control voltages to the cascaded charge-pump (UP, $\mathrm{DN}$, and active low versions $\mathrm{UPb}$ and $\mathrm{DNb}$ ). In the bottom left pane, the eye-diagram of the PSS simulation shows how the 50ps phase-offset is converted into a drawn-out control voltage difference between $\mathrm{UPb}$ vs $\mathrm{DNb}$ and UP vs DN. The cascaded chargepump uses this difference to regulate current flow. Since a short duration pulse is extended into a longer duration one, the current driven by the charge-pump can be of lower amplitude (for a longer duration) while still maintaining the same pump-gain. The noise plots show the total contributions on VCO control nodes n0, v1 and n2. As expected, with $\mathrm{n} 2$ in the analog range and subject to modulation, it contributes the most noise. The neighboring signal is 'slightly' on, and contributes $10 \mathrm{~dB}$ less noise, and the signal 2 nodes away from the transition point of the code (n0) contributes nothing. 

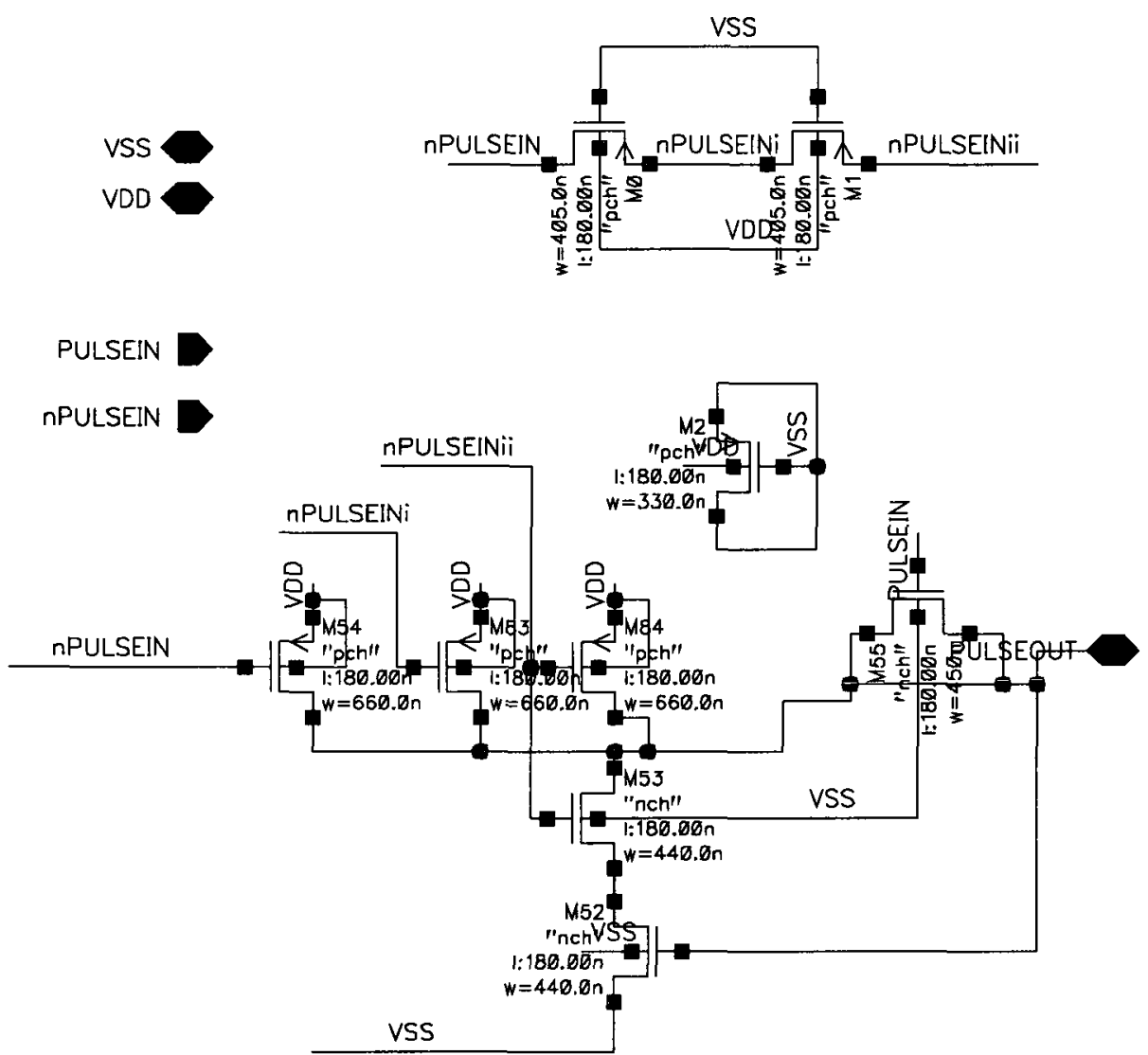

Figure B.3: Prefilter and Charge-Pump Noise Contributers: The primary noise contribution within the PFD/CP chain (73\%)is the flicker noise of the transistors in the pre-filter which modulate the control signals to the cascaded charge-pump. 


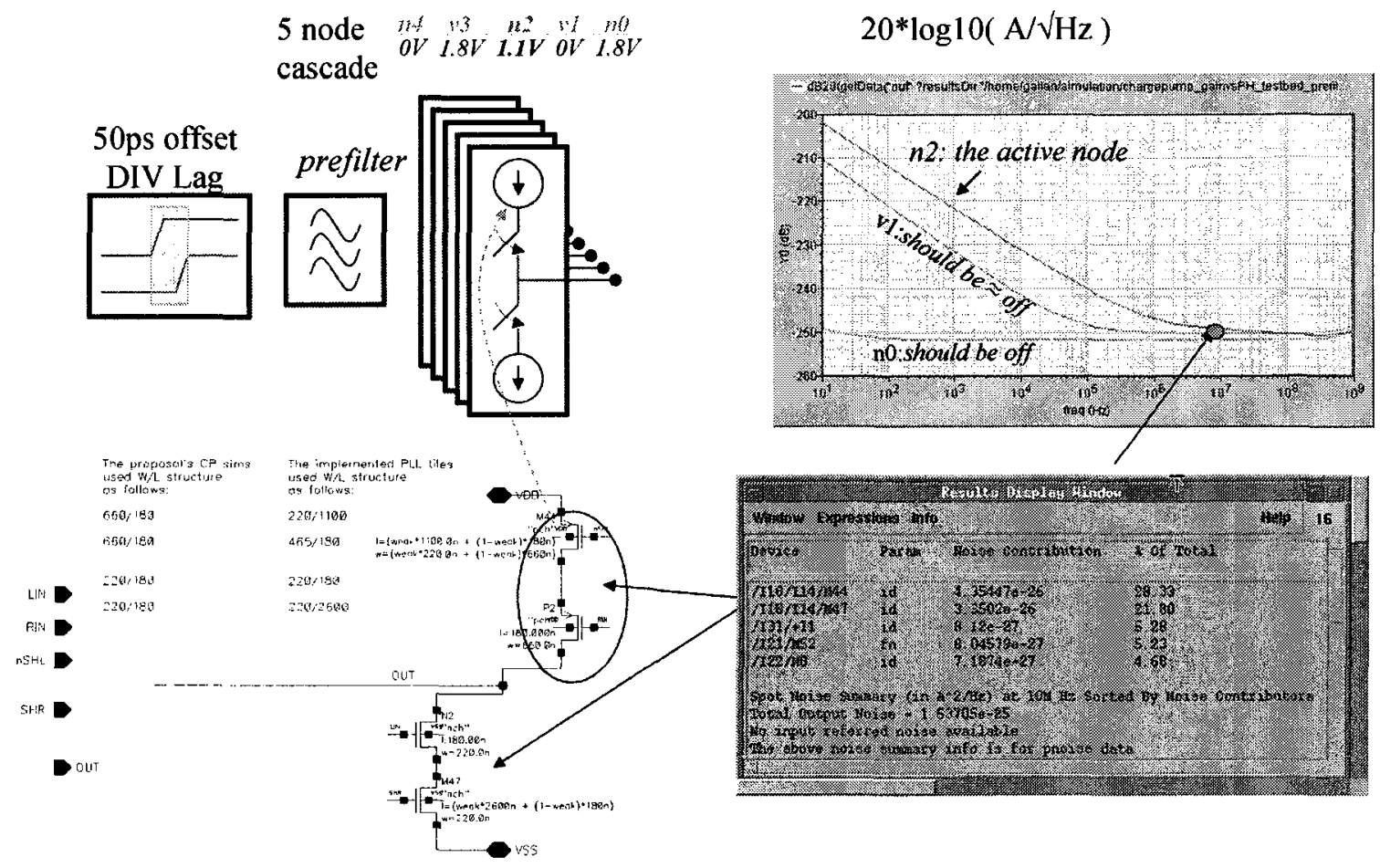

Figure B.4: Noise from CP Transistors themselves becomes significant at $10 \mathrm{MHz}$ offset 

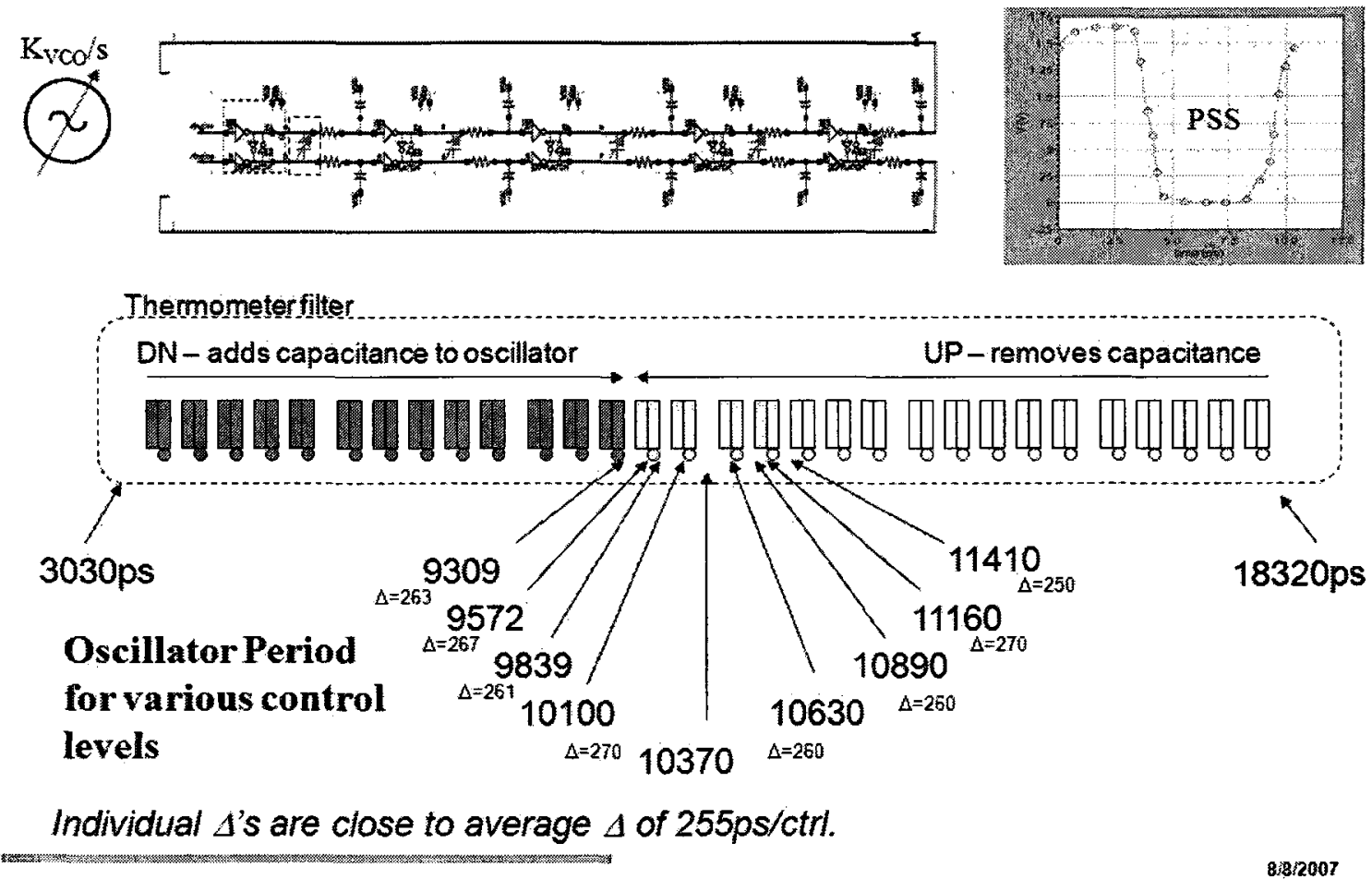

Figure B.5: A Pseudo-differential VCO was used with a range of $3030 \mathrm{ps}(330 \mathrm{MHz})$ to $18320 \mathrm{ps}(54.6 \mathrm{MHz})$ under typical conditions. To modulate the frequency, capacitances are exposed between the positive and negative branches of the ring. 
Back-annotated wiring parasitics

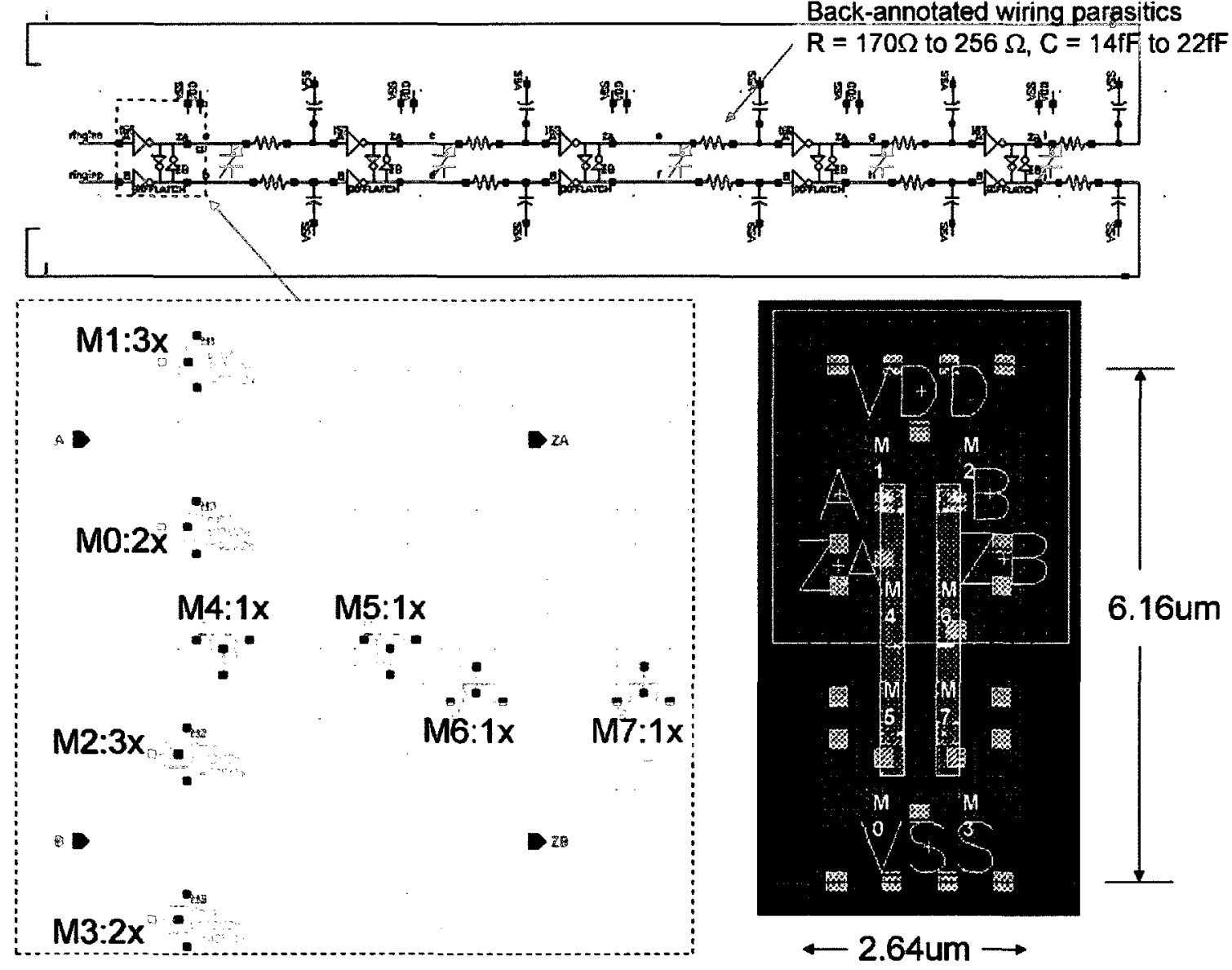

Figure B.6: VCO Stage Details 


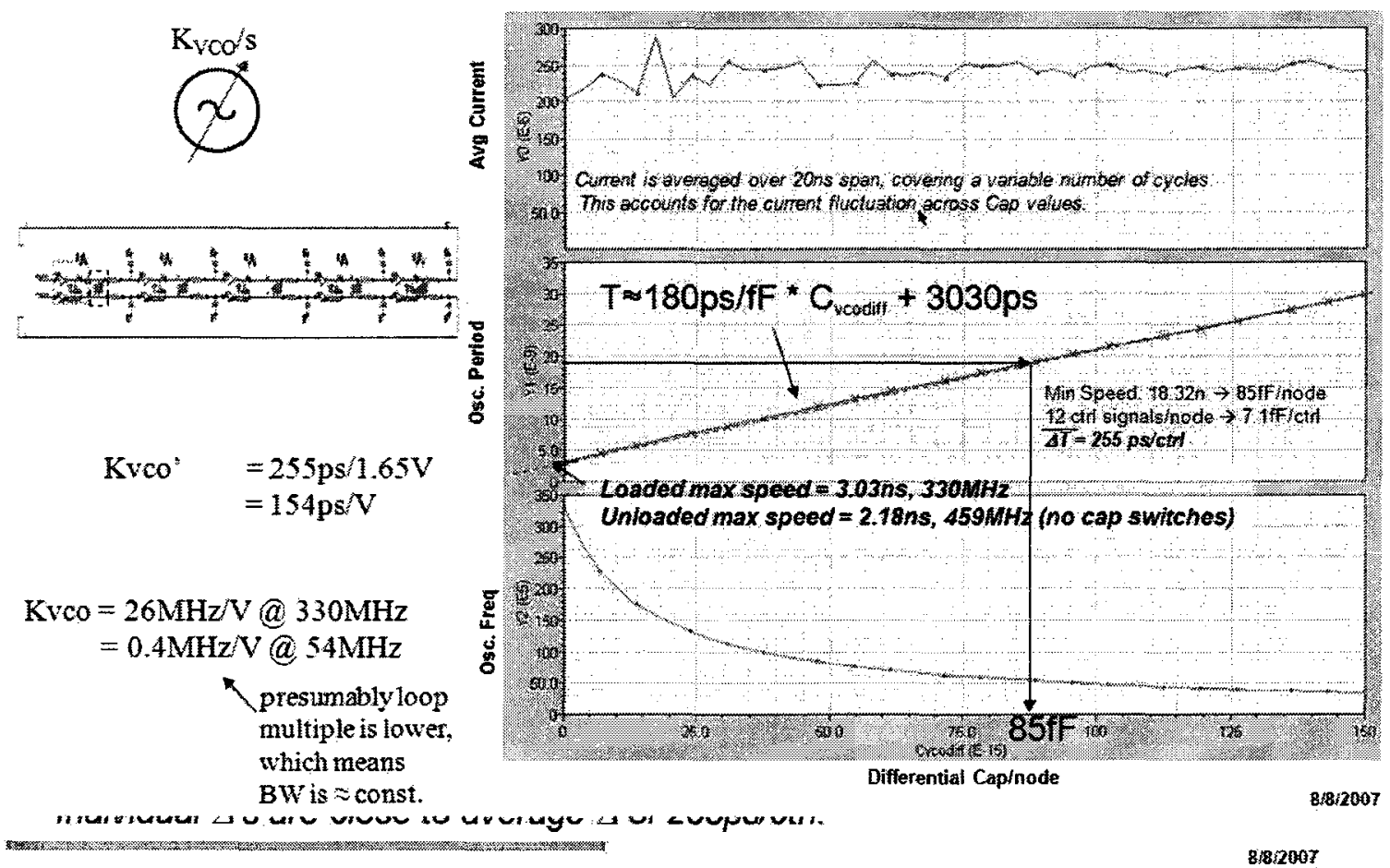

Figure B.7: Power consumption of the VCO 

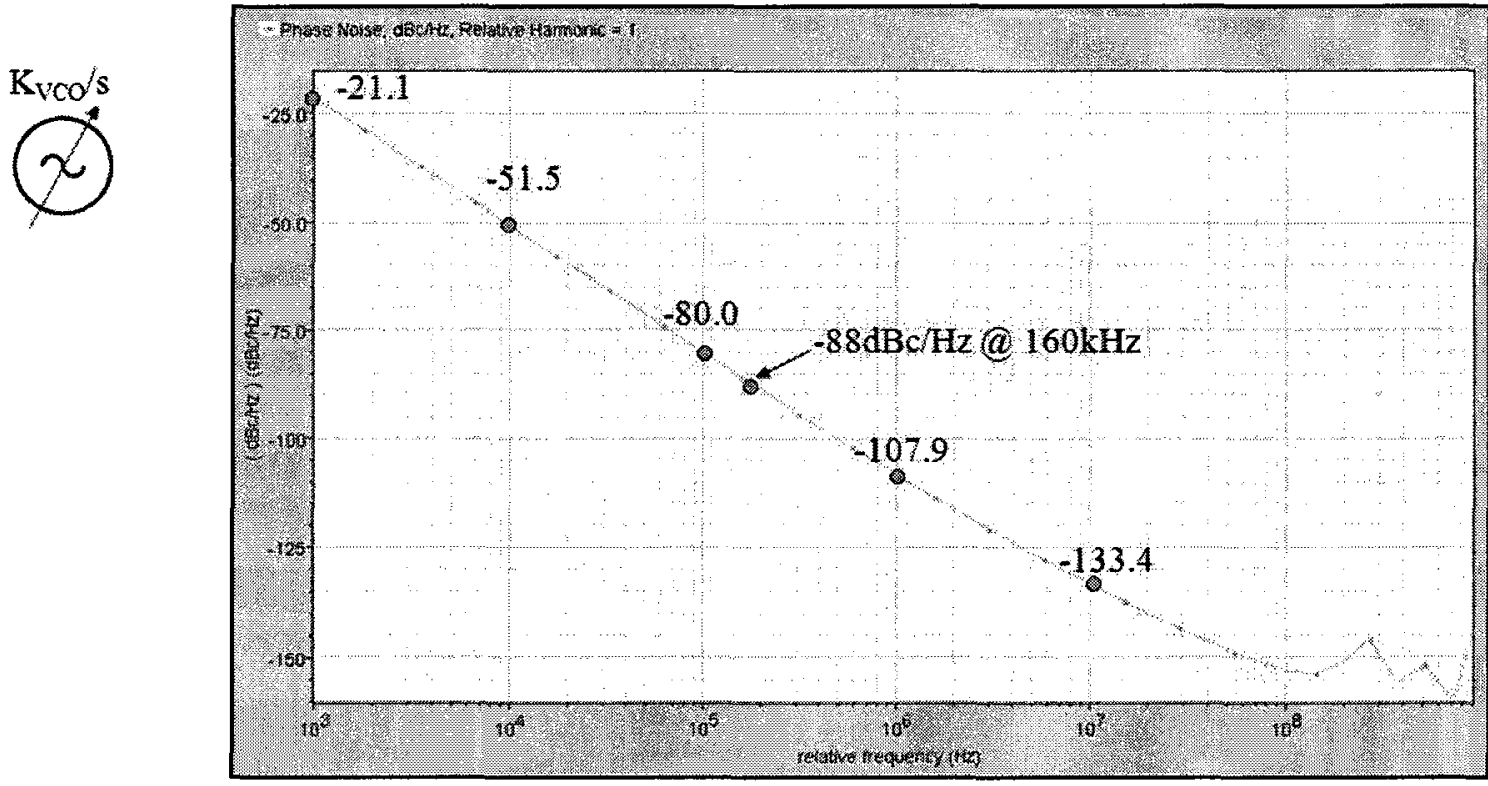

PNoise Simulation: Noise contributors $1 \mathrm{kHz} \rightarrow 1 \mathrm{GHz}$ $T=27 \mathrm{C}, 1.65 \mathrm{~V}$, typical, freq setting for $125 \mathrm{MHz}$ 10 sidebands

Figure B.8: Phase Noise of the VCO 


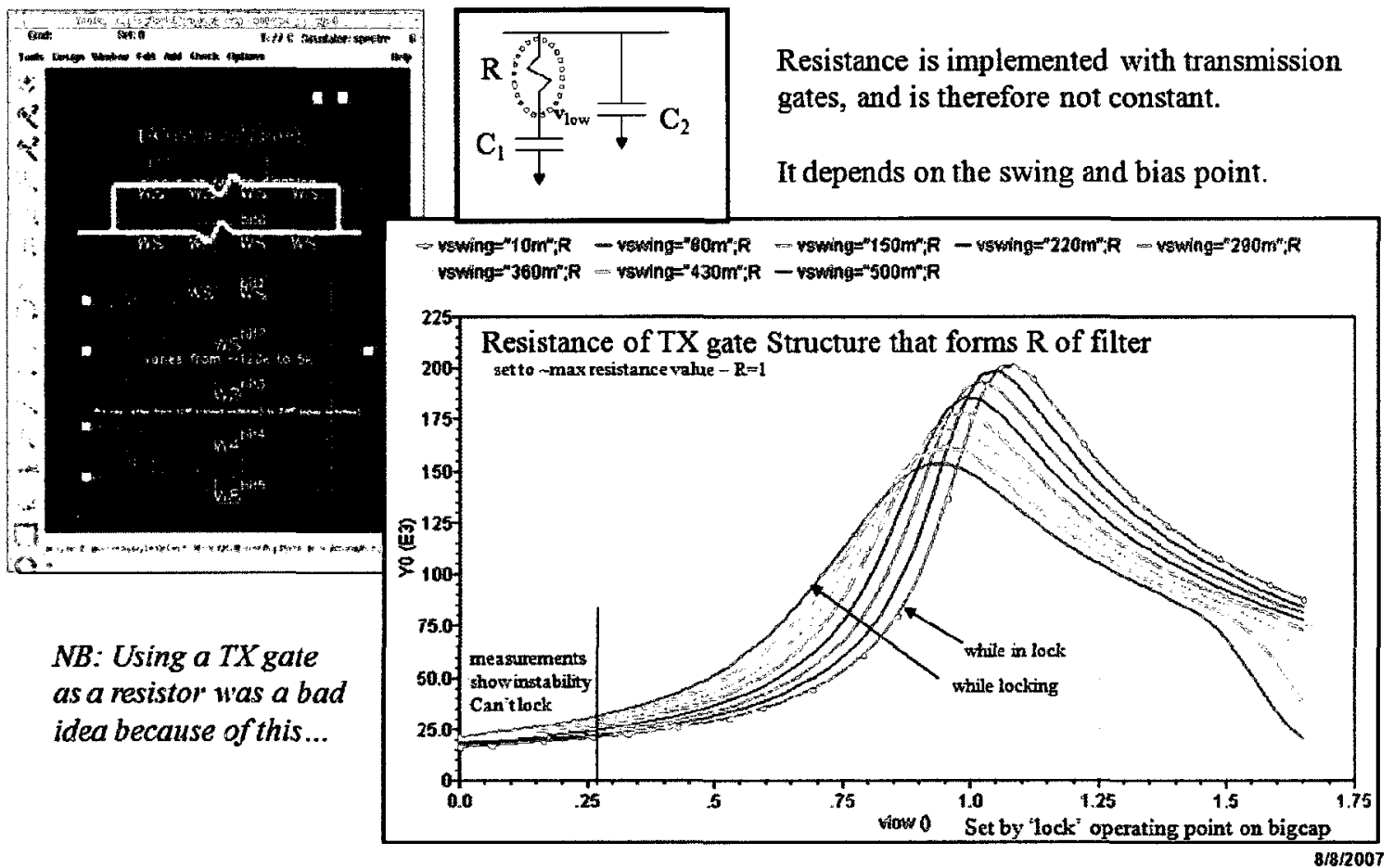

Figure B.9: Characterizing the Resistance of Transmission gates used for filter ' $R$ ' 

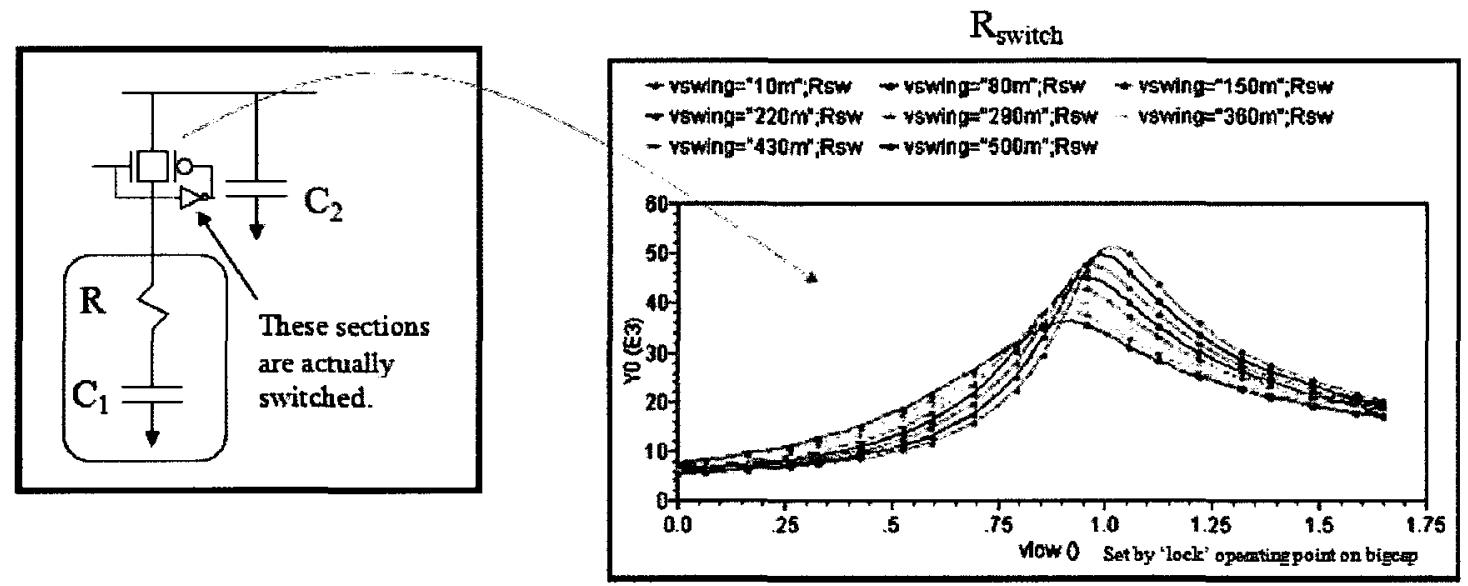

Resistance in the filter domainates over the resistance of the transmission gate switch.

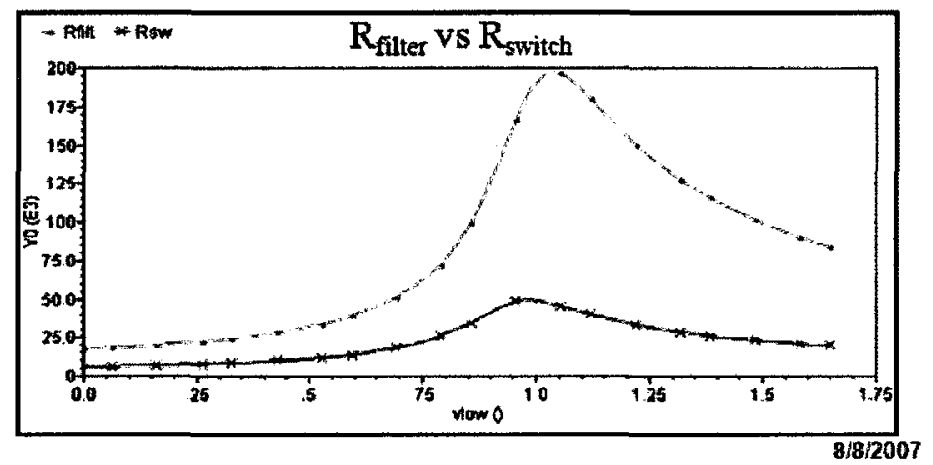

Figure B.10: Resistance of the intentional TX gate ' $R$ ' should dominate over the switch used for filter rotation in the charge-pump structure. 

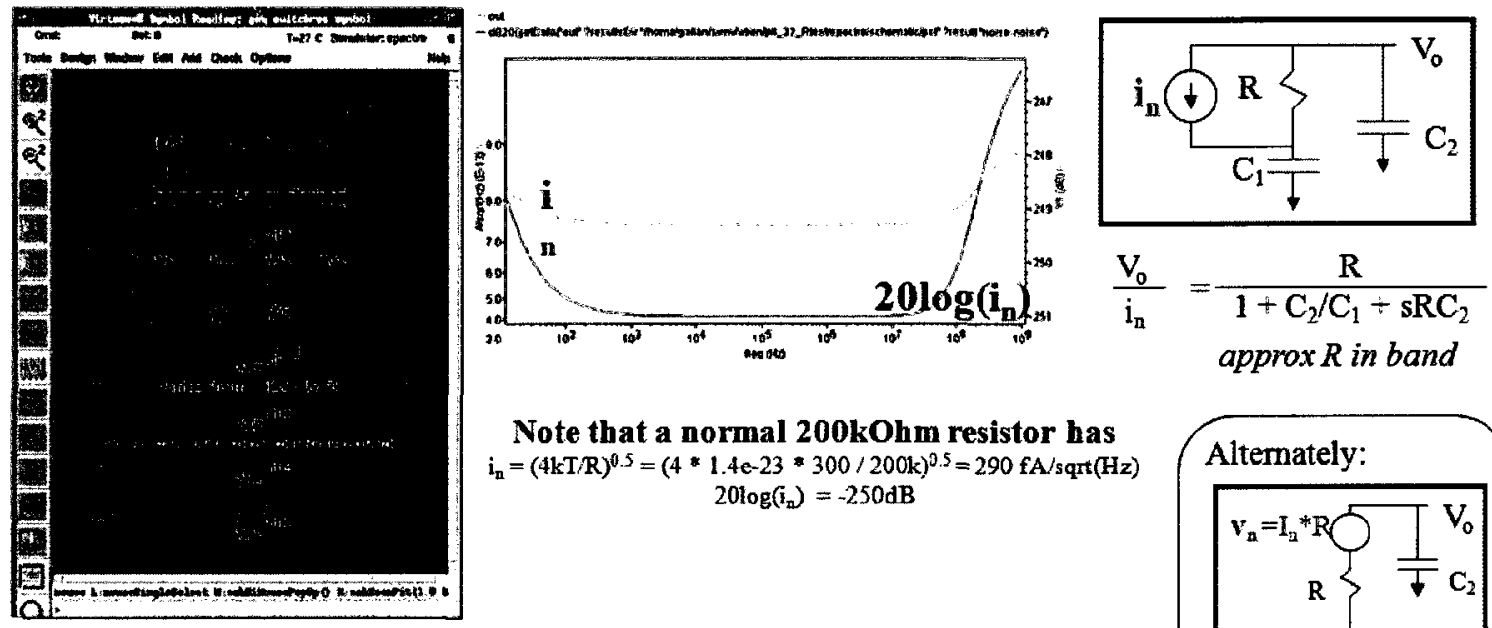

Note that a normal $200 \mathrm{kOhm}$ resistor has $i_{n}=(4 k T / R)^{0.5}=(4 * 1.4 \mathrm{e}-23 * 300 / 200 \mathrm{k})^{0.5}=290 \mathrm{fA} / \mathrm{sqrt}(\mathrm{Hz})$ $20 \log \left(\mathrm{i}_{\mathrm{B}}\right)=-250 \mathrm{~dB}$

Biased w/ $5 \mathrm{mV}$ across $\mathrm{R}$

Very little current, low flicker noise.

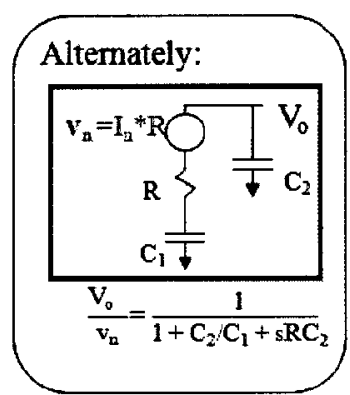

Figure B.11: Noise of Transmission gates within the Cascaded Charge-Pump: Since there is very little current traveling through the filter at any time, the noise is relatively low.
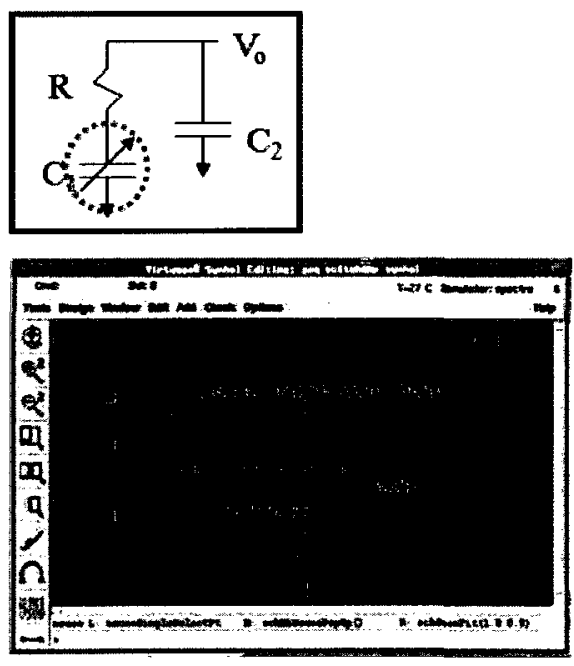

Switched MOS caps work reasonably well.

The deviation across voltage can get up to $35 \%$ though... Not nearly as bad as the $R$ variation of the TX gates setting

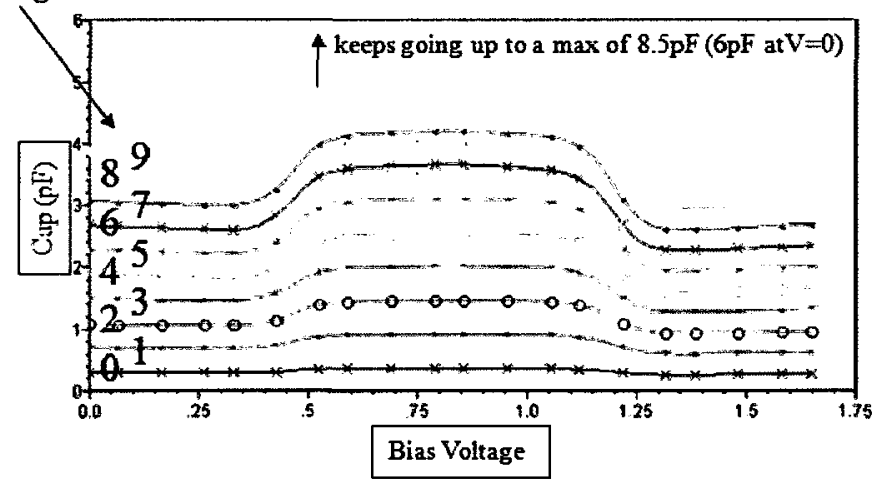

Figure B.12: Capacitance variation of MOS caps vs bias voltage 


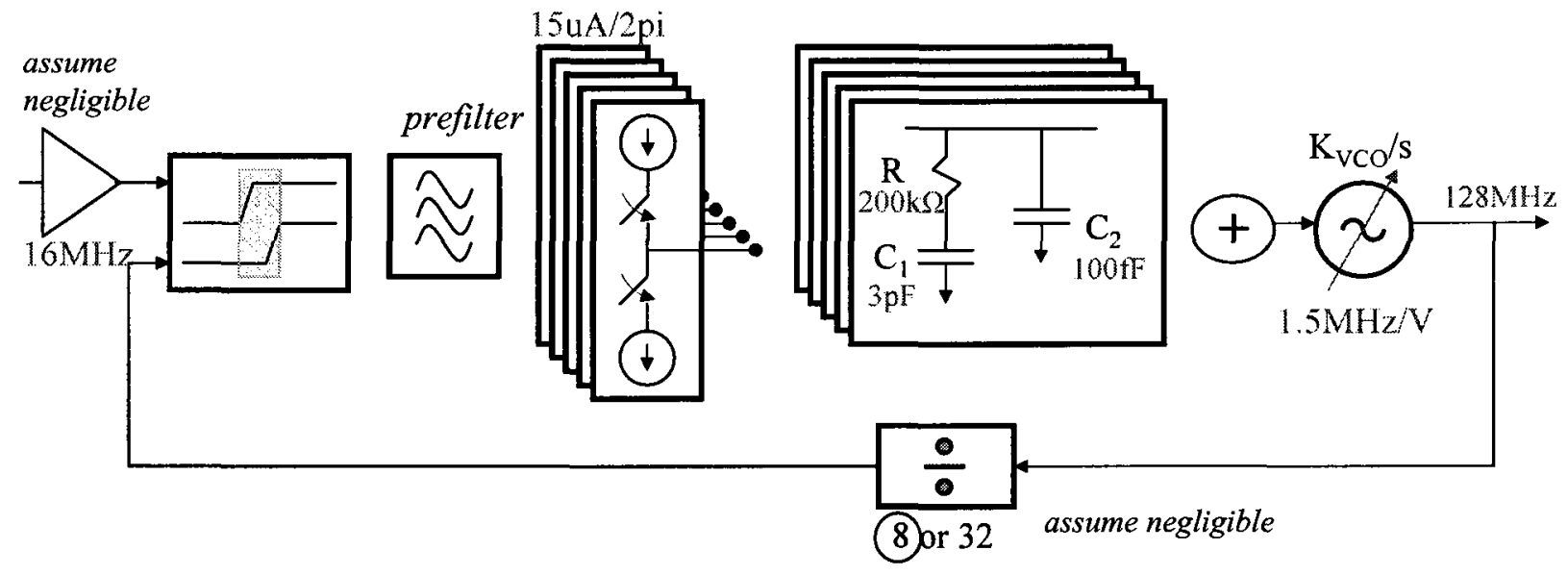

Figure B.13: Component Integration 

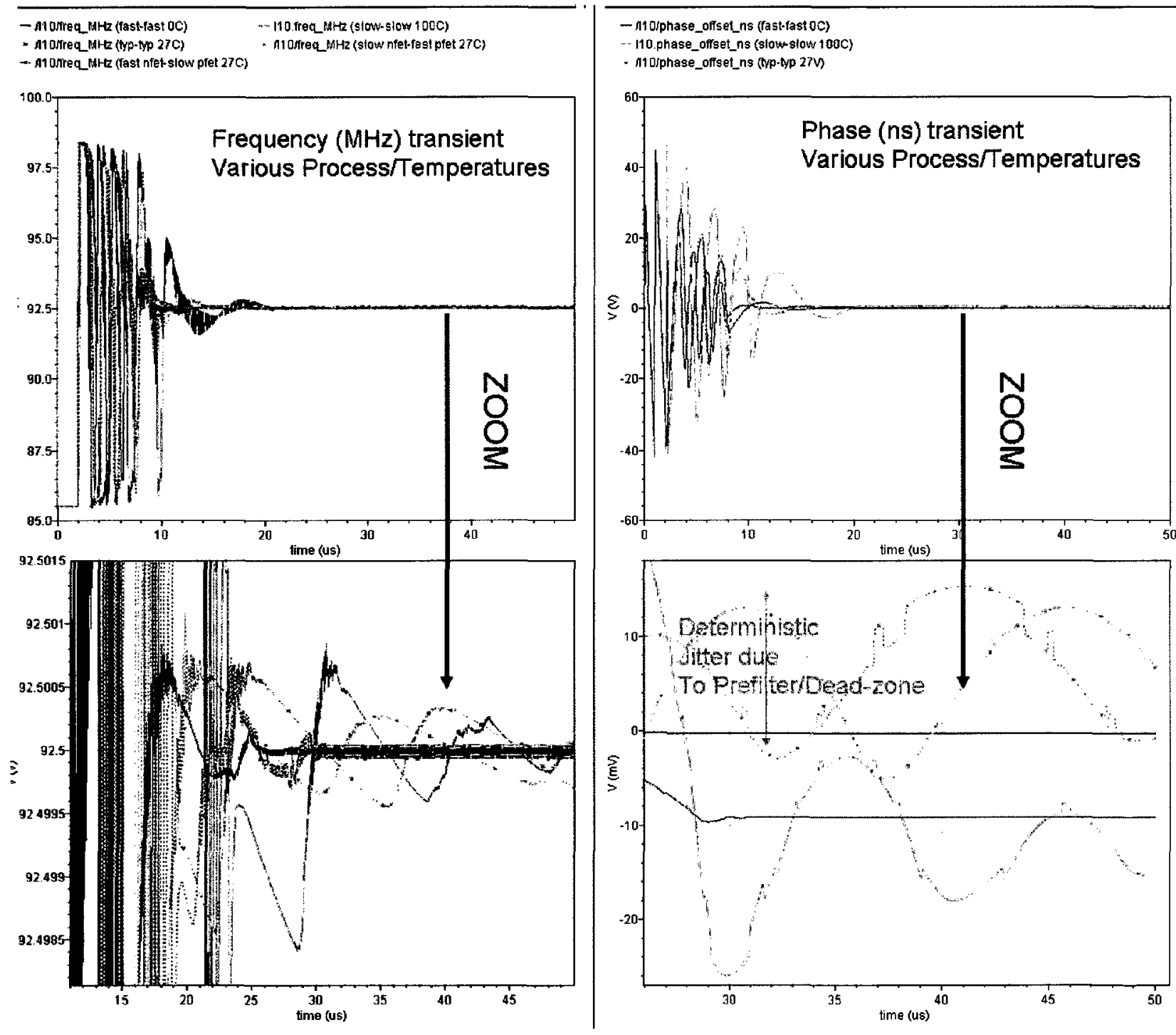

Figure B.14: Simulated Locking under various Process/Temperature Conditions 


\section{Appendix $\mathrm{C}$}

\section{General PLL Design Procedure}

Depending on the starting point, the design procedure for a PLL will vary. For example, the starting point may be a phase-noise mask, jitter specification, current limit, lock-time requirement, area requirement or any weighted combination.

For the procedure outlined below, it will be assumed that the user begins with a phase-noise mask and a directive to minimize area and power while meeting the phase-noise specification.

Outside the loop bandwidth, the noise is dominated by the VCO whereas inside, it is typically dominated by the charge-pump. At the moment, lets assume the designer is given some flexibility to chose the $\mathrm{BW}$ which minimizes total noise as long as the mask is met. Before the VCO and $\mathrm{CP}$ is designed, however, the optimal BW for noise suppression is unknown. As a starting point, the designer asserts that the BW will lie somewhere between $30 \mathrm{kHz}$ and $1 \mathrm{MHz}$. The VCO design can proceed, focusing on meeting the phase noise mask $>1 \mathrm{MHz}$, while the CP design focuses on meeting the mask $<30 \mathrm{kHz}$. Refinement of each design may be necessary once the final loop BW is chosen, and the two components are mixed together.

\section{C.1 VCO Design}

If out-of-band noise specifications are relaxed, a ring-oscillator is a good choice due to its small size and good efficiency. Quick phase noise simulations can be done on both a minimally sized 5-stage inverter ring and one with much larger transistors (eg. $\mathrm{W}=100 \mathrm{x}, \mathrm{L}=5 \mathrm{x})$ to provide reasonable bounds on achievable phase noise. The larger transistors consume more power, have lower flicker noise, and drive larger currents - making them less susceptible to junction noise which only grows with $\sqrt{I_{D S}}$. The 
smaller transistors consume less power and area but are more susceptible to noise and circuit parasitics. Capacitance can be added on each node of the oscillator to tune down the ring oscillation freq. and match the expected VCO center freq. For low frequencies where the rise/fall times of the inverter stages becomes quite large (eg. $20 \mathrm{x}$ a gate delay in a given technology) or the load capacitors become quite large, the designer may consider a $\mathrm{VCO}$ which naturally runs at a higher frequency and couples to a divider at the output.

If the ring-oscillator 'bounding' simulations show that the out-of-band phasenoise specification is achievable, size down the transistors from the low-noise scenario (while sizing the load capacitor to keep freq $\approx$ constant) until the out-of-band phasenoise mask is met, with a few $\mathrm{dB}$ of margin. This will keep the VCO power and area consumption down.

Thus far, the oscillator is not controllable. To modulate it, there are two main options: 1) change drive strength, 2) change loading. It is easier to achieve large frequency variation (high $K_{V}$ ) by changing the drive strength, but the noise is primarily a factor of transistor drive, and so the phase-noise will vary with lock position. The second option involves substituting some of the fixed capacitive load for varactor stages on each node of the oscillator. The varactor can be made using NMOS or PMOS transistors, where the gate bias is modulated, and the drain/source are tied together to the load-line of the oscillator. Normally the required $K_{V}$ is fixed by the required frequency range (which can sometimes be a single point). It is necessary to cover the required frequencies of operation across process/voltage/temperature (PVT) fluctuations. Simulations across corners can be used to determine the overall $K_{V}$, and the ratio of fixed to varactor capacitance. The varactor substitution should be done, and the VCO resimulated to check and iterate against any degredation in phase-noise.

If using the cascaded charge-pump advocated in this thesis to minimize circuit size and improve phase-noise, then the control to the VCO will be vector of signals. It makes sense to distribute the varactor (or other) controls in a 'round-robin' fashion to the various nodes of the oscillator to avoid heavily loading one node in favor of the others. 
Once the VCO is coupled with the charge-pump and a bandwidth is chosen, further refinement of the transistor sizes can be done to minimize power or noise while meeting the phase-noise mask.

\section{C.2 PFD}

As with the VCO, the PFD and CP design can start by performing some basic simulations of some bounding scenarios. A standard dual flop-flop PFD with a few gates of delay in the reset path can provide realistic UP/DN signals to the chargepump. The charge-pump noise will tend to be dominated by a combination of the current sources, switches and phase-detector jitter.

A good starting point is to determine the noise contribution due to the jitter of the phase-detector itself. Start by coupling the UP/DN control signals from a minimally sized PFD, though some buffer stages, to ideal current sources/sinks and switches, and then into an ideal voltage source. At this stage, the current/gain of the ideal charge-pump will not effect the simulation results, but you may wish to use realistic numbers in preparation for when the charge-pump is swapped with a real charge-pump. Keep in mind that the PFD buffer stages will eventually need to drive the switches of the charge-pump. We don't know how big these are yet, but we can start with an assumption of 10x output stage buffers, and refine this later.

A periodic-steady-state (PSS) and periodic noise (pnoise) jitter simulation can be done using SpectreRF to simulate an output noise spectrum, in $A m p s / \sqrt{\mathrm{Hz}}$. Since the charge-pump is ideal, this noise is due to the digital jitter of the PFD/buffers. Divided by the ideal charge-pump gain $A / 2 \pi r a d$, and taking $20 * \log ($ ans $)+20 \log \left(f_{v c o} / f_{\text {ref }}\right)$, produces the scaled spectrum in $d B c / H z$ at the VCO output. To ensure that the PFD won't be a significant contributor to charge-pump noise, selectively size up the transistors on the signal path (inside the flip-flops) and subsequent buffer stages, until the PFD contribution is $\approx 10 \mathrm{~dB}$ below the noise-mask at frequency offsets below the maximum potential loop BW. 


\section{C.3 Charge-Pump}

The analog current sources of the charge-pump are typically the dominant source of in-band noise and will be tackled next. As with the VCO, if currents go up by $4 \mathrm{x}$, noise only tends to go up by $2 \mathrm{x}$, and so a net improvement is achieved with higher pump currents. In addition to the obvious cost (more power consumption), higher currents require larger transistors (more area) and larger switches (which are harder to drive and produce more charge-feedthrough). Of particular importance in this work, larger pump currents will also require large capacitors in the loop-filter to absorb the charge.

\section{C.3.1 An Aside: UP/DN Mismatch and Compliance Range}

There is an abundance of literature which emphasizes close matching of UP/DN current sources across the compliance range of the charge-pump. To achieve highimpedance current sources, cascode arrangements are often used to keep UP/DN current sources matched across a wider range. Reasons cited for the matching are to minimize: 1) steady-state phase offset, 2) CP on-time (and thus noise), and 3) reference spurs.

Assume, for the moment, a 1\% UP/DN mismatch, which is often cited on specification sheets as the end of the compliance region, and a $500 \mathrm{ps}$ dead-zone avoidance pulse. This would result in $5 p s$ steady state offset (typically an insignificant number), and the UP/DN pumps would be on for 505ps/500ps instead of 500ps/500ps for an increased pump noise of $0.09 d B$ (also insignificant). Finally, the extra $5 p s$ creates a sawtooth waveform at the comparison frequency. In the pessimistic case of a $10 \mathrm{GHz}$ $\mathrm{VCO}$, the total power in this sawtooth is $-26 \mathrm{dBc}$, but occurs at multiples of the reference frequency and is spread from $f_{\text {ref }}$ to $1 /\left(5 p s * f_{\text {ref }}\right)$ before the first null. For a $50 \mathrm{M} \mathrm{Hz}$ reference, this power is distributed across $>4 k$ tones, with each $\approx-62 d B c$ before filtering. Since the comparison frequency is at least 10x the loop-BW (typically more), and $3^{\text {rd }}$ order filters are common, this would be attenuated by another $60 d B$ and appear at $-122 d B c$ at the reference offset. Even in this pessimistic case, this is insignificant compared to typical reference spur specifications which call for between $-60 \mathrm{dBc}$ and $-100 \mathrm{dBc}$. Under these assumptions, a $10 \%$ mismatch results in a reference spur of $-102 \mathrm{dBc} / \mathrm{Hz}$ which is still a very respectible number. 
In practice, independent measurements show that despite current sources matched to better than $1 \%$ (in DC simulations), current sources may require an actual mismatch of over $50 \%$ (at high comparison frequencies) to eliminate the reference spur, further indicating that DC matching of current sources is a poor choice when considering the increased complexity. The author's conclusion is that achieving UP/DN current mismatch of $1 \%$ is a wasted effort.

\section{C.4 Charge Pump Current Sources}

Given the preceding discussion, it is suggested that the designer fight the temptation to create superbly matched and cascoded current sources, and in the process gains can be achieved in terms of area, complexity, and parasitic reduction.

Start with ideal UP/DN signals driving ideal switches, but real current sources/sinks. Driving the UP/DN signals with pulses of width 550ps/500ps will approximate lock conditions for the purpose of noise simulations. Start with a mirror ratio of 1:1 from the reference side, and worry about reducing wasted reference-path current later.

You may quickly realize that the current sources do not like to turn on/off quickly. The problem is that while the charge-pump switch is off, the current source/sink charges its drain to the rail (either VDD or VSS), and so $V_{D S}=0$ and the transistor is cut-off. It takes some time after the switch closes again for $V_{D S}$ to stabilize and for the current to reach its expected value. (This time depends on the size of the parasitic cap on the drain of the current sources/switches, and on the conductance of the CP switch). Also during this time, there is charge delivered to the load, but its the uncontrolled excess of $V D D-V_{c}$ that was stored on the parasitic capacitances. A typical approach is to introduce a dummy branch into the charge-pump so that the current is always flowing and $V_{D S}$ 's are always high enough to keep the transistors saturated. Various levels of complexity exists for these dummy branches - from complete duplicates of the mission-mode paths to simple switches to VDD/2 bias lines. For the moment, the interest is in characterizing the noise inherent in the charge-pump current sources themselves, and not in the auxiliary circuits. To keep the current sources sane without getting into unnecessary (at the moment) complexity, one can add ideal switches (with complemented inputs) to a dummy path, and 
an ideal voltage-controlled-voltage-source (aka op-amp) to drive the dummy node to match the mission-mode output node.

With the same setup as the PFD testing (a PSS/pnoise simulation, driving into a voltage source, and applying the same scaling), the noise contribution of the current source can be simulated. As the current-source transistor gets larger $\left(\mathrm{W}^{*} \mathrm{~L}\right)$, the flicker noise falls. As current goes up, noise goes up with $\sqrt{I_{D S}}$, but output referred noise actually goes down because the signal strength grows linearly. Start from a low-current/hi-noise scenario and increase current levels and $\mathrm{W} / \mathrm{L}$, keeping $V_{g s} \approx V_{t h}+0.2$ (for a $V_{\text {eff }}=0.2$ ) until meeting the close in noise specifications with a few $\mathrm{dB}$ of margin to account for addition of the CP switches and PFD.

At this point, substitute the designed PFD for the ideal PFD and verify little or no depredation in total output noise (since the PFD should be about 7-10dB below the $\mathrm{CP}$ ).

\section{C.5 Charge Pump Switches}

At this point, the required charge-pump current is more-or-less defined. The chargepump switches should be able to switch this current to the load and reach steady-state within the dead-zone pulse width of the PFD. The faster the switch performs, the shorter the pulses from the PFD need to be. Keeping these pulses short keeps the pump off (and not contributing to noise) longer. This would argue for large switches, but the problem is, the larger switches have more parasitic capacitance (leading to charge-feedthrough and reference spurs) and are difficult to drive from the phasedetector (degrading both noise and power consumption). Also keep in mind that for each switch on the mission-mode side, another complementary switch is likely required on the dummy branch.

It is common to use either dummy transistors and/or transmission gates on the charge-pump switches to minimize charge-feedthrough effects, but they come at the cost of increased area, power consumption and parasitic capacitance.

One approach is to focus on the noise implications of these transistors first, and then tackle the transient feedthrough problems. Using the PFD and semi-ideal charge-pump from the last section, increase the dead-zone width such that the UP/DN pulses are on for longer durations, and the limited switching speeds should not be 
a problem (eg. 5050ps/5000ps) and resimulate the noise performance. It should be degraded by about $20 d B$ because the pump is on $10 \mathrm{x}$ longer.

Add ideal buffers between the PFD and CP switches, and replace the ideal switches with minimally sized transistors. Check the noise depredation. Sizing up the switch transistors will bring it closer to the ideal number, with diminishing returns. Once within $1-2 d B$ or it becomes clear that further increases are ineffective turn your attention to the PFD buffer string. Size the buffer string from the PFD such that the $\mathrm{W} / \mathrm{L}$ ratio of each stage is about $3 \mathrm{x}$ the previous stage. Use as many stages as necessary until the final drive $\mathrm{W}^{*} \mathrm{~L}$ is approx $1 / 3^{\text {rd }}$ the $\mathrm{W}^{*} \mathrm{~L}$ of the loading gate.

Resimulate the noise now that the ideal buffer is replaced with the buffer string. If there is a significant depredation $(>1 \mathrm{~dB})$, return to the section on the PFD and optimize with a more realistic load.

Bring the mutual pulse width back down to $\approx 550 \mathrm{ps} / 500 \mathrm{ps}$ and resimulate with both ideal and real switches to check the noise depredation. Switch to a transient simulation and verify that the pump current reaches steady-state over the dead-zone pulse. If it does not, increase switch size further, or increase the dead-zone width of the PFD (by increasing the delay in the reset path).

\section{C.6 The Loop Filter}

With the charge-pump and VCO roughly designed, the next degree of flexibility is the loop bandwidth.

If fast lock-time is a priority, then the loop BW is normally set relatively wide. This helps eliminate VCO contributions, but makes the pump contribution significant out to further offsets. The lock process can be divided into two sections: 1) pull-in: which is the time it takes the VCO frequency to initially reach the target frequency, and 2) phase-stabilization: the time it takes to pull the VCO phase to within a certain number of degrees (often $5^{\circ}$ ) of steady state phase. The first stage is a non-linear process that depends on the hop distance, loop gain, cycle slipping, and a number of other factors. It can be sped-up and nearly eliminated by a variety of techniques. The second stage requires fine-grain stabilization of frequency and phase and typically takes about $5-10 / B W$. 
If the loop-BW is not constrained by lock-time, it will typically be chosen to reduce total noise while still meeting the phase-noise mask. This is done by setting it at the intersection of the open-loop VCO noise with the open-loop synthesizer noise (which is dominated by the charge-pump) as shown in Figure 2.8 .

With the loop-BW now set, the filter must be implemented. The main design variable on the $\mathrm{CP}$ was current. In order to meet tight noise constraints, pump current needs to be increased. If using a conventional single-voltage VCO, the gain of the $\operatorname{VCO}\left(K_{V}\right)$ is also fixed in order to satisfy application requirements (frequency-range) across expected PVT fluctuations. Given a fixed loop-gain $K_{V} * K_{C P}$, loop-BW $B W$, multiplication ratio and phase margin, the loop components are essentially fixed. A set of example parameters, used in this work, calls for $K_{V}=148.5 \mathrm{MHz} / \mathrm{V}, I_{C P}=$ $15 u A, B W=200 \mathrm{kHz}, P M=50^{\circ}, M=8$, and would lead to $C_{1}=420 p F, R_{1}=$ $5.2 \mathrm{kOhm}, \mathrm{C}_{2}=64 \mathrm{pF}$. In $0.18 \mathrm{um}$ TSMC CMOS, a capacitance of $484 \mathrm{pF}$ would take $\approx 420 \mathrm{kum}^{2}\left(1 \mathrm{fF} / \mathrm{um}^{2}\right.$ TSMC $\left.0.18 \mathrm{um} \mathrm{MiM} \mathrm{cap}\right)$, or $54 \mathrm{x}$ the size of the circuit presented in this work.

If using the cascaded pump structure of this work, the control range of the VCO is partitioned into sections and the capacitance requirements can be reduced. Furthermore, because the individual capacitances are much smaller, more area effcient MOSCAPs $\left(2.3 \mathrm{fF} / \mathrm{um}^{2}\right)$ can be used without suffering from the higher dielectric leakage effects.

The active-area requirements of the cascaded charge-pump and filter are 2.6 gates $\left(31.72 \mathrm{um}^{2}\right) /$ stage. Though the circuit highlighted in this work rotates 3 shared filter stages around the circuit, 5 stages should be shared for cases where a large number of stages are used and $R_{1}$ is therefore high. The total area is roughly:

$$
\text { area }=\text { ActArea }_{\text {perstg }} * N+5 * C_{\text {total }} /\left(\text { Area }_{\text {perunitcap }} * N\right)
$$

This yields an optimal number of charge-pump stages of:

$$
N_{\text {opt }}=\sqrt{\frac{5 * C_{\text {total }}}{\text { Area }_{\text {perunitcap }} * \text { ActArea } a_{\text {perstg }}}}
$$




\section{C.7 Summary}

A procedure has been suggested that allows a PLL designer to generate an efficient design that meets a phase noise mask with minimal iteration, area and power consumption. In summary, outside the loop-BW the limitation is the VCO whereas inside the loop-BW it should be the charge-pump current sources. If using the cascadedcharge pump, significant savings can be achieved by reducing the effective VCO gain and increasing the charge-pump gain without the requisite increase in filter sizes. 


\section{Appendix D}

\section{Characterizing Jitter}

\section{D.1 The Ambiguity of Jitter}

Unfortunately, an inappropriate and confusing lexicon has developed around the term jitter. Many authors, specifications and EDA tools will often use the same terms to mean very different things. Figure D.1, shows a sampling of the variety one encounters.

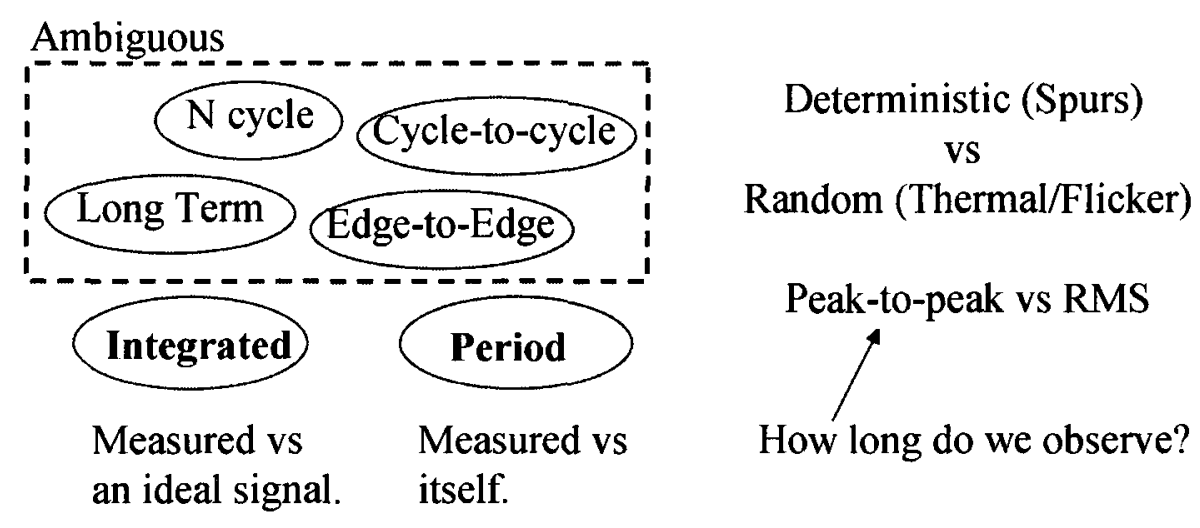

Figure D.1: The inappropriate lexicon of Jitter: A variety of terms used to describe jitter are ambiguous. There are two fundamental flavors of jitter depending on whether the measurement is referenced to itself (period jitter), or an ideal signal (integrated jitter). Further, jitter can be either deterministic (caused by periodic interference) or random (typically caused by noise).

There are fundamentally two types of jitter, depending on whether the measurement reference is the signal itself (period jitter), or a fictitious ideal oscillator 
(integrated jitter). Often, but not universally, authors will use the terms cycle-tocycle, edge-to-edge, and period jitter to mean the same thing, while long-term jitter may be used synonymously with integrated jitter. Once again, though, there is no universally accepted standard and many confuse the two types unintentionally. Be wary and always look at the context of the discussion to determine which type of jitter is being discussed.

\section{D.1.1 Period Jitter}

Period jitter, Figure D.2, measures each output cycle as an independent entity, triggering off the first edge and measuring the time to the second edge. This is the measurement of interest for clocking digital circuits, where there is no long-term history of interest. It is also the type of jitter that is almost universally measured with a high-frequency time-domain sampling scope.

Period jitter - Measure each period independently.

No Phase noise equivalent.

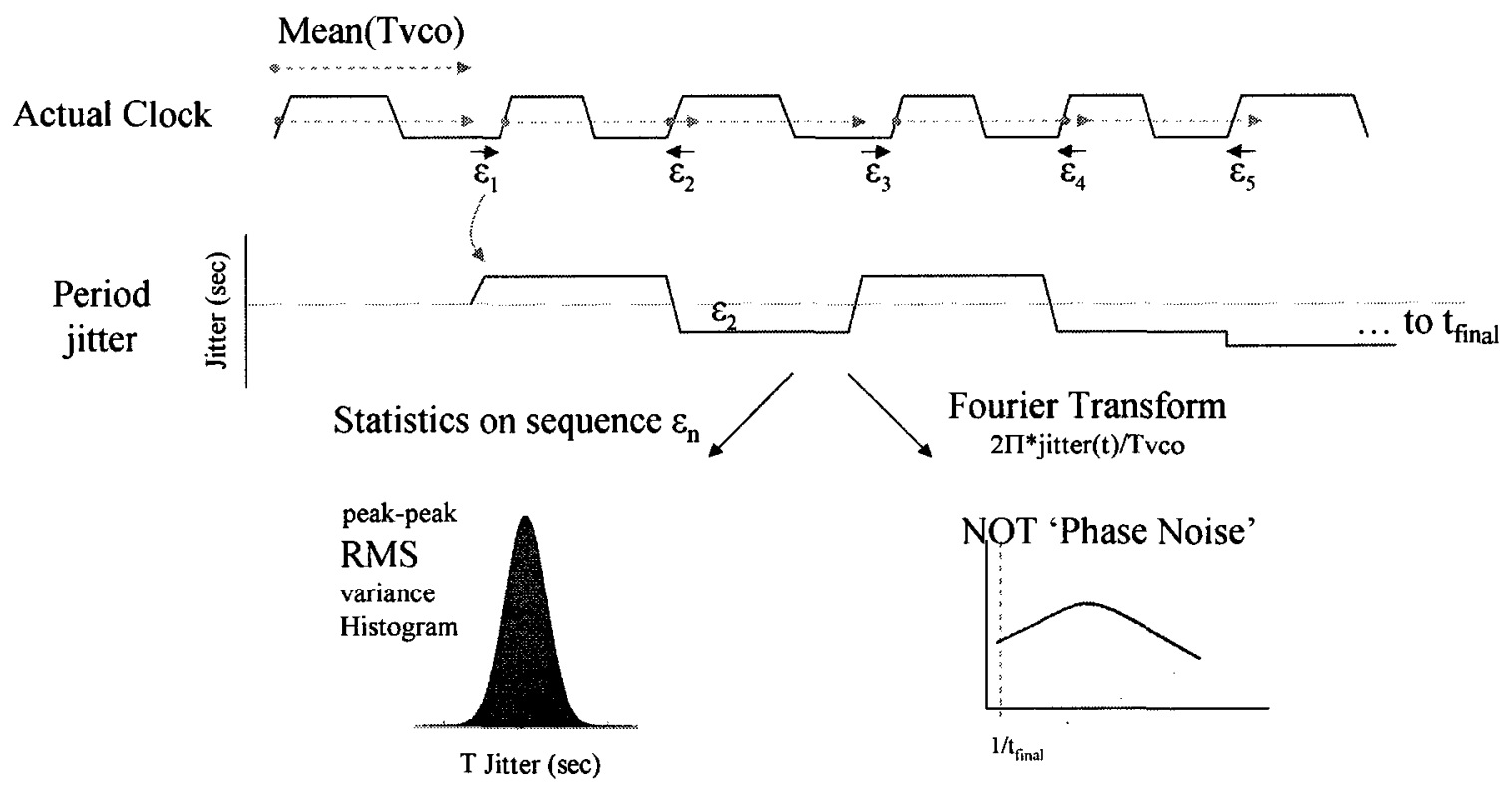

Figure D.2: Period Jitter: Each cycle is measured as an independent entity and compared against the average measurement. While the FFT of the error versus time can be done, this is NOT what is classically referred to as 'phase-noise'. 


\section{D.1.2 Integrated Jitter}

Integrated jitter, Figure D.3, measures the output against an ideal oscillator running independently from time $0 .{ }^{1}$ At any interesting phase event - eg. an edge crossing in a square wave - the error in time between the actual signal and the ideal one is recorded. With elegant simplicity, which the author has never seen presented elsewhere, the phase noise spectrum is simply the Fourier transform of this time domain jitter. ${ }^{2}$

Integrated jitter - compare each edge versus an ideal clock running independently.

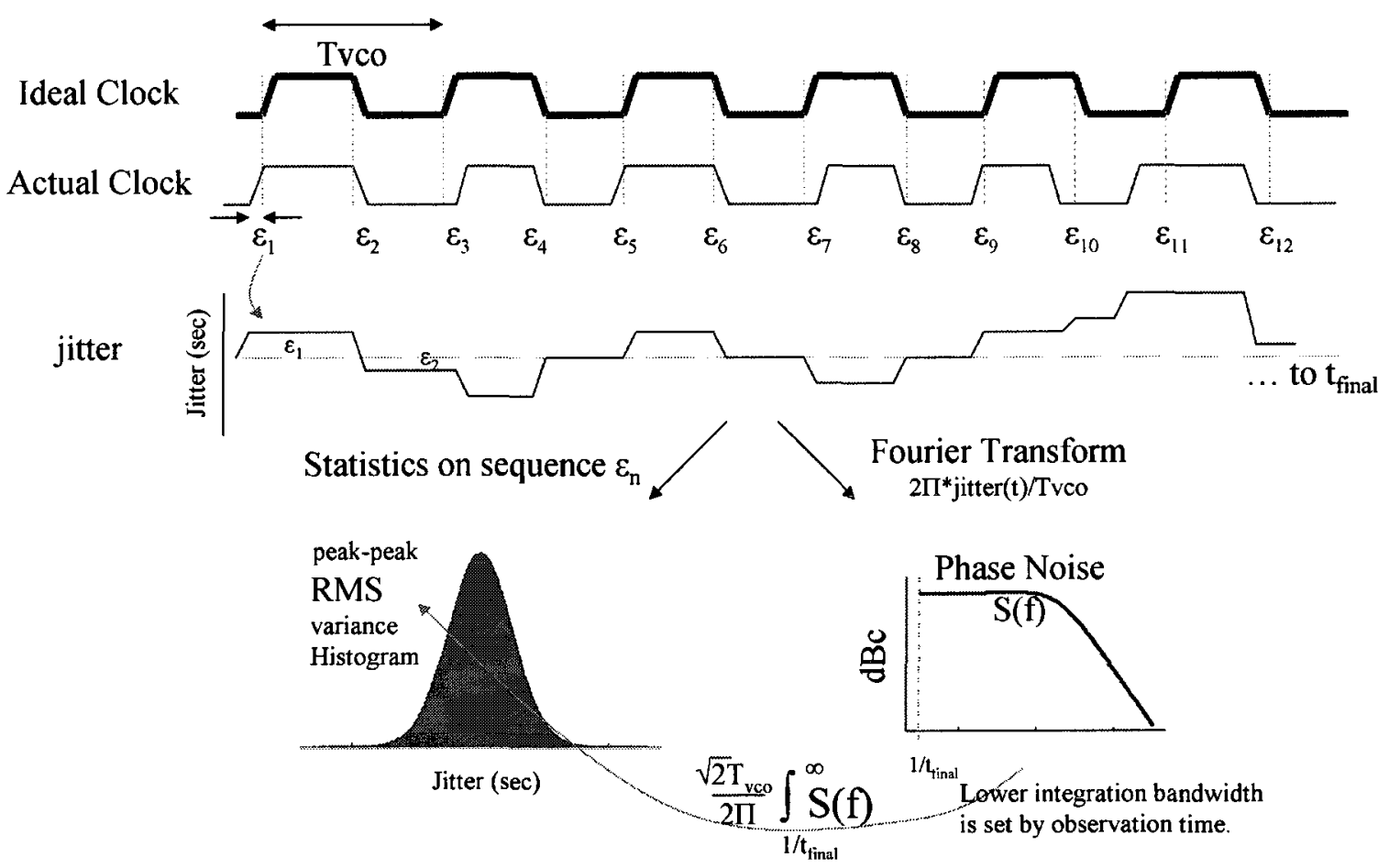

Figure D.3: Integrated Jitter : Phase noise is simply the Fourier transform of the integrated jitter vs time.

It is rare to see time-domain measurements of integrated jitter. Instead, the RMS jitter tends to be calculated by integrating the phase noise spectrum.

\footnotetext{
${ }^{1}$ In practice it is difficult to create an ideal oscillator.

${ }^{2}$ To scale appropriately to dBc, the jitter-vs-time should be scaled by $20 * \log 10\left(j i t t e r(t) \frac{2 \pi}{T_{V C O}}\right)$.
} 


\section{Integration Limits/Observation Time}

One difficulty with converting from phase-noise to an equivalent integrated jitter power is deciding on the integration limits of the phase-noise spectrum. Choice of the integration limits typically depends on the system where the synthesizer is used. For example, in packet based communications systems, the oscillator drift/variation is of interest only for the duration of the packet. Any lower frequency fluctuations are of little consequence. Choosing a lower integration limit of $\approx 0.1 / t_{\text {packet }}$ would be a reasonable boundary. To chose the upper boundary, the oscillator will typically go through some band-limiting components or into a band-limited communication system. This information should be used to estimate an upper integration limit.

\section{D.1.3 Linking Period Jitter and Phase Noise}

Since period based measurements are important in SERDES and clocking applications, it is useful to determine the link between them and the phase-noise spectrum (or integrated jitter performance) of the base synthesizer. The system level simulator described in Chapter 3 was used to characterize the difference between the two cases, and the results are discussed in Figure D.4.

Of particular relevance, the period based measurement provides a significant advantage by suppressing the phase noise by $20 \mathrm{~dB} / \mathrm{dec}$ coming in from a corner frequency of $f_{V C O} / 8$. Ironically, for higher frequency VCOs it becomes easier to achieve lower period jitter (in terms of seconds). 


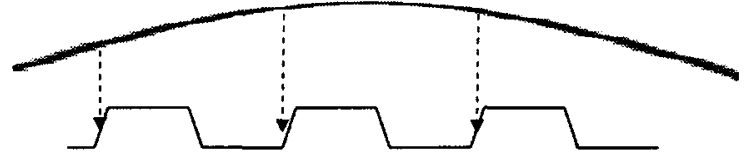

a) Low Frequency: Period jitter measurements reject low frequency noise/interference since the aggressor doesn't change much between independent cycles.

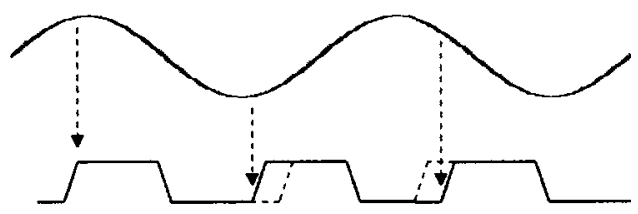

b) Noise/interference near half the VCO frequency is twice as damaging compared to measurement against an immovable reference.

c) Transfer function due to Period-by-period measurement.
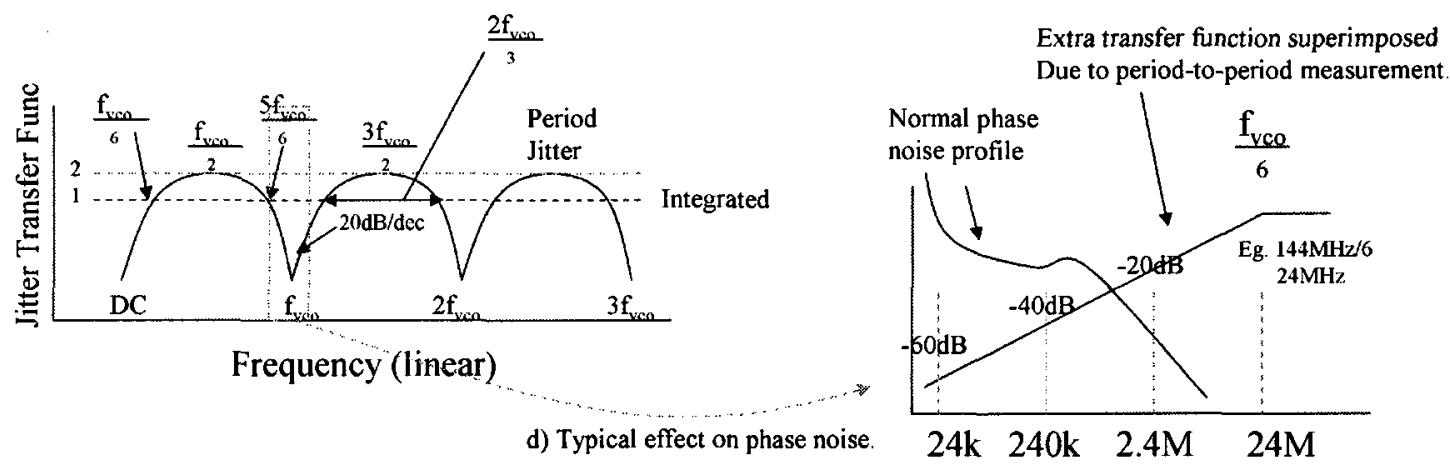

Figure D.4: Linking Period jitter to Phase Noise: a) Since a period jitter measurement occurs over a very short timescale, it is relatively insensitive to low frequency (or low offset frequency) noise or disturbances. b) If noise or interference is near half the frequency of the VCO, a period measurement will emphasize it by $2 \mathrm{x}$ compared to a measurement against an ideal source, since both the reference and desired measurement edge can move due to noise. c) The high-pass response of the period jitter measurement creates notches at $f_{V C O}$ and its harmonics, whereas the susceptibility of both the reference edge and measurement edge to noise makes increases the noise by $6 \mathrm{~dB}$ at sub-harmonics. d) Since the notch occurs at the VCO frequency, where the phase-noise of the synthesizer is dominant, the high-pass characteristic suppresses the phase noise considerably. 


\section{References}

[1] Simon Tam, Stefan Rusu, Utpal Nagarji Desai, Robert Kim, and Ji Zhang, "Clock generation and distribution for the first ia-64 microprocessor," IEEE JSSC, vol. 35 , no. 10 , pp. $1545-1552$, Nov. 2000.

[2] T. Olsson and P. Nilsson, "An all-digital pll clock multiplier," in IEEE AsiaPacific Conf. on ASICs, 2002, pp. 275-278.

[3] C Fernando, K Maggio, R Staszewski, and J T Jung, "All-digital tx frequency synthesizer and discrete-time receiver for bluetooth radio in 130-nm cmos," IEEE JSSC, vol. 39, no. 12, pp. 2278-2291, Dec. 2004.

[4] Dean Banerjee, PLL Performance, Simulation, and Design, National Semiconductor, 1998.

[5] Byung-Guk Kim and Lee-Sup Kim, "A 250-mhz 2-ghz wide-range delay-locked loop," IEEE JSSC, vol. 40, no. 6, pp. 1310-1321, Jun. 2005.

[6] John G. Maneatis, "Low-jitter and process-independent dll and pll based on self-biased techniques," IEEE ISSCC, in Proceedings, p. 130, 1996.

[7] Hee-Tae Ahn and David J. Allstot, "A low-jitter 1.9-v cmos pll for ultrasparc microprocessor applications," IEEE JSSC, vol. 35, no. 3, pp. 450-454, 2000.

[8] R.B. Staszewski et al, "All-digital tx frequency synthesizer and discrete-time receiver for bluetooth radio in 130-nm cmos," IEEE JSSC, vol. 39, no. 12, pp. 2278-2291, Dec. 2004.

[9] B. Razavi, "Challenges in the design of high-speed cdr circuits," IEEE Communications Magazine, vol. 40, no. 8, pp. 94-101, Aug. 2002.

[10] W. F. Egan, Frequency Synthesis by Phase Lock, 2nd Edition, John Wiley \& Sons, 2000. 
[11] John Rogers Calvin Plett and Foster Dai, Integrated Circuit Design for HighSpeed Frequency Synthesis, Artech House, 2006.

[12] F. Gardner, "Charge-pump phase-lock loops," IEEE Trans. Communications, vol. 28 , no. 11, pp. 1849-1858, Nov. 1980.

[13] Dennis Fischette, "Practical phase-locked loop locked loop design," ISSCC Tutorial Session, 2004.

[14] Jong-Tae Kwak, Chang-Ki Kwon, Kwan-Weon Kim, Seong-Hoon Lee, and Joong-Sik Kih, "A low cost high performance register-controlled digital dll for 1 gbpsx32 ddr sdram," VLSI Circuits, 2003. Digest of Technical Papers. 2003 Symposium on, pp. 283- 284, 12-14 June 2003.

[15] Amr Fahim, "A compact, low-power low-jitter digital pll," European Solid-State Circuits. ESSCIRC'03. Conference, pp. 101-104, 2003.

[16] James C. Candy and Gabor C. Temes, "Oversampling methods for a/d and d/a conversion," IEEE Press, 'Oversampling Delta-Sigma Data Converter: Theory, Design, and Simulation', pp. 1-26, 1992.

[17] R. Jacob Baker Harry W. Li and David E. Boyce, CMOS Circuit Design, Layout, and Simulation., The Institute of Electrical and Electronics Engineers, 1998.

[18] Nicola Da Dalt and Christoph Sandner, "A subpicosecond jitter pll for clock generation in 0.12-um digital cmos," IEEE JSSC, vol. 38, no. 7, pp. 1275-1278, July 2003.

[19] Ramin Farjad-Rad, William Dally, Hiok-Tiaq Ng, Ramesh Senthinathan, M.J. Edward Lee, Rohit Rathi, and John Poulton, "A low-power multiplying dll for low-jitter multigigahertz clock generation in highly integrated digital chips," IEEE JSSC, vol. 37, no. 12, pp. 1804-1812, 2002.

[20] Kuo-Hsing Cheng, Shu-Ming Chung, Yu-Lung Lo, and Shu-Yu Jiang, "A 2.2 ghz programmable dll-based frequency multiplier for soc applications," IEEE AsiaPacific Conference on Advanced System Integrated Circuits 2004, pp. 72-75, Aug 2004. 
[21] Chulwoo Kim, In-Chul Hwang, and Sung-Mo (Steve) Kang, "A low-power smallarea 7.28-ps-jitter 1-ghz dll-based clock generator," IEEE JSSC, vol. 37, no. 11, pp. 1414-, 2002.

[22] Xintian Shi, K. Imfeld, S. Tanner, M. Ansorge, and P-A. Farine, "A low-jitter and low-power cmos pll for clock multiplication," IEEE ESSCIRC, pp. 174 $177,2006$.

[23] Akihide Sai, Daisuke Kurose, Takafumi Yamaji, and Tetsuro Itakura, "A lowpower low-noise clock signal generator for next-generation mobilewireless terminals," IEICE Trans. on Fundamentals of Electronics, Communications and Computer Sciences, vol. E91A, no. 2, pp. 557-560, 2008.

[24] Ching-Che Chung and Chen-Yi Lee, "An all-digital phase-locked loop for highspeed clock generation," IEEE JSSC, vol. 38, no. 2, pp. 347-351, 2003.

[25] Jae Joon Kim et al, "A low-jitter mixed-mode dll for high-speed dram applications," IEEE JSSC, vol. 35, no. 10, pp. 1430-1436, 2000.

[26] A. Maxim, "A 0.16-2.55-ghz cmos active clock deskewing pll using analog phase interpolation," IEEE JSSC, vol. 40, no. 1, pp. 110-131, Jan. 2005.

[27] Seung-Jun Bae, Hyung-Joon Chi, Young-Soo Sohn, and Hong-June Park, "A vcdl-based $60760-\mathrm{mhz}$ dual-loop dll with infinite phase-shift capability and adaptive-bandwidth scheme," IEEE JSSC, vol. 40, no. 5, pp. 1119-1129, 2005.

[28] R.B. Staszewski et al, "A first multigigahertz digitally controlled oscillator for wireless applications," IEEE Trans. on Microwave Theory, vol. 51, no. 11, pp. 2154-2164, Nov. 2003.

[29] M. Thamsirianunt and T. Kwasniewski, "Cmos vcos for pll frequency synthesis in ghz digital mobile radio communications," IEEE JSSC, vol. 32 , no. 10, pp. 1511-1524, 1997.

[30] Kuo-Hsing Cheng, Yu-Chang Tsai, Kai-Wei Hong, and Yen-Hsueh Wu, "A low jitter self-calibration pll for 10gbps soc transmission links application," in IEEE International Conference on Electronics, Circuits and Systems, 2008, pp. 786789 . 
[31] Stefano Zanella, Alessandra Nardi, Michele Quarantelli, Andrea Neviani, and Carlo Guardianit, "Analysis of the impact of intra-die variance on clock skew," Statistical Metrology, 1999. IWSM. 1999 4th International Workshop on, pp. 14-17, 1999.

[32] Eby G. Friedman, Clock Distribution Networks in VLSI Circuits and Systems, IEEE Press, Piscataway, NJ, 1995.

[33] A.V.Mule, E.N.Glytsis, T.K.Gaylord, and J.D.Meindl, "Electrical and optical clock distribution networks for gigascale microprocessors," IEEE Trans. VLSI, vol. 10 , no. 5, pp. 582-594, Oct 2002.

[34] D. M. Shapiro, Globally Asynchronous, Locally Synchronous Systems, PhD thesis, Stanford University, 1984. 